This thesis was accepted by the Department of Physics and Astronomy, The University of New Mexico, Albuquerque, New Mexico, in partial fulfillment of the requirements for the degree of Doctor of Philosophy in Optical Science. It is the independent work of the author and has not been edited by the IS-11 Writing and Editing staff.

An Affirmative Action'Equal Opportunity Employer

This report was prepared as an account of work sponsored by an agency of the

United States Government. Neither The Regents of the Unizersity of California, the United States Government nor any agency thereof, nor any of their employees, makes any warranty, express or implied, or assumes any legal liability or responsibility for the accuracy. completeness, or usefulness of any information, apparatus, preduct, or process disclosed, or represents that its uSe wnuld not infringe prizately owned rights. Reference herein to any specific commercial product, process, or service by trade name, frademark, mamufacturer, or otheravis', doss not necessarily constitute or imply its endorsement, recommendation, or facoring by The Rezents of the University of California, the United Siates Gonernment, or any agency thereof. The rien's and opinions of authors expressed herein do not necessarily state or reflect those' of The Reje'nts of the University of California, the United State's Government, or any agency therevef. 
Measurement of Intensity-Dependent Rates

of Above-Threshold Ionization (ATI)

of Atomic Hydrogen at $248 \mathrm{~nm}$

Thomas David Nichols* 


\section{Acknowledgments}

It is my pleasure to acknowledge many people who have contribuied to the success of this experiment. I am most indebted to my advisor at Los Aiamos National Lab, Dr. George Kyrala. George supervised my day to day work and provided most of the ideas that worked in this experiment. I am also indebted to my advisor at the University of New Mexico, Prof. Howard Bryant. I might not have finished at all without his encouragement. I would certainly not have finished my part of the project in two years if it had not already been started by George, Dr. Don Casperson, Dr. Larry Jones, and Dr. Gottfried Schappert of Group P-1 at LANL. I thank them also for their help and advice while I was there. I thank George and Don in particular for sharing their calculations of electron trajectories in the time-of-flight spectrometer. The P-1 technicians, Mike Maestas, Kurt Stetler, and Jan Studebaker, saved me countless hours in the lab and taught me many valuable st 'ls such as how to keep an ultrahigh vacuum system clean and how to choose a capacitor for a short-pulse circuit. In this dissertation, I have tried to distinguish between things I can claim credit for individually ("I measured ...") and group efforts ("We measured ..."). I depended on Group CLS-5, especially Dr. Toni Taylor, Sue Harper, and Jeff Roberts, to maintain and operate the LABS-I laser. They also provided the measurement of the pulse length. They went out of their way several times to help me when I needed a little extra laser time at the end of the day. Space does not permit listing all the others at LANL who helped in particular situations, discussed my work and theirs, or kept the paperwork flowing so that I could work in the lab, but they are a big part of the reason that Los Alamos is a good place to work. At UNM, Dr. Willielm Becker took the time on many occasions to help me understand the theory of ATI and to describe it accurately in my 
dissertation. I also thank Dr. Thomas Niemczyk of the Chemistry Department for serving on my committee. Finally, I thank Dr. Shih-I Chu for allowing me to use his theoretical results prior to publication, and Dr. George Csanak for correcting the misprint in the paper by Keldysh.

IBM and PC-AT are registered trademarks of International Business Machines Corporation. Microsoft is a registered trademark of Microsoft Corporation. Pyrex is a registered trademark of Corning Glass Works. 


\section{TABLE OF CONTENTS}

PAGE

Chapter 1. Introduction . . . . . . . . . . . . . . . . . . . . 1

1.1 Above Threshold Ionization . . . . . . . . . . . . . . . . 4

1.2 Experiments in ATI . . . . . . . . . . . . . . . . . . . . 5

1.3 Comparisons to ATI Theory . . . . . . . . . . . . . . . . 7

1.3.1 Selection of target gas . . . . . . . . . . . . . . . 8

1.3.2 Resonances . . . . . . . . . . . . . . . . . . . . . . 9

1.3.3 Electron counts . . . . . . . . . . . . . . . . . . . 11

1.4 Questions to be answered by this work . . . . . . . . 13

Chapter 2. Theory . . . . . . . . . . . . . . . . . . . . . . . 15

2.1 Strong Field Physics Before ATI . . . . . . . . . . . . . . 15

2.1.1 Perturbation Theory . . . . . . . . . . . . . . . 15

2.1.2 Ponderomotive Forces . . . . . . . . . . . 18

2.2 ATI Theory . . . . . . . . . . . . . . . . 20

2.2.1 Introduction . . . . . . . . . . . . . . . . . 20

2.2.2 Higher order perturbation theory . . . . . . . . . 21

2.2.3 Numerical integration of the Schrödinger equation . . . . 22

2.2.4 Keldysh-Faisal-Reiss (KFR) models . . . . . . . . . . . 22

2.2.5 Floquet calculations . . . . . . . . . . . . . . 25

2.2.6 Other methods . . . . . . . . . . . . . . . 26

2.3 Predictions from the theories . . . . . . . . . . . . 27

Chapter 3. Description of the experiment . . . . . . . . . . . 29

3.1 Overview . . . . . . . . . . . . . . . . . 29

3.2 Apparatus . . . . . . . . . . . . . . . 30

3.2 .1 Vacuum system . . . . . . . . . . . . . . . . . . . 30

3.2.2 Atomic hydrogen source . . . . . . . . . . . . . . . 34

3.2.3 Optical system . . . . . . . . . . . . . . . . . . . 36

3.2.3.1 LABS-I laser . . . . . . . . . . . . . . . . . . . 36

3.2.3.2 Windows, mirrors, and beamsplitters . . . . . . . . 37

3.2.3.3 Photodiode and joulemeter . . . . . . . . . . . 38

3.2.3.4 Mechanical Support . . . . . . . . . . . . . . . . 39

3.2.4 Electron time-of-flight spectrometer . . . . . . . . . . . 39

3.2.4.1 Theory of operation . . . . . . . . . . . . . . . . 40

3.2.4.2 Construction . . . . . . . . . . . . . . . . 42

3.2.5 Signal processing and data storage system . . . . . . . 44

3.2.5.1 Microchannel plate electron detector . . . . . . . . 44

3.2.5.2 CAMAC and GPIB devices . . . . . . . . . . . . . 46

3.2.5.3 Data collection programs . . . . . . . . . . . . . . 48 
3.2.6 Alignment of the interacting beams . . . . . . . . . . . 52

3.3 Calibration . . . . . . . . . . . . . . . . . . . . . . 54

3.3.1 Irradiance at focus . . . . . . . . . . . . . . . . . . . 54

3.3.2 Density of atomic hydrogen . . . . . . . . . . . . . . 57

3.3.3 Number of electrons produced per unit signal recorded . . . 58

3.3.4 Time-of-flight spectrometer accuracy and resolution . . . . 60

3.4 Operation . . . . . . . . . . . . . . . . . . . . . . . . 61

3.5 Additional considerations . . . . . . . . . . . . . . . . . 64

3.5.1 Background gases . . . . . . . . . . . . . . . . . . . 64

3.5.2 Limitations on temperatures for baking . . . . . . . . . 64

3.5.3 Transients in RGA readings . . . . . . . . . . . . 65

3.5.4 Pulse stretching by the windows . . . . . . . . . . 68

Chapter 4. Data, analysis, and conclusions . . . . . . . . . . . 69

4.1 Features of the electron spectra . . . . . . . . . . . . . . . 69

4.2 Effect of different values of peak irradiance . . . . . . . . . . 71

4.3 Isolation of atomic hydrogen signal from other signals . . . . . 75

4.4 Space Charge . . . . . . . . . . . . . . . . . . . . . . . 79

4.5 Ponderomotive shifts . . . . . . . . . . . . . . . . . . 81

4.6 Comparison of measurements to theoretical predictions . . . . . 83

4.7 Conclusions . . . . . . . . . . . . . . . . . . . . . . . 90

4.8 Suggestions for further work . . . . . . . . . . . . . . 94

Appendix A. Units, magnitudes, and dimensionless parameters 95

Appendix B. Calculations concerning the vacuum system . . . 99

1. Sensitivity of the RGA to atomic and molecular hydrogen . . . . 99

2. Calculations of flow rates and recombination rate . . . . . 102

Appendix C. Measurement of focal volume characteristics . . 105

1. Experimental setup . . . . . . . . . . . . . . 105

2. Procedure . . . . . . . . . . . . . . . . . 106

3. The $M^{2}$ model . . . . . . . . . . . . . . . . . . . . . 107

4. Analysis methods . . . . . . . . . . . . . . . . . . . . 109

5. Results . . . . . . . . . . . . . . . . . . . . . 111

5.1 Waist radius from single-shot measurements . . . . . . 111

$5.2 Z_{R}$ and $M^{2}$ from single-shot measurements . . . . . . 113

5.3 Average waist radius from averaged readings . . . . . . . 117

6. Discussion . . . . . . . . . . . . . . . . 119

Appendix D. Microchannel plate (MCP) electron detector . . 123

1. Description . . . . . . . . . . . . . . . . 123

2. Measurement of system gain . . . . . . . . . . . . 124

3. Measurement of subsystem gains . . . . . . . . . . . . . . 128

4. Comparison to manufacturer's specifications . . . . . . . . . 130

5. Additional observations . . . . . . . . . . . . . . . 132 
Appendix E. Listings of computer programs . . . . . . . . . 134

1. RUN . . . . . . . . . . . . . . . . . . . . . . . . . . 134

2. NEWRUN . . . . . . . . . . . . . . . . . . . . . 138

3. CONVERT1 . . . . . . . . . . . . . . . . . . . . 153

4. ERUN . . . . . . . . . . . . . . . . . . . 155

5. DRUN . . . . . . . . . . . . . . . . . . . . . 173

6. DISCRIM . . . . . . . . . . . . . . . . . . 179

References ................... 182 


\section{LIST OF FIGURES}

FIGURE

PAGE

1. Simplified energy level diagram of a hydrogen atom showing electron peaks from absorption of three, four, and five $5 \mathrm{eV} \mathrm{KrF}$ photons . 5

2. Three-photon ionization rates from the hydrogenic state $1 \mathrm{~s}$. . 10

3. Shifting and broadening of atomic hydrogen energy levels by a strong $\mathrm{KrF}$ laser field . . . . . . . . . . . . . . . . . . . . . . . 11

4. Predictions from five models of MPI rates for atomic hydrogen and linearly polarized $248 \mathrm{~nm}$ light . . . . . . . . . . . . . . . . . . . . . 28

5. Schematic diagram of the experimental apparatus . . . . . . . 32

6. Schematic view of the interaction region . . . . . . . . . . 33

7. Block diagram of CAMAC and GPIB devices . . . . . . . . . . 47

8. Height of the $S_{0}$ peak of atomic hydrogen as the focusing mirror is translated through the point of maximum signal . . . . . . . 55

9. Partial pressures of (a) Mass-2 $\left(\mathrm{H}_{2}\right)$ and (b) Mass-1 (H) as the discharge was flashed on for $150 \mathrm{msec}$ every $400 \mathrm{msec}$. . . . . . . 66

10. Electron spectra, recorded with a $4 \mathrm{~V}$ retarding potential, vs. time of flight

11. Electron spectra, recorded with a $4 \mathrm{~V}$ retarding potential, vs. electron energy .

12. Electron spectra collected at different irradiances . . . . . . . 73

13. Four electron spectra showing a change in number of electrons produced with no change in pulse energy

14. Electron time-of-flight spectrum of molecular hydrogen at an irradiance of $6 \times 10^{13} \mathrm{~W} / \mathrm{cm}^{2}$

15. Comparison of measured and predicted total numbers of electrons produced in the interaction region by MPI of atomic hydrogen, using the smallest measured waist radius $W_{0}=35 \mu \mathrm{m}$. . . . . . . . .

16. Data of Fig. 15 cornpared to areas of the $S_{1}$ and $S_{2}$ ATI peaks in the same electron spectra

17. Comparison of measured and predicted total numbers of electrons produced in the interaction region by MPI of atomic hydrogen, using the largest measured waist radius $W_{0}=45 \mu \mathrm{m}$. 
18. Highest values of transmission from vertical scan through focus . . 112

19. Highest values of transmission from axial scan through focus . . . 114

20. Highest values of transmission from transverse scan $3.3 \mathrm{~mm}$ from best focus . . . . . . . . . . . . . . . . . 118

21. Highest values of transmission from two horizontal scans through focus $\mathbf{1 1 9}$

22. Average values of transmission from the same scans as in Fig. 21, compared to model with $W_{0}=35 \mu \mathrm{m}$. . . . . . . . . . . . . 120

23. Average values of transmission from the same scans as in Fig. 21, compared to model with $W_{0}=45 \mu \mathrm{m}$. . . . . . . . . . . . . 121

24. Average values of transmission from vertical scans of $20 \mathrm{Apr} 90 \mathrm{com}$ pared to model with $W_{0}=35 \mu \mathrm{m}$. . . . . . . . . . . . . . . 122

25. Distributions of recorded signals from single electrons detected by the MCP at three applied voltages . . . . . . . . . . . . . . 126

26. Distribution of MCP pulse heights read from oscilloscope traces . . 129

27. Response of the transient digitizer to short pulses arriving at different times in the cycle of operation . . . . . . . . . . . . . . 130

28. Histogram of the response of the transient digitizer to short pulses arriving at different times in the cycle of operation . . . . . 131 


\section{LIST OF TABLES}

TABLE

PAGE

1. Comparison of generalized cross sections caiculated by LOPT for MPI of atomic hydrogen by linearly polarized $248 \mathrm{~nm}$ light . . . . . 27

2. Series of electron spectra collected $4 \mathrm{Jun} 90$ showing a change in number of electrons produced with no change in pulse energy

3. Widths (FWHM) of atomic hydrogen $S_{0}$ ATI peak at three irradiances and four atomic densities . . . . . . . . . . . . . . . 80

4. Irradiances calculated from ponderomotive shifts in data of $30 \operatorname{ct} 89$

5. Irradiances calculated from ponderomotive shifts in data of 12,21 , and $27 J u n 90$

6. Uncertainties in measured electron counts and irradiances, including the normalization to a specified gas density

7. Atomic units related to cgs and other commonly used units . . 95

8. Electron ionization cross sections at $60 \mathrm{eV}$. . . . . . . . . 102

9. Flow rate into the lower chamber on 12Jun90 . . . . . . . . . 103

10. Comparisons of calculated pressures including a model for recombination on the walls of the lower chamber . . . . . . . . . . . . . 104

11. Chi square per degree of freedom from three scans in the plane of best focus

12. Chi square per degree of freedom for an axial scan with nine degrees of freedom (two data points omitted) . . . . . . . . . . . 116

13. Chi square per degree of freedom for an axial scan with eleven degrees of freedom (all data points used)

14. Chi square per degree of freedom for an axial scan with eleven degrees of freedom, assuming $z_{0}=3.3 \mathrm{~mm}$

15. Potentials within MCP assembly for supply voltage of $2.209 \mathrm{kV}$. . 124

16. Gain of the electron detection system consisting of MCP detector and transient digitizer . . . . . . . . . . . . .

17. Calculated MCP gain and TR8818 output compared to measured TR8818 output 


\title{
MEASUREMENT OF INTENSITY-DEPENDENT RATES OF ABOVE-THRESHOLD IONIZATION (ATI) OF ATOMIC HYDROGEN AT $248 \mathrm{~nm}$
}

\section{by}

\section{Thomas David Nichols}

\begin{abstract}
Measured rates of multiphoton jonization (MPI) from the ground state of atomic hydrogen by a linearly polarized, subpicosecond $\mathrm{KrF}$ laser pulse at $248 \mathrm{~nm}$ wavelength are compared to predictions of lowestorder perturbation theory, Floquet theory, and Keldysh-Faisal-Reiss (KFR) theory with and without Coulomb correction for peak irradiances of $3 \times 10^{12} \mathrm{~W} / \mathrm{cm}^{2}$ to $2 \times 10^{14} \mathrm{~W} / \mathrm{cm}^{2}$. The Coulomb-corrected Keldysh model falls closest to the measured rates, the others being much higher (perturbation and Floquet) or much lower (KFR without Coulomb correction). At $5 \times 10^{13} \mathrm{~W} / \mathrm{cm}^{2}$, the number of ATI electrons decreased by a factor of approximately 40 with each additional photon absorbed. ATI of the molecular hydrogen background and of atoms from photodissociation of the molecules were also observed.

The experiment employed a crossed-beam technique at ultrahigh vacuum with an $\mathrm{rf}$-discharge atomic hydrogen source and a magneticbottle type electron time-of-flight spectrometer to count the electrons in the different ATI channels separately. The apparatus was calibrated to allow comparison of absolute as well as relative ionization rates to the theoretical predictions. This calibration involved measuring the distribution of irradiance in a focal volume that moved randomly and changed its size from time to time. A data collection system under computer control divided the time-of-flight spectra into bins according to the energy of each laser pulse. The irradiance calculated from the pulse energy, pulse length, and irradiance distribution agreed with measurements of the ponderomotive shift of the electron energies within the uncertainty of the shift measurements.

This is the first measurement of absolute rates of ATI in atomic hydrogen, and the first measurement of absolute rates of MPI in atomic hydrogen without a large factor to account for multiple modes in the laser field. As such, the results of this work are important to the development of ATI theories, which presently differ by orders of magnitude in their predictions of the ionization rates. They are also important to recent calculations of temperatures in laser-heated plasmas, many of which incorporate KFR theory. Since the KFR ionization rates are found to be much too low, these calculations may be considerably in error.
\end{abstract}




\section{Introduction}

The interaction of light with matter is one of the fundamental subjects of physics. Optical spectroscopy has been the primary tool for studying the structures of atoms and molecules since 1861, when the Fraunhofer lines in the solar spectrum were shown to result from atomic absorption. The observation of sharp lines in the absorption and emission spectra of gases led directly, if not immediately, to the quantum theory, and increasingly precise spectral measurements have contributed both qualitatively and quantitatively to our understanding of atomic and molecular processes to the present day.

For the first 100 years, however, the only way to do an optical experiment was to find something that emitted or absorbed light and describe the light. There was no way to design and control an optical experiment in the sense that electrical experiments, for example, were designed and controlled. A molecule to be studied had to be excited by a random process such as thermal agitation, electron bombardment, or absorption of broadband light. In certain cases, emission lines from mercury and other atoms could be used to excite the target molecule, but the choice of wavelengths was quite small, and the irradiances were quite low.

The situation chatiged dramatically with the invention of the laser. Today, the experimenter has a choice of wavelengths, power levels, pulse lengths, and degrees of coherence that makes many new kinds of experiments possible. The experiment described in this dissertation falls within the new area of nonlinear processes in strong fields. The term "strong" can be defined in several ways, but for this experiment it basically means irradiances greater than $10^{12} \mathrm{~W} / \mathrm{cm}^{2}$. (It is also important that the pulse reach high irradiances in less than a picosecond. If the rate of the process being studied becomes too high during the 
leading edge of the pulse, there will be no atoms left in their original state when the peak arrives.) Strong fields drive nonlinear processes that compete with or even overshadow those that had formerly been available for study. In addition, the classical ponderomotive force, too weak to observe without lasers, becomes a significant factor. As a result, it has become possible and necessary to develop theoretical models beyond the limits of conventional perturbation theory. Several methods are now used to include high-order interactions with the light field within a manageable number of calculations. These improved models, in turn, suggest new experiments. Strong-field physics is now an important area of research at laboratories and universities around the world.

One area of strong-field physics which has been extensively studied in recent years is multiphoton ionization (MPI). This is ionization of an atom or molecule by simultaneous absorption of several photons. The first order term of the perturbation expansion of the ionization rate in powers of irradiance does not provide a predicted rate for MPI, but higher order terms in the perturbation expansion do. An expansion which extends just to the first non-vanishing term for a multiphoton process is celled a "lowest order perturbation theory" (LOPT) for that process. The development of high-power lasers in the 1970's provided the means for testing LOP'T in MPI experiments. In these tests, the ionization rate could be measured by collecting either the ions or the electrons. When electrons were counted, however, there was the additional possibility of measuring their energies to see how many photons had been absorbed in the MPI process. When this was done, some atoms were found to have absorbed more than enough to supply their binding energy. This new phenomenon was called "above threshold ionization" (ATI). Some features of ATI could not be explained by LOPT, so an extensive series of experiments and theoretical advances ensued. Part of the interest in ATI is due to its effect on the way plasmas are heated by strong laser fields. 
This dissertation is based on measurements of a particularly simple case of MPI to which several theoretical approaches may be applied. The target was atomic hydrogen, for which exact wave functions are known in the zero-field limit. The photon energy of $5 \mathrm{eV}$ made the ionization process nonresonant, since two photons are not enough to reach the first excited state of hydrogen, while three are enough to ionize the atom. Electron signals were recorded in the form of time-of-flight spectra, so that the appearance of ATI could be observed. The peak irradiance was varied from $3 \times 10^{12} \mathrm{~W} / \mathrm{cm}^{2}$ to $2 \times 10^{14} \mathrm{~W} / \mathrm{cm}^{2}$ to cover the range from the appearance of MPI signals to depletion of the target atoms at the center of the focal region. Three ATI peaks coisld be distinguished at high irradiance. Measured total rates of electron production were less than those predicted by high order perturbation and Floquet models, but significantly higher than those calculated by the Reiss and Keldysh methods with Volkov final states. The measured rates fell nearest to the Coulomb-corrected Keldysh model, but they did not agree closely with this model, either.

This chapter continues with an introduction to the specific process being studied and the questions to be answered by this work. Chapter 2 summarizes the theory. Chapter 3 describes the experiment, detailing the construction, alignment, calibration, and operation of the apparatus. Chapter 4 presents representative data, demonstrating the effects of removing the atomic targets, varying the laser intensity, and introducing space charge by ionizing more atoms. Chapter 4 also describes analysis techniques used to isolate the signal of interest from the others present in the data, to find the point at which all atoms in the focal volume have been ionized, and to check internal consistency of the data. Finally, Chapter 4 presents conclusions from this work and suggestions for future experiments. Additional details related to the experiment appear in the appendices. 


\subsection{A bove Threshold Ionization}

Above threshold ionization, also known as "excess photon ionization", is a process that occurs only in strong electromagnetic fields. It differs from ordinary MPI in that the electron emerges with more than one unit kw of kinetic energy, having absorbed more photons from the electromagnetic field than the minimum number required to supply the binding energy of the atom or molecule. Figure 1 shows the relationship between the atomic energy levels, the photon energy, and the ATI electron spectrum. In the case shown, atomic hydrogen with $5 \mathrm{eV}$ photons, the electron cannot absorb one or two photons, because there is no resonant atomic level. Absorption of three photons frees the electron with $1.4 \mathrm{eV}$ of kinetic energy. The resulting peak in the electron energy spectrum is labeled $S_{0}$. Electrons belonging to the second $\left(S_{1}\right)$ peak have $6.4 \mathrm{eV}$ of kinetic energy, having absorbed one excess photon. Electrons in the $S_{2}$ peak have absorbed two excess photons, and so forth. The total number of photons absorbed may be written as $N_{0}+S$, where $N_{0}$ photons supply the zero-field binding energy $E_{B}$, and $S$ is the number of excess photons. Thus the $S_{2}$ peak of $H$ is described by $N_{0}=3$ and $S=2$. The kinetic energy $E_{K}$ of an $S_{2}$ electron is

$$
E_{K}=\left(N_{0}+S\right) \hbar \omega-E_{B}=25 \mathrm{eV}-13.6 \mathrm{eV}=11.4 \mathrm{eV}
$$

The energy that an electron spectrometer measures may be lower than $E_{k}$. This is because the electron quivers in response to the oscillating electric field. The energy of this quivering motion may be converted to energy of linear motion if the electron emerges from the field in a time that is short compared to the pulse length, in which case the spectrometer will measure $E_{K}$. If the pulse amplitude decreases while the electron is within the field, however, the quiver energy will be transferred to the field, and the electron's final kinetic energy will be less than $E_{K}$. This quiver energy, usually termed the "ponderomotive potential", is discussed further in Chapter 2. 


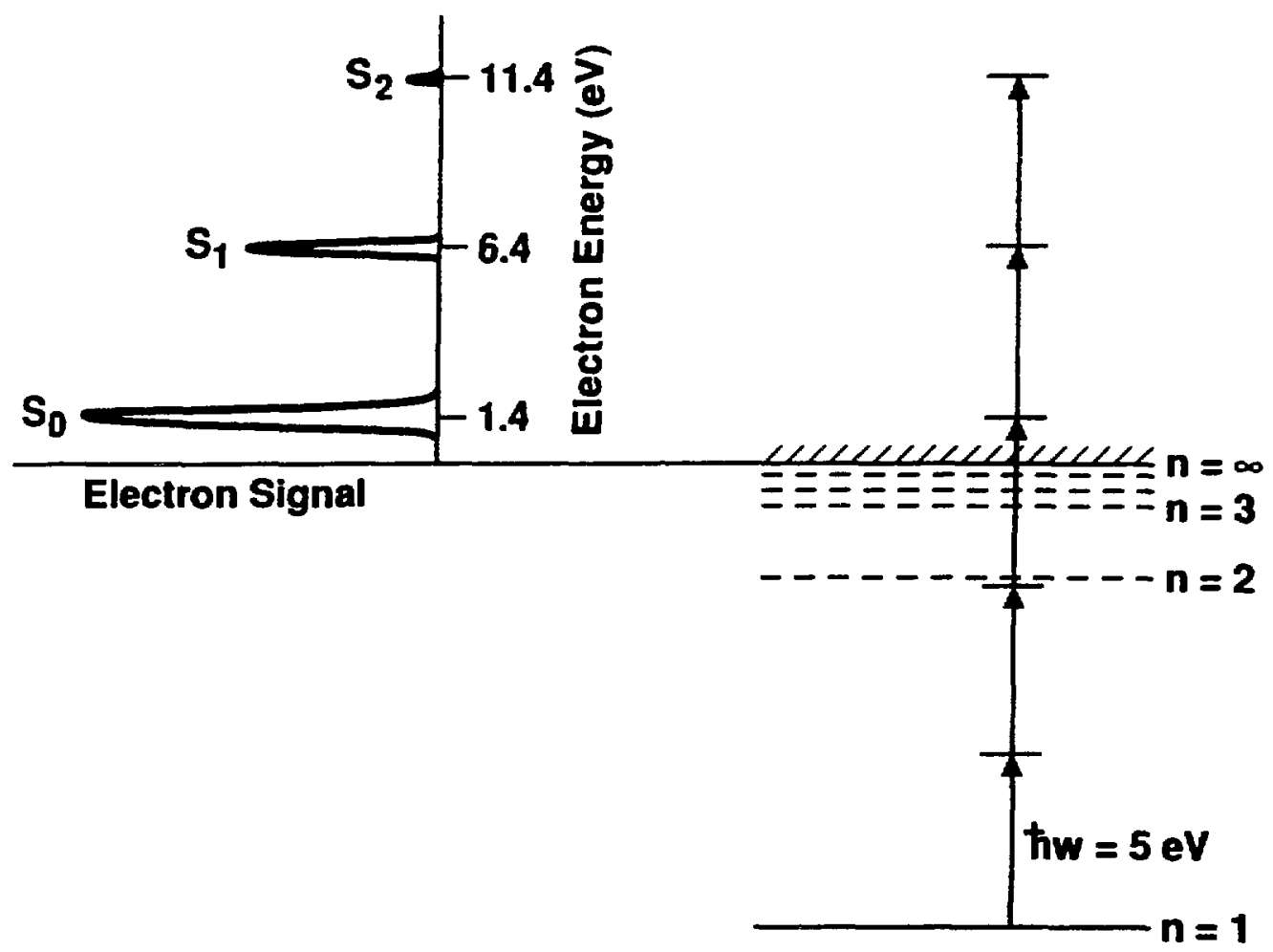

Figure 1. Simplified energy level diagram of a hydrogen atom showing electron peaks from absorption of three, four, and five $5 \mathrm{eV} \mathrm{KrF}$ photons.

\subsection{Experiments in ATI}

Interest in strong field experiments preceded the means to perform them by many years. Almost as soon as he had defined the photon, Einstein suggested that multiphoton processes should occur (Einstein 1909). A quantum theory of two-photon absorption was published in 1931 (Göppert-Mayer). This theory, however, showed that measurable rates of multiphoton absorption require light fields far more intense than any available at that time. When the laser made such fields available, they were quickly applied to MPI. With each increase in applied intensity, new measurements were compared with the predictions of LOPT. 
Eventually, the limits of LOPT were found. Beyond them lay the province of ATI.

ATI was probably observed in 1976 , but the data were misinterpreted until 1979 (Agostini and Petite 1988). Multiphoton ionization at irradiances of $10^{15} \mathrm{~W} / \mathrm{cm}^{2}$ between 1976 and 1979 produced electrons with many times the photon energy (Martin and Mandel 1976, Hollis 1978, Boreham and Hora 1979) - ionization of helium with $1.17 \mathrm{eV}$ photons produced $100 \mathrm{eV}$ electrons. The spectra could not be read clearly, however, and it was assumed that the electrons were accelerated from low to high energies by classical ponderomotive forces as they left the focal region. These ponderomotive forces had been described by Kibble and coworkers (Brown and Kibble 1964, Kibble 1966a,b). The now-familiar pattern of distinct peaks separated by the photon energy was first resolved in six-photon ionization of xenon at $10^{11} \mathrm{~W} / \mathrm{cm}^{2}$ with $2.34 \mathrm{eV}$ photons (Agostini et al. 1979). It was realized then that the ionization process was the source of the high energies, while the ponderomotive force simply transformed the quivering motion into linear translation.

The advent of high resolution electron spectrometers, some with solid angles of collection approaching $2 \pi$ sr (Kruit and Read 1983), allowed the high irradiance region to be reexamined (Kruit et al. 1983, Yergeau, Petite, and Agostini 1986), and there were further surprises. Not only did large numbers of peaks appear, but many of the low encrgy peaks vanished - as many as 2000 in experiments using $\mathrm{CO}_{2}$ lasers, judging from the absence of electron energies below about $100 \mathrm{eV}$ from xenon at $10^{13} \mathrm{~W} / \mathrm{cm}^{2}$ (Xiong, Yergeau, and Chin 1988). This phenomenon, known as peak suppression, was more pronounced when the light was circularly, rather than linearly, polarized (Bucksbaum et al. 1986). In contrast, spectra taken with $\mathrm{KrF}$ lasers (Luk et al. 1987) showed only a few peaks and no peak suppression at the same or higher irradiances. Rates of growth of the individual peaks with irradiance were generally found to be less than the $N_{0}+S$ 
power of irradiance expected from LOPT (Humpert et al. 1985). ATI electron peaks produced by picosecond-scale light pulses were shifted to lower energies compared to those produced by nanosecond pulses (Luk et al. 1987, Freeman et al. 1987). Experiments with pulses much shorter than a picosecond in xenon (Freeman et al. 1987) and in atomic hydrogen (Rottke et al. 1990) showed subpeaks which were duplicated within each primary peak and which corresponded to the energy levels of the target atoms.

\subsection{Comparisons to ATI Theory}

These and other strong-field experiments have made it possible to test many new approaches to nonperturbative calculations, and have demonstrated effects of the ponderomotive potential that could only be predicted a few years ago. However, none of them was intended to compare absolute numbers of ionizations to theoretical predictions of the ionization rates. Most used inert gases as targets, thus simplifying the experiment but complicating the theory. In most cases, atomic energy levels were near resonance with the light field, or passed through resonance because of the ac Stark shift as the intensity increased. This, too, required a sophisticated theory which had not yet been tested in a nonresonant case. Finally, only one group (LuVan et al. 1973) working with atomic hydrogen measured absolute numbers of ionizations. The others were interested primarily in the ac Stark shift (Kelleher, Ligare, and Brewer 1985), ionization by two applied radiation fields (Muller, van Linden van den Heuvell, and van der Wiel 1986), or angular distributions of the resulting electrons (Feldmann et al. 1987, Wolff et al. 1988, Rottke et al. 1990). When LuVan et al. measured an absolute ionization rate at $\lambda=0.53 \mu \mathrm{m}$, they could not compare it directly to theoretical predictions, because their laser field was multimode. Fluctuations in irradiance caused by mode beating increased the ionization rate by a factor which could not be measured, but which they estimated to be $N_{0} !=720$, which is the limiting value for an infinite number of uncorrelated modes. A small error in this factor 
would have had a large impact on the comparison to theory. In addition, working before the discovery of ATI, they collected ions instead of electrons and did not separate contributions from different ATI channels.

The present experiment was intended to provide well-calibrated counts of ATI electrons without the complications of complex targets and transient resonances. It employed a modelocked laser which produced a subpicosecond pulse with a smooth temporal shape and a well-defined peak irradiance. A theory which performs well in this case can then be tried under more difficult conditions. The following sections discuss the problems that have been avoided or mitigated in the present work, then outline the process by which measured electron spectra are compared to theoretical calculations.

\subsubsection{Selection of target gas}

It is desirable to avoid two disadvantages of inert gas targets: their complicated wave functions and the possibility of exciting two or more electrons simultaneously. Collective excitations are an interesting subject, to be sure. If entire shells of electrons can be excited, it might be possible to remove electrons from deep levels (Białynicka-Birula and Białynicki-Birula 1986). Still, theories of such complicated interactions must first be proved in simple systems, then applied to inert gases. Excitations of single electrons can be studied in numerical integrations of the Schrödinger equation by using a Hartree-Fock approach to account for the response of the other electrons (Kulander 1988), but these calculations cannot easily be applied to cases such as ATI of krypton that leave the ion in an excited state (Kulander 1987b, Lambropoulos 1988).

On the other hand, inert gas targets have the advantage of providing a low density target with no molecular component. Low density is important to prevent modification of the electron spectrum by collisions and by space charge, as well as to avoid overloading the electron detector. It is best to have just one kind of target in the interaction region because extra peaks make the electron spectra 
harder to analyze, especially as each peak broadens with intensity. This problem is more severe when the unwanted targets are molecules. Molecules produce wider and more numerous peaks than atoms because of the larger number of final states, including vibrationally excited and dissociated states. In addition, extra targets contribute to the space charge without increasing the signals of interest.

Atomic hydrogen can be substituted for the inert gas target if attention is given to regaining some of the lost advantages. Low density can be achieved through differential pumping with high-speed pumps. This also suppresses extra peaks from background gases. A molecular hydrogen component is unavoidable, but it can be minimized by keeping the discharge tube clean and using the fastest possible pump to remove recombined atoms from the interaction region. Fortunately, most of the $\mathrm{H}_{2}$ electrons can be distinguished from $\mathrm{H}$ electrons by their energies, and they provide the scaling factor needed to subtract the others from the total electron count.

\subsubsection{Resonances}

The rate of multiphoton absorption may be much higher for laser frequencies near an atomic resonance than for those farther away. The $2 \mathrm{~s}$ level of atomic hydrogen, $10.2 \mathrm{eV}$ above the ground state, is far enough from resonance with $5 \mathrm{eV}$ photons that it cannot be populated to any significant extent, but the multiphoton ionization rate with linear polarization is still enhanced by a factor of five. This can be seen in comparison to calculations with circular polarization, which cannot excite the 2s state. LOPT predictions for MPI of atomic hydrogen by linearly and circularly polarized fields appear as Fig. 2 (Maquet 1977).

As intensities increase at the start of a laser pulse, the energies of atomic states change because of the ac Stark effect. This may bring a particular state nearer to resonance with the field, or take it farther away. Generally, higher energy states shift away from the ground state. A classicion explanation for this 


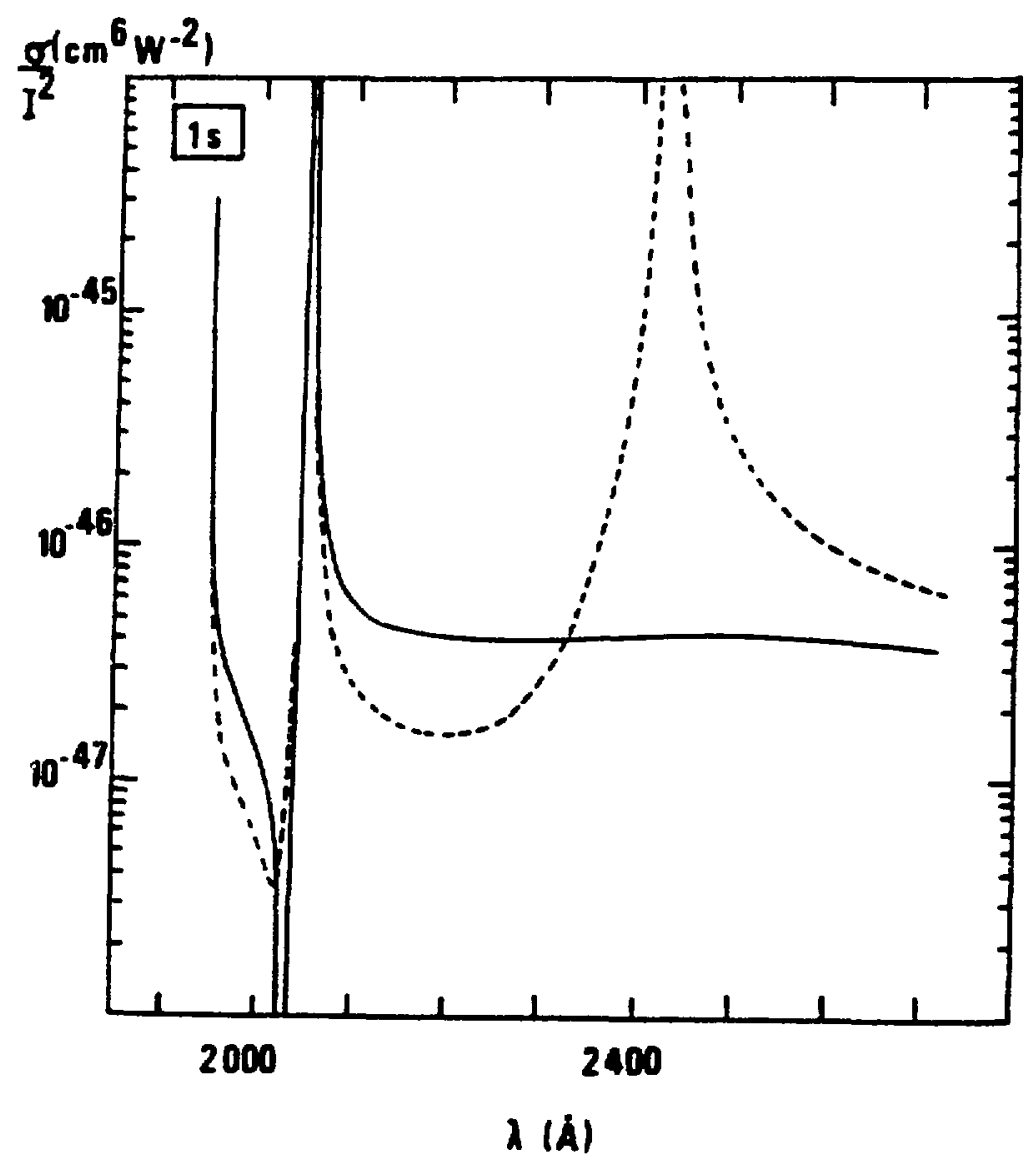

Figure 2. Three-photon ionization rates from the hydrogenic state 1s. Solid line: circularly polarized light; dashed line: linearly polarized light. (Maquet 1977)

is that electrons in larger orbits move farther along the electric field of the light, which is nearly constant over atomic dimensions. The light field therefore perturbs their energy more. Figure 3 shows the calculated behavior of atomic hydrogen in fields up to $2 \times 10^{14} \mathrm{~W} / \mathrm{cm}^{2}$. The atomic levels broaden as well as shift because their lifetimes become short.

Since the 2 s energy is initially greater than $2 \hbar \omega$, the ac Stark shift makes the ionization process less resonant as the field increases. At high enough field strength, the broadened $2 \mathrm{~s}$ state overlaps the $10 \mathrm{eV}$ line, but by that time in the pulse there may be no neutral atoms left. This situation is somewhat different 


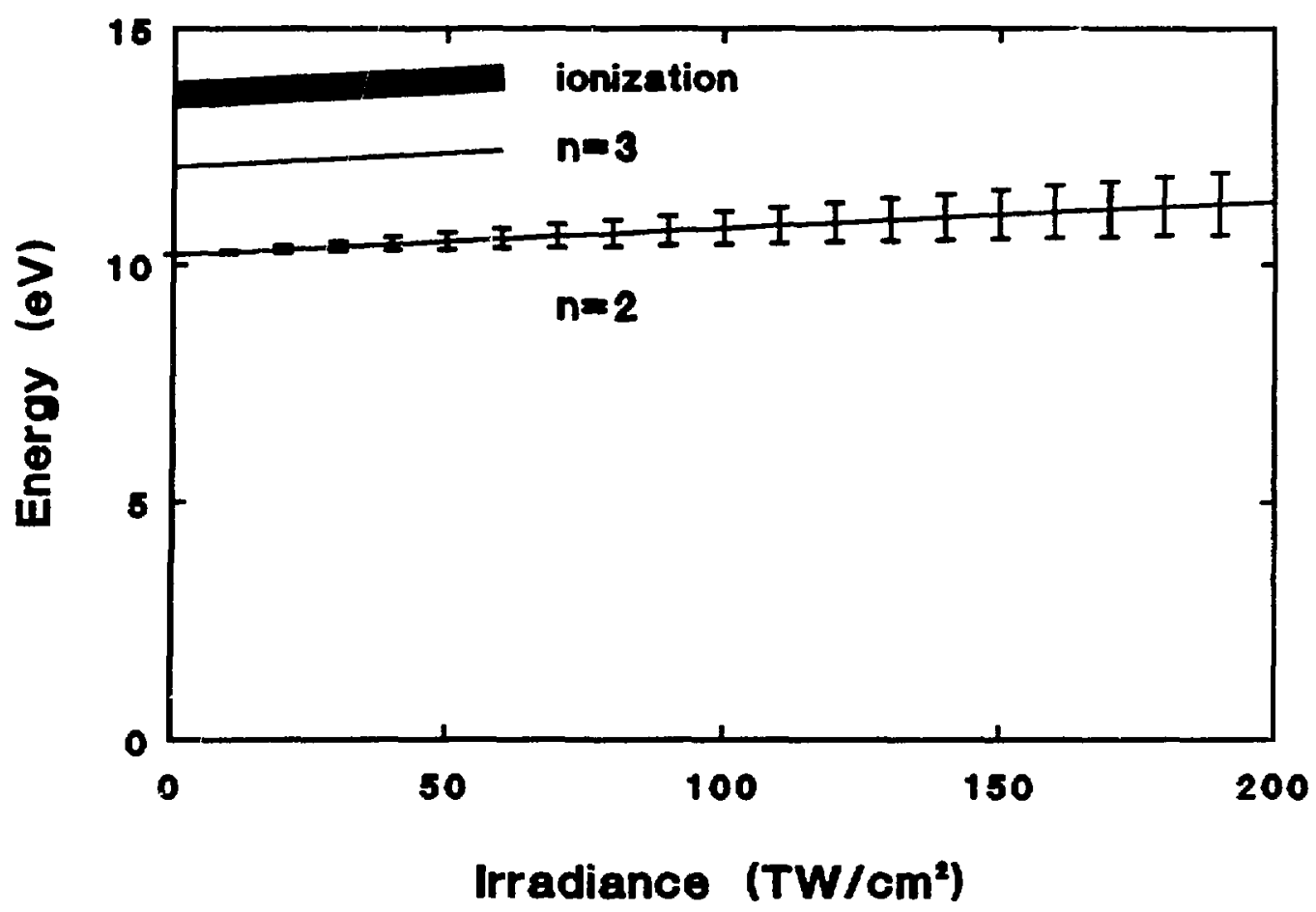

Figure 3. Shifting and broadening of atomic hydrogen energy levels by a strong $\mathrm{KrF}$ laser field. The shifts of the excited states are approximately equal to the ponderomotive potential. The shift of the ground state is negative and much smaller. The width of the $n=2$ level is calculated from an ionization cross section of $8.5 \times 10^{-18} \mathrm{~cm}^{2}$ (Downey and Hozack 1989).

from the "nonresonant" ATI of hydrogen experiment of Wolff et al. (1988)(quotes theirs). Their highest photon energy was $3.5 \mathrm{eV}$. The $2 \mathrm{~s}$ level therefore started $0.3 \mathrm{eV}$ below $3 \hbar \omega$. It would shift into resonance at an intensity of approximately $3 \times 10^{14} \mathrm{~W} / \mathrm{cm}^{2}$, and strongly affect the ionization rate at lower intensities.

\subsubsection{Electron counts}

Theoretical calculations produce a curve of ionization rate as a function of instantaneous irradiance, averaged over an optical cycle. Most models do not consider the past history of the atom, though some numerical integrations of the 
Schrödinger equation have included ramped as well as instantaneously applied fields and have found no significant difference. Experiments, on the other hand, result in total numbers of electrons produced by laser pulses of a certain spatial and temporal shape with various peak irradiances. Electrons are produced in the low-irradiance wings of the focus as well as at the center. They are produced early in the pulse as well as later. If all of the atoms at the center of the focal volume are ionized before the end of the pulse, no electrons are produced there at later times.

In order to compare measurements against a theory, one must fold the predicted ionization rates together with the experimental parameters to predict a signal. One must consider the distribution of irradiances in time and space, the density of atoms throughout the focal volume at each time, and the characteristics of the experimental apparatus. After this, it remains to identify and count the electrons from the time-of-flight spectrum that correspond to the modeled process.

We folded the models with the experimental parameters by dividing the focal volume into shells along contours of equal peak irradiance, so that all atoms within a shell experienced the same temporal history. Some shells were truneated at the edge of the atomic beam, but no further truncation was necessary to account for the collection limits of the electron spectrometer. Within each shell, we integrated the theoretical ionization rates over the temporal shape of the laser pulse to find the number of electrons released. These results were summed over the entire focal volume to obtain the predicted electron count. Chapters 3 and 4 and the appendices describe how the relevant experimental parameters were measured, and how they were used in comparing the models to the data. 


\subsection{Questions to be answered by this work}

The primary result of this work is a comparison of total rates of nonresonant multiphoton ionization to theoretical predictions for atomic hydrogen, using linearly polarized $248 \mathrm{~nm}$ light at irradiances as high as $2 \times 10^{14} \mathrm{~W} / \mathrm{cm}^{2}$. The total rate of ionization was found from the number of electrons produced, regardless of which ATI channel they occupied, and the density of target atoms. It is an absolute rate, not a relative rate, meaning that the actual numbers of electrons and atoms are used.

The other result of this work is a comparison of the partial rates $R_{0}$ and $R_{1}$ of ionization into the $S_{0}$ and $S_{1}$ ATI channels. Each partial rate $R_{S}$ is described by an index of nonlinearity

$$
K_{S}=\frac{\partial \ln R_{S}}{\partial \ln I}
$$

where $S$ and $I$ have been defined previously. At low irradiances, $K_{S} \approx N_{0}+S$. This means that the higher order ATI peaks tend to overtake the lower order peaks in size as the irradiance increases. The branching ratios among the peaks thus provide a test of the theories. Also, values of $K_{S}$ tend to decrease with irradiance, so they are of interest themselves. In measurements of $K_{S}$ using xenon atoms and $1.06 \mu \mathrm{m}$ light, the index of nonlinearity starts around 11 for $S=0$ and increases with $S$. In these experiments, $K_{0}$ starts at 3 , allowing more tolerance for uncertainties.

The above results are supported by measurement of the irradiance at which "depletion" occurs. This is the point, mentioned before, at which all atoms at the center of the focal volume are ionized. Further increasing the irradiance produces more electrons, but only because the low-irradiance wings of the focus expand to include more atoms. The order of nonlinearity of each peak is then governed by the geometry of the experiment (Cervenan and Isenor 1975, Sogard 1988). For cylindrically symmetric laser beams, uniform atomic densities, and wide field of 
view electron detectors, all of the $K_{S}$ become 1.5 regardless of the order of the ionization process. In the present experiment, the order of nonlinearity becomes less than one when the focal volume expands beyond the limits of the atomic beam. When the depletion irradiance is known, approximate values of atomic density and ionization rate can be used to check the measured density and rate. Depletion also marks the highest irradiance that can be studied without reducing the rise time of the laser pulse. 


\section{Theory}

For many years, lowest order perturbation theory (LOPT) was adequate for multiphoton ionization calculations. In principle, inclusion of additional terms can provide results to any desired accuracy if the series converges. When many high order terms must be evaluated, however, the cost of computation becomes high. Other techniques may then become attractive. This chapter begins with pre-ATI strong field theory, then touches on refinements to perturbative calculations and nonperturbative techniques that have been applied to ATI.

\subsection{Strong Field Physics Before ATI}

\subsubsection{Perturbation Theory}

Multiphoton absorption, like so much of modern physics, was foreseen by Einstein (1909). The first calculation of absorption rates, however, was that of Maria Göppert-Mayer (1931). She used a term from the second order perturbation expansion, in powers of electromagnetic field strength, of the matrix element $\langle f|A(\infty)| i\rangle$. Here, in the notation of Faisal (1987), $A(t)$ is the evolution operator which takes an initial state $|i\rangle$ at time zero into the state $|t\rangle$ at a later time $t$ :

$$
|t\rangle=A(t)|i\rangle
$$

The expansion of $A(t)$ is

$$
A(t)=1+\sum_{N=i}^{\infty} A^{(N)}(t),
$$

where each term $A^{(N)}$ is proportional to the $2 N$ power of the field amplitude $E$. In order to study the state of the electron after the interaction is finished, one takes the limit $t \rightarrow \infty$. The projection onto the final state $|f\rangle$ is then

$$
\lim _{t \rightarrow \infty}\langle f \mid t\rangle=\langle f|A(\infty)| i\rangle
$$


The part of the second order term $A^{(2)}$ representing absorption of two photons is

$$
A_{a a}^{(2)}=\frac{-2 \pi i}{\hbar^{2}} \delta\left(\omega_{i}-\omega_{f}+2 \omega\right) \frac{\sum_{j}(E / 2)\left\langle f\left|D_{\lambda}\right| j\right\rangle(E / 2)^{*}\left\langle j\left|D_{\lambda}\right| i\right\rangle}{\left(\omega_{i}+\omega-\omega_{j}\right)}
$$

In this expression, the electric field is

$$
\mathrm{E}(t)=\frac{\mathbf{E}}{2} e^{-i \omega t}+\frac{\mathbf{E}^{*}}{2} e^{i \omega t}
$$

in the dipole approximation, and the interaction energy is

$$
D_{\lambda}=\hat{\epsilon}_{\lambda} \cdot \mathbf{D}
$$

where

$$
\begin{aligned}
& \hat{\epsilon}_{\lambda}=\text { polarization vector in mode } \lambda, \text { and } \\
& \mathbf{D}=\text { dipole operator of atom, }
\end{aligned}
$$

in the length, or E - r, gaugc, which was introduced in the same 1931 paper. The sum is over the complete set of atomic states $|j\rangle$ with energies $\hbar \omega_{j}$. The $\delta$-function imposes strict energy conservation in the limit of infinite time. It is integrated over a line shape or a density of continuum states in the course of computing a transition rate. Since $A^{(2)}$ is the first term in the expansion which contributes to the matrix element, it is the LOPT prediction for two-photon absorption. Similarly, the LOPT for a three-photon process would include only $A^{(3)}$.

One important feature of the two-photon LOPT solution $A_{a a}^{(2)}$ is the factor of $E^{2}$, which is proportional to the intensity $I . I^{2}$ appears when the matrix element is squared to obtain a probability. The LOPT rate for an $N$-photon process is always proportional to $I^{N}$.

Another important feature of $A_{a a}^{(2)}$ is its denominator, which may vanish for some intermediate states. This causes the absorption rate to be much higher for frequencies which resonate with allowed atomic energy levels. It was therefore possible to study resonant multiphoton ionization soon after lasers became 
available, whereas intensities high enough to produce nonresonant multiphoton ionization came several years later. Calculations of resonant MPI rates, however, require precise knowledge of the energies of the resonant states, and often encounter computational difficulties. These computational problems become more difficult when the sum over discrete states is extended to include an integral over continuum states above the ionization threshold, all of which are allowed levels. One way to proceed is to extract the integral representation of the Green's function, or propagator,

$$
G\left(\omega_{i} \pm \omega\right) \equiv \mathcal{F}_{j} \frac{|j\rangle\langle j|}{\left(\omega_{i} \pm \omega-\omega_{j}\right)}
$$

and replace it with one of several other representations that do not include an explicit integration.

Besides two photon absorption, the second order perturbation term $A^{(2)}(t)$ produces an amplitude for two photon emission and an amplitude for simulitineous absorption and emission of single photons of equal energy. The latter is

$$
\begin{aligned}
A_{a e}^{(2)}=\frac{-2 \pi i}{\hbar^{2}} \delta\left(\omega_{i}-\omega_{f}\right)\left[\left|\frac{E}{2}\right|^{2}\left\langle f\left|D_{\lambda} G\left(\omega_{i}+\omega\right) D_{\lambda}\right| i\right\rangle\right. & \\
& \left.+\left|\frac{E}{2}\right|^{2}\left\langle f\left|D_{\lambda} G\left(\omega_{i}-\omega\right) D_{\lambda}\right| i\right\rangle\right] .
\end{aligned}
$$

This term describes the ac Stark shift if the initial and final states are identical, or the mixing of degenerate states if they are not identical.

Second order perturbation theory is not a complete description of two photon absorption, of course. The $A^{(4)}$ term describes four processes which result in the net absorption of two photons, and so on. Until the invention of the laser, however, these contributions could not be made large enough to matter, and LOPT was quite satisfactory. 


\subsubsection{Ponderomotive Forces}

Ponderomotive forces are very important in strong field interactions. These appear to arise from a "ponderomotive potential" $U$, proportional to the irradiance, which pushes charged particles away from areas of high field strength. In atomic units,

$$
U=(E / 2 \omega)^{2}
$$

Atomic units are defined in Appendix A. In other units,

$$
U=9.30 \times 10^{-14} I \lambda^{2},
$$

where $U$ is in $\mathrm{eV}, I$ in $\mathrm{W} / \mathrm{cm}^{2}$, and $\lambda$ in $\mu \mathrm{m}$. In the present experiment, $\lambda=0.248 \mu \mathrm{m}$, so

$$
U=5.72 \times 10^{-15} I \text { for } \mathrm{KrF} \text {. }
$$

The ponderomotive force arises from the quivering motion of a charged particle in an ac field. During each half cycle, the particle is accelerated and decelerated by the field. If the field is uniform in space, it is possible for the particle quiver with zero average velocity, returning to its starting point at the end of each cycle. If, however, the particle's quivering motion takes it from a high field region into a low field region, the deceleration will not balance the acceleration, and the particle will not return to its starting point. On each subsequent half cycle, the particle will lose quiver velocity and gain linear velocity. There is thus a symmetry between electrons and photons in scattering processes (Kibble 1966a,b). Photons may be refracted by variations in electron density, and electrons may be refracted by gradients in the ac field.

Of course, the ponderomotive force is not a force under the usual definition, but the difference between forces experienced by a particle at different times in different places. Still, when the particle's position is averaged over an optical cycle, so that the quiver energy is not apparent, it is convenient to calculate 
ponderomotive forces. If the envelope of the field is constant, the ponderomotive force can be represented as the gradient of a ponderomotive potential equal to the kinetic energy of the quivering motion. If the envelope is not constant, the particle can exchange energy with the field. The potential picture is still practical, though, so long as the average field strength acting on the particle $\bar{E}(\mathbf{r}, t)$ changes more because of the particle's velocity $\mathbf{v}$ than because of the time-dependent envelope, that is, so long as

$$
\mathbf{v} \cdot \nabla \bar{E}(\mathbf{r}, t) \gg \partial \bar{E}(\mathbf{r}, t) / \partial t
$$

The ponderomotive potential is relevant to ATI in two ways. First, the initial distribution of electron trajectories near the point of ionization can be altered greatly by gradients in the light field. This obscures the angular patterns that result from resonances with atomic states of definite angular momentum. Second, every electron that emerges from an ionizing atom must possess energy at least equal to the ponderomotive potential. If this potential is great enough, it is impossible to produce $S_{0}$ electrons, and that peak in the ATI spectrum is suppressed. Greater potentials can then suppress additional peaks.

The ponderomotive potential model is a powerful conceptual tool, but one must always remember that it actually describes kinetic energy, not potential energy. If the electron leaves the field quickly compared to changes in the average field intensity (the long-pulse limit), the quivering motion is converted to linear motion, and the potential model is seldom misleading. A popular picture for this is a surfboard rider gaining speed by sliding down the side of a wave (Bucksbaum et al. 1987). In the opposite case (the short-pulse limit), the average intensity of the field vanishes before the electron escapes. This is similar to an ocean wave lifting a floating cork, thereby increasing its energy, then reabsorbing the energy as it moves away. In this case, the potential model must be used with care. The boundary between the two regimes falls in the picosecond range for typical focal 
spot sizes. In the present experiment, the spot radius is tens of micrometers, and the pulse length is 700 fsec FWHM. The speed of a $10 \mathrm{eV}$ electron is about $2 \mu \mathrm{m} / \mathrm{psec}$, so very few electrons can leave the focal region before the end of the pulse, making this is a short-pulse experiment.

\subsection{ATI Theory}

\subsubsection{Introduction}

Any perturbative calculation has a limited range of applicability. Often the limit has been reached when the expansion variable exceeds an atomic unit, so that higher order terms are not necessarily smaller than the LOPT terms, and it becomes difficult to determine whether enough of the infinite series has been summed. Appendix A contains several other conditions that must be satisfied if LOPT is to be valid. Perturbative calculations encounter other problems even at lower intensities, however. Somewhere around $10^{12} \mathrm{~W} / \mathrm{cm}^{2}$ it becomes impossible to limit the calculation to transitions among bound states and between bound states and the continuun. Continuum-continuum ( $\mathrm{C}-\mathrm{C})$ transitions must also be considered. The difficulty is that any energy in the continuum is an allowed energy, so there is nothing to prevent the detuning term in the denominator from becoming zero. Some method must be found to avoid explicit integration over the continuum states. This is actually a calculational problem, not a fundamental one, since the true ionization rates are not divergent (Agostini and Petite 1988). Indeed, one of the greatest puzzles in perturbation theory is why LOPT continues to work for total ionization rates long after the individual ATI peaks have become nonperturbative. What may be a more serious limitation on the current methods of extending perturbation theory is that they all use monochromatic (eternal) laser fields (Véniard and Piraux 1990).

An alternative to improving the techniques of perturbative calculation is to treat the strong field nonperturbatively. The most direct way is to solve the 
Schrödinger equation numerically, a feat which has become practical in the age of supercomputers. Another is to build wave functions from exact or approximate solutions for periodic fields, using the initial atomic state as a boundary condition.

Amazingly, the qualitative features of ATI emerge from almost any model that includes coupling among the continuum states and the ponderomotive potential. Even purely classical models (Kyrala 1987, Chu and Yin 1987) enjoy some success and help to visualize the prucess. Quantitative agreement has been more elusive. Clearly ATI is not such a simple process that its details may be discarded wholesale. The experimental measurements that are most sensitive to differences among the models are the total rates of ionization, the intensity dependencies of different ATI peaks, and the angular distributions of the electrons in each peak.

\subsubsection{Higher order perturbation theory}

Perturbation theory can describe interactions which produce ATI peaks as high as $S=s$ if the calculation extends to order $N_{0}+s$. Several ways of evaluating high-order terms have been reported (Aymar and Crance 1981; Gao and Starace 1988 and 1989; Gao, Pan, Liu and Starace 1990; Gontier and Trahin 1968 and 1989; Gontier, Rahman and Trahin 1986 and 1988; Maquet 1977; Karule 1975, 1988, 1990; Potvliege and Shakeshaft 1989a; Shakeshaft 1986). Most of these calculations use some representation of the Coulomb Green's function, as described in section 2.1.1, to avoid numerical problems in integrating over the continuum, but they still face problems of determining whether the perturbation series has converged, or will converge, and they become increasingly expensive to carry out as the number of terms grows. (Recall that retaining $A^{(4)}$ in the two-photon absorption problem requires calculations for four new processes, not one.) 


\subsubsection{Numerical integration of the Schrödinger equation}

This is a straightforward technique that has the advantage of treating ATI as a coherent process (Lambropoulos, 1988). There are different approaches to implementing it, however. LaGattuta $(1990 \mathrm{a}, \mathrm{b})$ and Kulander $(1987 \mathrm{a}, \mathrm{b}$ and 1988) work in three dimensional coordinate space with distant boundaries to divide the continuum into discrete levels. (Cylindrical symmetry in the case of linearly polarized radiation and ground-state hydrogen allows the calculation to be done on a two-dimensional lattice.) They obtain good angular resolution, but limited energy resolution (Kulander 1988). Collins and Merts (1990) prefer three dimensional momentum space with a Volkov basis set, since the electrons have definite momenta and indefinite positions at late times. There have also been several solutions of the one dimensional Schrödinger equation, the first by Javanainen, Eberly, and Su (1988, and Javanainen and Eberly 1988). These integrations are easier to carry out for high irradiances than for low, since fewer time steps are required (Kulander 1987a). For this reason, tabulated ionization rates for atomic hydrogen at $248 \mathrm{~nm}$ do not extend below $10^{13} \mathrm{~W} / \mathrm{cm}^{2}$. Comparisons between these rates and measured electron counts, however, are very sensitive to the missing values, since large volumes of hydrogen in the wings of the focal volume are ionized at low irradiances.

\subsubsection{Keldysh-Faisal-Reiss (KFR) models}

In KFR (Keldysh 1965, Faisal 1973, Reiss 1980, Reiss 1987, Reiss 1990) models, the electron leaps directly from a bound state unaffected by the external field to a Volkov plane wave state (Volkov 1935) affected only by the external field. These models reproduce all of the features now understood to result from C-C transitions, but they predict ionization rates much smaller than those that have been measured.

KFR models use the Volkov wave function to represent the final state of an ionized electron in an S-matrix formalism. The plane wave Volkov state 
$\psi_{e}(\mathbf{x}, t)$ satisfies the Schrödinger equation for an electron in a monochromatic field, described by the vector potential $\mathbf{A}(t)$, in the absence of other potentials. In the $\mathbf{E} \cdot \mathbf{r}$ gauge,

$$
\begin{aligned}
\psi_{e}(\mathbf{x}, t)=\exp [-i( & \frac{\mathbf{p}^{2}}{2 m} t-(\mathbf{p}-e \mathbf{A}(t)) \cdot \mathbf{x} \\
& \left.-\frac{1}{2 m} \int^{t} d \tau\left(2 e \mathbf{p} \cdot \mathbf{A}(\tau)-e^{2} \mathbf{A}^{2}(\tau)\right)\right]
\end{aligned}
$$

where $\mathbf{p}$ is the eigenvalue of the canonical momentum operator in the $\mathbf{p} \cdot \mathbf{A}$ gauge. This solution is valid for fields of arbitrary strength, so long as the electron does not reach relativistic speeds (see Appendix A), and so long as it is used in a way consistent with the dipole approximation $\mathbf{A}(\mathbf{r}, t) \approx \mathbf{A}(t)$ (Reiss 1990). This electron wave function is combined with the initial atomic wave function $\psi_{A}$ and the final ionic wave function $\psi_{A^{+}}$, where appropriate, to form the matrix element

$$
\mathrm{M}=\left\langle\psi_{A}+\psi_{e}\left|V_{A}\right| \psi_{A}\right\rangle
$$

where $V_{A}$ is the potential energy due to the applied field. $M$ is then used to derive the S-matrix and the transition rate. The presence of the bound state wave function $\psi_{A}$ in $\mathrm{M}$ is what justifies using the dipole approximation for $\psi_{\mathrm{e}}$ (Reiss 1990). The fact that $M$ can be evaluated analytically is the major advantage of KFR models. When $\psi_{A}$ is spatially symmetric, the only property of $\psi_{A}$ appearing in the transition rate is the binding energy $E_{B}$. The relatively simple predictions of KFR theory are frequently used in calculating heating rates due to ATI in plasmas (Corkum, Burnett, and Brunel 1989).

One variation on the KFR model (Becker, Schlicher and Scully 1986) simplifies the situation even more. The entire spatial integration in the matrix element $\mathrm{M}$ is replaced by a factor proportional to $I^{N / 2}$. Nothing remains of the initial state of the atom or of the interaction potential between the atom and the field. Whatever results from the integration over time is therefore due to $\mathrm{C}-\mathrm{C}$ coupling. 
Despite its simplicity and its disregard of the angular momentum carried by the electron, this model predicts the distribution of electrons among the ATI peaks fairly well (Petite, Agostini, and Muller 1988).

As mentioned before, KFR models underpredict ionization rates, but the discrepancy might be expected to be less in the case of $\mathrm{KrF}$ light and hydrogen targets than in some other cases. The underprediction is caused, at least in part, by the difficulty in satisfying the assumptions of the model when neutral atoms are ionized. These are that the supply of atoms is not depleted, no resonances occur with other bound states of the atom, and the binding potential is shortrange (Reiss 1980). The first condition is satisfied in the present experiment only at the lowest irradiances, and the second is not fully satisfied even then. The third condition is never satisfied in ionization, since the Coulomb potential has infinite range. In the present experiment, however, resonances are not expected to dominate the process, and the effect of the Coulomb tail on the final state is reduced by the fact that even the slowest electrons move at speeds of $3 \times$ $10^{7} \mathrm{~cm} / \mathrm{sec}$. KFR models might perform well in such a case (Sundaram and Armstrong 1990), especially if a semiclassical factor

$$
F_{C}=A E_{B} \gamma / \hbar \omega\left(1+\gamma^{2}\right)^{1 / 2}
$$

derived by Keldysh is included to account for the Coulomb field. This factor, with an appropriate value of $A$, brings the Keldysh model in the tunneling limit $(\gamma \ll 1)$ into agreement with the accepted rate of tunneling ionization in a dc field (Keldysh 1965). In the case of atomic hydrogen and $\mathrm{KrF}$ radiation, $A=13$ (Baldwin and Boreham 1981), and $F_{C} \approx 35$ for irradiances below $10^{15} \mathrm{~W} / \mathrm{cm}^{2}$. Recent work in the KFR area has concentrated on replacing the Volkov final state with one that more closely resembles free electrons in a Coulomb field (Leone et al. 1987, Shakeshaft and Potvliege 1987, Pan 1989). 


\subsubsection{Floquet calculations}

Another method of obtaining wave functions that reflect the periodicity of the laser field is based on Floquet's 19th century studies of differential equations with periodic coefficients. Applied to quantum mechanics (Shirley 1965), the wave function $\chi(x, t)$ describing an atom in a laser field is factored as

$$
\chi(x, t)=e^{-i \beta t} \psi(x, t)
$$

where $\psi(x, t)$ is periodic in $t$. In this factorization, $\beta$ is a complex "energy" that describes both the total energy of the system and the decay rate of the atomic state. (In order to have real energy eigenvalues, one must construct a wave packet (Potvliege and Shakeshaft 1989b)). The $\psi$ functions satisfy a time-independent equation like the Schrödinger equation, but with a non-Hermitian hamiltonian. The function $\psi(x, t)$ is further divided into Fourier components, each of which has the complex energy

$$
E_{c}=\beta+N \hbar \omega
$$

where $\beta$ is determined by the atomic state. The value of $\beta$ varies as the atom is "dressed" by a photon field of increasing strength, with different atomic levels varying at different rates. Transitions may occur between states of different $\beta$ and $\mathrm{N}$ if the total energies cross. These are absorptions or emissions of real photons. Some crossings are real, and some are avoided, leading to sensitivity to the rate of change of the field (adiabatic versus nonadiabatic transitions) (Crance 1988).

The advantage to the Floquet approach is that all transitions are included in the calculation, in principle, regardless of the number of photons exchanged. In practice, the largest change of $\mathrm{N}$ in the calculation is increased until the solution converges. This is equivalent to a perturbation calculation that extends to all orders in field strength. The precision of the atomic wave function must 
also be limited in some way. Chu and coworkers use eigenvectors of total angular momentum $\mathrm{L}^{2}$ as a discrete atomic basis, including higher values of $\mathrm{L}$ until the solution converges (Chu and Reinhardt 1977, Chu and Cooper 1985). Shakeshaft and coworkers use polynomial representations of the wave function on a continuous basis (Potvliege and Shakeshaft 1989b).

Floquet results for atomic hydrogen at $248 \mathrm{~nm}$ have been found to agree well with numerical integrations (Pindzola and Dörr 1990) between $10^{13} \mathrm{~W} / \mathrm{cm}^{2}$ and $10^{15} \mathrm{~W} / \mathrm{cm}^{2}$. These calculated rates increase less quickly than those of LOPT.

\subsubsection{Other methods}

Deng and Eberly (1985) calculated $\mathrm{C}-\mathrm{C}$ transitions among featureless continua. They found that these can change from incoherent to coherent processes at high irradiance, providing a way for electrons to pass by the lower energies and pool at the end of the coherent chain. These and other "essential state" calculations have not yet achieved high accuracy (Agostini and Petite 1988), perhaps because Coulomb systems do not have featureless continua (Véniard and Piraux 1990).

An entirely different way to study a quivering electron is to transform to an accelerated reference frame in which a free electron may be at rest, or move aiong a smooth trajectory. In this frame, the atomic potential varies at the optical frequency, and the Kramers gauge appears in the wave functions instead of the $\mathbf{E} \cdot \mathbf{r}$ or $\mathbf{p} \cdot \mathbf{A}$ gauges. Henneberger (1968) first applied this technique to multiphoton problems. It is more easily used at microwave or $\mathbf{x}$ ray wavelengths than in the UV (Walet 1990), but it has been used for numerical integration of the Schrödinger equation in one dimension at the $\mathrm{KrF}$ wavelength (Reed and Burnett 1990). 


\subsection{Predictions from the theories}

Figure 4 presents predictions from several models which have been exercised for atomic hydrogen and $248 \mathrm{~nm}$ radiation. These are values of ionization rate vs. instantaneous irradiance. The highest curve in Fig. 4 is one evaluation of LOPT (Khristenko and Vetchinkin 1976), which predicts an $I^{3}$ dependence on irradiance. The lowest curves represent the Keldysh and Reiss models with pure Volkov final states. Between these extremes are the Floquet results of Chu (1990) and the Keldysh model including Keldysh's quasiclassical correction for Coulomb field effects on the final state. The Coulomb-corrected Keldysh model is evaluated from equation 1 of Keldysh's paper (1965), the uncorrected model from equation 16 (which contains a misprint). Table 1 shows how the LOPT curve of Fig. 4 compares to calculations of Gao and Starace (1989), Gontier and Trahin (1968), Laplanche et al. (1976), and Maquet (1977). Since all LOPT models have the same dependence on irradiance, each can be represented by the generalized cross section in Table 1. Differences among these perturbation calculations are greater than a factor of two. It is clear from Fig. 4 that there are significant differences among the predictions of the different models that must be resolved before any of them can be relied upon in calculations of strong-field effects.

Table 1. Comparison of generalized cross sections calculated by LOPT for MPI of atomic hydrogen by linearly polarized $248 \mathrm{~nm}$ light.

\begin{tabular}{ll}
\hline \hline$\sigma^{(3)}\left(\mathrm{cm}^{6} \mathrm{~W}^{-2}\right)$ & Reference \\
\hline $4.2 \times 10^{-46}$ & Gontier and Trahin 1968 \\
$7.5 \times 10^{-46}$ & Laplanche et al. 1976 \\
$5.7 \times 10^{-46}$ & Khristenko and Vetchinkin 1976 \\
$5.0 \times 10^{-46}$ & Maquet 1977 \\
$3.1 \times 10^{-46}$ & Gao and Starace 1988 \\
\hline \hline
\end{tabular}




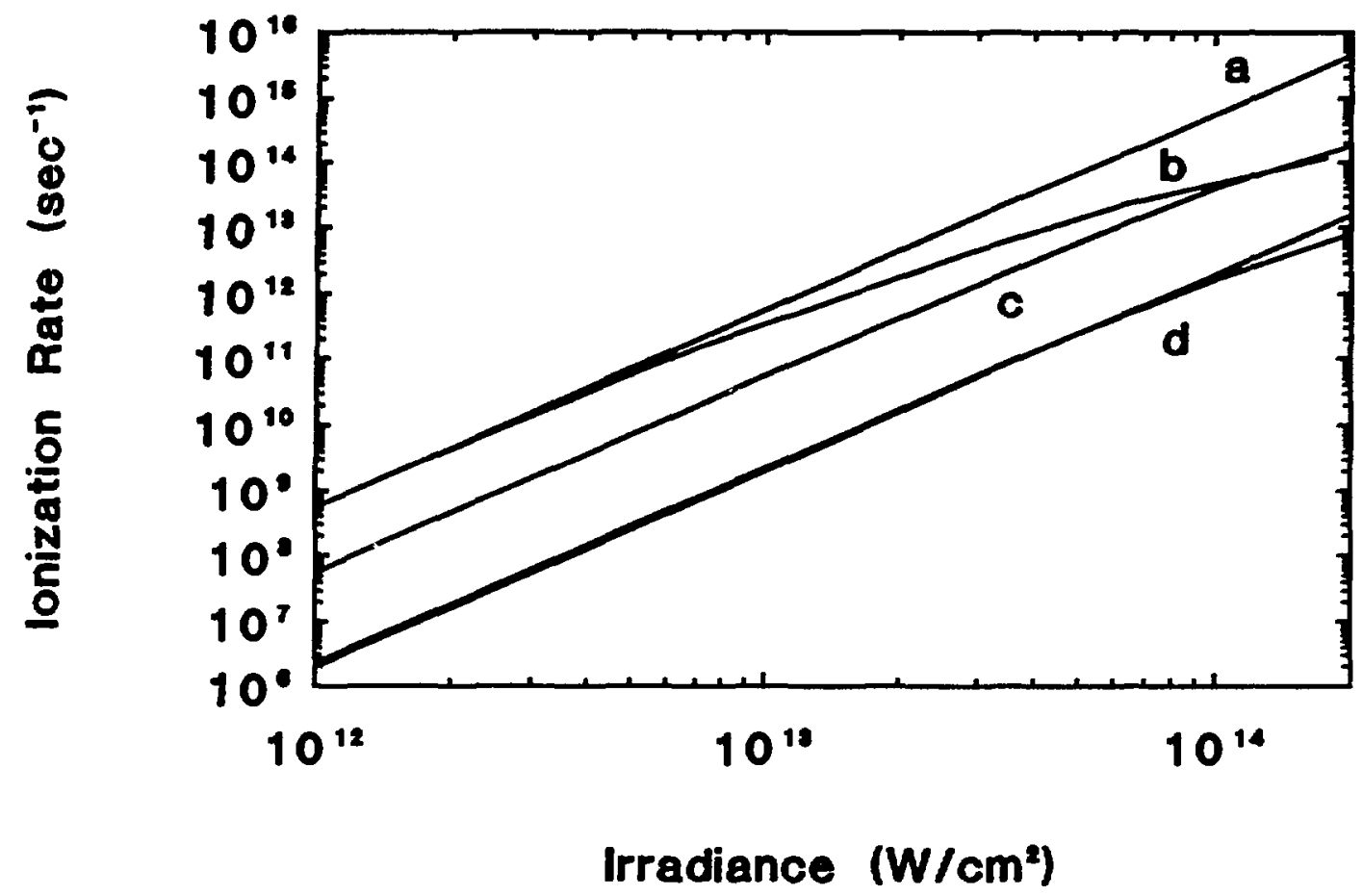

Figure 4. Predictions from five models of MPI rates for atomic hydrogen and linearly polarized $248 \mathrm{~nm}$ light. Curve (a) is LOPT evaluated by Khristenko and Vetchinkin (1976). Curve (b) is the Floquet theory of Chu (1990). Curve (c) is the Coulomb corrected Keldysh model (1965). Curves (d) are the Keldysh (1965) and Reiss (1980) models without Coulomb correction.

Before these ionization rates can be compared to experimental data, they must be converted to total numbers of ionizations as functions of the peak irradiance of the laser pulse, considering the spatial and temporal distribution of irradiance in the interaction region and the hydrogen atom density within the atomic beam. This conversion process is described in Chapter 4. 


\section{Description of the experiment}

The object of the experiment was to measure absolute rates of ATI by recordin $f$ the energy spectra of electrons from crossed beams of hydrogen $(H)$ atoms and ultraviolet light. This required a vacuum system, an optical system, a source of hydrogen atoms, an electron time-of-flight spectrometer, and electronics to process and store the spectrometer signal. We calibrated the apparatus to obtain the density of atoms and the actual number of electrons produced at the interaction region, and we determined how the crossed beams overlapped so that the measured ionization rates could be compared to the theoretical rates. This chapter considers each section of the apparatus, describing its function and its construction. It then covers procedures of calibration and operation. Finally, it shows that several factors that could potentially have affected the experiment were in fact not of concern.

\subsection{Overview}

An ultrahigh vacuum (UHV) maintained around the interaction region during the experiment minimized the electron signals from ionization of background gases, principally hydrogen molecules $\left(\mathrm{H}_{2}\right)$ and water. Pressures within the hydrogen beam at the interaction point were approximately $10^{-6}$ Torr. Differential pumping allowed the hydrogen source to operate at the higher pressure required for a radio frequency (rf) discharge. Gas pressure and composition in the interaction region were monitored by a quadrupole residual gas analyzer (RGA) under computer control. Hydrogen pressures at the RGA were converted to density in the interaction region.

The Los Alamos Bright Source I (LABS-I) wer jystem provided $40 \mathrm{GW}$ subpicosecond pulses at $248 \mathrm{~nm}$ wavelength. The pulse length of $600 \mathrm{fsec}$ full 
width at half maximum (FWHM) was near the transform limit. Polarization was linear. An off-axis paraboloid mirror working at $f / 20$ produced an eight to ten times diffraction limited focus. The rms irradiance at focus could be as high as $1.8 \times 10^{15} \mathrm{~W} / \mathrm{cm}^{2}$, which is 0.05 atomic unit. Lower irradiances were obtained with beam splitters and neutral density filters in order to change the pulse shape and irradiance distribution as little as possible. Even so, the presence of so many surfaces in the optical path may have contributed significantly to the size of the focal volume. Pulse energies could be measured individually, but pulse length and focal spot size could not. Fortunately, the electron spectra contain information about the irradiance level that assists in comparing data sets.

The spectrometer used a magnetic bottle arrangement to guide the electrons to a microchannel plate (MCP) detector assembly one meter from the interaction region. The axes of the spectrometer, the atomic beam, and the laser beam were mutually perpendicular. A retarding potential allowed any part of the spectrum to be studied at $80 \mathrm{meV}$ resolution. A transient digitizer sampled the MCP output every $10 \mathrm{~ns}$, and a personal computer summed and stored the data. The computer could assign incoming spectra to bins based upon the energy of the laser pulse and the state (on or off) of the atomic beam.

\subsection{Apparatus}

\subsubsection{Vacuum system}

The vacuum vessel enclosed the hydrogen source, the interaction region, and the electron spectrometer. It also provided an evacuated path for the converging and diverging segments of the laser beam to prevent damage to the windows. It was pumped by a turbomolecular pump and a cryopump.

The vacuum vessel was constructed of stainless steel. It consisted of a $152 \mathrm{~mm}$ cube centered on the interaction region, a meter-long spectrometer drift tube, a six-way cross, a $305 \mathrm{~mm}$-long cylinder, a $584 \mathrm{~mm}$-long beam dump 
tube, tubes to the two pumps, ten flanges, and the housing, bellows, and tubing of the hydrogen source. Inside diameters were $152 \mathrm{~mm}$ for the cylinder, $102 \mathrm{~mm}$ for the drift tube and the cryopump tube, $73.2 \mathrm{~mm}$ for the turbopump tube, and $34.8 \mathrm{~mm}$ for the beam dump tube. The total volume of the vessel was approximately 25 liters. Three flanges were equipped with quartz windows. Three others supported parts of the electron spectrometer and one mirror inside the vacuum. The hydrogen source had one additional window for viewing the discharge.

Most of these components appear in Fig. 5, a schematic diagram of the apparatus viewed from above. The hydrogen source and the turbopump are above the plane of the figure, and the cryopump is below the plane. The atomic beam, the laser beam, and the spectrometer axis are mutually perpendicular.

The stainless steel components were joined by Conflat-type knife edge seals with copper gaskets. Teflon $\mathrm{O}$-rings were used at the windows, and a single Viton O-ring sealed a gate valve at the cryopump. The use of non-UHV materials, such as insulated wire and conductive epoxy glue, inside the vacuum was held to a minimum. The drift tube and the beam dump tube were coated with graphite (Aerodag G) to control contact potentials, reflections, and photoelectron production. The rough graphite surface is thought to have been the major source of background gases. The residual gas analyzer and two ion gauges monitored the state of the vacuum. The RGA used either a Faraday cup or an electron multiplier to detect the ion current. Its resolution was better than one atomic mass unit (amu) over the 1-200 amu range.

The vacuum system was divided into two chambers for differential pumping (Fig. 6). The upper chamber, which contained the hydrogen source, used a $350 \mathrm{l} / \mathrm{s}$ turbomolecular pump with a cold-trapped foreline. This pump held the pressure in the low $10^{-6}$ Torr range while hydrogen was flowing, and provided a background pressure of $4 \times 10^{-8}$ Torr or better. The chambers were connected by a skimmer with a $1 \mathrm{~mm}$ diameter hole which defined the atomic beam. A knife 


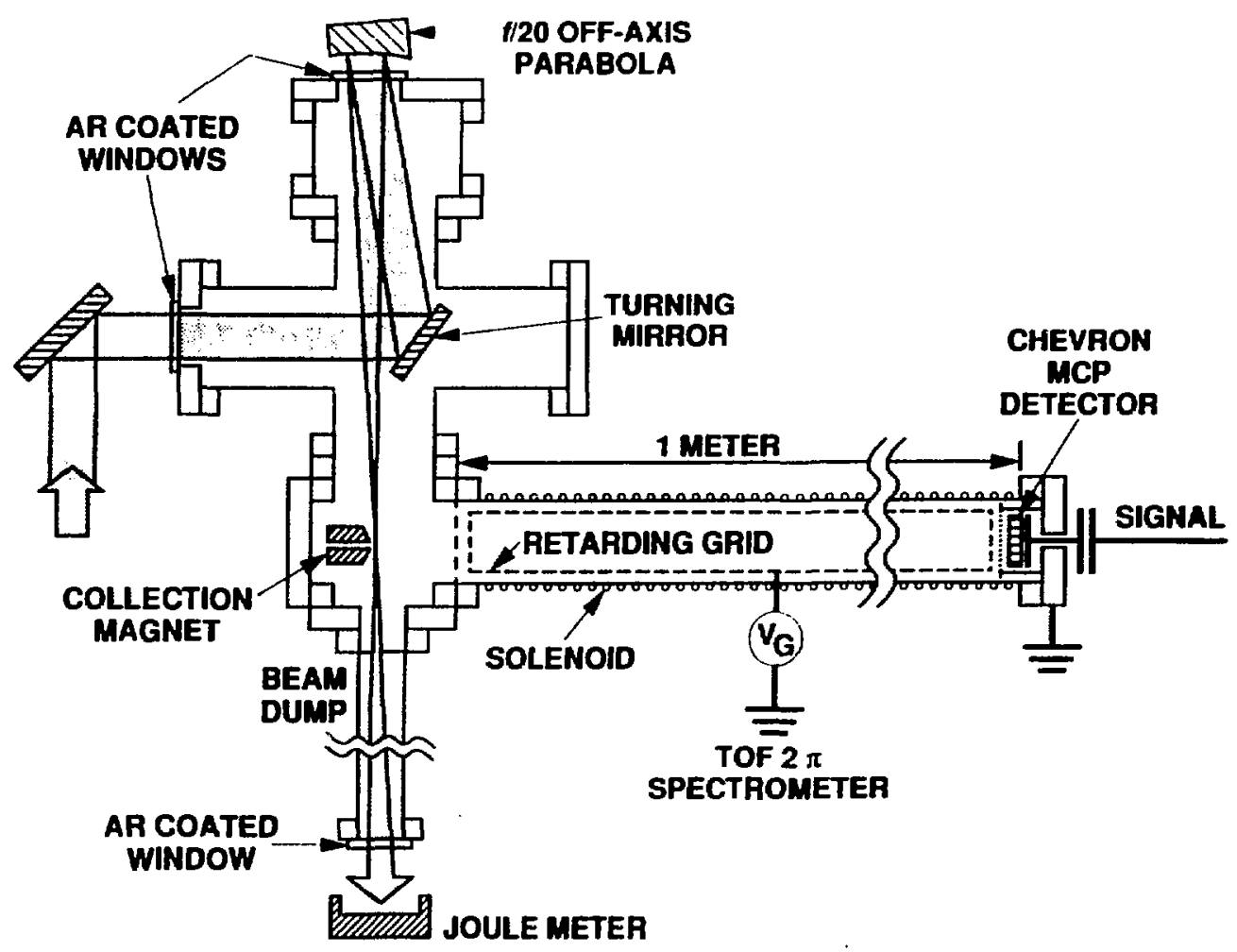

Figure 5. Schematic diagram of the experimental apparatus. The hydrogen beam points into the page.

edge around the hole prevented the gas that was retained in the upper chamber from disturbing the flow into the lower chamber. The lower chamber used an ultraclean 2000 1/s cryopump, since the gas load was not large enough to require a turbopump. The cryopump achieved a background pressure of $3 \times 10^{-9}$ Torr and $a$ working pressure in the $10^{-8}$ Torr range. Each chamber had a nude ion pressure gauge inside a $38 \mathrm{~mm}$-diameter tube attached to a $38 \mathrm{~mm}$-diameter sidearm. The RGA entrance aperture was on the axis of the $152 \mathrm{~mm}$-diameter tube leading to the cryopump, and $70 \mathrm{~mm}$ from the face of the cube. 


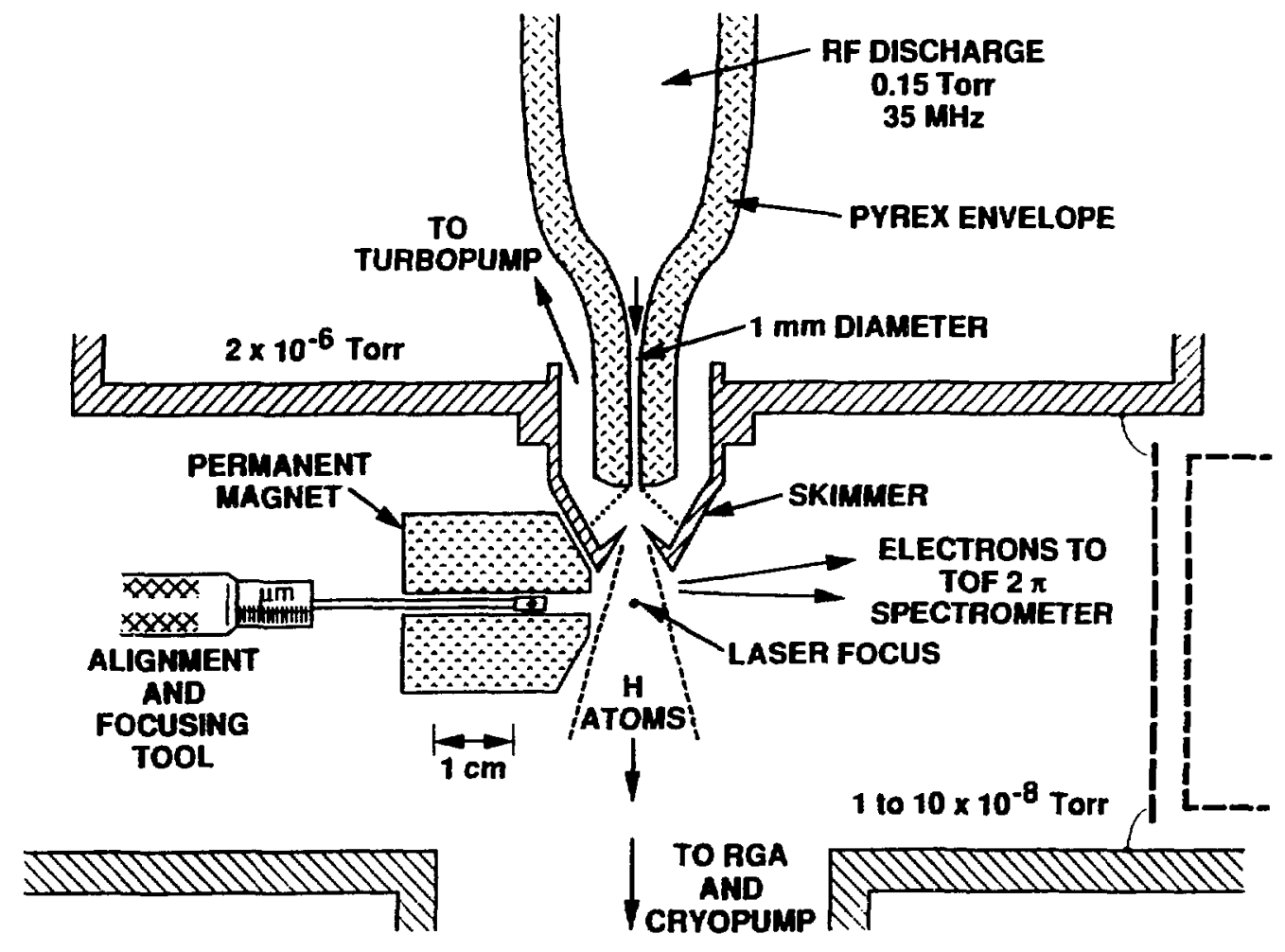

Figure 6. Schematic view of the interaction region. The laser beam comes out of the page.

The pump speeds listed above are speeds for $\mathrm{H}_{2}$ at the pump flange. The effective speeds of the pumps as used in this apparatus are $162 \mathrm{l} / \mathrm{s}$ and $960 \mathrm{l} / \mathrm{s}$ for the turbopump and the cryopump respectively. These are calculated in Appendix $B$. The connection between the cryopump and the cube was simple enough that the effective speed could be calculated from the standard formula for free molecular flow through a tube of length $L$ and diameter $D$

$$
C=12.1 \frac{D^{3}}{L+4 D / 3}
$$

where $C$ is the conductance for $\mathrm{N}_{2}$ in $1 / \mathrm{s}$, and $D$ and $L$ are in $\mathrm{cm}$. The conductance for $\mathrm{H}_{2}$ is $\sqrt{28 / 2} \mathrm{C}=3.74 \mathrm{C}$. The speed of the turbopump was derived from 
measurements of upper and lower chamber pressures as a constant gas load was divided between them. Assuming that the total gas load $Q$ was unchanged, the pressure $P_{i}$ in each chamber varied in proportion to the pump speed $S_{i}$, so that

$$
\begin{aligned}
Q=Q_{1}+Q_{2} & =S_{1} P_{1}+S_{2} P_{2}, \text { hence } \\
\frac{S_{1}}{S_{2}} & =-\frac{\Delta P_{2}}{\Delta P_{1}} .
\end{aligned}
$$

The ratio of pump speeds was found to be four.

The RGA was the most important diagnostic tool in the experiment because of the low pressures involved. In order to produce the smallest possible number of electrons, I used atomic hydrogen pressures similar to those of the background gases. The ion gauge could not measure the $\mathrm{H}_{2}$ pressure accurately, especially since ion gauges are only half as sensitive to hydrogen as to water and nitrogen, and deriving the $\mathrm{H}$ component of the total pressure when the discharge was on would require a precise value of $\mathrm{H}$ sensitivity as well. The RGA, however, measured $\mathrm{H}_{2}$ pressures independently of the others. It could also distinguish between water and nitrogen backgrounds, which occurred in varying proportions on different days and varied at different rates as the discharge warmed the upper chamber. As a bonus, the RGA served as a leak detector and verified that the vacuum was free of hydrocarbons.

\subsubsection{Atomic hydrogen source}

The atomic hydrogen source was a commercial model of the Slevin type (Slevin and Stirling 1981). It produced a discharge in a water-cooled Pyrex cavity, using up to $30 \mathrm{~W}$ of $\mathrm{rf}$ power at a nominal $35 \mathrm{MHz}$. The cavity was made to resonate at this frequency by a helical winding outside the glass. The atoms effused from a $1 \mathrm{~mm}$ diameter capillary $18 \mathrm{~mm}$ long which traversed a kink to prevent UV light from the discharge from escaping. The discharge required a gas pressure of at least $4 \times 10^{-8}$ Torr, so the pressure at the outlet of the capillary 
was typically $2 \times 10^{-6}$ Torr. The rf power could be gated off by an external $5 \mathrm{~V}$ signal to modulate the $\mathrm{H}$ signal.

Commercially pure hydrogen flowed through a sapphire leak valve, and then through a heated palladium filter, which further purified the hydrogen and controlled the pressure in the discharge. The palladium filter could be bypassed in order to admit krypton or other gases to the lower chamber. The sapphire valve provided very good control of the pressure in this case.

Heat from the discharge caused the resonant frequency of the cavity to increase. The original tuning range of the if power supply was not large enough to follow the resonance. I solved the problem by changing the relative sizes of fixed and variable resistors in the frequency control circuit, thereby doubling the tuning range from $\pm 0.2 \mathrm{MHz}$ to $\pm 0.4 \mathrm{MHz}$ about a center frequency of $37.2 \mathrm{MHz}$.

The major product of the discharge process was, of course, atomic hydrogen. I calculated the dissociation fraction from the change in the $\mathrm{H}_{2}$ pressure at the RGA. There are two common definitions of dissociation fraction (Chan et al. 1988). The one used here is the fraction of the total number of atoms and molecules that are atoms:

$$
F=\frac{\rho_{1}}{\rho_{1}+\rho_{2}}
$$

where $\rho_{1}=$ number density of $H$, and $\rho_{2}=$ number density of $H_{2}$ when the discharge is on. When each atom and each molecule is once ionized, $F$ is the fraction of electrons that come from atoms. The alternate definition is

$$
F^{\prime}=\frac{\rho_{1}}{\rho_{1}+2 \rho_{2}} \text {. }
$$

This is the number of dissociated molecules divided by the number of molecules initially present.

The measured pressure at mass two $P_{2}$ is related to gas density according to

$$
P_{2}=\alpha_{22} \rho_{2}+\sum_{i \neq 2} \alpha_{2 i} \rho_{i}
$$


where $\alpha_{22}$ is the mass two response to $H_{2}$ molecules and $\alpha_{2 i}$ is the mass two response to atoms or molecules of mass $i$. All of the $\alpha_{2 i}$ except $\alpha_{22}$ were taken to be zero, since there was no evidence of such responses to other gases in the system, and none was expected. (The mass one readings are affected by $\rho_{2}$, however. See Appendix B.)

When the discharge started, the measured $\mathrm{H}_{2}$ pressure dropped from $P_{2}^{\text {off }}$ to $P_{2}^{o n}$. For every molecule dissociated, two atoms were formed. The dissociation fraction was therefore

$$
F=\frac{2\left(P_{2}^{\mathrm{off}}-P_{2}^{\mathrm{on}}\right)}{2 P_{2}^{\mathrm{off}}-P_{2}^{\mathrm{on}}}
$$

Typical readings when the end of the capillary was $5 \mathrm{~mm}$ above the skimmer were $P_{2}^{\text {off }}=1.3 \times 10^{-7}$ Torr, $P_{2}^{\text {on }}=9 \times 10^{-8}$ Torr, indicating $F=47 \%$. When the capillary was $27 \mathrm{~mm}$ above the skimmer, typical readings were $P_{2}^{\text {off }}=4.2 \times$ $10^{-8}$ Torr, $P_{2}^{\text {on }}=3.3 \times 10^{-8}$ Torr, indicating $F=35 \%$. These values of $F$ would be higher if $\rho_{1}$ and $\rho_{2}$ were adjusted to account for recombination in the lower chamber.

\subsubsection{Optical system}

\subsubsection{LABS-I laser}

During the present experiment, the LABS-I laser system remained essentially as it was in 1988 (Roberts et al. 1988). A linearly polarized seed pulse at $248 \mathrm{~nm}$ wavelength ( $5.0 \mathrm{eV}$ photon energy) was amplified by two $\mathrm{KrF}$ amplifiers. Seed pulses were produced at a $5 \mathrm{~Hz}$ rate. The amplifiers could be operated at $5 \mathrm{~Hz}$ or its submultiples. The average length of a pulse has been measured to be $600 \pm 50$ fsec FWHM, using two photon ionization of NO. Observations with a streak camera have verified that the energy was concentrated into a single short pulse when the "front end" was timed correctly. The measured pulse length was close enough to the transform limit to justify modeling the pulse envelope as a smooth rise and fall with no subpeaks. The final pulse energy from LABS-I 
reached $30 \mathrm{~mJ}$ or more when the timing was perfect and the gas was fresh. At optimal adjustment, the energies of the individual pulses had a standard deviation equal to $3 \%$ of the mean. As the times of the discharges in the $\mathrm{KrF}$ amplifiers drifted away from the arrival of the seed pulse, the average energy decreased, and the pulse to pulse variation increased.

Sensitivity to timing was part of a tradeoff in which the seed pulse was placed early in the KrF amplifiers' gain window to minimize the effects of amplified spontaneous emission (ASE). ASE occurred throughout the discharge because of the high gain of $\mathrm{KrF}$, but it had little effect on multiphoton ionization for two reasons. One was that the amplifiers used no mirrors, so their ASE diverged strongly, whereas the seed pulse was collimated. A simple aperture could therefore block most of the ASE that was produced. The other reason was that the total ASE energy, which was small to begin with, was spread over a $20 \mathrm{nsec}$ period. The resulting irradiance was too low to drive a three photon process, as confirmed by blocking the seed pulse during data collection.

There were three significant changes to the system during the experiment. One was replacement of several front-end amplifiers. This had no known effect on my experiment. The second was improvement of the discharge in the first $\mathrm{KrF}$ amplifier, which allowed the second $\mathrm{KrF}$ amplifier to operate at lower gain with a different gas mixture. This reduced the level of ASE and probably improved the uniformity of the beam, but it did not change the electron spectra. The third change was insertion of a saturable absorber in the dye amplifie: ciain to reduce the level of ASE from these amplifiers. This increased the shot-to-shot variation in total UV energy, but it had little or no effect on the electron spectra.

\subsubsection{Windows, mirrors, and beamsplitters}

All of the optical components were made of Corning 7940 fused silica. This was important even for "max $R$ " mirrors, those made with the highest possible reflectivity of the design wavelength, since $0.2 \%$ of the UV beam was still a 
high power level. Reflecting surfaces were formed with dielectric coatings for accurate reflectance and low absorption, or used uncoated for $10 \%$ reflection of S-polarized light at a $45^{\circ}$ angle of incidence. Other surfaces were anti-reflection coated, including both sides of all windows, but not including the backs of "max $R$ " mirrors. Surface figures were $\lambda / 10$ at $633 \mathrm{~nm}$, which is $\lambda / 4$ at $248 \mathrm{~nm}$. Actual reflectances of the beam splitters were measured at the angles of incidence used, either $45^{\circ}$ or $50^{\circ}$. These measurements were performed using the full power of the laser in case the reflectances varied with irradiance. No such variation was found.

\subsubsection{Photodiode and joulemeter}

A photodiode provided a trigger signal to the data collection electronics. In some cases, it observed the UV that passed through a "max $R$ " mirror. Other times, it sat next to the beam and picked up UV scattered from the air. Since the trigger signal stopped when the beam was blocked or the laser misfired, null spectra were not averaged with the rest. The photodiode was fast enough that the picosecond pulse appeared as a $2 \mathrm{~ns}$ excursion above the $20 \mathrm{~ns}$ ASE pedestal. This was too short for reliable triggering, so the actual trigger generally was based on ASE. This caused a minor problem on days when the ASE was not tightly locked to the picosecond pulse by adding one or two time channels to the widths of the peaks in the output of the transient recorder. The spread was no more than this because the total duration of the ASE was only two time channels. The minor problem was that it was harder to recognize small ATI peaks when they were spread over more time channels. The error in measured electron energy due to a time error of one channel, ranging from $11 \mathrm{meV}$ for $1 \mathrm{eV}$ electrons to $346 \mathrm{mev}$ for $10 \mathrm{eV}$ electrons, was not a problem, since any peak could be retarded to $1 \mathrm{eV}$ energy for close study.

A Gentec ED-500 joulemeter provided another trigger signal, and also the energy of each laser pulse, by measuring the energy passing through the first 
beamsplitter. A Gentec PRJ-M readout corrected for shifts in the baseline signal, applied the calibration factor of $2.2 \mathrm{~V} / \mathrm{J}$, and delivered its output directly to the lab computer. I checked the joulemeter's calibration against a new ED-500 that was calibrated at $2.4 \mathrm{~V} / \mathrm{J}$. The meters agreed within the implied uncertainty of $0.05 \mathrm{~V} / \mathrm{J}$. I could not test the linearity of the ED-500 in its normal operating range with neutral density filters because the unattenuated beam damaged the filters. Instead, I checked the linearity of an ED-100 joulemeter, which works the same way but produces more volts per joule, using attenuated picosecond pulses. Its linearity was good, so there is no reason to doubt the linearity of the ED-500. In addition, data sets which have been divided into bins according to ED-500 readings show a linear relation between bin number and the area of the scattered light peak, as they should.

\subsubsection{Mechanical Support}

The vacuum chamber and the optical system, other than the LABS-I laser, the joulemeter, and the photodiode, resided on a 2 inch thick optical breadboard. The focusing mirror sat on a motor-driven three-way translation stage, which sat on a half-inch steel plate cantilevered off the end of the breadboard. The mirror inside the vacuum was mounted to a flange on the cross. The other mirrors were mounted on vibration-damping posts fixed to the table. The vacuum chamber supported the two pumps. The breadboard was supported by a large table on the drift tube side, and by two rigid legs to the floor on the other side. These tables were not isolated from vibrations in the floor because the optical system had to be fixed in position to remain aligned with the UV beam.

\subsubsection{Electron time-of-flight spectrometer}

Electrons generated at the laser focus were collected and their energy was analyzed using an electron time-of-flight spectrometer with $2 \pi$ sr solid angle of collection. The spectrometer was based on the Kruit and Read design (Kruit and 
Read 1983) as adapted at Chicago (Luk and Rhodes 1988), using a permanent magnet rather than an electromagnet. The field of the permanent magnet merged with that of a solenoid wourd around the drift tube. A retarding potential could be placed on a Faraday cage around the electron drift region to increase the flight time, thus improving the resolution, and to discriminate against low energy electrons. The electrons were detected by a two-stage chevron microchannel plate electron multiplier. The drift direction was perpendicular to the axes of the hydrogen beam and the laser beam.

\subsubsection{Theory of operation}

The purpose of the magnetic field in this kind of spectrometer is to capture all of the electrons emitted into the hemisphere facing the detector and bring each one to the detector at a time representing its original speed regardless of its initial direction. This process, described by Kruit and Read, basically uses conservation of angular momentum to convert cyclotron motion about the field lines into linear motion along the drift tube without changing the electron's energy. When the initial magnetic field diverges rapidly, the electron trajectories become parallel in a time short compared to the drift time. This minimizes the spread of arrival times for electrons of equal energy but different initial directions of motion.

The angle between an electron's initial velocity and the axis of the spectrometer is $\theta_{i}$ in the notation of Kruit and Read. At a later time, the new angle $\theta$ is determined by the local magnetic field $B$ and the initial magnetic field $B_{i}$ as

$$
\frac{\sin \theta}{\sin \theta_{i}}=\left(\frac{B}{B_{i}}\right)^{1 / 2} .
$$

The distance $z_{p}$ required to reduce $\sin \theta$ by a factor of ten may be estimated from measurements of the field of a near-twin of the magnet in the spectrometer. $\mathrm{My}$ measurements of $B$ along the axis may be modeled as

$$
B(z)=\frac{\left(B_{i}-B_{\infty}\right)}{\left(1+(z / a)^{b}\right)^{c}}+B_{\infty},
$$


where $z$ is distance from the interaction region, $B_{i}=0.27 \mathrm{~T}, B_{\infty}=-3.5 \times 10^{-4} \mathrm{~T}$, $a=10.0 \mathrm{~mm}, b=1.54$, and $c=1.455$. This value of $B_{\infty}$ is apparently the error in setting the zero reading of the gaussmeter. Then $B\left(z_{p}\right)=0.01 B_{i}$ when $z_{p}=24 \mathrm{~mm}$, or $2.2 \%$ of the total drift distance.

Kruit and Read define two measures of adiabaticity to judge whether the approximations in Eq. 29 are valid. One, termed $\chi_{1}$, is the fractional change in $B$ during one cyclotron orbit. The other, $\chi_{2}$, is the angle through which the image rotates before reaching the detector. In their spectrometer, the maximum value of $\chi_{1}$ was $0.78 E_{K}^{1 / 2}$, where $E_{K}$ is the electron energy in $\mathrm{eV}$, and the value of $\chi_{2}$ was $0.063 E_{K}^{1 / 2}$. These were small enough to justify the approximations. In our spectrometer, the largest value of $\chi_{1}$ was $0.063 E_{K}^{1 / 2}$, and the value of $\chi_{2}$ was $0.035 E_{K}^{1 / 2}$. These smaller values are primarily due to our large value of $a$, compared to Kruit and Read's $3 \mathrm{~mm}$.

Within each peak, the first electron to arrive is that electron which traveled along the line of sight between the focus and the MCP detector. The electrons that leave at other angles travel farther than the measured distance from the laser focus to the detector and appear to be less energetic. Electrons may also be delayed by space charge. Thus we label the peaks by the highest energy component of the peak rather than by the energy of the average electron. The shape of the peak may be significant, however, when electrons formed in different parts of the focal volume arrive with different energy shifts from the ponderomotive potential. This is discussed in Chapter 4.

Since each electron follows a field line diverging from the interaction region, the spectrometer produces a magnified image of the interaction region on the detector. The image has a blur circle, similar to the Airy distribution in optical images, due to the cyclotron motion about the field line. This imaging process spreads the electrons over the surface of the detector, reducing the chance of encountering a channel whose charge has been depleted by a faster electron. The 
magnification factor is $\sqrt{B_{i} / B_{f}}=9.1$, where $B_{f}$ is the $0.033 \mathrm{~T}$ field at the detector. The image of the interaction region is roughly $0.6 \mathrm{~mm}$ by $12 \mathrm{~mm}$, depending on what is considered to be the edge of the interaction region. The number of channels in a rectangle this size is $\mathbf{4 6} \mathbf{0 0 0}$. Only at the highest irradiances was the number of detected electrons comparable to this, and in those cases the effective radius of the interaction region was probably much larger.

A simple formula connects an electrons's fight time $T_{F}$ to its kinetic energy $E_{K}$ :

$$
E_{K}=\left(\frac{F L_{D}}{T_{F}}\right)^{2}
$$

where $F=\sqrt{m / 2}=1.68 \mathrm{eV}^{1 / 2} \mathrm{sec} \mathrm{m}{ }^{-1}, m$ is the electron mass, and $L_{D}=$ $1.086 \mathrm{~m}$ is the drift length. Electrons of 1 to $10 \mathrm{eV}$ energy leave the interaction region with enough speed that an extraction field is not needed, but not with so much that relativistic corrections are important. A representative speed is $2 \mathrm{~m} / \mu \mathrm{sec}$, or $0.8 \%$ of the speed of light. The relation between units of time and units of energy is described by

$$
\frac{d T_{F}}{d E_{K}}=-\frac{F L_{D}}{2 E_{K}^{3 / 2}}=\frac{-T_{F}^{3}}{2 F^{2} L_{D}^{2}} .
$$

These formulae are complicated slightly when a retarding potential is used, because the drift speed is different before and after the grids. In this case, table lookup is easier than formula evaluation. The small distance between the two grids permits details of the acceleration to be neglected.

\subsubsection{Construction}

Figure 5 shows a cross section of the permanent magnet in a plane including the magnet's axis, which was also the spectrometer's axis. Because of the beveled edge and the axial hole, the front part of the magnet acted as a pole piece. The magnet I tested produced a maximum field of $0.33 \mathrm{~T}$ in a ring of radius $4.5 \mathrm{~mm}$ about the axis and $3 \mathrm{~mm}$ from the face of the magnet. The field was $0.27 \pm 0.01 \mathrm{~T}$ 
throughout a region larger than the focal volume of the laser. In the plane of the ring, which included the longest dimension of the focal volume, the magnetic field increased from $0.27 \mathrm{~T}$ on the axis to $0.28 \mathrm{~T} 1 \mathrm{~mm}$ from the axis. The field measured on the axis $1 \mathrm{~mm}$ either direction from the plane of the ring was $0.26 \mathrm{~T}$. The magnet used in the spectrometer produced the same peak field onaxis, but at a point $4 \mathrm{~mm}$ from the face of the magnet. Kruit and Read obtained a stronger and more strongly diverging field with their water cooled electromagnet and customized pole piece, so their energy resolution was better.

A pair of grids at the entrance to the drift tube applied the retarding potential over a distance of $3 \mathrm{~mm}$. The first grid was connected to the cube, so that the interaction region was not affected. The second grid was part of the Faraday cage which minimized electrical steering of the drifting electrons. The grids were made from electroformed copper of $90 \%$ transparency.

In the original construction, the copper grids were coated with colloidal graphite, as the rest of the cage was, to address the problems of contact potentials, UV reflection, and photoelectron production. Contact potentials between different materials in the drift iube can distort the electron trajectories, with different distortions at different places in the tube. This increases the variation in times of arrival at the detector, and it may add a constant potential to the applied retarding voltage. Coating the surfaces with a conducting film reduces the forces felt by the drifting electrons. Contact potentials did not offset the applied voltages very much, as evidenced by good agreement among electron spectra collected with different retarding voltages. Comparisons between spectra taken months apart showed shirts of tens of $\mathrm{meV}$ in peak positions, however.

The rough surface of the graphite, which appears black to the eye, was effective in preventing UV reflections, which could produce photoelectrons of various energies at many surfaces in the apparatus and add to the background in the spectra. Photoelectron production was not completely eliminated, however. 
The photon energy of $5 \mathrm{eV}$ is greater than the work function of any material, so the graphite itself emitted photoelectrons. Still, with a maximum energy of $0.2 \mathrm{eV}$, they were much less of a problem than those that would come from a steel surface. A new set of grids with gold flashing was tried, in hopes that their work function would be higher, but the substitution made no difference in the electron spectra.

In order that the magnetic field in the drift tube run parallel to the axis, I designed a set of Helmholtz coils to cancel the terrestrial field inside the tube. Otherwise, electrons that reach the detector must start off-center in the interaction region and graze the wall at the entrance to the drift tube. The Helmholtz coils were rectangular, of width $23 \mathrm{~cm}$ and of a length considerably greater than the drift region, separated by $18 \mathrm{~cm}$. These dimensions produced a very uniform field through the $2 \mathrm{~cm}$ diameter region of electron current, with variations less than $0.5 \%$ of the $4.2 \times 10^{-5} \mathrm{~T}$ on-axis field. Each coil consisted of two turns of wire and carried 2.4 A. A high-current design was selected so that currents induced in the coils when the laser fired would be a small perturbation. The field to be canceled was vertical for a considerable distance above the steel table, so one pair of Helmholtz coils was sufficient.

\subsubsection{Signal processing and data storage system}

This system consisted of an electron detector, several devices to provide measurements in digital form, and a computer to control the other devices and store the data. The following sections describe these components, including the data collection programs and digital interfaces that make the system work.

\subsubsection{Microchannel plate electron detector}

A microchannel plate assembly at the end of the drift tube detected and amplified the electron current. It also provided a "time zero" signal from scattered light as the picosecond laser pulse passed. MCP's have many desirable properties 
for this application. They detect both electrons and UV photons, thus marking both the beginning and the end of the drift period. They produce a sharp pulse (2 ns FWHM) from a single electron. Chevron MCP's such as the ones used here are capable of gains of $10^{7}$ with low rates of feedback from acceleration of ions in the backward direction. Their gain is easily adjusted to provide a signal of convenient size without further amplification. The time required to recharge after an output pulse, several milliseconds, is much less than the time between laser pulses. The charge available from each channel is independent of all others except its nearest neighbors, so all electrons are counted equally if they are spread thiniy enough over the surface. An accelerating potential of $250 \mathrm{~V}$ brings the electrons to the first plate with the optimum energy for detection regardless of their drift energies, and also recaptures many electrons that strike the glass matrix between channels. The detection efficiency for electrons is therefore around $70 \%$ (Fraser 1983). The detection efficiency for $248 \mathrm{~nm}$ photons is approximately $4 \times 10^{-9}$ (Wiza 1979).

A disadvantage of MCP detectors is the statistical nature of electron multiplication. Multiplication occurs only when electrons strike the wall of a channel. Secondary electrons from each encounter with the wall fly off in many directions, some of which lead to many more collisions and others to very few. The probability of producing an output pulse of given total charge therefore decreases exponentially with the amount of charge. This makes it difficult to calculate the number of incident electrons that produced a particular peak in the electron spectrum. In some MCP's, this exponential distribution can be converted to one which is roughly Gaussian by increasing the applied voltage. This causes depletion of the charge in the channel walls when a large swarm of electrons passes. Further multiplication of that swarm is reduced. A peak appears in the distribution at the limiting electron density, and there is an average gain that is relatively insensitive to variations in the applied voltage. In the present experiment, the 
distribution did not develop a peak even at the highest recommended voltage of $1000 \mathrm{~V}$ per plate. Measurements of peak heights for single-electron events showed an exponential falloff in the low range, then a region of slowly-varying probability. Moreover, this is not the distribution that was finally recorded. The LeCroy TR8818 transient digitizer had its own statistical distribution of responses to the MCP output pulses, which were shorter than the 10 nsec sampling period of the digitizer. The final result was again a decreasing exponential over the range of identifiable responses. An average recorded voltage of $7 \mathrm{mV}$ per incident electron, when $2.209 \mathrm{kV}$ was applied to the resistor chain, has been derived, but this value depends upon the model for how many incident electrons were not recorded at all. Details of these measurements appear in Appendix D.

\subsubsection{CAMAC and GPIB devices}

The instruments used to measure the laser pulse energy and the MCP output from every laser pulse were connected to the lab computer by CAMAC (Computer Automated Measurement And Control, IEEE Standard 583-1975) and GPIB (General Purpose Interface Bus, IEEE Standard 488-1978) interfaces. These interfaces allowed a program written in Microsoft QuickBASIC 3.0 to control the instruments, read the instrument status, and read the measured values rapidly enough to keep up with a $2.5 \mathrm{~Hz}$ repetition rate. Another GPIB link allowed the program to collect electron spectra with the discharge alternately on and off by reading the status of a delay generator which inhibited the discharge. Figure 7 presents a block diagram of the CAMAC and GPIB devices.

The data collection programs, named ERUN (Energy RUN) and DRUN (Double spectra RUN), called subroutines from a software package that came with the National Instruments GPIB controller board. These subroutines sent commands either to the controller board or through it to the GPIB devices. Some commands caused the devices to return measured data or information about their internal status across the GPIB. Others, sent to the LeCroy 8701A CAMAC to 


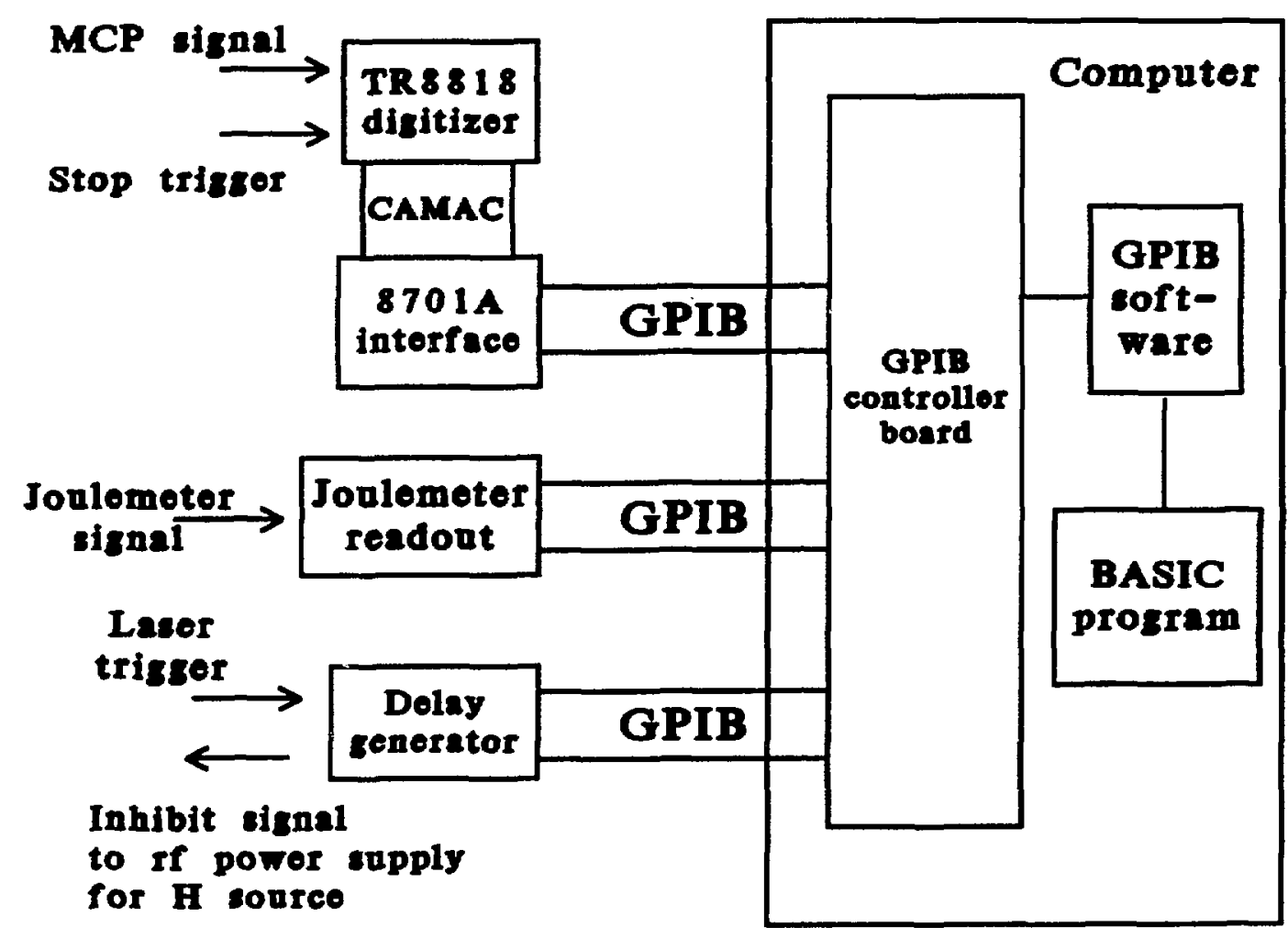

Figure 7. Block diagram of CAMAC and GPIB devices.

GPIB interface, caused the $8701 \mathrm{~A}$ to send CAMAC commands to the TR8818 transient recorder. These CAMAC commands might then cause the TR8818 to collect spectral data or to report the data or its device status to the $8701 \mathrm{~A}$. In the latter case, the $8701 \mathrm{~A}$ transferred the information to the GPIB.

The three TR8818 transient recorders, also referred to as digitizers, that have been used at different times all worked as advertised on the CAMAC side. 
The BASIC program set parameters such as sample rate and range of voltages to be measured, then started the TR8818 digitizing according to its irternal clock. Measured voltages were stored in a LeCroy 8103A memory module in wrap-around fashion so that the latest 1000 values were always available. The measurement stopped when a trigger signal arrived from the vacuum photodiode. A Stanford Research DG535 delay generator delayed the trigger until the slowest electrons had arrived at the MCP. The TR8818 then set a Look-At-Me (LAM) flag. A single pair of BASIC calls then commanded the TR8818 and the 8701A to transfer data to an array in the BASIC program until the array was filled.

The PRJ-M joulemeter readout was equipped with GPIB output connections, though it did not operate fully within the GPIB standard. It reported a triggered condition by asserting a service request (SRQ) on the GPIB.

\subsubsection{Data collection programs}

At the start of this work, there was a BASIC program called TR88QPG1, later renamed RUN, that operated the TR8818 and stored averaged electron spectra on disk. It could also print or plot data on the computer monitor or on paper. RUN incorporated an assembler language subroutine to sort out the TR8818 measurements, which the computer received in compressed form. RUN had one significant problem, however. It would "hang up" from time to time, waiting for a LAM that never came. I wrote an improved version, called NEWRUN, which ran without hangups and was easier to use. Also, NEWRUN's assembler subroutine was $50 \%$ faster than the original. Listings of RUN, NEWRUN, and their successors ERUN and DRUN appear in Appendix E.

In order to measure the change in ionization rate with irradiance, one needs more than the averaged spectra from RUN. The average energy of a laser pulse changed quite rapidly at times, often several percent per minute. In addition, the pulse to pulse variation was amplified by the $I^{N}$ dependence of the ionization rate. The ERUN program provided more information by averaging each new 
spectrum with others corresponding to the same laser pulse energy. The program maintained 14 bins of equal width in pulse energy. The bin width was set to $3 \%$ of the mean pulse energy at the time the program started. Guard bins counted the pulses falling above and below the data bins.

An ERUN data file thus contains a progression of spectra representing contemporaneous conditions of the laser, the target gas, the measurement electronics, and all other factors. Ideally, the only difference among the spectra is the irradiance in the interaction region. Practically, there is no way to determine whether changes in the laser's pulse width or wavefront distortion were correlated with pulse energy, or whether these parameters change from time to time independently of pulse energy. Also, when the average pulse energy drifted, spectra in the high-energy bins were not contemporaneous with those in the low-energy bins. These concerns were addressed by storing data every ten minutes or so and comparing sets that should be the same. A good match is not proof that the irradiance was the only thing changing, and it says nothing about correlations, but it is the best indicator available.

In addition to acquiring and processing data, the ERUN program coordinated the joulemeter and the digitizer with the laser and with each other. Both devices were triggered by the laser pulse, but independently. The PRJ-M triggered internally on the joulemeter signal, while the TR8818 received its trigger from a photodiode. Either trigger could occur without the other. ERUN had to verify that both devices have been triggered by a single laser pulse. It started by performing a read and clear operation on the PRJ-M, then waiting for the next SRQ before starting the TR8818 for the first time. This synchronized the program to the laser. From then on, ERUN could clear the PRJ-M, then start the TR8818, then return to wait for the PRJ-M to assert SRQ before the next laser pulse arrives. If the SRQ was late, ERUN displayed a warning on the monitor and started a new collection cycle in case the TR8818 received its trigger. When the SRQ 
occurred, ERUN checked that the TR8818 had set its LAM, indicating its trigger came from the same laser pulse. All of this clearing and checking, together with reading and processing the electron spectra, required about $200 \mathrm{~ms}$. In some cases, ERUN kept up with the laser at its maximum $5 \mathrm{~Hz}$ repetition frequency, but sometimes there was no increase in signals from bin to bin, indicating there was no correlation between the energy of the pulse and the bin to which the data were assigned. The final collections were conducted at $2.5 \mathrm{~Hz}$ to allow a safety margin.

A variation of the ERUN program, called DRUN, was sometimes used to obtain contemporaneous spectra with and without atomic hydrogen targets. In these collections, a second DG535 delay generator sent an inhibit signal to the if power supply that drove the discharge in the hydrogen source. The delay was set to stop the discharge for a long enough time that all of the atomic hydrogen recombined or was pumped away, about one second. During this time, trigger pulses continued to arrive from the photodiode. The status word from the DG535 showed both whether it was conducting a timing cycle at the time it was polled, and whether the latest trigger arrived before or after the timing cycle started. DRUN could therefore identify the incoming spectra that resulted from the laser pulse that turned off the discharge and those from the last pulse before the discharge started again. Eight energy bins were used for spectra with atomic hydrogen present, and the other eight for atom-free spectra. Spectra from intermediate pulses were discarded. Since only eight bins were used for each set of spectra, the bin width was usually set to $5 \%$ of the mean pulse energy, rather than $3 \%$.

Another variation on ERUN was intended to count electrons instead of measuring current, but this approach did not work well with the mixture of large and small pulses from the MCP. For this variation, I modified the subroutine that unpacked data from the TR8818 to add one unit to the destination array when the measured value exceeded a threshold, rather than adding the measured 
value. The subroutine then simulated a discriminator. Unfortunately, it counted echoes of MCP pulses, which resulted from imperfect impedance matching, the same as the original pulses. These echoes were not a problem when the original program summed the measured voltages, because they were accounted for in the calibration process. Raising the discrimination threshold enough to block the echoes of the largest pulses reduced the probability of detecting real elecirons by a large factor that could not be determined accurately, and which was quite sensitive to the value of MCP gain. In addition, a discriminator cannot distinguish between single and multiple electron events, while a noisy current measurement can, if averaged over enough samples.

Special techniques were sometimes necessary for controlling the CAMAC and GPIB devices. For example, the RUN program failed sporadically because it inadvertently caused the $8701 \mathrm{~A}$ to reissue the TR8818 start command before it tested for a LAM. Whenever the second start command arrived after the stop trigger, the TR8818 wrote over the stored data and then rejected the readout command when it arrived. The root of the problem lay in the $8701 \mathrm{~A}$ command structure. The procedure for sending a CAMAC command is to load the command into a buffer in the $8701 \mathrm{~A}$, then to send a "talk" command over the GPIB. The 8701A then talks on both the GPIB and CAMAC circuits. There is no difference between a "talk" command that is intended to send a CAMAC instruction and one that is not. This means that a request for device status, for example, causes the $8701 \mathrm{~A}$ to send some command to the TR8818 as it sends its status byte to the GPIB controller. The BASIC programmer must ensure that the CAMAC command in the 8701A's buffer is harmless. Similarly, when testing the TR8818's status after a CAMAC command, the programmer must avoid resetting the status byte before reading it.

The GPIB link to the Gentec PRJ-M joulemeter readout also required special attention. When it placed a reading into the output buffer, it asserted a 
Service Request (SRQ), which is typical of GPIB devices. However, if the GPIB controller was slow to read the buffer, the SRQ would be deasserted. When this happened, the unread output remained in the buffer until it was read. There was no command to clear the buffer. ERUN therefore started each collection cycle by reading from the PRJ-M until it was clear. Then, once the program began waiting for the PRJ-M's SRQ, it could do nothing else. Another peculiarity of the particular unit used was that the leading digit of the measured energy was sometimes omitted from the GPIB output, though it appeared on the display. This was accommodated by attenuating the laser pulse or adjusting the readout's sensitivity so that the leading digit was always zero. The dynamic range of the readings was still larger than that of the ERUN program.

\subsubsection{Alignment of the interacting beams}

The laser focus had to overlap the atomic beam in a well-known way to permit calculation of an electron production rate from theoretical cross sections. In addition, the interaction had to occur at the position of maximum magnetic field for the electron spectrometer to work properly. Fortunately, the design of the apparatus allowed enough tolerance in these conditions that they could be met reliably. Alignment tolerance is increased when one of the entities (beams or fields) to be crossed is much larger than the other. Either can then be shifted by distances comparable to the smaller dimension without greatly affecting conditions in the overlap region. In this case, the focal region of the laser was larger than the atomic beam along the axis of the focus, but much smaller in the radial direction, and the characteristic dimensions of the magnetic field were larger than any of the others. Values of the characteristic dimensions are as follows: the radius of the atomic beam varied from 1.2 to $1.7 \mathrm{~mm}$, depending on the height of the hydrogen source. The irradiance contour at half the peak irradrance was approximately $21 \mu \mathrm{m}$ in radius and $5 \mathrm{~mm}$ in length. The magnetic field changed 
by about $4 \%$ per millimeter. Thus the alignment tolerances in the interaction region were large fractions of a millimeter, and no unusual care was required in the alignment process. Further, motion of the focal volume by tens of micrometers during data collection did not affect the data.

The first step in the alignment process was to place the axis of the hydrogen beam and the point of maximum magnetic field at the center of the cube, using an alignment telescope with $25 \mu \mathrm{m}$ (0.001 in.) resolution and crosshairs placed on the cube's vacuum flanges. Translation micrometers on the hydrogen source were used to place the capillary directly over the center, and the vertical translation micrometer was exercised to verify that its motion was purely vertical. A $0.2 \mathrm{~mm}$ adjustment was made to bring the capillary over the hole in the skimmer. The magnet was placed on the cube axis with its face $4 \mathrm{~mm}$ from the center of the cube. Then a $50 \mu \mathrm{m}$ diameter pinhole mounted on a micrometer vacuum feedthrough was placed as near the center as possible, and the micrometer reading was recorded. The error in placing the pinhole was $0.4 \mathrm{~mm}$.

Next, an alignment reference was established. A spatially filtered HeNe laser on the table with the vacuum chamber provided a collimated, visible beam. The red beam was brought into the vacuum chamber along an axis of the cross, then reflected from a mirror inside the cross to meet the center of the window at the paraboloid mirror. The paraboloid mirror had previously been oriented with its axis along the direction now taken by the red beam. Translation of the paraboloid then placed its focus at the pinhole at the center of the cube. After this, the UV beam could be tested against the red beam.

An error budget assured that aberrations caused by misalignment of the laser beam to the paraboloid axis would be less than the diffraction limit. Since the diffraction limit at $f / 20$ was relatively large, this permitted a 90 mrad error. Errors in the positions set by alignment ielescope and by diffraction from crosshairs were in the microradian range, so a very generous budget remained 
for determining the paraboloid's axis and locating the center of its window. The fact that the focus was not diffraction limited made the optical alignment ever less of a concern.

The final alignment step was repeated before each period of data collection: the focusing mirror was translated along all three axes to maximize the production of ATI electrons. This accounted for flexing of the table with changing temperature, possible loss of position of the motor micrometer stages, changes in collimation of the UV beam, and unknown variables. In doing this, I found that the spectra were little changed by moving the focus $2 \mathrm{~mm}$ either way along the laser beam axis, since the focal region was very long. ATI signals, nearly absent when the spot was near the magnet, increased rapidly over a distance of $200 \mu \mathrm{m}$, then remained steady as the spot moved away from the maximum field position. The tolerance for motion along the atomic beam was $300 \mu \mathrm{m}$ either way. Figure 8 shows the changes in the height of the $S_{0}$ peak of $H$ with mirror translation.

\subsection{Calibration}

\subsubsection{Irradiance at focus}

I measured the irradiance at focus in two independent ways. One was to scan the focal spot across a pinhole to map out the shape and size of the irradiance distribution function in three dimensions. The other was to examine the distribution of electron energies within an ATI peak to determine the maximum ponderomotive shift, which is proportional to the highest irradiance experienced by the last atom to ionize. The pinhole measurements are described in Appendix $\mathrm{C}$ and summarized here. The energy shift measurements appear in Chapter 5.

I measured the average spot size at the $f / 20$ focus with a $25 \mu \mathrm{m}$ diameter pinhole and a pair of vacuum photodiodes. One diode measured the incident pulse energy, using part of the energy that had to be rejected to avoid enlarging 

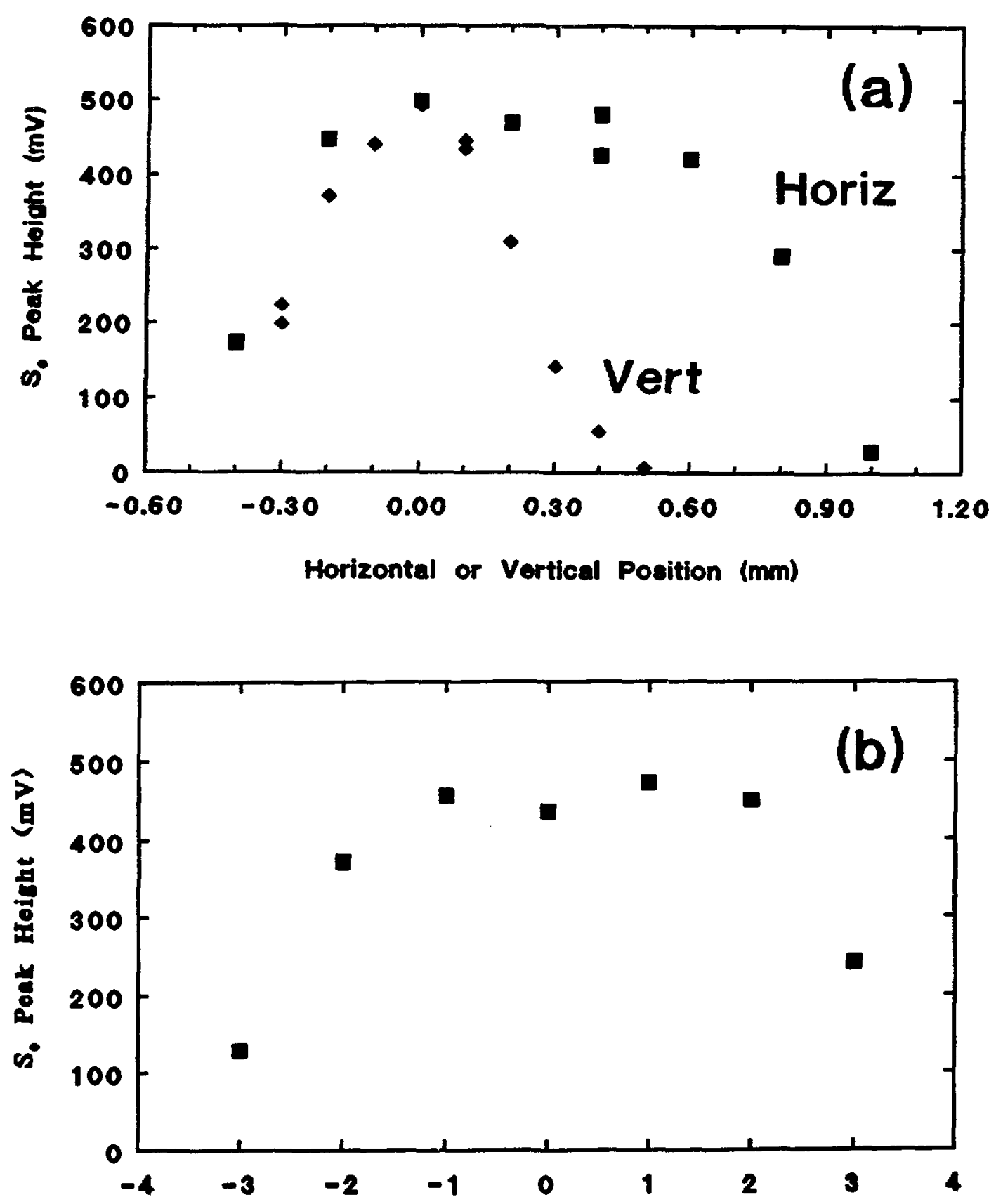

Axial Position (mm)

Figure 8. Height of the $S_{0}$ peak of atomic hydrogen as the focusing mirzor is translated through the point of maximum signal. (a) Motion perpendicular to the laser beam axis. The alignment tolerances are at least $\pm 100 \mu \mathrm{m}$ in any direction. (b) Motion along the laser beam axis. The alignment tolerance is $\pm 2 \mathrm{~mm}$ in this direction. 
the pinhole. The other measured the portion that passed through the pinhole. The photodiode signals could be analyzed in two ways to obtain two estimates of the spot size at a given distance from the mirror. The change in average transmission as the focal spot translated across the pinhole provided one measure of the size of the spot, when compared to a model that included alignment error and random motion of the spot. The maximum transmission among the individual measurements provided a second measure of spot size. The occasional peak value among hundreds of similar measurements must represent a direct hit on the pinhole, so the percentage transmitted indicated the spot radius of that single pulse, assuming only the functional form of intensity integrated over a disk. Since the pinhole was small compared to the measured spot radius, it made little difference whether a Gaussian waist or an Airy disk was assumed. In some sets of measurements, the two measures of spot size were the same within the estimated errors, and it is likely that the smallest spot size on any shot was near the average for that series of shots. In other sets, the average was larger than the single-shot estimate. The conclusion from these tests is that the spot was sometimes eight and sometimes ten times diffraction limited. Details of these measurements appear in Appendix C.

Based upon the measured temporal and spatial profiles of the UV beam, the irradiance distribution function in cylindrical coordinates could be described by the $M^{2}$ model of a multimode beam (Marshall 1971, Sasnett 1989):

$$
I(r, z, t)=I_{0} \frac{\operatorname{sech}^{2}\left(2 t / T_{p}\right)}{1+\left(M^{2} \lambda z / \pi W_{0}^{2}\right)^{2}} \exp \left\{-\frac{2 r^{2}}{W_{0}^{2}\left[1+\left(M^{2} \lambda z / \pi W_{0}^{2}\right)^{2}\right]}\right\} .
$$

In this model, $W_{0}$ is the waist radius defined by the $1 / e^{2}$ irradiance contour. $M^{2}$ describes the rate at which the beam expands, and it is equal to one for a $\mathrm{TEM}_{00}$ beam. The parameter $T_{p}$ is 1.14 times the FWHM pulse length, hence $T_{p}=700 \pm 50$ fsec. The peak irradiance $I_{0}$ is related to the pulse energy $E_{p}$ by

$$
I_{0}=\frac{2 E_{p}}{T_{p} \pi W_{0}^{2}} .
$$


When the beam was reliably achieving its best focus, I measured $W_{0}=35 \pm 2 \mu \mathrm{m}$ and $M^{2}=6.2 \pm 1$. At other times, I measured $W_{0}=45 \mu \mathrm{m}$, but could not establish a value for $M^{2}$.

\subsubsection{Density of atomic hydrogen}

The important thing to know about the atomic hydrogen beam was its density. This can be calculated as in Chan et al. (1988) from the pressure inside the discharge tube, the estimated conductances of the capillary, the process of effusion at the end of the capillary, and the angular size of the hole in the skimmer. This results in an estimate of $10^{10}$ atoms $/ \mathrm{cm}^{3}$ for the operating conditions in September of 1989 and somewhat lower values for later data collections. These calculations are in reasonable agreement with measurements of $\mathrm{H}_{2}$ pressures at the RGA, assuming only that the density decreases as the square of the distance from the end of the capillary. A further check is the number of electrons produced at the saturation point, where all of the atoms in the center of the focal volume have been ionized. All of these agree within factors of two.

Atomic hydrogen pressures were derived from RGA measurements by comparing molecular hydrogen pressures as the discharge was switched on and noting that each molecule that dissociates produces two atoms. The atomic density can also be derived from the mass 1 RGA reading, but the process is less direct. One reason the mass 2 method is more direct concerns a major advantage of quadrupole RGA's: the lower ionization efficiency for light molecules is compensated by the higher transmission factor of the quadrupole. This allows one to read mass 2 , mass 18 , and mass 28 pressures directly unless great accuracy is required. The case of atomic hydrogen is different, however. The ionization cross section of $\mathrm{H}_{2}$ is only $45 \%$ greater than that of $\mathrm{H}$ for $60 \mathrm{eV}$ electrons, but the overall sensitivity of the RGA was found to be seven times as great. This ratio is a measured quantity that adds to the error of the calculation. Further, a portion of the mass 1 reading is actually due to hydrogen molecules and water. 
Measuring and subtracting this portion again adds to the error. Details of these measurements appear in Appendix B.

The RGA did not produce absolute measurements of gas density, but the manufacturer attempted to make it read pressures in actual millibar units. This produced reliable results with the Faraday cup detector, but electron multipliers are subject to gain variations of several kinds, including chemical reactions with the gases in the vacuum system. For this reason, I used the Faraday cup for all measurements that did not require rapid sampling. The RGA agreed with the ion gauge in the lower chamber for nitrogen and molecular hydrogen at pressures in the $10^{-6}$ Torr range. When the RGA indicated pressures below a few times $10^{-8}$ Torr, the ion gauge reading was usually much higher, probably because the conductivity of the $38 \mathrm{~mm}$ tubing between the cube and the ion gauge became an important factor at these pressures. In these cases, the RGA reading was considered the better representation of conditions in the interaction region.

\subsubsection{Number of electrons produced per unit signal recorded}

In order to determine the number of electrons produced in the interaction region from the recorded signals, one must consider the fraction of electrons that enter the time-of-flight spectrometer (0.5), the transparencies of the spectrometer grids $(0.90,0.90$, and 0.82$)$, the detection efficiency of the detector, and the gain of the MCP assembly and its associated electronics, that is, the recorded signal per detected electron. The first two factors are known with good confidence from the design of the spectrometer, and typical MCP detection efficiencies for electrons accelerated to $250 \mathrm{~V}$ are $0.7 \pm 0.15$ (Fraser 1983). The product of these factors is

$$
F_{S}=\frac{1 \text { electron detected }}{4.3 \pm 0.9 \text { electrons produced }}
$$

The final factor is evaluated as described in Appendix $D$ as

$$
G_{M}=\frac{7.2 \pm 0.4 \mathrm{mV} \text { average signal }}{\text { detected electron }}
$$


when the power supply voltage is $2.209 \mathrm{kV}$, so the overall gain of the spectrometer system at this supply voltage is

$$
G_{S}=F_{S} G_{M}=\frac{1.7 \pm 0.3 \mathrm{mV} \text { average signal }}{1 \text { electron produced }}
$$

When the system gain has been measured, it is equally important to know that the gain is linear when many electrons arrive together and that the gain does not change because of fluctuations in the supply voltage. The latter condition was well satisfied. In a 14 -hour test, the power supply voltage was stable to $\pm 0.056 \%$, which means the MCP gain was stable to $\pm 0.55 \%$. The other condition, that the gain be linear, could not be checked because there was no independent way to count the incident electrons, but there were two reasons to believe it was satisfied. First, electrons from the interaction region, which never numbered more than 1000 , were spread over approximately 46000 channels of the MCP, making it very unlikely that two would strike the same channel. Second, the MCP signal was coupled out through a large $(1 \mu \mathrm{F})$ capacitor which could not be discharged appreciably by a pulse containing a few nanocoulombs at most.

The main concern in calibrating the MCP was therefore obtaining an accurate value of recorded signal per incident electron. The difficulty in doing this was that the signal itself was the only indication that an electron had arrived, and the histogram of signals showed that small responses were more likely than large ones. The average gain determined from these measurements therefore must include an estimate of how many electrons produced no signal. In order to refine this estimate, I divided the MCP signal between the input to the transient digitizer and an oscilloscope so that the oscilloscope could trigger on pulses that measured zerc on the digitizer. This introduced an uncertainty in how much of the signal was tapped off, but it did establish a mininum number of missed electrons that fixed one end of the confidence interval for the system gain. The 
other end was found by extrapolating the exponential trend in the signal histogram for small signals to the limit of zero signal. This procedure resulted in the calculated system gain of $7.2 \pm 0.4 \mathrm{mV}$ per incident electron at an applied voltage of $2.2 \mathrm{kV}$. Further details of these measurements, gains at higher applied voltages, and measurements of subsystem gains appear in Appendix D.

\subsubsection{Time-of-flight spectrometer accuracy and resolution}

The spectrometer has produced single $H$ peaks as narrow as $80 \mathrm{meV}$ full width at half maximum (FWHM) at $1.4 \mathrm{eV}(\Delta E / E=0.06)$. This width was obtained under conditions of $8 \times 10^{12} \mathrm{~W} / \mathrm{cm}^{2}$ irradiance and total gas pressures below $10^{-7}$ Torr, so that each laser pulse produced an average of 100 or fewer electrons at all energies. It thus represents the performance of the spectrometer in the absence of space charge. The spectrometer's resolution is determined by three components which add in quadrature. Using the form of Equation (29) of Kruit and Read (1983),

$$
\frac{1}{2} \frac{\Delta E}{E}=\frac{\Delta T}{T} \approx\left(a+b E+c E^{-2}\right)^{1 / 2}
$$

where $E$ is energy in $\mathrm{eV}, a=9 \times 10^{-4}, b=3.5 \times 10^{-5} \mathrm{~cm}^{-1}$, and $c=2.5 \times$ $10^{-5} \mathrm{~cm}^{-2}$.

The $c$ coefficient represents voltage transients on the grids as the laser fired and differences in contact potential across the drift tube. Transients were held to $\pm 10 \mathrm{mV}$ by shielding the power supply. Differences in contact potential were minimized by a carbon coating and were believed to be smaller than the voltage transients. The $b$ coefficient represents the finite sampling rate of $100 \mathrm{MHz}$, which contributed $17 \mathrm{meV}$ to the width at $1.4 \mathrm{eV}$. This leaves $97 \%$ of the observed width attributed to coefficient $a$, which represents variations in flight time among electrons of equal energy. The size of coefficient $a$ depends on the distribution of initial directions of flight, the shape of the magnetic field near the magnet, and the exact placement of the laser focus within the field. In this apparatus, 
the electric field of the laser was perpendicular to the spectrometer axis, so ATI electrons were emitted preferentially in directions that maximized the variation (Kruit and Read 1983). The $a$ coefficient was expected to be dominant, but it was not expected to be so large. Calculations of electron trajectories, assuming perfect alignment and no other source of broadening, predicted $a=2 \times 10^{-4}$, hence $\Delta E / E=0.03$.

\subsection{Operation}

Collecting electron spectra was fairly straightforward process, provided steps were taken before and during the operating period to avoid certain problems. These steps are described in the following paragraphs.

One of the potential problems involved the system which cooled the walls of the discharge tube with water at $20^{\circ} \mathrm{C}$. Bubbles in the water, which changed the resonant frequency of the cavity as they passed through it, were eliminated by circulating the water for several hours before the discharge was started. Normally, the water flow was stopped only for maintenance. When the chamber walls were being baked, the water temperature was raised to $50^{\circ} \mathrm{C}$ to prevent contaminants from the walls from migrating to the discharge tube.

We also tried in many other ways to keep the upper chamber clean. After hydrogen atoms are produced in the discharge, they may recombine on the walls of the discharge tube and capillary, as well as on other surfaces after they emerge, but the rate of recombination on Pyrex is very low. The manufacturer claims a typical dissociation fraction of $90 \%$ at the outlet of the capillary when the glass is thoroughly clean. Our performance, measured beyond the skimmer at the RGA, was closer to $25 \%$ until we cleaned the glass with hydrofluoric acid as suggested in the operating instructions. Then it was about 50\%. I coated the stainless steel skimmer with Teflon to prevent recombination there, but there was no measurable change. It now appears that most of the recombination took place in the lower chamber. 
When the discharge operated, the walls became warm even with water cooling, causing partial pressures of water and nitrogen in the lower chamber to increase for many minutes after the start of continuous operation. For this reason, I operated the discharge for a while before taking data. The wall temperature stabilized much more quickly when the discharge was pulsed.

When operating an RGA in a cryopumped system, it is necessary to accommodate the slow cycle of the pump, about $2 \mathrm{~Hz}$. The table vibrated strongly at this frequency, producing currents in the RGA cable. After amplification, these appeared as random pressure readings at all masses. One must set the RGA to average hundreds of readings to remove this noise, which reduces its ability to follow rapid changes in pressures. Further, the actual pressure of $\mathrm{H}_{2}$ followed the cryopump cycle, since its vapor pressure is greater than one torr even at $10^{\circ} \mathrm{K}$. (Cryopumps do not freeze out $\mathrm{H}_{2}, \mathrm{He}$, and $\mathrm{Ne}$ as they do other gases, but adsorb them on activated charcoal.) This made it hard to measure the amount of $\mathrm{H}_{2}$ that dissociated when the discharge came on. One way to avoid the problem was to ise the electron multiplier, so that vibration noise could be averaged out quickly compared to the pump cycle, and observe departures from the sine wave pattern when the discharge started. Another way was to pulse the discharge at nearly the cryopump period and observe the beats in the pressure. This worked because the change due to dissociation was about equal to the change due to the pump cycle. These high-speed measurements were then used to interpret the slow-speed readings taken during data collection. The strong vibrations in the table also affected the joulemeter, which was microphonic, but placing it on a different table made it reliable.

It was necessary to monitor the average energy per laser pulse during each data collection. The maximum length of a data run varied from a few minutes to more than an hour depending on the condition of the laser system. Data collection had to stop from time to time while the system's timing was adjusted. 
Also, by the end of three hours' operation, about half of the original charge of fluorine in the $\mathrm{KrF}$ amplifiers had been converted to stable fluorides or otherwise rendered unavailable, causing the average energy to decrease. Normally, fluorine was added at noon, and the gas was replaced each morning.

It was also necessary to control the gas density at the interaction region, holding it constant during each data run at a value that produced a reasonably large signal without overloading the detector or causing too much space charge. The gas density could be controlled in two ways. First, the heating current in the $\mathrm{Pd}$ filter and the $\mathrm{H}_{2}$ supply pressure determined the pressure in the discharge tube. This pressure could be varied over approximately a factor of eight. After a few minutes of equilibration, it could be held steady to $\pm 3 \%$ by occasional adjustment of the sapphire valve. Second, raising the effusive source placed a lower density portion of the flow at the interaction region. The range of adjustment, from $12 \mathrm{~mm}$ to $36 \mathrm{~mm}$ above the interaction region, provided a factor of one to nine in density. The two adjustments thus allowed the hydrogen density in the interaction region to be varied over two orders of magnitude, from $10^{9}$ to $10^{11}$ atoms $/ \mathrm{cm}^{3}$. Only the lower decade of densities were useful in ionization rate measurements, however, because of the need to keep the total number of ionizations low.

The procedure for collecting electron spectra was as follows: I started the gas flow and the discharge several minutes to an hour before taking data to give the pressures and temperatures time to stabilize while I checked that the other equipment was working properly. When the UV beain was ready, I verified its alignment with the red beam, then reduced its diameter to $30.5 \mathrm{~mm}$ (1.20 in.) with an aperture to ensure a circular focal spot. Then I translated the focusing mirror to place the focal region at the point that produced the highest $S_{0}$ peak in atomic hydrogen. When the RGA indicated that all gas pressures were stable, I attenuated the UV beam as needed and started one of the data collection 
programs to operate the equipment and record the data. During each collection, I checked the gas pressures and the average pulse energy frequently.

\subsection{Additional considerations}

\subsubsection{Background gases}

As one would expect, the major background gases were nitrogen and water, with the balance composed mainly of hydrogen and oxygen. The relative concentrations of minor gases varies with the length of time since exposure to the atmosphere or use of hydrogen in the experiment. Other "universal contaminants" such as argon and carbon dioxide were present only in subnanotorr amounts. Hydrocarbon contamination was held to very low levels by using a cryopump in the lower chamber and a turbopump in the upper chamber, with liquid nitrogen cold traps in the turbopump's foreline and the cryopump's regeneration system. Background gas readings were routinely recorded before and after an experimental run.

During the discharge, the RGA recorded considerable numbers of molecules of masses 29, 31 and 43. Indicated partial pressures often exceeded those of the familiar masses $28\left(\mathrm{~N}_{2}\right), 32\left(\mathrm{O}_{2}\right)$, and $44\left(\mathrm{CO}_{2}\right)$. Rates of removal after the discharge ended were typical of pumping rates, suggesting that the new molecules were stable. Candidate species, considering the atoms available, are $\mathrm{HN}_{2}, \mathrm{HON}$, HOCN, and $\mathrm{HN}_{3}$. (Similar amounts of $\mathrm{H}_{2} \mathrm{O}$ and $\mathrm{NH}_{3}$ that might have been formed in the discharge would have been masked by signals from background water at masses 17 and 18.) While these molecules were easily detectable, they were far outrumbered by $\mathrm{H}, \mathrm{H}_{2}$, and $\mathrm{H}_{2} \mathrm{O}$, and there is no evidence that they influenced the ATI spectra.

\subsubsection{Limitations on temperatures for baking}

Baking temperatures were limited by the practice of leaving water in the jacket of the discharge tube and by certain materials used in the lower chamber 
and the drift tube. Rather than draining the cooling water when the walls of the upper chamber were being baked, we left it circulating, though at the increased temperature of $50^{\circ} \mathrm{C}$. The walls then had to remain cooler than this to prevent contaminants from migrating to the discharge tube. The hydrogen source could otherwise have been baked at $200^{\circ} \mathrm{C}$. Temperatures in the lower chamber were limited to $90^{\circ} \mathrm{C}$ for two reasons. The insulation of the magnet wire that formed the solenoid around the drift tube melted at $105^{\circ} \mathrm{C}$. Also, the screw holding the in-vacuum mirror became loose at $90^{\circ} \mathrm{C}$ and allowed the mirror to turn. Since the apparatus could not be baked at high temperatures, the best background pressures achieved in the interaction region were in the low $10^{-9}$ Torr range.

\subsubsection{Transients in RGA readings}

The dissociation fraction measured at the RGA showed a transient effect when the discharge started. Figure 9 shows partial pressures measured at 24 samples per second as the discharge was flashed on for $150 \mathrm{~ms}$ every second. The $\mathrm{H}_{2}$ trace shows the cryopump cycle very clearly, but a long-period average was needed to see the decrease due to dissociation. The $\mathrm{H}$ trace increases rapidly to a maximum at the start of the discharge, then decreases to a steady level.

This behavior suggests that many of the $\mathrm{H}$ atoms streaming toward the cryopump reflected off the first stage of the pump, collected on the walls of the lower chamber, and recombined on the walls when they reached the necessary areal density. The first stage of the pump was designed to force approaching molecules to reflect at least once before reaching the colder second stage. In addition, the center of the first stage, which was centered on the $\mathrm{H}$ beam, was a solid plate. It was unlikely, therefore, that an atom could reach the second stage on its first attempt. As atoms began to enter the lower chamber, their pressure would increase toward the point of equilibriun with the speed of the cryopump for $\mathrm{H}$ atoms. This increase would initially be unaffected by recombination, because there would be no atoms on the walls. As the walls became decorated with atoms, 


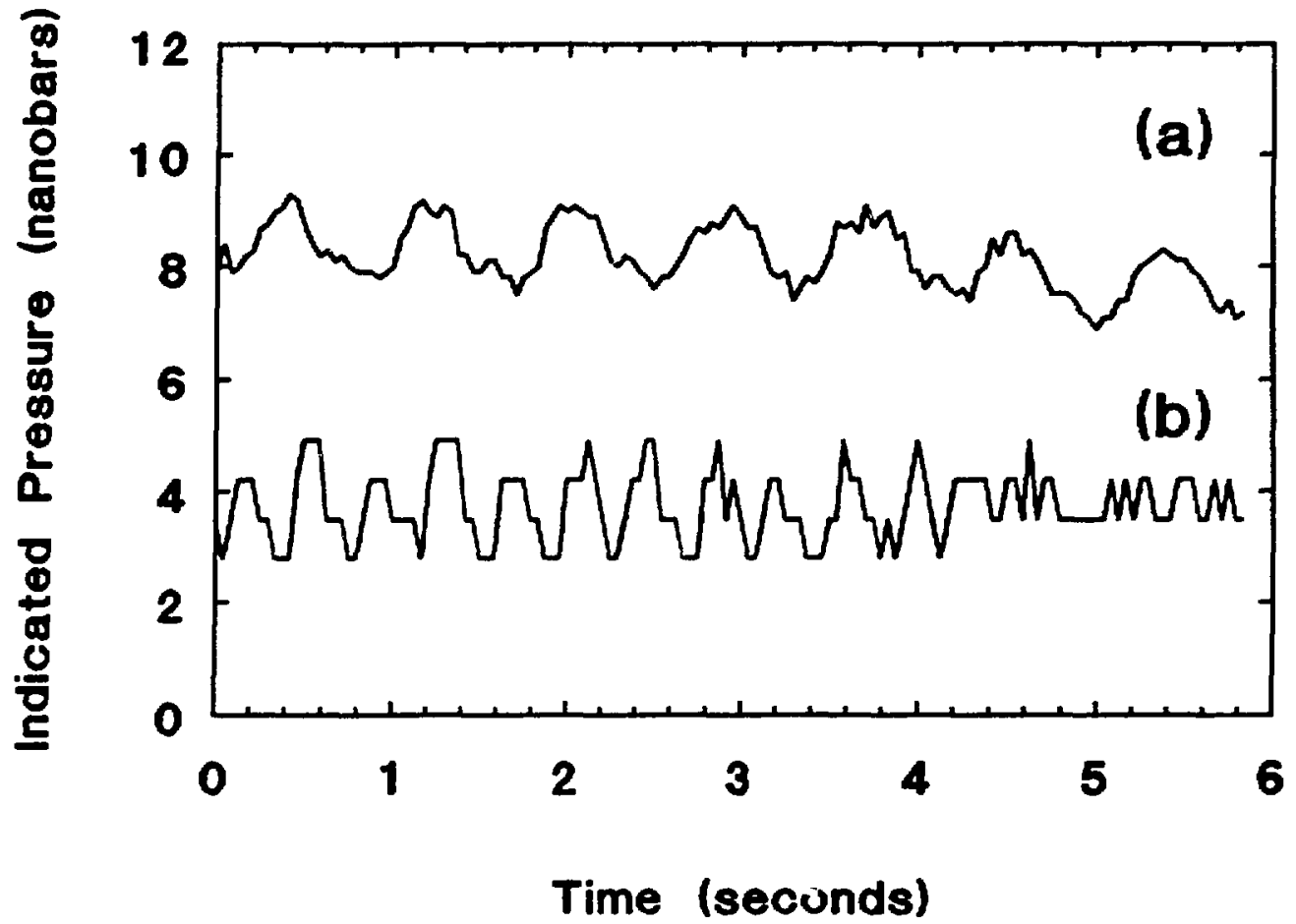

Figure 9. Partial pressures of (a) Mass-2 $\left(\mathrm{H}_{2}\right)$ and (b) Mass-1 (H) as the discharge was flashed on for $150 \mathrm{msec}$ every $400 \mathrm{msec}$. The discharge remained off after $4.2 \mathrm{sec}$. Data were collected at 24 samples per second on 9 May 90 . The $\mathrm{H}_{2}$ trace shows the cryopump cycle very clearly. The $\mathrm{H}$ trace increases rapidly to a maximum at the start of the discharge, then decreases to a steady level. The $\mathrm{H}$ trace is multiplied by 7 to compensate for the lower sensitivity to $H$, but it is not corrected for the portion of the mass- 1 signal due to $\mathrm{H}_{2}$.

the point of equilibrium would shift. The pumping speed for $\mathrm{H}$ in the presence of recombination is often taken to be twice the speed for $\mathrm{H}_{2}$, since each recombined molecule that is pumped removes two atoms. Eventually, the number of atoms in the chamber and the number of atoms on the wall would stabilize at values for which the overall pumping rate for separate and recombined atoms equaled the 
rate atoms entered the chamber. The steady state reading of the RGA was then the better measure of $\mathrm{H}$ density at the interaction point, because the density of scattered atoms, being uniform through the chamber, was a smaller portion of the total density near the skimmer than at the RGA. This conclusion is supported by the fact that ATI spectra taken during a steady discharge are no different from those taken at the end of a pulsed discharge when the $\mathrm{RGA}$ reading for $\mathrm{H}$ was $30 \%$ higher.

An estimate of the rate of recombination on the walls indicates that $100 \mathrm{~ms}$ is a reasonable time in which to reach equilibrium. This estimate is based on the observation that nitrogen at $10^{-6}$ Torr forms a monolayer in one second. Atomic hydrogen at $4 \times 10^{-9}$ Torr, moving five times as fast, might cover as much as $0.2 \%$ of the wall in $100 \mathrm{~ms}$. Let us call the fraction covered $F_{c}$ and assume that $F_{c}=0.002$ at equilibrium. The rate of collisions with the wall, for a characteristic chamber dimension of $26 \mathrm{~cm}$ and mean speed of $2.6 \times 10^{5} \mathrm{~cm} / \mathrm{sec}$, is $R_{c}=10^{4}$ per second per atom. The total rate of collisions when $N_{e}$ atoms are present is $N_{e} R_{c}$. If the probability of recombination $P_{r}$ when two atoms meet at the wall is $70 \%$, the recombination rate at equilibrium is

$$
R_{e}=N_{e} R_{c} P_{r} F_{c}=-14 N_{e} \sec ^{-1}
$$

Since this is the equilibrium condition, $R_{e}$ is also the net rate at which atoms enter the chamber (the total rate of entry minus the rate at which uncombined atoms are pumped out). If the number of atoms $N(t)$ at some time $t=t_{0}$ should be slightly greater than the equilibrium value $N_{e}$, but not so much greater that $F_{c}$ is increased, the excess $\delta N=N\left(t_{0}\right)-N_{e}$ is removed by the difference between the net entry rate $R_{e}$ and the instantaneous recombination rate $R(t)$ :

$$
\frac{d(\delta N)}{d t}=R_{e}-R\left(t_{0}\right)=\left(N_{e}-N\left(t_{0}\right)\right) R_{c} P_{r} F_{c}=(\delta N) R_{c} P_{r} F_{c} .
$$


$N$ thus approaches equilibrium with a characteristic time of $\tau$ :

$$
N(t)-N_{e}=(\delta N) e^{-\left(t-t_{0}\right) / \tau}, \text { where } \tau=70 \text { msec. }
$$

This model of recombination, with $F_{c}$ increased to 0.01 , agrees with estimates of the flow rates into and out of the lower chamber. This comparison appears in Appendix B.

\subsubsection{Pulse stretching by the windows}

Windows lengthen short pulses by delaying their higher frequency components more or less than their lower frequency components. In this case, however, the pulse was stretched by less than $4 \%$ of its initial length. The flight time $t$ of a pulse of wavelength $\lambda$ across a distance $L$ is determined by the group velocity $v_{g}$ :

$$
t=\frac{L}{v_{g}}=L \frac{d k}{d \omega}
$$

where $\omega$ is the angular frequency, $k=2 \pi / \lambda=\omega n / c$ is the wave number, $n$ is the index of refraction, and $c$ is the speed of light in vacuum. Then

$$
t=L \frac{d}{d \omega}\left(\frac{\omega n}{c}\right)=\frac{L}{c}\left(n+\omega \frac{d n}{d \omega}\right)=\frac{L}{c}\left(n-\lambda \frac{d n}{d \lambda}\right) .
$$

The difference in the flight times of two components of the pulse with wavelengths $\lambda_{1}$ and $\lambda_{2}=\lambda_{1}+\Delta \lambda$ is thus

$$
\Delta t=\frac{L}{c}\left(\lambda \frac{d n}{d \lambda}\right)_{\lambda=\lambda_{1}}-\frac{L}{c}\left(\lambda \frac{d n}{d \lambda}\right)_{\lambda=\lambda_{2}} .
$$

If $d n / d \lambda$ varies slowly, as it does for fused silica near $\lambda=248 \mathrm{~nm}$ (Weast 1965),

$$
\Delta t \approx \frac{L}{c} \frac{d n}{d \lambda} \Delta \lambda
$$

In this experiment, $d n / d \lambda=0.52 \mu \mathrm{m}^{-1}, \Delta \lambda=0.3 \mathrm{~nm}$ FWHM (Roberts et al. 1988), and the total path length $L=38 \mathrm{~mm}$, so

$$
\Delta t=20 \text { fsec FWHM, }
$$

which is much less than the pulse length of $600 \mathrm{fs}$ FWHM. 


\section{Data, analysis, and conclusions}

\subsection{Features of the electron spectra}

Figure 10 shows a pair of electron time-of-fight spectra collected with the discharge producing atomic hydrogen (solid curves) and with the discharge off (dashed curves). The transient digitizer output is shown as it was recorded, with signal in millivolts and time in microseconds. Time zero is defined by the detection of scattered UV light from the laser pulse. Electrons of different energy reach the detector at different times, the most energetic arriving first. At late times come photoelectrons from the retarding grids. These data were collected with a retarding potential of $4 \mathrm{~V}$, so the non-ATI $\left(\mathbf{S}_{0}\right)$ electrons from $\mathrm{H}$ were rejected. The $S_{1}$ and $S_{2}$ peaks are very clear in the discharge-on trace. Two sets of ATI electrons from $\mathrm{H}_{2}$ also appear in the figure. These peaks, labeled $M_{1}$ and $M_{2}$, became smaller when the discharge came on. A few $M_{0}$ electrons were probably collected, but they were mixed together with the photoelectrons from the grids. Likewise, electrons from the nitrogen background overlapped the molecular hydrogen peaks because the ionization energies of the two molecules are about $0.1 \mathrm{eV}$ apart. The nitrogen signals were much smaller, however. Molecular peaks are much broader than atomic peaks because of the larger number of transitions available. The structure within each molecular hydrogen peak is discussed in a later section, as are the methods used to identify the sources of the various peaks.

Figure 11 shows the same spectra plotted as signal per unit energy vs. electron energy. Here the fastest electrons appear toward the right, and the shapes of the peaks are different, but the area of each peak is still proportional to the number of electrons that arrived together. In this representation, the widths 


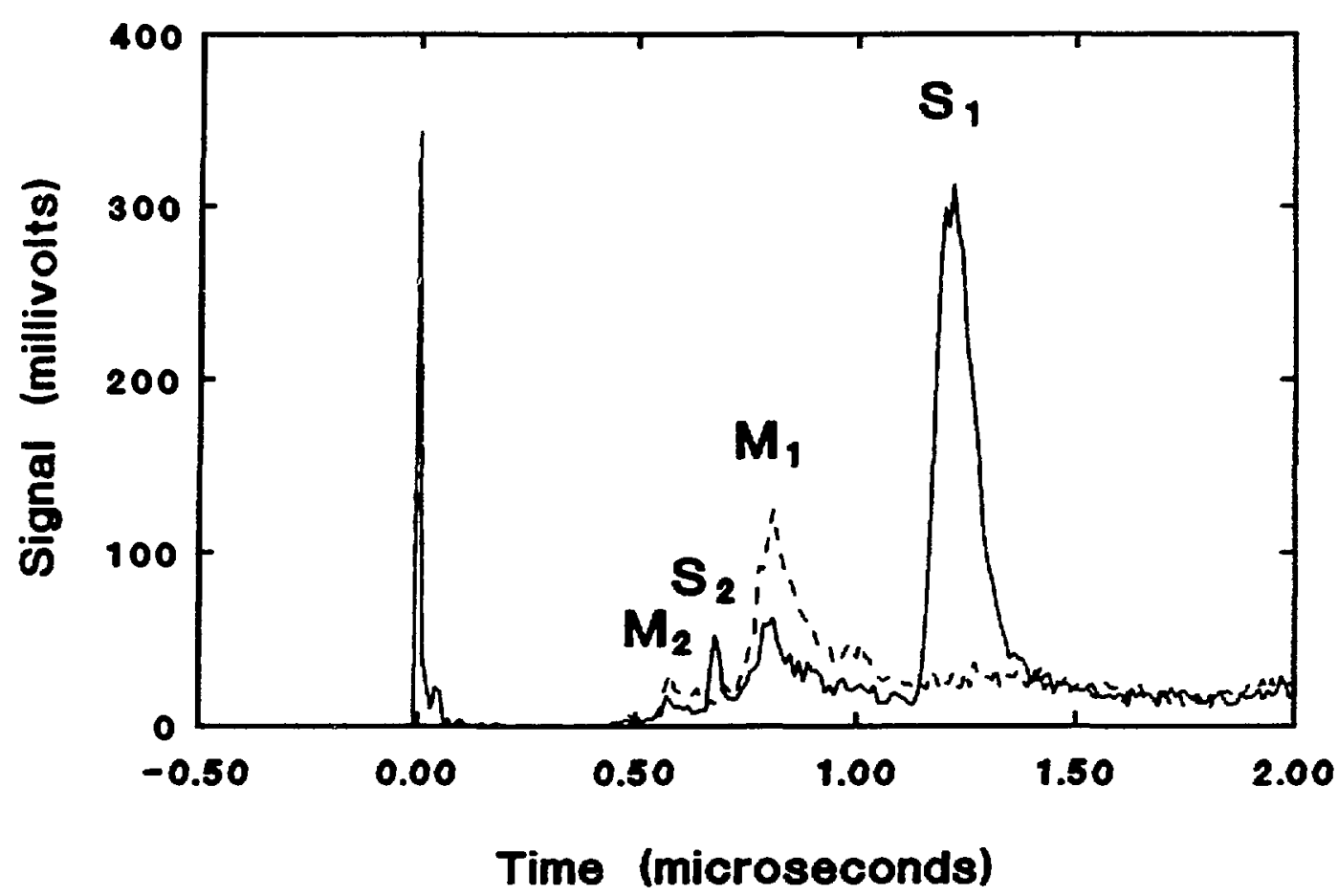

Figure 10. Electron spectra, recorded with a $4 \mathrm{~V}$ retarding potential, vs. time of flight. The solid curve, taken with the discharge on, contains large ATI signals from atomic hydrogen. The dashed curve, taken with the discharge off, displays ATI signals from molecular hydrogen and impurities only. The pulse from detection of scattered light at time zero marked the beginning of the drift period. The first and second ATI peaks of atomic and molecular hydrogen $\left(S_{1}, S_{2}, M_{1}\right.$, and $M_{2}$, respectively) are marked. The non-ATI peaks ( $S_{0}$ and $\left.M_{0}\right)$ were stopped by the retarding potential.

and separations of the peaks can easily be read in energy units. It is easier to find successive peaks in each ATI series (or "ladder"), such as those at 2.5 and $7.5 \mathrm{eV}$ due to water $\left(W_{1}\right.$ and $\left.W_{2}\right)$. Also, the distinction between the atomic peaks and the background at low energies is more visible. The logarithmic scale in Fig. 11 emphasizes the similarities within each ATI ladder. The same general curve shapes are repeated, and the decrease in signal level from one peak to the 


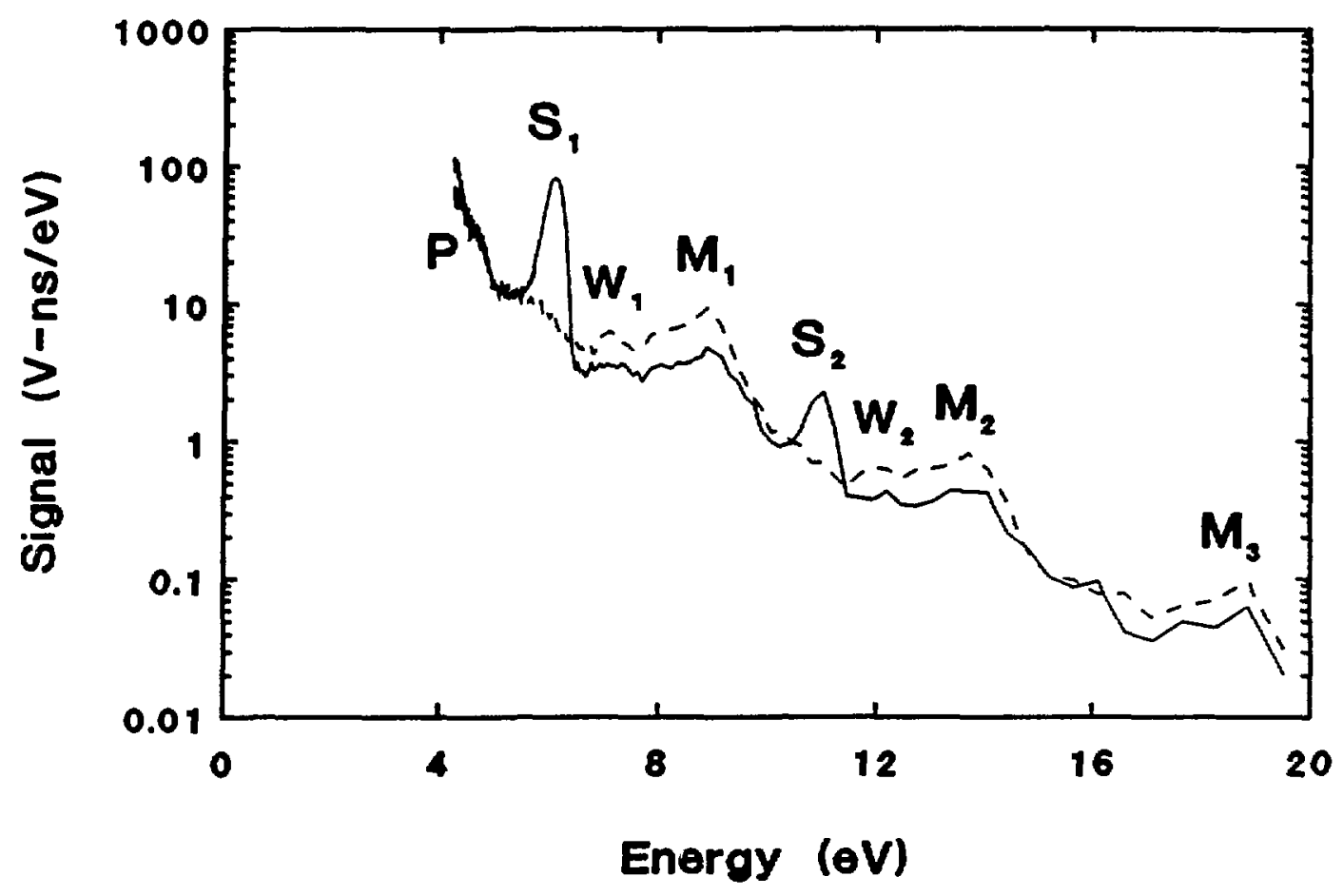

Figure 11. Electron spectra, recorded with a $4 \mathrm{~V}$ retarding potential, vs. electron energy. These are the same spectra as in Fig. 10, but converted to signal per unit energy and plotted on a logarithmic scale. Two peaks due to water $\left(W_{1}\right.$ and $\left.W_{2}\right)$, a third peak due to $H_{2}$, and the signal from photoelectrons $(P)$ are marked.

next is about the same through each ladder, though not necessarily the same in different ladders.

\subsection{Effect of different values of peak irradiance}

Figure 12 compares an electron spectrum collected at high peak irradiance to one collected at low peak irradiance. All signals increase with irradiance, though at different rates, depending on the nature and order of the process involved. Scattered light and photoelectron contributions are linear in pulse energy. $M_{1}$ ATI signals increase more quickly than $M_{0}$ signals, and $M_{2}$ more quickly than $M_{1}$, so the overall slope of the curve is less at higher irradiance. An important point 
is that ATI signals depend upon irradiance, not energy. The size of the step in signal level going from $M_{0}$ to $M_{1}$ to $M_{2}$ provides a rough estimate of irradiance that can be compared to the value calculated from the pulse energy to test whether the parameters in the energy-to-irradiance conversion have remained constant.

In some cases, the irradiance did not remain proportional to the pulse energy. The four electron spectra displayed in Fig. 13 and summarized in Table 2 were intended to be identical, but each was stored separately to guard against just such a possibility. The energy of each laser pulse was measured, and all the other parameters that could be measured, such as the partial pressures of the major gases, remained within their normai bounds, yet it is apparent that the number of electrons produced changed considerably, especially in the few minutes between the third and fourth collections. Not only did the peaks become smaller, but also the ratio of the $S_{0}$ and $S_{1}$ peak areas decreased, and the crest of the $S_{0}$ peak shifted to a lower energy. This indicates that the change was not in the gain of the MCP or any other linear factor. As further proof of this, the scattered light peak and the photoelectron peak, which should be proportional to energy rather than irradiance, did not decrease. If the decrease in electron production was caused entirely by a change in the wajst radius $W_{0}$, the radius must have increased by a factor of 1.8 , which would take a $35 \mu \mathrm{m}$ waist to $63 \mu \mathrm{m}$ and decrease the irradiance by a factor of 3.2. On the other hand, a decrease in the irradiance should increase the $S_{0} / S_{1}$ ratio and shift the maximum of the $S_{0}$ peak to a higher energy. The observed peak shift, interpreted as a change in ponderomotive potential, suggests an increase of $9 \times 10^{12} \mathrm{~W} / \mathrm{cm}^{2}$, or $17 \%$ of the irradiance calculated from the pulse energy. One could speculate on what caused the change in these spectra, but the question probably cannot be put to rest without measurements of the laser beam that were not available. The parameters that were measured on $4 \mathrm{~J}$ un90 were mirror position, solenoid current, Helmholtz 
Chapter 4. Data, analysis, and conclusions

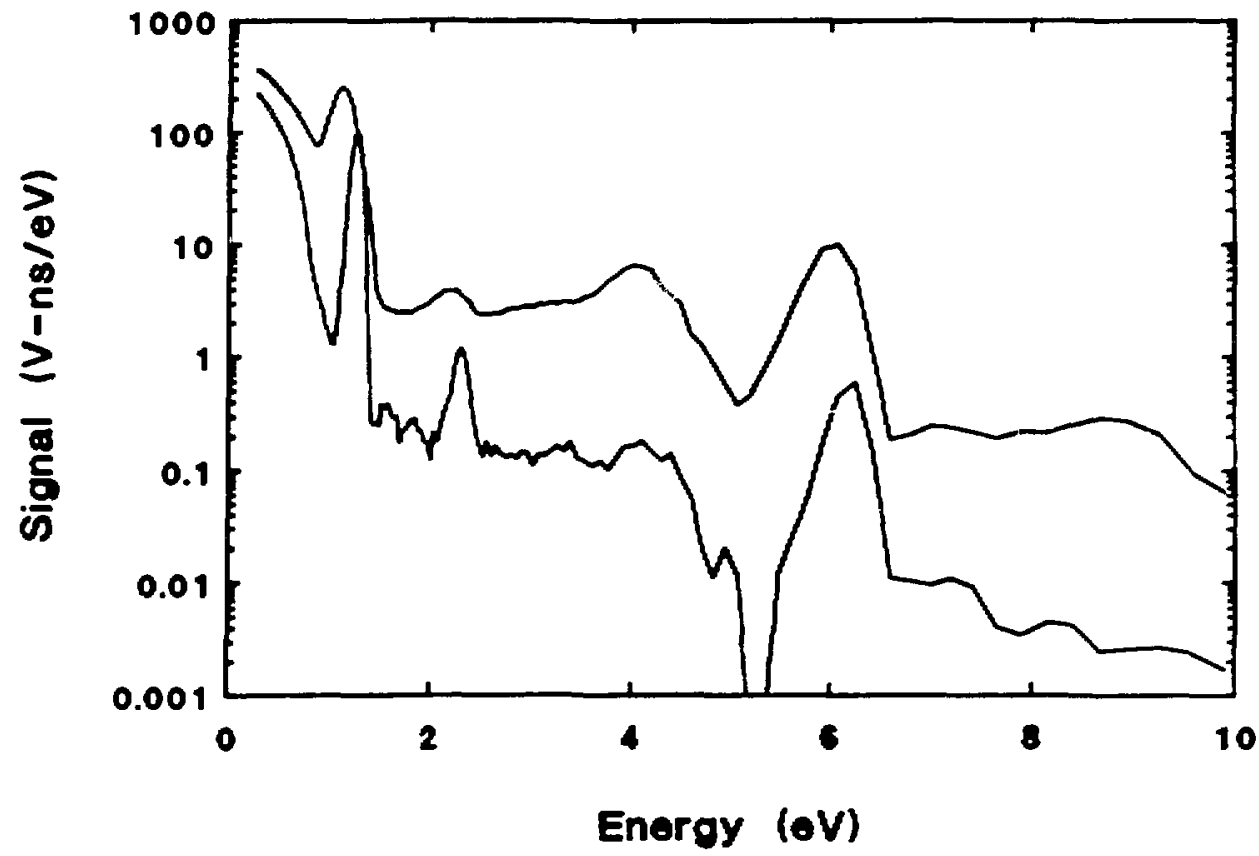

Figure 12. Electron spectra collected at different irradiances. The higher curve was collected at a peak irradiance of $2 \times 10^{14} \mathrm{~W} / \mathrm{cm}^{2}$, the lower at $4 \times 10^{13} \mathrm{~W} / \mathrm{cm}^{2}$.

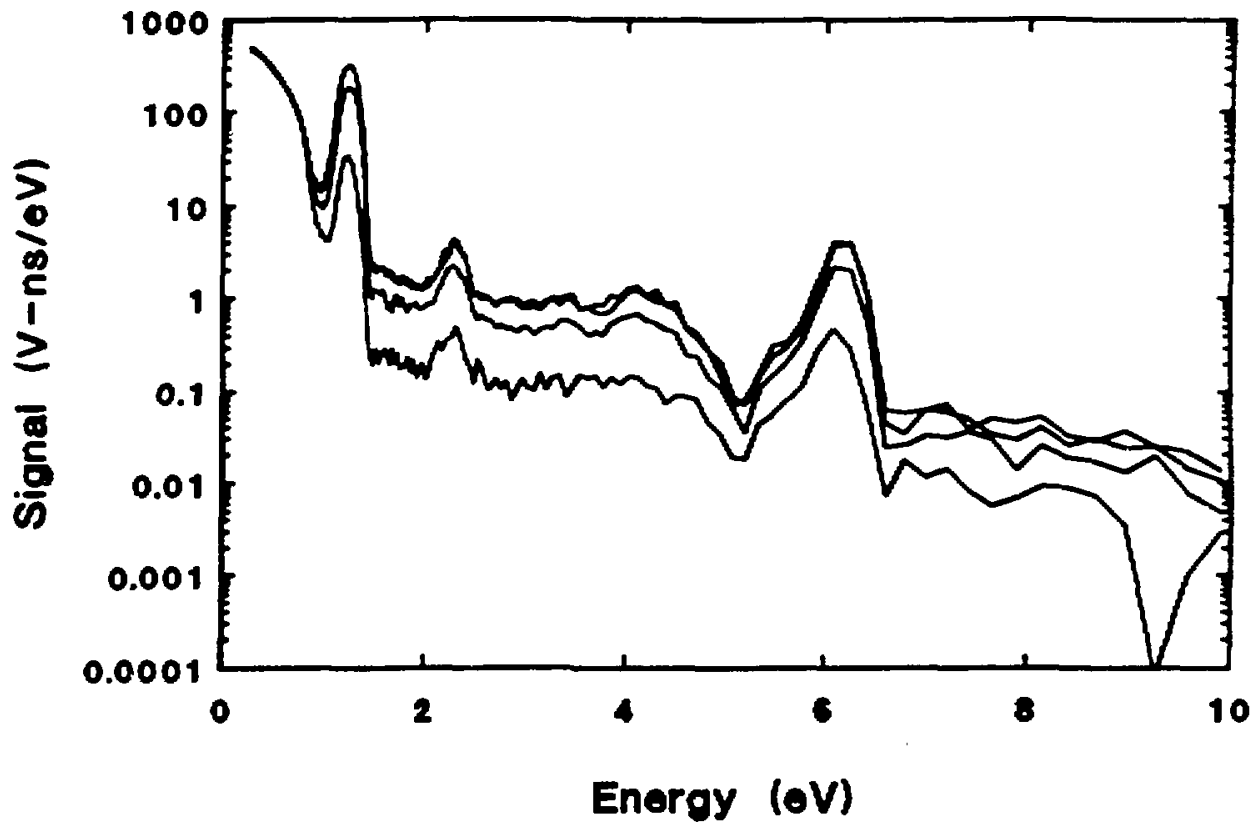

Figure 13. Four electron spectra showing a change in number of electrons produced with no change in pulse energy. The two highest curves were collected first, and the lowest curve was collected last. 
coil current, $\mathrm{H}_{2}$ supply pressure, cryopump temperature, duty cycle of the $\mathrm{rf}$ discharge, MCP voltage, and RGA readings at masses $1,2,18,28$, and 32 . In addition, no liquid nitrogen was being used near the table, so the temperature of the table did not change suddenly.

Table 2. Series of electron spectra collected $4 \mathrm{Jun} 90$ showing a change in number of electrons produced with no change in pulse energy. All measurable parameters were within normal tolerances. Position of the $S_{0}$ crest is $\pm 0.008 \mathrm{eV}$. Average irradiance was calculated from the distribution of readings among the energy bins.

\begin{tabular}{ccccccc}
\hline \hline $\begin{array}{c}\text { Start time } \\
\text { (hh:mm) }\end{array}$ & $\begin{array}{c}\text { Stop time } \\
(\text { hh:mm })\end{array}$ & $\begin{array}{c}\text { Crest of } \mathrm{S}_{0} \\
(\mathrm{eV})\end{array}$ & $\begin{array}{c}\text { Irradiance } \\
\left(\mathrm{W} / \mathrm{cm}^{2}\right)\end{array}$ & \multicolumn{3}{c}{ Electrons per shot } \\
$\mathrm{S}_{0}$ & $\mathrm{~S}_{1}$ & Ratio \\
\hline $15: 38$ & $15: 46$ & 1.262 & $5.2 \times 10^{13}$ & 893.5 & 23.0 & 38.8 \\
$15: 48$ & $15: 56$ & 1.262 & $5.3 \times 10^{13}$ & 926.4 & 24.8 & 37.4 \\
$16: 06$ & $16: 14$ & 1.262 & $5.1 \times 10^{13}$ & 506 & 13.2 & 38.4 \\
$16: 15$ & $16: 23$ & 1.239 & $5.0 \times 10^{13}$ & 86 & 2.6 & 33.1 \\
\hline \hline
\end{tabular}

When changes like this have occurred, comparison spectra taken in the next data session (in this case, the first two collections on 12Jun90) have matched the higher electron counts. This suggests that the LABS-I laser departed from its measured behavior as a consequence of some temporary condition. In these cases, the higher data are assumed to represent normal conditions, and the lower data are not used. 


\subsection{Isolation of atomic hydrogen signal from other signals}

There were three identifiable sources of electrons besides the source of interest, atomic hydrogen. There was an unavoidable background of hydrogen molecules and other molecules, principally water and nitrogen. Photoelectrons from the retarding grids arrived in great numbers together with low energy electrons from the interaction region. Finally, there was a small dark current due to electrons from the RGA, cosmic rays, radioactivity in the MCP glass, and electronic noise.

Signals from atoms and molecules could be identified by varying the concentrations of the various species. Atomic and molecular hydrogen were easily varied over wide ranges by changing the temperature of the palladium filter, moving the discharge tube away from the skimmer, and gating the discharge on and off. Densities of other gases, measured by the RGA, were high when the apparatus had recently been filled with air or nitrogen, and they declined with baking and pumping.

Photoelectrons from the grids could be identified in two ways. One was turning off the current in the Helmholtz coils, leaving the solenoid current on. This threw electrons from the interaction region off the MCP entirely. Photoelectrons from the center of the grid, however, were simply replaced by photoelectrons from some other part of the grid, since the entire grid was illuminated by scattered light. If the solenoid current was also off, photoelectrons from the walls of the drift tube followed the nearly vertical terrestrial magnetic field and reached the MCP very quickly. The other way to identify photoelectrons was to vary the retarding voltage. Photoelectrons from the charged grid were unaffected, while those from the grounded grid were either repelled or accelerated, acceleration providing the more dramatic results. If the electrons are assumed to originate at the grids, they are seen to have initial energies of 0 to $0.2 \mathrm{eV}$ that are shifted by the grid potential. This is in agreement with published work functions of 
carbon and gold, which are both near $14.8 \mathrm{~V}$ (Weast 1965). On the other hand, if the electrons are assumed to originate at the interaction region, their arrival times for different grid voltages are inconsistent. The photoelectrons were also conspicuous by their lack of variation with gas pressure in the interaction region.

Dark current could be observed in the MCP output with an oscilloscope. When the solenoid current was off, the count rate was less than one per second whether or not the ionization gauges and the RGA were operating. With solenoid current, RGA electrons could be brought to the MCP at rates too high for the oscilloscope to trigger on individually, but by displaying each trace at $1 \mu \mathrm{s} / \mathrm{cm}, \mathrm{I}$ found that the arrival rate was much less than one per microsecond. Since these electrons were not synchronized with the laser, they may be ignored in data analysis. Electrons from the ionization gauges were even less important because of their longer, less direct paths to the MCP.

Considering these sources of electrons, the total rate $R$ of electron detections at each derived electron energy $E$ can be written as

$$
R\left(E, I, V_{g}, \rho_{i}\right)=D+P\left(E, V_{g}\right)+\sum_{i} \epsilon_{i}(E, I) \rho_{i}
$$

where $I=$ laser irradiance,

$V_{g}=$ grid voltage,

$\rho_{i}=$ density at the interaction region of species $i$,

$i=1$ for $\mathrm{H}, 2$ for $\mathrm{H}_{2}, 18$ for $\mathrm{H}_{2} \mathrm{O}$, etc.,

$D=$ dark signal,

$P=$ photoelectron signal, which appears to have a distribution over $E$ that varies with $V_{g}$, and

$\epsilon_{i}=$ efficiency of producing electrons of energy $E$ from molecule $i$

at irradiance $I$, which is the quantity of interest.

It was usually possible to obtain a series of spectra with all parameters constant except $\rho_{1}$ (atomic hydrogen), $\rho_{2}$ (molecular hydrogen), and $\rho_{18}$ (water). 
The spectrum with the discharge off, denoted $R^{0}$, represents densities $\rho_{1}=0$, $\rho_{2}=R_{2}^{0}, \rho_{18}=R_{18}^{0}$, etc. Then

$$
\begin{gathered}
R\left(E, I, \rho_{i}\right)=R^{0}+\epsilon_{1}(E, I) \rho_{1}+\epsilon_{2}(E, I)\left(\rho_{2}-\rho_{2}^{0}\right)+\epsilon_{18}(E, I)\left(\rho_{18}-\rho_{18}^{0}\right) \\
\epsilon_{1}(E, I)=\left(1 / \rho_{1}\right)\left[R\left(E, I, \rho_{i}\right)-R^{0}-\epsilon_{2}(E, I)\left(\rho_{2}-\rho_{2}^{0}\right)-\epsilon_{18}(E, I)\left(\rho_{18}-\rho_{18}^{0}\right)\right]
\end{gathered}
$$

Since the spectra of $\mathrm{H}$ and $\mathrm{H}_{2} \mathrm{O}$ were found to be well separated in electron energy, it was possible to consider the water component to be part of $R^{0}$ and remove the resulting error in $\epsilon_{1}$ at the end of the calculation. Evaluation of $\epsilon_{2}(E, I)$ was accomplished by taking a series of spectra with the discharge off and $\rho_{2}$ varying much more than any other parameter. Then a linear regression at each value of $E$ separated the signal into a constant part and a coefficient of $\rho_{2}$, which is precisely $\epsilon_{2}(E, I)$. At low electron energies, where the $E$ values are closely spaced, a moving window average can remove some of the noise left by the very large photoelectron component. Figure 14 shows a smoothed $\mathrm{H}_{2}$ spectrum derived by this method from measurements of $12 \mathrm{~J}$ un90 at irradiances near $6 \times 10^{13} \mathrm{~W} / \mathrm{cm}^{2}$. It shows a broad peak at $E=4 \mathrm{eV}$ which can be resolved into three subpeaks by applying a retarding voltage. These represent ionization of $\mathrm{H}_{2}$ to various vibrational states of the $\mathrm{H}_{2}{ }^{+}$ion, possibly involving threephoton resonances with any of several $\mathrm{H}_{2}$ levels. There is a peak near $1 \mathrm{eV}$ from dissociation to $\mathrm{H}$ in the ground state, followed by ionization of the atom. This peak is shifted to lower energies, compared to the usual $S_{0}$ peak, because the atom is dissociated from the molecule in a field that is already strong. The peak near $1.6 \mathrm{eV}$ appears to result from dissociation to $H$ in the $n=2$ state, followed by single-photon ionization of the atom. The features below $1 \mathrm{eV}$ are probably artifacts from subtracting the much larger photoelectron signal in this 


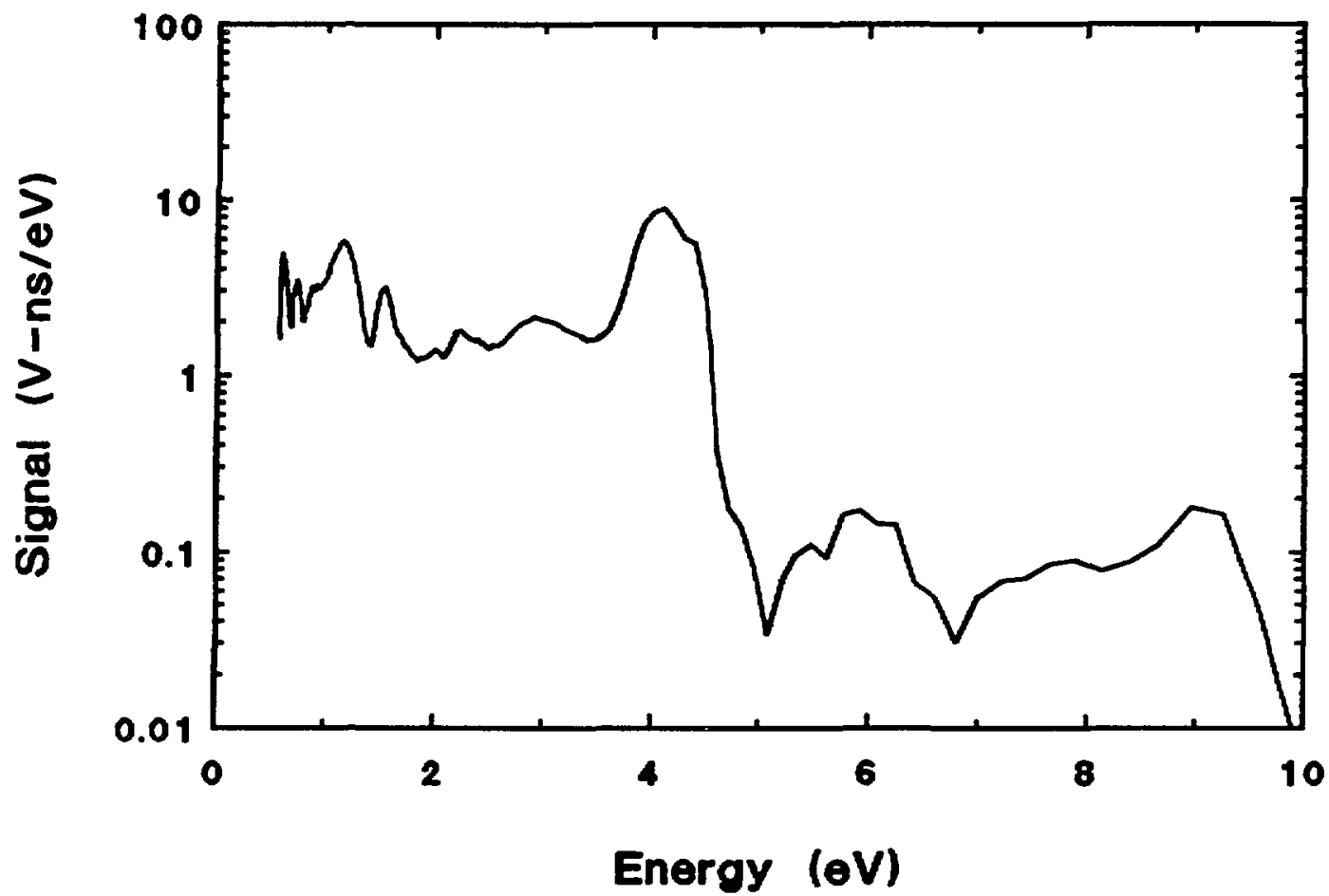

Figure 14. Electron time-of-flight spectrum of molecular hydrogen at an irradiance of $6 \times 10^{13} \mathrm{~W} / \mathrm{cm}^{2}$.

range. A family of $\epsilon_{2}(E, I)$ curves for a range of irradiances near some $I_{0}$ may be represented by the model

$$
\epsilon_{2}(E, I)=\epsilon_{2}\left(E, I_{0}\right)\left(\frac{I}{I_{0}}\right)^{N(E)}
$$

Preliminary calculations from data of 12,20 , and $27 \mathrm{Jun} 90$ suggest that $N(E)$ is between 2 and 3 for energies less than $5 \mathrm{eV}$, and between 3 and 4 for the first set of ATI signals. $N(E)$ may be slightly lower in the continuum region than at the $1 \mathrm{eV}$ and $4 \mathrm{eV}$ peaks. 


\subsection{Space Charge}

In addition to the differences already noted between the curves in Fig. 12, the $S_{0}$ peak is much wider at higher irradiance. In order to interpret the widths of the peaks in terms of ponderomotive potentials, one must first determine whether space charge also contributes to them. Space charge has been a particularly difficult problem in ATI experiments (Crance 1986). Accordingly, I collected a set of electron spectra on 30 ct89 at four different $\mathrm{H}$ densities and three irradiances from $8.3 \times 10^{12} \mathrm{~W} / \mathrm{cm}^{2}$ to $6.7 \times 10^{13} \mathrm{~W} / \mathrm{cm}^{2}$. The presence of space charge was indicated at the higher irradiances by a reduction in peak width with lower $\mathrm{H}$ density. When the density was reduced to the point that the peaks reached a minimum width, however, there was still a component that increased with irradiance, reaching significant fractions of an electron volt. This indicates that the electron energies were shifted by ponderomotive potentials that varied over the focal volume. To keep space charge broadening small compared to the spectrometer's resolution, the total number of electrons produced in the interaction region had to remain below 1000. It should be noted, however, that a broadened peak is still useful when its area, rather than its ponderomotive shift, is of interest.

Table 3 presents the results of measurements on the $S_{0}$ peak. Total pressures in the interaction region, determined from RGA readings, were between 3.9 and $8.7 \times 10^{-8}$ Torr. The discharge could not be sustained at lower pressures. Irradiances were calculated from measured pulse energies, using a waist radius of $35 \mu \mathrm{m}$ and a pulse length of 600 fsec FWHM. The number of electrons produced in the interaction region per laser pulse is calculated from the recorded signal, assuming $7.2 \mathrm{mV}$ average signal per detected electron and 4.3 electrons in the interaction region for each one detected. I have subtracted the portion of the total signal due to photoelectrons from the grids, leaving the ATI signals. The number of electrons produced per pulse was proportional to the total gas pressure, as expected. The width of the $S_{1}$ peak at $6.7 \times 10^{13} \mathrm{~W} / \mathrm{cm}^{2}$ was $0.5 \mathrm{eV}$ 
$(\Delta E / E=0.08)$ at all pressures. This is the width expected from the spectrometer resolution if $\Delta E / E$ is constant over the 1 to $7 \mathrm{eV}$ range. The $S_{1}$ peak was too small to measure accurately at the lower irradiances.

Table 3. Widths (FWHM) of atomic hydrogen $S_{0}$ ATI peak at three irradiances and four atomic densities.

\begin{tabular}{crrrr}
\hline $\begin{array}{r}\text { Irradiance } \\
\left(\mathrm{W} / \mathrm{cm}^{2}\right)\end{array}$ & $\begin{array}{r}\text { Pressure } \\
(\mathrm{nTorr})\end{array}$ & $\begin{array}{r}\text { Electrons per } \\
\text { laser pulse }\end{array}$ & $\begin{array}{r}\text { Electrons per } \\
\text { pulse-nTorr }\end{array}$ & $\begin{array}{r}\text { Width } \\
(\mathrm{eV})\end{array}$ \\
\hline $6.7 \times 10^{13}$ & 39 & 4156 & 107 & 0.25 \\
& 52 & 6245 & 120 & 0.30 \\
& 66 & 8218 & 124 & 0.35 \\
& 87 & 9712 & 112 & 0.39 \\
$2.5 \times 10^{13}$ & & & & \\
& 52 & 888 & 17.1 & 0.15 \\
& 66 & 1168 & 17.7 & 0.15 \\
$8.3 \times 10^{12}$ & 87 & 1693 & 19.5 & 0.17 \\
& 52 & & & \\
& 66 & 47 & 0.9 & 0.08 \\
& 87 & 82 & 1.2 & 0.08 \\
\hline \hline
\end{tabular}

The $S_{0}$ peak widths can be divided into components representing space charge, an intrinsic width, and the resolution of the spectrometer according to the model

$$
\Delta E=\sqrt{W_{s}^{2}+W_{i}^{2}+\left(C_{s} P\right)^{2}}
$$

where $W_{s}=$ spectrometer resolution $=80 \mathrm{meV}$,

$$
\begin{aligned}
W_{i} & =\text { intrinsic width for each irradiance, } \\
C_{s} & =\text { amount of space charge per unit gas pressure, and } \\
P & =\text { total gas pressure. }
\end{aligned}
$$

Derived values of $W_{i}$ and $C_{s}$ are $195 \mathrm{meV}$ and $4 \mathrm{meV} / \mathrm{nTorr}$ at $6.7 \times$ $10^{13} \mathrm{~W} / \mathrm{cm}^{2}$, and $110 \mathrm{meV}$ and $1.1 \mathrm{meV} / \mathrm{nTorr}$ at $2.5 \times 10^{13} \mathrm{~W} / \mathrm{cm}^{2}$. The corresponding values at $8.3 \times 10^{12} \mathrm{~W} / \mathrm{cm}^{2}$ are too small to measure. 


\subsection{Ponderomotive shifts}

The intrinsic widths measured from the $30 \mathrm{ct} 89$ data are consistent with the calculations of Perry et al. (1989). The energy of each electron is shifted by the ponderomotive potential at the point of ionization because the laser pulse is short compared to the time required to leave the focal volume. However, only a few electrons will have their energies shifted by the largest value of ponderomotive potential because of the small volume of maximum intensity. The highest point of the broadened ATI peak indicates the shift experienced by the "average" electron. Perry et al. find that this shift is a fixed fraction $F_{c}$ of the maximum shift, and they calculate that $F_{c}=0.48$ for 3 -photon absorption. One can thus use the shift of the high point, or crest, of the peak to estimate the highest irradiance at which any ionization occurred, but the method has two sources of uncertainty. One source is the value of $F_{c}$. A model of the ionization process (a variation of the HYD program described in the next section) indicates that in the case of this experiment, $F_{c}$ varies from 0.7 at an irradiance of $10^{13} \mathrm{~W} / \mathrm{cm}^{2}$ to 0.37 at the start of depletion. The other source of uncertainty is the energy from which the shift is measured. The crest of an unshifted peak will not be at the energy $E_{K}$ of Eq. 1 (Chapter 1), but will be offset to some energy $E_{K}-$ $\delta E_{K}$ because of the limited resolution of the spectrometer and possibly some differences in contact otentials. The value of $\delta E_{K}$ might be expected to be about half the spectrometer resolution, or $0.04 \mathrm{eV}$. Since the ponderomotive potential is proportional to irradiance, the offset in electron energy produces an offset in calculated irradiance, but the difference between two irradiances depends only on $F_{c}$. The combined uncertainty due to $F_{\mathrm{c}}$ and $\delta E_{K}$ is $\pm 50 \%$. Irradiances calculated from the ponderomotive shifts of 30 ct89 appear in Table 4. The agreement would be better if $F_{c}$ were taken to be smaller than 0.48 , but the lowest-energy electrons that can be identified as belonging to the peak are in fact separated from the highest-energy electrons by about the predicted amount. 
Table 4. Irradiances calculated from ponderomotive shifts in data of 30 ct89. $E_{c}$ is the energy at the $\mathrm{S}_{0}$ crest, with uncertainty $\pm 0.008 \mathrm{eV}$. $\delta E_{K}$ is the offset of an unshifted crest from the nominal energy $E_{K}=$ $1.4 \mathrm{eV} . F_{c}=0.48$. "Known" irradiances are those calculated from pulse energies. Irradiances are in $W / \mathrm{cm}^{2}$.

\begin{tabular}{cccc}
\hline \hline "Known" & $E_{c}(\mathrm{eV})$ & $\delta E_{K}=0.04 \mathrm{eV}$ & $\delta E_{K}=0.09 \mathrm{eV}$ \\
\hline $8.3 \times 10^{12}$ & 1.28 & $2.9 \times 10^{13}$ & $1.1 \times 10^{13}$ \\
$2.5 \times 10^{13}$ & 1.25 & $4.0 \times 10^{13}$ & $2.2 \times 10^{13}$ \\
$6.7 \times 10^{13}$ & 1.17 & $7.0 \times 10^{13}$ & $5.2 \times 10^{13}$ \\
\hline \hline
\end{tabular}

This method produces good results when applied to the spectra collected on 12Jun90, 21Jun90, and 27Jun90 at irradiances, calculated from pulse energies, of $3.3 \times 10^{13} \mathrm{~W} / \mathrm{cm}^{2}, 5.5 \times 10^{13} \mathrm{~W} / \mathrm{cm}^{2}$, and $1.5 \times 10^{14} \mathrm{~W} / \mathrm{cm}^{2}$, respectively. These are shown in Table 5. The discrepancy at the highest irradiance is expected, because depletion had occurred before the time of peak intensity. There were no atoms left to experience the peak ponderomotive potential. Perry et al. use the value of irradiance $I_{\text {sat }}$ at which $E_{c}$ ceases to increase in an estimate of the generalized cross section of the ionization process $\sigma^{(K)}(\omega)$ from the relation

$$
\sigma^{(K)}(\omega) I_{\text {sat }}^{K} T_{p}^{(K)}=1
$$

where

$$
T_{p}^{(K)}=2^{K-1} \frac{(K-1) !}{(2 K-1) ! !} T_{p},
$$

$T_{p}$ is the pulse length used previously, and $K$ is the order of the process. When $K=3, T_{p}=700 \mathrm{fsec}$, and $I_{\text {sat }}=10^{14} \mathrm{~W} / \mathrm{cm}^{2}$,

$$
\sigma^{(K)}(\omega)=1.2 \times 10^{-84} \mathrm{~cm}^{6} \mathrm{~s}^{2}, \text { or } 1.9 \times 10^{-48} \mathrm{~cm}^{6} / \mathrm{W}^{2},
$$

depending on whether the generalized cross section is used with three irradiance terms in units of photon flux, or only one in photon flux and the others in W/cm${ }^{2}$. This value is close to Perry's measurement in xenon at $293 \mathrm{~nm}$, but much lower than the LOPT value of $3 \times 10^{-46} \mathrm{~cm}^{6} / \mathrm{W}^{2}$. 
Table 5. Irradiances calculated from ponderomotive shifts in data of 12, 21, and 27Jun90. Entries are as in Table 4.

\begin{tabular}{ccc}
\hline \hline "Known" & $E_{\varepsilon}(\mathrm{eV})$ & $\delta E_{K}=0.04 \mathrm{eV}$ \\
\hline $3.3 \times 10^{13} \mathrm{~W} / \mathrm{cm}^{2}$ & 1.29 & $2.5 \times 10^{13} \mathrm{~W} / \mathrm{cm}^{2}$ \\
$5.5 \times 10^{13} \mathrm{~W} / \mathrm{cm}^{2}$ & 1.21 & $5.5 \times 10^{13} \mathrm{~W} / \mathrm{cm}^{2}$ \\
$1.5 \times 10^{14} \mathrm{~W} / \mathrm{cm}^{2}$ & 1.11 & $9.1 \times 10^{13} \mathrm{~W} / \mathrm{cm}^{2}$ \\
\hline \hline
\end{tabular}

\subsection{Comparison of measurements to theoretical predictions}

The theoretical predictions that have been presented in Fig. 4 may be recast in the form of numbers of electrons produced in the interaction region at various values of the peak irradiance for comparison to measured electron counts. The measurements must similarly be traced back to the interaction region, considering the system gain $G_{S}$ (Eq. 36 of Chapter 3) and the atomic density in the interaction region. Since the measured ATI peaks decrease in area from $S_{0}$ to $S_{1}$ by factors on the order of 40 (see Table 2), the area of the $S_{0}$ peak can be used to represent the total rate of MPI within the uncertainties of the data. Figure 15 shows such a comparison, normalized to a density of $10^{10}$ atoms $\mathrm{cm}^{-3}$. As in Fig. 4, the highest curve is LOPT, the second highest is Floquet theory, the middle curve is the Coulomb-corrected Keldysh model, and the lowest two are the Keldysh and Reiss models without Coulomb correction.

The procedure for folding the theoretical calculations with the experimental parameters to predict the number of ionizations per laser pulse is similar to that of Perry, Landen, and Szöke (1989). It was implemented in a computer program called HYD written by Dr. George Kyrala. The program first divides the focal volume into shells along contours of equal peak irradiance, so that all atoms within a shell labeled by the subscript $p$ experience the same temporal history 


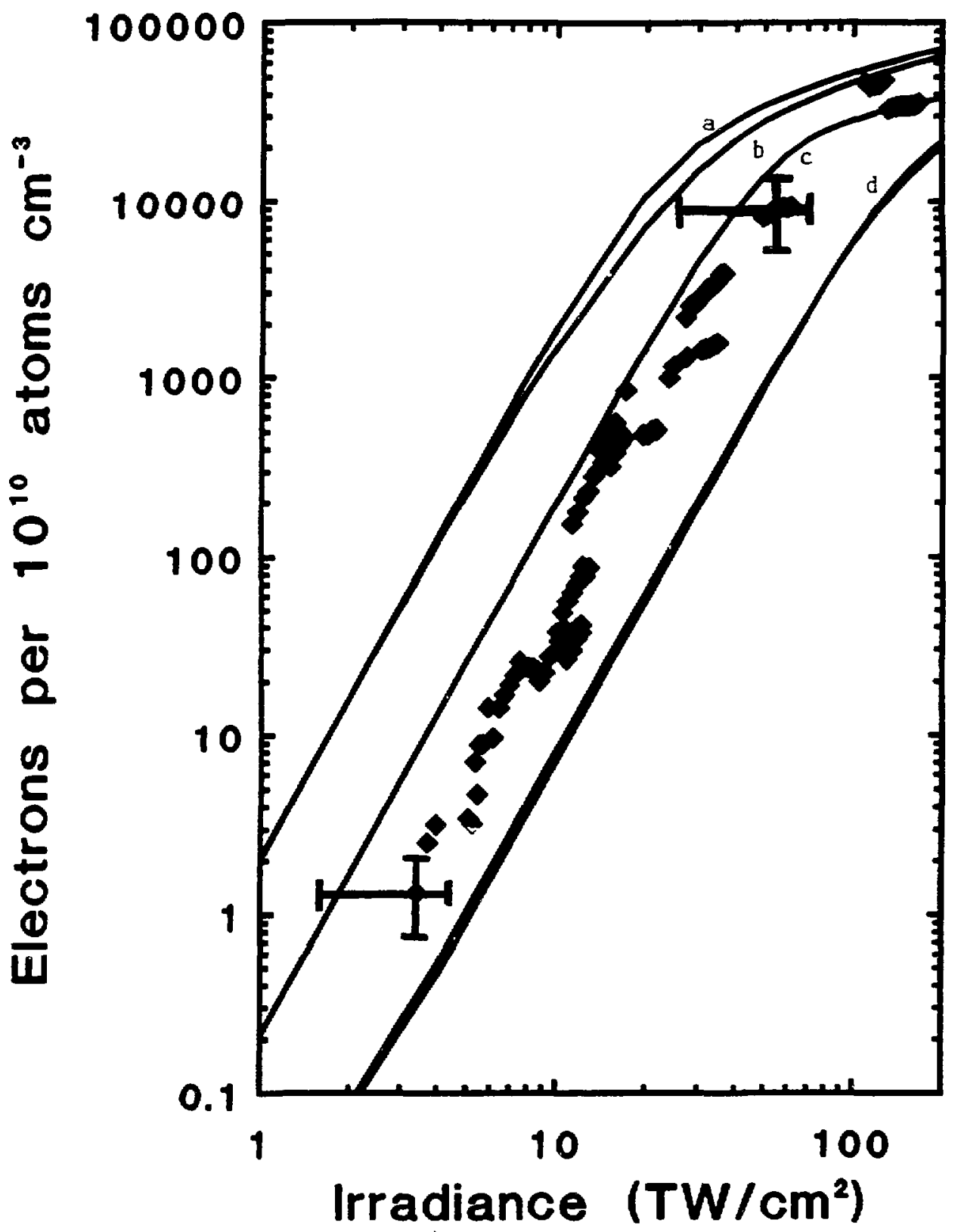

Figure 15. Comparison of measured and predicted total numbers of electrons produced in the interaction region by MPI of atomic hydrogen, using the smallest measured waist radius $W_{0}=35 \mu \mathrm{m}$. Each curve is normalized to an atomic density of $10^{10}$ atoms $\mathrm{cm}^{-3}$. The data are shown as unconnected points. Curve (a) is LOPT evaluated by Khristenko and Vetchinkin (1976). Curve (b) is the Floquet theory of Chu (1990). Curve (c) is the Coulomb-corrected Keldysh model (1965). Curves (d) are the Keldysh (1965) and Reiss (1980) models without Coulomb correction. 
$I_{p} f(t)$, with $f(t)$ the same for all shells. The program assumes the temporal distribution

$$
f(t)=\sin ^{2}\left(\pi t / T_{1}\right)
$$

where $T_{l}$ is chosen to fit the pulse length of the laser. Thus the irradiance is zero at times 0 and $T_{l}$, and is $I_{p}$ at the peak. Within the innermost shell, the peak irradiance is $I_{0}$. Use of this $f(t)$ rather than the more accurate $\operatorname{sech}^{2}\left(2 t / T_{p}\right)$ allowed the program to take fewer time steps. Test calculations with $\operatorname{sech}^{2}\left(t / T_{p}\right)$ showed that the final results were the same when $T_{1}$ was chosen to be $1.37 \mathrm{ps}$. The volume of each shell is obtained by inverting Eq. 32 to find the coordinates of an irradiance contour according to the $M^{2}$ model, then integrating over irradiance. The total volume enclosed by the shell of peak irradiance $I_{p}$ is

$$
V\left(I_{p}, W_{0}, M^{2}\right)=\frac{\pi^{2} W_{0}^{4}}{M^{2} \lambda} V^{*}(\zeta, \xi)
$$

where

$$
\begin{aligned}
V^{*}(\zeta, \xi) & =V_{1}^{*}+V_{2}^{*}, \\
V_{1}^{*} & =\left(\zeta+\frac{\zeta^{3}}{3 !}\right) \ln \left(\frac{\xi}{\zeta^{2}}\right), \\
V_{2}^{*} & =\frac{2}{9} \zeta^{3}+\frac{4}{3} \zeta-\frac{4}{3} \tan ^{-1} \zeta,
\end{aligned}
$$

and $\zeta$ and $\xi$ are the limits of integration in non-dimensional axial and radial coordinates. If the shell is contained wholly within the atomic beam,

$$
\xi=I_{0} / I_{p}, \quad \zeta=\sqrt{\xi-1} \quad \text { (no truncation) }
$$

If the shell extends beyond the limits of the atomic beam at $z= \pm z_{H}$, it is truncated by setting

$$
\zeta=\frac{i_{H} M^{2} \lambda}{\pi W_{0}^{2}} \quad \text { (truncated) }
$$

The volume $V_{p}$ of the shell is then the difference between the total volume it encloses and the total volume enclosed by the next smaller shell. $V_{p}$ is multiplied by a hydrogen density of $10^{10}$ atoms $\mathrm{cm}^{-3}$ to obtain the number of atoms in the 
shell at the start of the pulse $N_{h}\left(I_{p}, 0\right)$. The number of electrons produced up to a time $t$ is thus

$$
N_{e}\left(I_{p}, t\right)=N_{h}\left(I_{p}, 0\right)\left[1-\exp \left(-\int_{0}^{t} W_{K}\left(I_{p} f\left(t^{\prime}\right)\right) d t^{\prime}\right)\right]
$$

where $W_{K}(I)$ is the transition rate for $K$ photons at irradiance $I$. The total number of electrons produced by the pulse is then the sum over all the shells after the pulse has ended, approximating the integral

$$
N_{e}\left(I_{0}\right)=\int_{0}^{I_{0}} N_{e}\left(I_{p}, T_{l}\right) \frac{d V\left(I_{p}, W_{0}, M^{2}\right)}{d I_{p}} d I_{p}
$$

A significant feature of the final result is its dependence on the parameters of the laser beam. In the $M^{2}$ model, $M^{2}$ and $W_{0}$ are not independent, but are proportional to each other for a given optical system. Therefore, in the absence of truncation, the electron production rate at fixed $I_{0}$ varies as

$$
N_{e}\left(I_{0}\right) \propto W_{0}^{3} \quad \text { (no truncation) }
$$

In this experiment, however, truncation occurs whenever $I_{0}$ is large enough to produce any noticeable number of electrons. The interaction volume is effectively a cylinder of radius $W_{0}$ and length $2 z_{H}$ regardless of $M^{2}$. In the calculation, $\zeta$, which depends on $M^{2}$ and $W_{0}$, causes $V^{*}(\zeta, \xi)$ to vary as $M^{2} / W_{0}^{2}$, so that

$$
N_{e}\left(I_{0}\right) \propto W_{0}^{2} \quad \text { (truncated) }
$$

Of course, in either case, the pulse energy must grow as $W_{0}^{2}$ to hold $I_{0}$ constant.

The data in Fig. 15 tend to cluster together, leaving gaps in the curve. We believe this represents occasional variations in the waist size of the laser focus and possibly also in other parameters which were not measured on a routine basis (see Section 4.2). Other than these shifts, which can amount to factors as large as ten in electron count, or, equivalently, factors of two in irradiance in the steep portion of the curve, the uncertainty in measuring peak irradiance values was 
primarily that of defining the waist area, or $\pm 12 \%$. (Recall from Section 3.3.1 that

$$
\left.I_{0} \propto W_{0}^{-2}, \text { and } \frac{\Delta W_{0}}{W_{0}}=0.06 .\right)
$$

The horizontal error bar in Fig. 15 represents the combined uncertainty in calculating $I_{0}$ from the waist radius, the length of the pulse, and the energy in the pulse. Table 6 presents the individual factors that contributed to the total uncertainty. Each factor represents a conservative estimate; for example, the uncertainty in the measurement of $W_{0}$ was estimated (in Appendix C) from the 90 th percentile of $\chi^{2}$, which corresponds to 2.3 standard deviations of a normally distributed variable. In addition, all of the maximum (minimum) values of the factors are multiplied to obtain the maximum (minimum) overall factor. This is the worst-case assumption that all of the systematic errors conspire to maximize the total error. Table 6 also shows the total error under the assumption that the individual errors add in quadrature. The error bar for Fig. 15 includes the possibility that the waist radius was near $45 \mu \mathrm{m}$ instead of near $35 \mu \mathrm{m}$ when the data were collected. This is why it extends much farther in the direction of lower irradiances (larger waist radii). There is a possibility of additional error in the low and middle irradiance values because pairs of filters were often used to obtain small steps of attenuation, whereas the highest irradiances were obtained with single filters or with beamsplitters. The irregular surfaces of these filters may have increased the waist radius more when used in pairs than when used singly - one filter with particularly flat surfaces repeatedly produced six times as many electrons as a pair with the same total density. That filter was not used to collect the data in Fig. 15, however, and those data showed no systematic differences between single filters and pairs of filters. Replacing the filters with two additional beamsplitters did not solve the problem completely, either, because these surfaces, while much flatter than those of the filters, were still $\lambda / 4$ for UV 
light. Therefore, we estimate a single uncertainty in irradiance for all of the data in Fig. 15.

The uncertainty in electron count comes mostly from the detection efficiency factor in $G_{S}$ (Appendix D) and the factor that normalizes the count to a gas density of $10^{10}$ atoms $\mathrm{cm}^{-3}$. The calculation of the vertical error bar is summarized in Table 6, where the tota! uncertainty shown in the figure again represents cases in which all the systematic errors are in the same direction. The uncertainty in measuring the atomic density is not well-characterized, but we estimate it as $\pm 20 \%$. There is also an uncertainty in the electron count due to the statistical nature of the ionization process, especially after subtracting a background count with its own statistical noise, but this uncertainty was reduced to $6 \%$ or less of the $S_{0}$ area by averaging hundreds or thousands of individual spectra. It became less than $1 \%$ at high irradiances, where the real $S_{0}$ signal was far greater than the background across most of the peak. It was fortunate that the low-irradiance peaks, though smaller, were better separated from the photoelectron noise. If they had been as wide as the high-irradiance peaks, the background subtraction would have produced error bars on the order of $100 \%$. Since one of these statistical factors increases as the other decreases with irradiance, and since both were small compared to the uncertainties in gas density and detection efficiency, we again estimate a single uncertainty for all of the points in Fig. 15.

Finally, areas of the $S_{1}$ peaks corresponding to the data in Fig. 15 appear in Fig. 16, along with some $S_{2}$ areas that could be identified. These data cannot readily be compared to theoretical predictions, since the small numbers of electrons in the higher peaks would have required very long collections (several hours) to accumulate adequate average signals, and the experimental conditions frequently changed significantly over the course of a day. Further, comparisons to theoretical "branching ratios" among the ATI peaks are generally performed over large numbers of peaks to define a trend, and the high photon energy in this 
Table 6. Uncertainties in measured electron counts and irradiances, including the normalization to a specified gas density. Each individual uncertainty is represented by the highest and lowest factors by which the "best" value might be multiplied. The "worst-case" uncertainty, assuming all systematic errors conspire to increase the total error, is obtained by multiplying the individual factors together. This is the uncertainty plotted in Fig. 15 and 17. The "optimistic" uncertainty assumes that the errors add in quadrature.

\begin{tabular}{lcc}
\hline \hline \multicolumn{2}{l}{ Uncertainty in normalized electron count } & \\
\hline Source of uncertainty & Minimum factor & Maximum factor \\
\hline Efficiency of electron detector & 0.8 & 1.2 \\
Normalization to standard density & 0.8 & 1.2 \\
Gain of electron detector & 0.94 & 1.06 \\
Statistical noise & 0.94 & 1.06 \\
"Worst-case" uncertainty & 0.57 & 1.62 \\
"Optimistic" uncertainty & 0.70 & 1.30 \\
\hline \hline Uncertainty in irradiance for $W_{0}=35 \mu \mathrm{m}$ & \\
\hline Source of uncertainty & Minimum factor & Maximum factor \\
\hline Change of waist radius & 0.60 & 1. \\
Measurement of waist radius & 0.88 & 1.12 \\
Measurement of pulse length & 0.92 & 1.08 \\
Calibration of joulemeter & 0.93 & 1.07 \\
"Worst-case" uncertainty & 0.46 & 1.30 \\
"Optimistic" uncertainty & 0.57 & 1.16 \\
\hline \hline Uncertainty in irradiance for $W_{0}=45 \mu \mathrm{m}$ & \\
\hline Source of uncertainty & Minimum factor & Maximum factor \\
\hline Change of waist radius & 1. & 1.65 \\
Measurement of waist radius & 0.88 & 1.12 \\
Measurement of pulse length & 0.92 & 1.08 \\
Calibration of joulemeter & 0.93 & 1.07 \\
"Worst-case" uncertainty & 0.75 & 2.14 \\
"Optimistic" uncertainty & 0.84 & 1.67 \\
\hline \hline
\end{tabular}


experiment caused the number of peaks to be low. It does appear, however, that the higher ATI peaks increase in size more quickly with irradiance than the $S_{0}$ peak does, and the branching ratio between $S_{0}$ and $S_{1}$ is of the order of 40 in the $5 \times 10^{13} \mathrm{~W} / \mathrm{cm}^{2}$ range of irradiances. Solid lines in the figure indicate the slopes to be expected for indices of nonlinearity of 3,4 , and 5 for the $S_{0}, S_{1}$, and $S_{2}$ data respectively. While the $S_{2}$ points suffer from large statistical uncertainties due to count rates as low as one per 100 laser shots, one might suspect from Fig. 16 that their index of nonlinearity is less than five.

\subsection{Conclusions}

The data in Fig. 15 fall between the highest and the lowest theoretical predictions, differing from each extreme by a large factor. The data and the extreme models cannot be brought together by assuming that a single measurement was in error without greatly exceeding the estimated uncertainties. Let us consider what adjustments might be required. The single parameter that requires the least adjustment, in proportion to its confidence interval, to match the extreme models is the waist radius $W_{0}$, since an increase there reduces the calculated peak irradiance as $W_{0}^{-2}$ and increases the predicted electron count by the same factor. Thus the data points shift to the left and downward by equal distances, relative to the prediction curves, moving at an angle to the $I^{3}$ slope of the curves. Figure 17 shows the effect of increasing $W_{0}$ from $35 \mu \mathrm{m}$ to $45 \mu \mathrm{m}$. The vertical error bar is the same as in Fig. 15, while the horizontal error bar is reversed, since the assumed waist radius is now the largest that was measured. This $30 \%$ increase in $W_{0}$ is just enough to bring the data into agreement with the Coulomb-corrected Keldysh model, which is the closest model in Fig. 15. Similar agreement could be obtained in the steep portion of the curve by reducing the assumed gas density or the assumed collection efficiency of the electron spectrometer by a factor of 2.8 , but errors of this size seem far less likely. Alternatively, the calculated irradiance might be reduced by a factor of 1.4 with no change in $W_{0}$ by increasing 


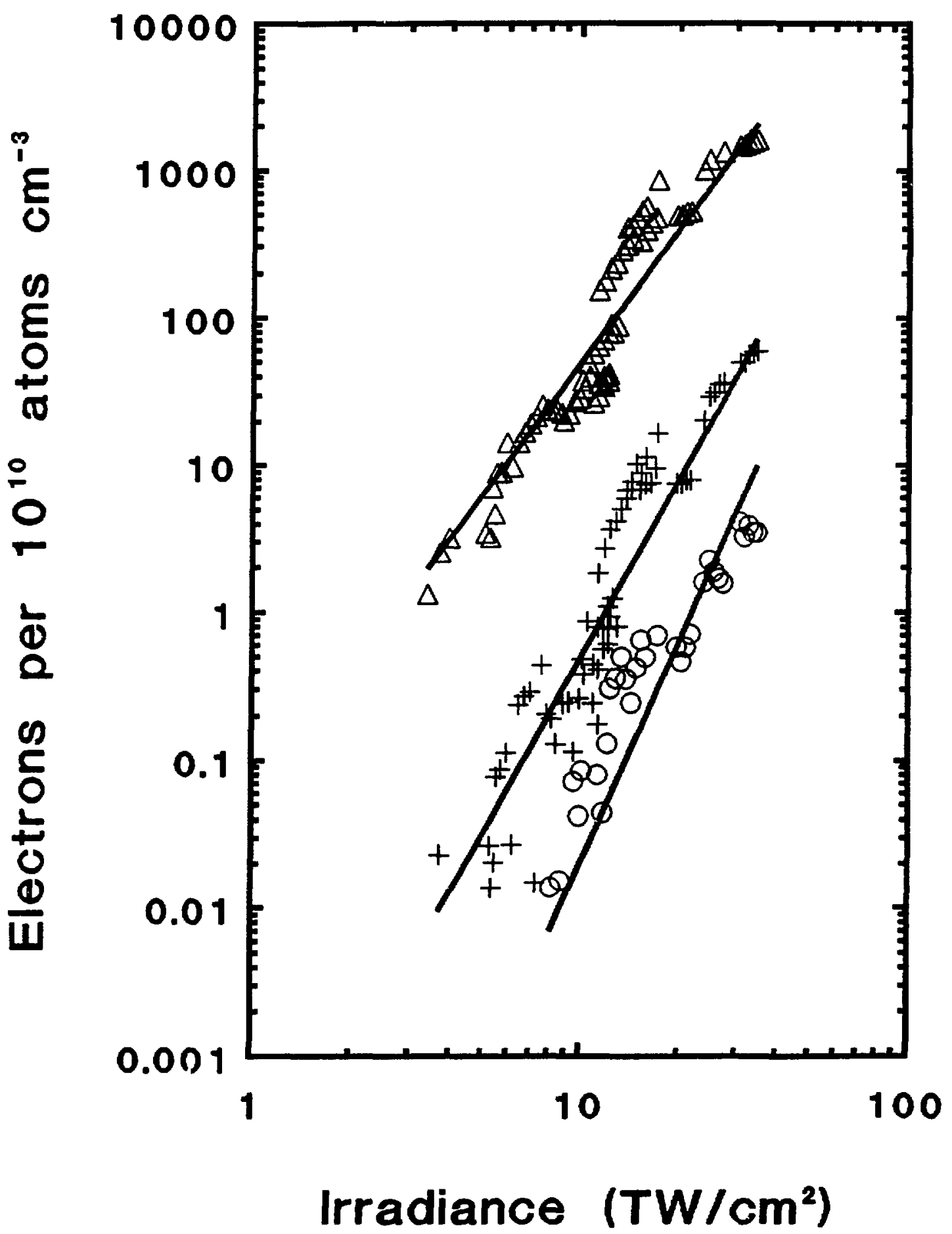

Figure 16. Data of Fig. $15(\Delta)$ compared to areas of the $S_{1}(+)$ and $S_{2}$ (O) ATI peaks in the same electron spectra. The lines through the data sets go as $I^{3}, I^{4}$, and $I^{5}$ respectively. They are not fit to the data. 
the assumed pulse length or the joulemeter calibration factor, but the change required is again much larger than the estimated uncertainties in these parameters. Much larger adjustments are required to match the perturbative results $\left(W_{0} \approx 75 \mu \mathrm{m}\right)$ or the Reiss-Keldysh curves $\left(W_{0} \approx 20 \mu \mathrm{m}\right)$. Thus we conclude that the data stand in disagreement with the predictions of lowest order perturbation theory, Floquet theory, and KFR theory with pure Volkov final states. Calculations of such effects as plasma heating by ATI could be seriously in error if these models are used. The Coulomb-corrected Keldysh model is in better agreement with the data. We have no comparison to the results of numerical integration of the Schrödinger equation, because these have not been extended to low enough irradiances.

The rates of production of ATI $\left(S_{1}\right.$ and $\left.S_{2}\right)$ electrons are much smaller than those of $S_{0}$ electrons. The rates decrease by about a factor of 40 from one peak to the next in the $5 \times 10^{13} \mathrm{~W} / \mathrm{cm}^{2}$ range of irradiances. This agrees with the general observation that the number of ATI peaks increases with the wavelength of the laser. Because the number of electrons in each ATI peak is small, the areas of the ATI peaks carry large statistical uncertainties as well as uncertainties of calibration. It is therefore impossible to determine precise indices of nonlinearity for the ATI peaks, but they appear to be higher than that of the $S_{1}$ peak, as generally expected.

Depletion of the atoms in the interaction region limited the irradiance at ionization to $10^{14} \mathrm{~W} / \mathrm{cm}^{2}$ or less. This means that lasers with much shorter rise times, on the order of femtoseconds, will be needed to explore ATI by UV fields at irradiances for which the ponderomotive potential exceeds the photon energy. 


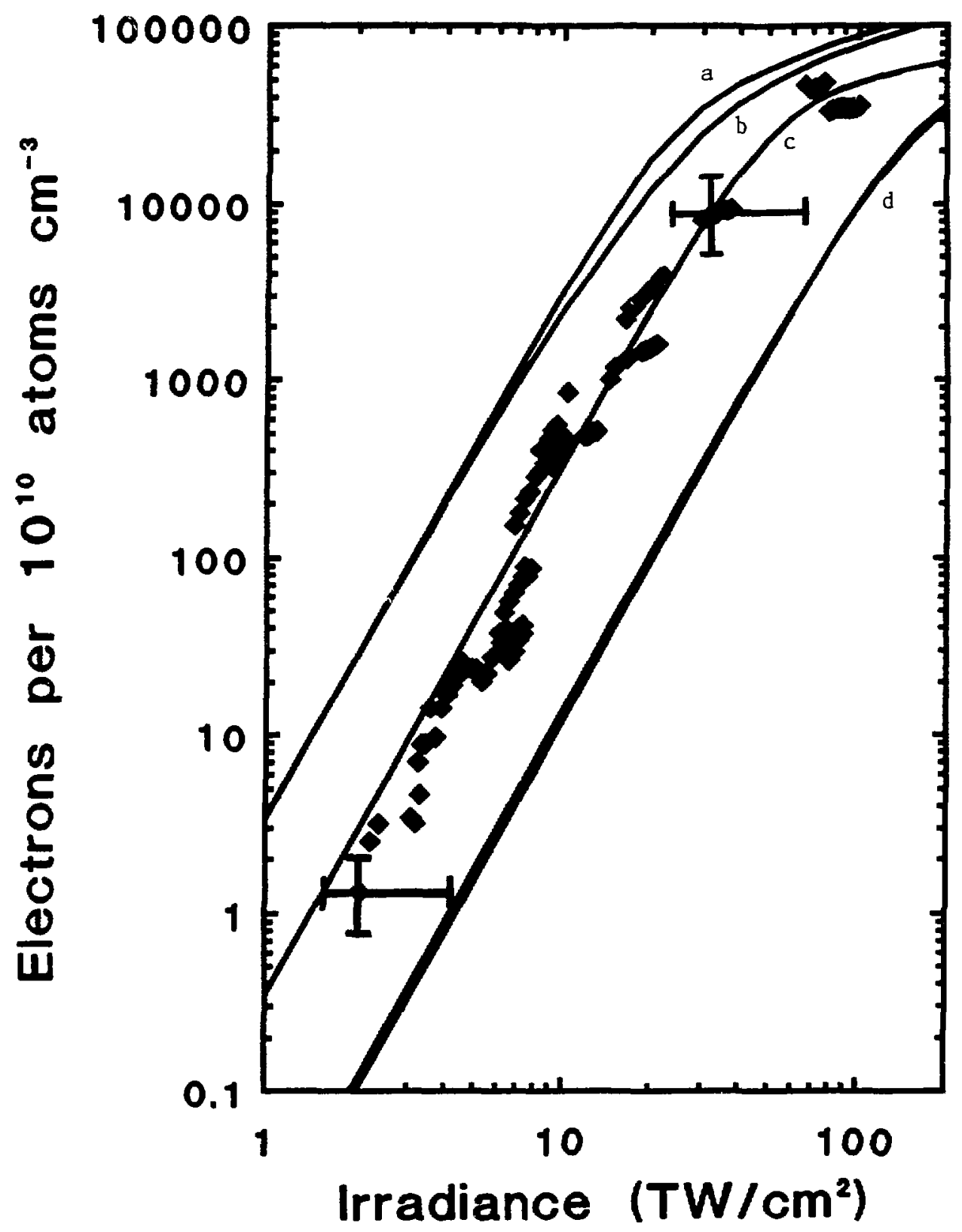

Figure 17. Comparison of measured and predicted total numbers of electrons produced in the interaction region by MPI of atomic hydrogen, using the largest measured waist radius $W_{0}=45 \mu \mathrm{m}$. The data are shown as unconnected points. Curve (a) is LOPT evaluated by Khristenko and Vetchinkin (1976). Curve (b) is the Floquet theory of Chu (1990). Curve (c) is the Coulomb-corrected Keldysh model (1965). Curves (d) are the Keldysh (1965) and Reiss (1980) models without Coulomb correction. 


\subsection{Suggestions for further work}

Other experiments have confirmed theoretical predictions that circularly polarized light produces lower ionization rates and different distributions of electrons among the ATI peaks. Circular polarization would also remove the last trace of two-photon resonance with the $2 \mathrm{~s}$ state at low irradiance. Addition of a quarter-wave plate to the optical system could provide any desired ellipticity for such measurements with the present apparatus. The ellipticity would have to be measured as the beam left the apparatus to account for unequal reflectivities of the mirrors for the different polarization components, but this would be only a minor difficulty. Further experiments at all polarizations could also be done with the strong red pulse that is produced within the front end of the LABS-I laser system.

Since $\mathrm{H}_{2}$ spectra were unavoidably collected, they should be compared to theories of molecular ATI. Similar experiments have had interesting results (Luk and Rhodes 1988, Verschuur et al. 1989).

The positive helium ion is like a hydrogen atom with a $54 \mathrm{eV}$ ionization potential in photoionization. It would he an interesting target if it could be prepared at high enough densities. It could be separated electrically from the neutral atom background, and there would be no molecular background. A low target density might then be tolerable from the viewpoint of signal to noise, and it would avoid all space charge problems. The combination of a high photon energy with a high ionization potential would test whether the photon energy itself or its size relative to the binding energy determines the number of ATI peaks produced. 


\section{Appendix A. Units, magnitudes, and dimensionless parameters}

Three systems of units regularly appear in the ATI literature. One is the system of atomic units (a.u.) introduced by Hartree (1928) and summarized by Bethe and Salpeter (1957). These are convenient in theoretical papers because the notation is simple and many numerical values are of order one. Other authors use natural units, in which $\hbar=c=1$, but $m$, e, etc. have their cgs values. This choicc facilitates checking the results of a calculation for correct dimensions. Experimental papers generally use a mix of cgs and SI units. Irradiances, for example, are expressed in $\mathrm{W} / \mathrm{cm}^{2}$. In addition, pressures are usually given in torrs, where

$$
0.75 \text { Torr }=1 \mathrm{mbar}=100 \mathrm{~Pa} .
$$

One purpose of this appendix is to relate the different units used in this dissertation to each other. The other is to place the experiment in perspective by evaluating several parameters that are commonly used to indicate what processes are important and what approximations are useful at any combination of field strengths and wavelengths.

Table 7. Atomic units related to cgs and other commonly used units. The fundamental atomic units are $m, e$, and $\hbar$.

\begin{tabular}{rccl}
\hline \hline Unit of & Symbol & Formula in cgs & Value in other units \\
\hline length & $a$ & $\hbar^{2} / m e^{2}$ & $5.3 \times 10^{-9} \mathrm{~cm}$ \\
velocity & $v_{0}$ & $e^{2} / \hbar$ & $2.2 \times 10^{8} \mathrm{~cm} / \mathrm{sec}$ \\
energy & $e^{2} / a$ & $m e^{4} / \hbar^{2}$ & $27.21 \mathrm{eV}$ \\
time & $a / v_{0}$ & $\hbar^{3} / m e^{2}$ & $2.4 \times 10^{-17} \mathrm{sec}$ \\
frequency & $v_{0} / a$ & $m e^{2} / \hbar^{3}$ & $4.1 \times 10^{16} \mathrm{~Hz}$ \\
electric potential & $e / a$ & $m e^{3} / \hbar^{2}$ & $27.21 \mathrm{~V}$ \\
electric field strength & $e / a^{2}$ & $m^{2} e^{5} / \hbar^{4}$ & $5.1 \times 10^{9} \mathrm{~V} / \mathrm{cm}$ \\
\hline \hline
\end{tabular}


In atomic units, the charge of the electron $e$, the mass of the electron $m$, and Planck's constant $\hbar$ are all unity. Other units are derived from them. This results in the radius of the first Bohr orbit being 1 a.u., the velocity of an electron in the first Bohr orbit being 1 a.u., and the ionization potential of hydrogen being 0.5 a.u. (if the nucleus were infinitely massive). Table 7 presents the basic atomic units, equivalent expressions in cgs units, and their values in units commonly used in experimental papers. The speed of light $c$ in a.u. is not unity, but the inverse of the fine structure constant $\alpha$, so that

$$
\alpha=\frac{e^{2}}{\hbar c}=\frac{1}{137}
$$

The atomic unit of root mean square (rms) irradiance, corresponding to unit peak electric field strength, is

$$
I_{a}=3.54 \times 10^{16} \mathrm{~W} / \mathrm{cm}^{2}
$$

Thus an electric field $E(t)=E \cos (\omega t)$ with $E=0.1$ a.u. has an rms field strength of $E / \sqrt{2}=0.071$ a.u. and rms irradiance 0.01 a.u. $=3.54 \times 10^{14} \mathrm{~W} / \mathrm{cm}^{2}$. The frequency of a $\mathrm{KrF}$ laser, and hence the photon energy, is 0.184 a.u.

Many dimensionless parameters are used in the ATI literature. In the following discussion, parameters are defined in atomic units, with electric fields $E(t)=E \cos (\omega t)$. Values of the parameters in other units are also given. In these expressions, $I$ is irradiance in $\mathrm{W} / \mathrm{cm}^{2}$, and $\omega=0.184$. Recall that $N_{0}$ is the minimum number of photons required to supply the binding energy $E_{B}$. Hence $N_{0}=3$, while $E_{B}=13.6 \mathrm{eV}$.

Many of the dimensionless parameters may conveniently be defined in terms of the ponderomotive potential $U$, which has dimensions of energy. $U$ is not a true potential, as discussed in Chapter 2, but the kinetic energy of an electron in a laser field, averaged over a cycle of the field:

$$
U=\frac{E^{2}}{4 \omega^{2}}
$$




$$
U(\mathrm{eV})=5.72 \times 10^{-15} I\left(\mathrm{~W} / \mathrm{cm}^{2}\right) \text { for } \mathrm{KrF} .
$$

A free electron in the field must have an energy at least equal to the ponderomotive potential. At irradiances for which $E_{B}+U>N_{0} \omega$, for example, the $S_{0}$ ATI channel is closed. The greatest value of $U$ in the present work is not high enough to close the $S_{0}$ channel.

Another dimensioned quantity of importance is the quiver amplitude $\alpha_{q}$, also known as the Kramers translation length:

$$
\begin{gathered}
\alpha_{q}=E / \omega^{2} \\
\alpha_{q}(\text { Bohr radii })=1.57 \times 10^{-7}\left(I\left(\mathrm{~W} / \mathrm{cm}^{2}\right)\right)^{1 / 2} \text { for } \mathrm{KrF} .
\end{gathered}
$$

When $I=2 \times 10^{14} \mathrm{~W} / \mathrm{cm}^{2}, \alpha_{q}=2.2 \mathrm{Bohr}$ radii, which means that the potential energy of a classical ionizing electron is varying by large amounts compared to its ground state value. This may cause calculations involving approximations to the potential to fail.

The most important measure of field strength according to many theories (Reiss 1980, Becker 1987, Javanainen and Eberly 1988) is the dimensionless parameter

$$
\eta=\frac{U}{\omega}=\frac{E^{2}}{4 \omega^{3}}=1.15 \times 10^{-15} I\left(\mathrm{~W} / \mathrm{cm}^{2}\right) \text { for } \mathrm{KrF} .
$$

ATI becomes important when $\eta$ approaches one. Our highest value of $\eta$ was 0.23 , but the supply of atoms was depleted about the time $\eta$ reached 0.1 . The $\omega^{-3}$ frequency dependence explains why $\mathrm{CO}_{2}$ and $\mathrm{Nd}$ lasers achieve ATI with lower values of $I$ than $\mathrm{KrF}$ lasers do.

Keldysh (1965) defined a parameter $\gamma$ to judge whether the ionization process resembles dc tunneling $(\gamma \ll 1)$ or weak field multiphoton ionization $(\gamma \gg 1)$. Its definition is

$$
\frac{1}{\gamma^{2}}=\frac{2 U}{E_{B}}
$$


The present experiment, with $\gamma>3.7$ prior to depletion, did not enter the tunneling regime.

All of the predicted ionization rates in this work are derived from nonrelativistic modeis. That is, they assume $v / c<0.1$, where

$$
\frac{v}{c}=\frac{E}{c \omega}=\sqrt{\frac{4 U}{c^{2}}}
$$

Our value at depletion was $v / c=0.002$.

A common use of the above parameters is to estimate the irradiance for which LOPT begins to fail. Perturbative calculations are suspect whenever any of these conditions is violated. Faisal (1987) sets a condition of $U<1$. Beyond this, the higher order terms cannot be expected to be smaller than the lower order terms. Potvliege and Shakeshaft (1989b) state the same condition by saying that the quiver speed $v_{q}$ of a free electron, which is

$$
v_{q}=E / \omega=\sqrt{4 U}
$$

must be less than the orbital speed of a bound electron (1 a.u.). The latter paper also specifies $\alpha_{q}<1$ a.u. and $\eta<1$. 


\section{Appendix B. Calculations concerning the vacuum system}

\section{Sensitivity of the RGA to atomic and molecular hydrogen}

RGA sensitivity to a gas is the product of three factors: ionization cross section, quadrupole transmissivity, and electron multiplier gain. In general, the first two vary in opposite directions with molecular mass, so that their product is more or less constant. In the case of $\mathrm{H}$ and $\mathrm{H}_{2}$, however, there is a considerable difference. The third factor generally decreases with mass, but it can be eliminated by using the Faraday cup detector instead of the eleciron multiplier. This section shows how the overall sensitivities to $\mathrm{H}$ and $\mathrm{H}_{2}$ were determined and how the density of $\mathrm{H}$ atoms was calculated from the RGA readings.

The sensitivity to any gas can appear to vary if the instrument is improperly adjusted or improperly operated. The quadrupole voltages must be set at the peak of the response curve each time a particular mass is sampled, and the peak must not move. When the RGA was new, the mass-2 peak had several deep nulls, which appeared at different places at different gas pressures and caused erratic readings. The manufacturer then replaced the sensor head and readjusted the electronics. Afterward, the nulls were much less deep, and readings taken at the nominal positions of the peaks were reliable. Curiously, the option in the computer program that searched near each nominal position for the true peak became entirely useless.

It must be remembered that the total signal due to a particular molecule may be divided among several channels of output. In addition to the mass1 reading due to $H$, which might be called the "diagonal" signal, there is an "off-diagonal" mass-1 signal due to $\mathrm{H}_{2}$. Similarly, there are mass-1 and mass-2 signals due to $\mathrm{H}_{2} \mathrm{O}$. These result from dissociation of the original molecules in the 
process of ionization. In principle, there is a mass-2 signal from recombination of atoms within the RGA, but it is negligible in similar RGA's (Chan et al. 1988), and it is not very important in determining the one quantity needed to interpret the experiment, which is the density of H. Extra signals from double ionization without dissociation also occur, but not in the mass- 1 and mass- 2 channels. The relation between the indicated pressure $P_{i}$ at mass $i$ and the densities of $\mathrm{H}\left(\rho_{1}\right)$ and $\mathrm{H}_{2}\left(\rho_{2}\right)$ at the sensor head may be represented by a matrix $\alpha_{i}$, so that

$$
\begin{aligned}
& P_{1}=\alpha_{11} \rho_{1}+\alpha_{12} \rho_{2} \\
& P_{2}=\alpha_{21} \rho_{1}+\alpha_{22} \rho_{2}
\end{aligned}
$$

Here we ignore the influence of other gases because their densities were at most equal to that of $\mathrm{H}_{2}$ during data collection and the off-diagonal coefficients are small.

The atomic density $\rho_{1}$ is derived from the change in $P_{2}$ when the discharge comes on, with the assumptions that the change in $\rho_{1}$ is

$$
\begin{aligned}
\Delta \rho_{1} & =-2 \Delta \rho_{2}, \text { and that } \\
\rho_{1} & =0 \text { before the discharge starts. }
\end{aligned}
$$

Then

$$
\begin{gathered}
\Delta P_{2}=\left(\alpha_{21} \rho_{1}-\frac{1}{2} \alpha_{22}\right) \rho_{1}, \text { and } \\
\rho_{1}=\Delta \rho_{1}=\frac{-2 \Delta P_{2}}{\alpha_{22}-2 \alpha_{21}} \approx \frac{-2 \Delta P_{2}}{\alpha_{22}} .
\end{gathered}
$$

Since the RGA reading $P_{2}$ agreed with the ion gauge reading at pressures above $10^{-7}$ Torr, the RGA calibration is taken to be correct, so that

$$
\frac{\rho_{2}}{P_{2}}=\frac{1}{\alpha_{22}}=\frac{1}{k T}=3.3 \times 10^{16} \frac{\text { atoms } / \mathrm{cm}^{3}}{\text { Torr }},
$$

as required by the ideal gas law at room temperature.

Although $\alpha_{11}$ and $\alpha_{12}$ are not needed to find $\rho_{1}$, they can be measured. When the RGA was new, $\alpha_{12} / \alpha_{22}$, which is the ratio of $P_{1}$ and $P_{2}$ when $\rho_{1}=0$, 
was close to the manufacturer's estimate of $1 \%$. This measurement is easily done by admitting $\mathrm{H}_{2}$ until $P_{1}$ reaches 100 times its original value. When the RGA returned from being rebuilt, $\alpha_{12} / \alpha_{22}$ had become $9 \%$. The coefficient $\alpha_{11}$ can be found from $\mathrm{Eq} .75$ as follows:

$$
\begin{gathered}
\Delta P_{1}=\left(\alpha_{12}-2 \alpha_{11}\right) \Delta \rho_{2}, \\
\Delta P_{2}=\left(\alpha_{22}-2 \alpha_{21}\right) \Delta \rho_{2} . \\
\frac{\Delta P_{1}}{\Delta P_{2}}=\frac{\alpha_{12}-2 \alpha_{11}}{\alpha_{22}-2 \alpha_{21}}=\frac{\alpha_{12}-2 \alpha_{11}}{\alpha_{22}\left(1-2 \alpha_{21} / \alpha_{22}\right)} . \\
\frac{\alpha_{11}}{\alpha_{22}}=\frac{-\Delta P_{1}}{2 \Delta P_{2}}\left(1-2 \frac{\alpha_{21}}{\alpha_{22}}\right)+\frac{1}{2} \frac{\alpha_{12}}{\alpha_{22}} \approx \frac{-\Delta P_{1}}{2 \Delta P_{2}}+0.04 .
\end{gathered}
$$

In the approximation $\alpha_{21}=0$, this can be reduced to the more intuitive form

$$
\frac{\alpha_{11}}{\alpha_{22}}=\frac{P_{1}^{\text {on }}-\frac{\alpha_{12}}{\alpha_{22}} P_{2}^{\text {on }}}{2\left(P_{2}^{\text {off }}-P_{2}^{\text {on }}\right)}
$$

by substituting

$$
\begin{aligned}
& \Delta P_{1}=P_{1}^{\text {on }}-P_{1}^{\text {off }}, \\
& \Delta P_{2}=P_{2}^{\text {on }}-P_{2}^{\text {off }}, \\
& P_{1}^{\text {off }}=\frac{\alpha_{12}}{\alpha_{22}} P_{2}^{\text {off }} .
\end{aligned}
$$

In one test (23Jan90), $P_{2}$ decreased from 18 to $12 \times 10^{-9} \mathrm{mbar}$, so the true pressure of $\mathrm{H}$ was $12 \times 10^{-9} \mathrm{mbar}$. $P_{1}^{\mathrm{on}}$ was $2.8 \times 10^{-9} \mathrm{mbar}$, of which $1.1 \times$ $10^{-9}$ mbar was due to $\mathrm{H}_{2}$. Then $\alpha_{11} / \alpha_{22}=1.7 / 12=1 / 7$. The largest difference between the diagonal atomic $\left(\alpha_{11}\right)$ and molecular $\left(\alpha_{22}\right)$ hydrogen sensitivities comes from the quadrupole transmission factor. The difference in ionization cross section is $45 \%$ (see Table 8 ), whereas the total sensitivities were found to differ by a factor of 7 . Therefore, the quadrupole transmissivity for $\mathrm{H}_{2}$ must be five times that for $\mathrm{H}$. 
Table 8. Electron ionization cross sections at $60 \mathrm{eV}$.

\begin{tabular}{lc}
\hline \hline \multicolumn{1}{c}{ Process } & Cross section $\left(\mathrm{cm}^{2}\right)$ \\
\hline $\mathrm{e}+\mathrm{H} \rightarrow \mathrm{H}^{+}$ & $6.7 \times 10^{-17}$ \\
$\mathrm{e}+\mathrm{H}_{2} \rightarrow \mathrm{H}_{2}^{+}$ & $9.7 \times 10^{-17}$ \\
$\mathrm{e}+\mathrm{H}_{2} \rightarrow \mathrm{H}^{+}$ & $0.4 \times 10^{-17}$ \\
\hline
\end{tabular}

\section{Calculations of flow rates and recombination rate}

This section demonstrates that pressures measured by the RGA during steady discisarge in the $\mathrm{H}$ source on $12 \mathrm{~J}$ un90 agree with calculations of flow rates into and out of the lower chamber. The calculated flow rate out of the chamber includes a model of recombination on the walls. The level of agreement achieved with reasonable assumptions about the sizes of the model parameters supports the determination of $\mathrm{H}$ densities in the interaction region from the RGA readings.

The rate of flow into the chamber may be derived by adjusting the model used by Chan et al. to describe a similar $\mathrm{H}$ source. They calculated an atomic density $\rho_{c}=\rho_{\text {Chan }}$ just inside the end of their capillary when the supply pressure $P_{s}$ had the value $P_{\text {Chan }}$. For values of $P_{s}$ in the same range, $\rho_{c}$ should be

$$
\rho_{c}=\left(P_{s} / P_{\text {Chan }}\right) \rho_{\text {Chan }}
$$

The flow rate of these atoms, at a velocity $v$ determined by the temperature of the water-cooled wall, through a capillary of radius $r_{c}$, is

$$
Q_{c}=\rho_{c} v \pi r_{c}^{2}
$$

The skimmer aperture, also of radius $r_{c}$, a distance $D$, away permits a fraction $\left(r_{c} / 2 D_{s}\right)^{2}$ to pass into the lower chamber, so the final flow rate is

$$
Q_{\text {in }}=\rho_{c} v \pi\left(r_{c}^{2} / 2 D_{s}\right)^{2} .
$$


Table 9. Flow rate into the lower chamber on 12Jun90.

\begin{tabular}{cl}
\hline \hline Quantity & Value \\
\hline${ }^{\rho}$ Chan & $1.0 \times 10^{16} \mathrm{~cm}^{3}$ \\
$P_{\text {Chan }}$ & 0.40 Torr \\
$P_{s}$ & 0.045 Torr \\
$\rho_{c}$ & $1.1 \times 10^{15} \mathrm{~cm}^{3}$ \\
$r_{c}$ & $0.05 \mathrm{~cm}$ \\
$D_{s}$ & $2.9 \mathrm{~cm}$ \\
$v$ & $2.6 \times 10^{5} \mathrm{~cm} / \mathrm{sec}$ \\
$Q_{\text {in }}$ & $1.7 \times 10^{14} \mathrm{atoms} / \mathrm{sec}$ \\
& $=5.2 \times 10^{-6}$ Torr- $1 / \mathrm{sec}$ \\
\hline \hline
\end{tabular}

Evaluations of these quantities from measurements on 12Jun90 appear in Table 9.

The rate at which atoms leave the chamber is approximately twice $S_{2}$, the pumping speed for $\mathrm{H}_{2}$, since each molecule consists of two atoms. At the side of the cube, $S_{2}$ was $650 \mathrm{l} / \mathrm{sec}$, so atoms were being removed at a rate of $1300 \mathrm{l} / \mathrm{sec}$, where the liters referred to are prior to recombination. Here we have assumed that all of the $\mathrm{H}_{2}$ being pumped comes from recombination of $\mathrm{H}$, and that the recombination occurs in the cube. (Atoms that recombine in the drift tube will be pumped more slowly, but those that recombine in the tube to the pump will be pumped more quickly, so the errors in the latter assumption tend to cancel.) Since the RGA reading for $\mathrm{H}_{2}$ was $3.8 \times 10^{-9}$ Torr, the flow out of the chamber (in terms of Torr-l of $\mathrm{H}$ before recombination) is

$$
Q_{\text {out }}=4.9 \times 10^{-6} \text { Torr- } 1 / \mathrm{sec} \text {. }
$$

The recombination model derived in section 3.5.3 of Chapter 3 depends on $F_{c}$, the fraction of the wall covered by $\mathrm{H}$ atoms; $R_{c}$, the rate at which each atom collides with the wall; and $P_{r}$, the probability of recombination when two atoms meet at the wall. The equilibrium recombination rate $R_{e}$, which must be equal 
to the rates $Q_{\mathrm{in}}$ and $Q_{\mathrm{out}}$, thus determines the equilibrium number of atoms in the chamber

$$
N_{e}=\frac{R_{e}}{R_{c} P_{r} F_{c}}
$$

Then the pressure due to atoms is given by the ideal gas formula,

$$
P_{1}=\frac{N_{e} k T}{V}=\frac{R_{e} k T}{R_{c} P_{r} F_{c} V}
$$

where $V$ is the volume of the chamber. This can be made to agree with the value of $P_{1}$ obtained as described in section 1 by choosing plausible values of the model parameters as shown in Table 10. Thus there is no reason to doubt the $H$ densities calculated from the $R G A$ readings.

Table 10. Comparisons of calculated pressures including a model for recombination on the walls of the lower chamber.

\begin{tabular}{cl}
\hline \hline Quantity & Value \\
\hline$R_{c}$ & $10000 / \mathrm{sec}$ \\
$P_{r}$ & 0.7 \\
$F_{c}$ & 0.01 \\
$V$ & 201 \\
$P_{1}$ from $\mathrm{RGA}$ & $3.8 \times 10^{-9}$ Torr \\
$P_{1}$ when $R_{e}=Q_{\text {in }}$ & $3.7 \times 10^{-9}$ Torr \\
$P_{1}$ when $R_{e}=Q_{\text {out }}$ & $3.5 \times 10^{-9}$ Torr \\
\hline
\end{tabular}




\section{Appendix C. Measurement of focal volume characteristics}

The size and shape of the focal volume determine two important parameters of the experiment. One is the number of atoms irradiated at given gas density, that is, the size of electron signal to be expected from complete ionization of the gas. The other is the distribution of irradiance around the geometric focal point, which determines what portion of the total ionizations occur in the highirradiance core of the focal volume. A set of irradiance contours in three dimensions is therefore essential for any comparison of measured signals to theoretical predictions. I measured the irradiance distribution by moving the focal point in three dimensions, recording the fraction of the incident energy that passed through a pinhole, then fitting the data with a model that is appropriate for beams that are not diffraction limited. This process provided two independent estimates of the waist radius (the radius in the plane of best focus) that were in reasonable agreement. One estimate involved averaging many measurements at each position of the focal point, while the other used the highest single measurement from each set. Under optimal conditions, the waist radius was eight times that of a diffraction limited system diffraction limit, but at other times it was as large as ten times the diffraction limit.

\section{Experimental setup}

I measured the irradiance distribution under conditions as close as possible to those of the experiment. To avoid disturbing the alignment of the $f / 20$ focusing mirror, I left it in place and used the motor translators in the mirror assembly to move th's spot relative to the pinhole, which was placed near the center of the cube. Further, the usual chain of beamsplitters brought the laser beam to the focusing mirror. However, more attenuation was required to prevent burning 
new holes in the foil around the pinhole, so I placed a high reflectance dielectric mirror ahead of the beamsplitters and used the light that leaked through it. I selected a mirror with both front and back surfaces specified as $\lambda / 10$ for red light so that it would not distort the beam appreciably. The mirror was several meters ahead of the first beamsplitter so that the reflection was kept away from the UV detectors.

A pair of Hamamatsu model R1193U-02 vacuum photodiodes measured the incident and transmitted power levels. One photodiode sampled the incident power by taking the portion that passed through the last beamsplitter. At this position, the beam overfilled the photocathode. The other photodiode was at the point where the beam dump tube normally was attached to the cube. It was close enough to the focus that the diverging beam underfilled its photocathode. Thus neither measurement was sensitive to small misalignments of the photodiodes or to the motion of the focusing mirror. The photodiode signals were recorded on film by an oscilloscope. Switching the detectors and moving the cables verified that the photodiodes, cables, and oscilloscope amplifiers were interchangeable.

\section{Procedure}

I first centered the photodiodes with respect to the laser beam, then positioned the focus beyond the edge of the foil containing the pinhole and compared the photodiode readings. This established the ratio of signals that represented $100 \%$ transmission. As a byproduct, it also tested the uniformity of the irradiance across the laser beam. If the beam were perfectly uniform, the signal from the overfilled photodiode would be smaller than the signal from the underfilled photodiode by the ratio of the area of the photocathode to that of the incident beam. The diameter of the incident beam was set at 1.20 in. $(30.5 \mathrm{~mm})$ by an iris, while the photocathode diameter was $20 \mathrm{~mm}$, so the expected ratio of signals was $(20 / 30.5)^{2}=43 \%$. The actual ratio was $41 \%$, indicating that the irradiance of the beam did not decrease sharply near the edge. Comparing the photodiode 
readings also tested whether the photodiodes were responding to stray refle:tions of the beam as well as to the portion that came through the system of attenuators. Reflections were more significant in these measurements than in normal operation because the attenuation had been increased by a factor of more than 100. It was necessary to orient the photodiodes perpendicular to the path of the main beam and install several baffles to raise the correlation statistic $R^{2}$ between the two signals to $90 \%$.

After determining the ratio of signals corresponding to $100 \%$ transmission, I placed the spot on the pinhole by translating the focusing mirror while watching the photodiode signals on the oscilloscope. This process was complicated by the motion of the spot. Besides the shot to shot jitter, later estimated to average one hole radius $(12.5 \mu \mathrm{m})$, the average position of the spot frequently changed by several spot diameters in a matter of seconds. Once the pinhole was located, I scanned the spot across it, photographing the photodiode signals at each position along the scan, and taking both horizontal and vertical scans to check that the spot was circular. I also recorded one axial scan by aligning the spot to the pinhole for each axial position of the focusing mirror, then photographing the signals immediately. This set of data formed a smoother curve than any other, since the spot had less time to move before the measurement.

\section{The $M^{2}$ model}

I transformed my measurements of transmitted power into a representation of the irradiance distribution in the focal region by fitting the data to an $M^{2}$ model. This model (Marshall 1971, Sasnett 1989), which describes the propagation of a multimode laser beam, provides a good representation of many actual laser beams regardless of the details of their mode structure. The basic idea is that a multimode beam expands beyond its waist region more slowly than a diffraction limited single-mode $\left(\mathrm{TEM}_{00}\right)$ beam, which would have a smaller waist radius, but more quickly than a single-mode beam brought to the observed waist 
radius by different optics. It expands, in fact, at the same rate as a single-mode beam of intermediate waist radius, called the "embedded Gaussian". Thus only one parameter need be added to the usual single-mode formula to describe the multimode beam.

Specifically, the new parameter $M$ is defined by the relation

$$
M^{2}=W_{0} / w_{d}
$$

where $W_{0}$ is the radius of the actual waist, determined by the $1 / \mathrm{e}^{2}$ irradiance contour, and $w_{d}$ is the radius of a diffraction limited waist in the same optical system. If there were another single-mode beam, the embedded Gaussian, of waist radius $w_{0}=W_{0} / M$, it would expand with the axial coordinate $z$ according to

$$
w(z)=w_{0} \sqrt{1+\left(\frac{\lambda z}{\pi w_{0}^{2}}\right)^{2}} .
$$

The $M^{2}$ model states that the radius of the actual beam is larger than that of the embedded Gaussian by the same factor everywhere, hence

$$
W(z)=W_{0} \sqrt{1+\left(\frac{M^{2} \lambda z}{\pi W_{0}^{2}}\right)^{2}} .
$$

The distance over which $W(z)$ increases by $\sqrt{2}$ is then a modified Rayleigh length

$$
Z_{R}=\frac{\pi W_{0}^{2}}{M^{2} \lambda}
$$

The model may thus be fit to an actual multimode laser beam with the two parameters $W_{0}$ and $Z_{R}$, but with the extra constraint that the measured waist radius is $M^{2}$ times diffraction limited. 


\section{Analysis methods}

I used two basic methods of dealing with noise in the data due to shot-toshot motion of the focal spot in the plane of the pinhole. One is the familiar method of setting the mirror position, then taking the average of a series of readings. This removes random noise, but it cannot help much when the average position of the focal spot shifts during the measurement. The other method is based on the idea that only a single measurement is necessary if one knows enough about that measurement. If the center of the spot and the center of the pinhole coincide, and if the radial irradiance distribution is known, a single value of the power transmitted through the hole determines the spot size.

These conditions on the single-shot measurement are not actually very restrictive. When the spot moves randomly in the neighborhood of the pinhole, it must sometimes land very close to the center of the pinhole. The highest measured transmission from a large number of such samples can therefore be taken to represent a direct hit, provided it is not too different from the second and third highest. Of course, a reading far above all the others must be suspected of being caused by electrical noise or some other atypical condition. The second condition, knowledge of the radial distribution, is weak because only the central portion of the spot is sampled. For example, a perfect plane wave reduced to a finite diameter would focus to an Airy disk. A measurement extending from the center only as far as the half-power point, however, could only with difficulty distinguish the pattern from a Gaussian. If the first zero of the Airy distribution is at radius $R_{A}$, a Gaussian of waist radius $W_{0}=0.71 R_{A}$ is usually an acceptable substitute. In this representation, the fractional transmission $F(0)$ through a hole of radius $R$ perfectly centered on the focal spot is

$$
F(0)=\int_{0}^{R} r e^{-2 r^{2} / W_{0}^{2}} d r=1-e^{-2 R^{2} / W_{0}^{2}}
$$


where the manufacturer specified $R=25 \pm 2 \mu \mathrm{m}$ for the pinhole used in this case.

The single-shot technique can be extended somewhat to analyze data from scans across the pinhole. When the centers of the spot and the hole are a distance $r_{c}$ apart, the fractional transmittance $F\left(r_{c}\right)$ is less than $F(0)$. The $F\left(r_{c}\right)$ curve is Gaussian with width $\rho=\sqrt{R^{2}+r_{c}^{2}}$, so that

$$
F\left(r_{c}\right)=F(0) e^{-2 r_{c}^{2} / \rho^{2}}
$$

This formula can be used to fit the highest reading $F_{n}(p)$ in the set of $n$ readings at each position $p$ along the scan. When the spot is moving randomly, however, $r_{c}$ must derived from $p$. In doing this, one must remember that by selecting the highest of each set of readings, one converts the random spot displacement into a systematic displacement in the direction of the point $p_{0}$ of greatest overlap. Thus, if $J$ is the maximum radius of the jittering motion,

$$
r_{c}=\left|p-p_{0}\right|-J
$$

or it is zero if this expression is negative. I chose to plot the measured data with position $p$ as the axis. The model curves, therefore, appear wider than they would appear if plotted against $r_{c}$. Essentially, each half of the model curve is moved away from the center by a distance $J$, and the gap is filled by the constant value $F(0)$.

When analyzing the average values of the data sets, I could not use the method that is normally used when the spot jitters by small fractions of the hole diameter. That method is to average the readings at each value of $p$, fit the curve, and deconvolve using the shape of the pinhole. Instead, I constructed a model which averaged the fractional transmission $F\left(r_{c}\right)$ over a number of random spot positions $r_{c}$, weighing each by the probability of finding the spot at that position. The model also allowed the scan to be offset from the center of the hole by a 
specified distance. The random motion was specified by the standard deviation of the jitter distance, with all directions of motion being equally likely. Because the actual spot changed its average position from time to time, and because the random motion was probably greater in some directions than in others, the model achieved a loose fit to the data at best. In general, these fits require larger offsets than the single-point results suggest. They serve chiefly to test whether the average spot radius during a series of measurements was considerably larger than the smallest radius encountered in that series.

\section{Results}

\subsection{Waist radius from single-shot measurements}

The best indication of the waist radius under optimal conditions came from a series of three vertical scans in the plane of best focus on $20 \mathrm{Apr} 90$. These data are more consistent than the other data sets, apparently because the size of the waist was uniform from shot to shot and the jitter distance was unusually small. Figure 18 presents the maximum transmission values $F_{n}(p)$ from these scans with models corresponding to $W_{0}=32.7,34.7$, and $36.7 \mu \mathrm{m}, J=2 \mu \mathrm{m}$, and $p_{0}=-175 \mu \mathrm{m}$. The best fit (lowest $\chi^{2}$ ) occurs when $W_{0}=34.7 \mu \mathrm{m}$. This choice is supported by values of $F_{n}\left(p_{0}\right)$ from these scans and three more scans performed the same day. Five of these six estimates of $F(0)$ corresponded to spot radii between 34.4 and $35.4 \mu \mathrm{m}$. The sixth estimate was $45.2 \mu \mathrm{m}$, but the shape of the curve indicated that the spot really was unusually large during that scan.

I found a confidence interval for $W_{0}$ from the data of Fig. 18 by calculating values of $\chi^{2}$ per degree of freedom $\left(\chi^{2} / \mathrm{df}\right)$. Since there were 14 data points and three model parameters, the number of degrees of freedom (df) was fixed at 11 , but the variance of the data $\left(\sigma^{2}\right)$ had to be estimated. I first examined the four pairs of points obtained at equal positions along the scan. The higher of each pair was greater than the average of the pair by 1.1 to $3.8 \%$ of the average value, 


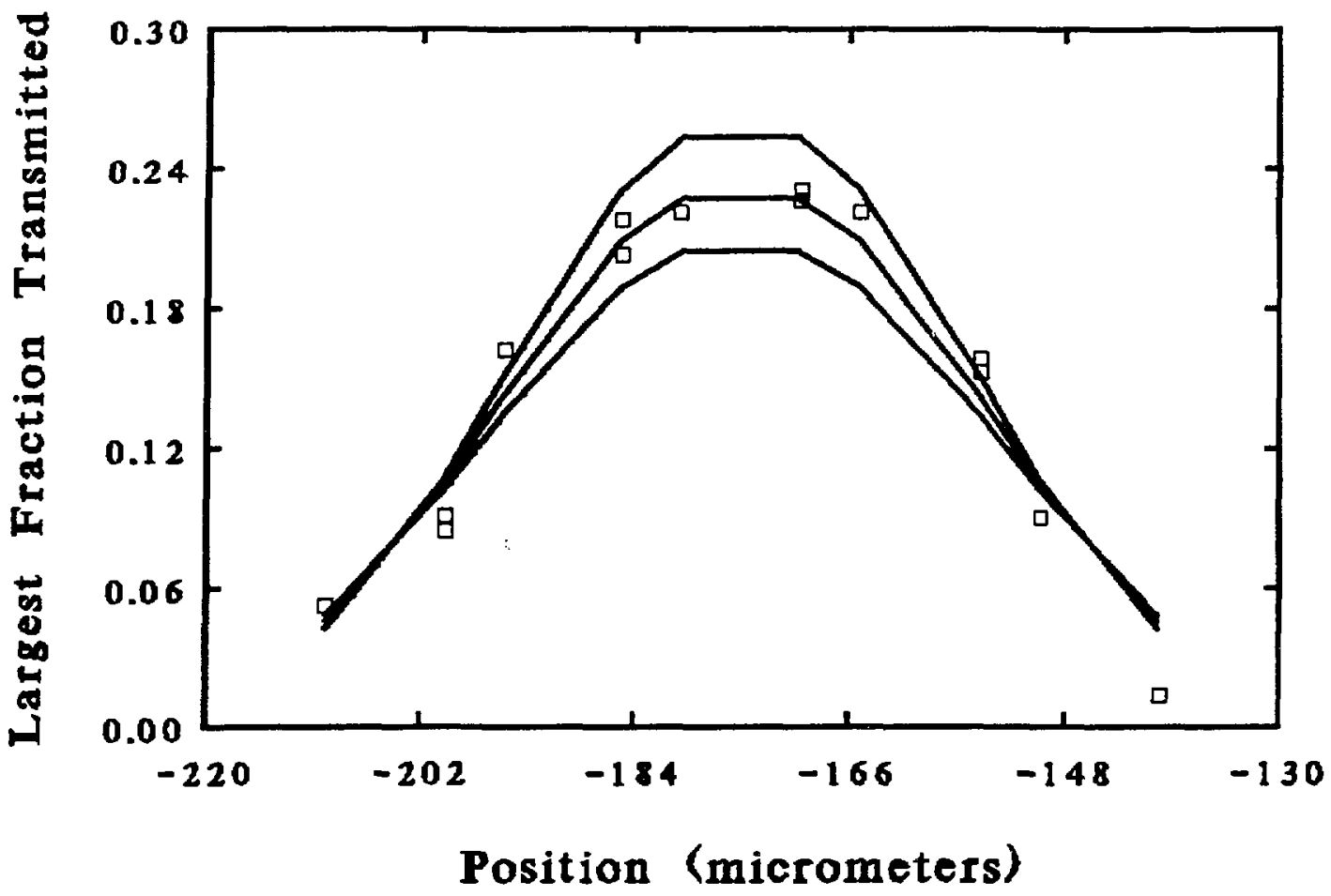

Figure 18. Highest values of transmission from vertical scan through focus. Data of 20Apr90 compared to models with $W_{0}=32.7,34.7$, and $36.7 \mu \mathrm{m}, J=2 \mu \mathrm{m}$, and $p_{0}=\cdots 175 \mu \mathrm{m}$.

so I tried setting $\sigma$ to $4 \%$ of the measured value $F_{n}(p)$, considering it likely that the error was multiplicative. The value of $\chi^{2} / \mathrm{df}$ was then 37 at best, increasing to 39 and 44 for the other values of $W_{0}$. These seemed too high, suggesting that my first estimate of the variance was too small. I also noticed that $3 / 4$ of $\chi^{2}$ came from the single point at $p=-140 \mu \mathrm{m}$. I deleted that point on the assumption that the sample size was too small to guarantee a direct hit on the pinhole. I then estimated $\sigma$ from $\chi^{2} / \mathrm{df}$ itself, finding that $\chi^{2} / \mathrm{df}=1$ when $\sigma=.118 F_{n}(p)$. This is a reasonable value, considering how the data were collected. Suppose the highest reading $F_{n}(p)$ in a data set is actually equal to the largest possible fractional transmission, but that it was missed when the sample was taken. The second 
highest value $F_{n-1}(p)$ would then be used in the calculations. The fractional error thus introduced is

$$
E_{n}(p)=\frac{F_{n}(p)-F_{n-1}(p)}{F_{n-1}(p)}
$$

Values of $E_{n}(p)$ derived in this way from the measurements of Fig. 18 range from $5 \%$ to $129 \%$, with a mean of $32 \%$. In these data sets, $n$ was 11,12 , or 13 . Actual errors should be somewhat less than $E_{n}(p)$, since the ideal reading is not always missed, so an estimate of $12 \%$ is quite plausible. With this estimate, $\chi^{2} / \mathrm{df}$ varied with $W_{0}$ as shown in Table 11. The distribution of $\chi^{2} / \mathrm{df}$ with ten degrees of freedom is such that $\chi^{2} / \mathrm{df}<1.6$ with $90 \%$ probability. Thus the confidence interval for $W_{0}$ is $\pm 2 \mu \mathrm{m}$.

Table 11. Chi square per degree of freedom from three scans in the plane of best focus. Best-fit waist radius $W_{0}=34.7 \mu \mathrm{m}$. Ten degrees of freedom.

\begin{tabular}{ccc}
\hline \hline$\Delta W(\mu \mathrm{m})$ & $\chi^{2} / \mathrm{df}\left(W_{0}-\Delta W\right)$ & $\chi^{2} / \mathrm{df}\left(W_{0}+\Delta W\right)$ \\
\hline 0 & 1.0 & \\
1 & 1.1 & 1.2 \\
2 & 1.8 & 2.0 \\
3 & 2.6 & 3.0 \\
4 & 3.3 & 3.8 \\
5 & 4.8 & 5.5 \\
\hline \hline
\end{tabular}

\section{2 $Z_{R}$ and $M^{2}$ from single-shot measurements}

The size of the beam at points other than the waist was best characterized by the series of single-shot measurements taken along the beam axis on 27Apr90. Some of these measurements were taken as an axial scan, while others were extracted from transverse scans across the spot in different planes. The measurements have been combined into a composite axial scan through focus (Fig. 19). Each measured point is the largest fractional transmission from a set 


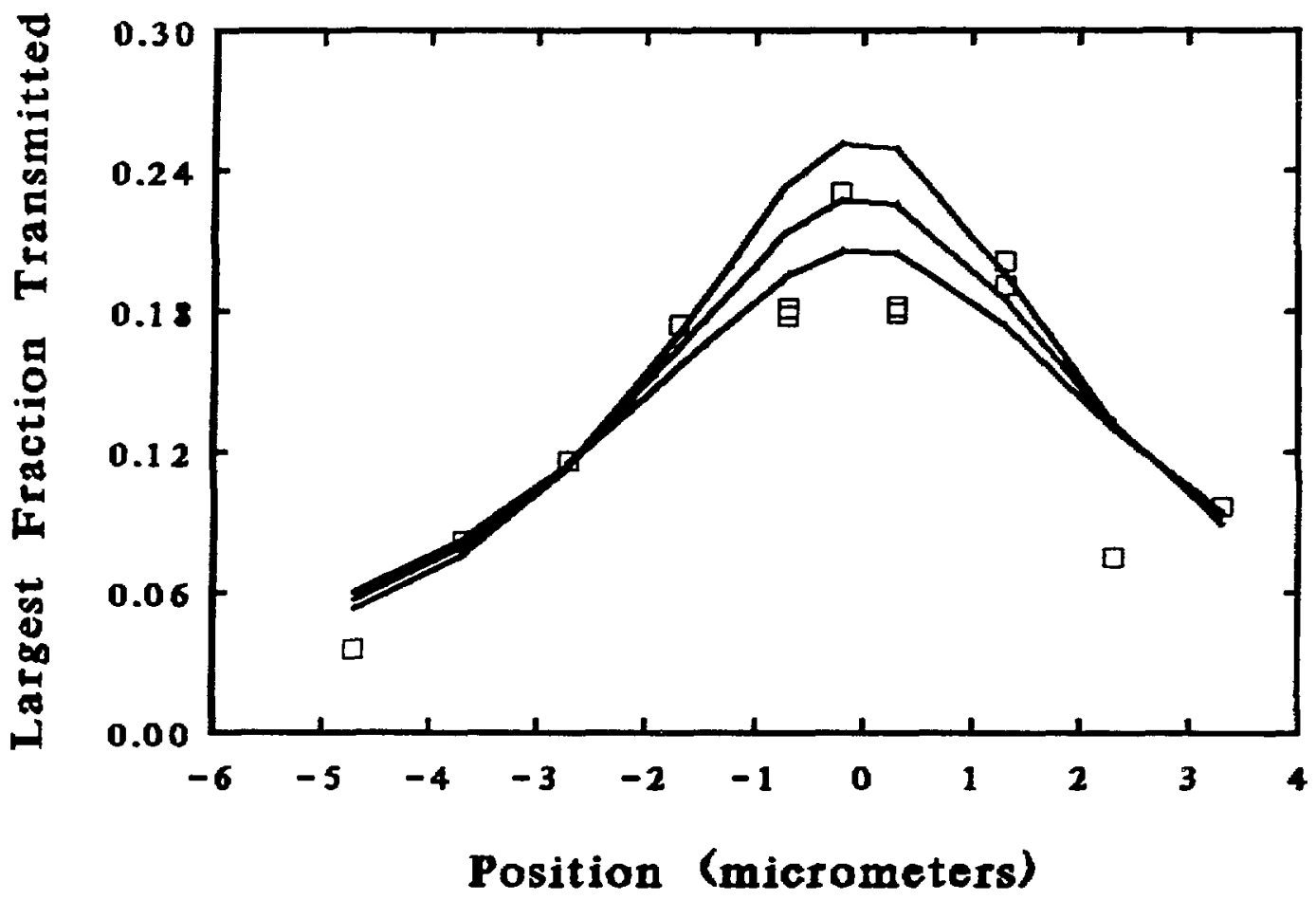

Figure 19. Highest values of transmission from axial scan through focus. Scan is a composite of points collected as an axial scan and points selected from transverse scans in different planes. Data of $27 \mathrm{Apr} 90$ are compared to $M^{2}$ models with $W_{0}=32.7,34.7$, and $36.7 \mu \mathrm{m}$, reading from highest to lowest. $M^{2}=6$ in each case.

of readings, representing the quantity $F(0)$ at an axial position $z$. (There was actually one measurement of 0.34 in the same set as the highest point plotted, but it was rejected as being too different from the others, as mentioned in section 4. This value of $F(0)$ corresponds to a spot radius of $27.4 \mu \mathrm{m}$.) The figure also displays the $M^{2}$ model with three sets of the two model parameters. The middle curve uses $W_{0}=34.7 \mu \mathrm{m}$ and $M^{2}=6$, which provide the best fit. The other curves have values of $W_{0}$ larger and smaller by $2 \mu \mathrm{m}$, with the same value of $M^{2}$. 
I again computed $\chi^{2} / \mathrm{df}$ to derive a confidence interval for $M^{2}$. In this derivation, I took $W_{0}$ as a known quantity. The two parameters of the fit were then $M^{2}$ (or equivalently $Z_{R}$ ) and the axial position of best focus $z_{0}$. There were 13 data points, hence 11 degrees of freedom. With $\sigma$ estimated to be $11.8 \%$ of the measured value, as before, the minimum value of $\chi^{2} / \mathrm{df}$ was 3.1 at $z_{0}=3.3 \mathrm{~mm}$ and $Z_{R}=2.4 \mathrm{~mm}$. The low points at $z=-4.6,2.4$ contributed $2 / 3$ of $\chi^{2}$ in this case. When these points were removed from the calculation, leaving nine degrees of freedom, the minimum value of $\chi^{2} / \mathrm{df}$ was 1.05 at $z_{0}=3.2 \mathrm{~mm}$ and $Z_{R}=2.6 \mathrm{~mm}$. Complete results appear in Table 12. For $\mathrm{df}=9, \chi^{2} / \mathrm{df}<1.63$ with $90 \%$ confidence, so the bounds on $M^{2}$ are 5.1 and 6.5 , while the bounds on $Z_{R}$ are 2.4 and $3.1 \mathrm{~mm}$. Returning to the full data set, an estimate of $\sigma=20 \%$ of each reading produced the results in Table 13. The confidence intervals estimated from this table are only a little broader than the first set, being 5.2 to 7.4 for $M^{2}$ and 2.1 to $3.0 \mathrm{~mm}$ for $Z_{R}$. I calculated one more $\chi^{2} / \mathrm{df}$ table (Table 14) to see whether the confidence range of $M^{2}$ depended sensitively on the value assumed for $W_{0}$. This time, I assumed $z_{0}=3.3 \mathrm{~mm}$ and varied both $W_{0}$ and $M^{2}$. Only the width of the confidence range for $M^{2}$ was found to depend on $W_{0}$, though the corresponding values of $Z_{R}$ increase as $W_{0}^{2}$. A conservative overall estimate is therefore

$$
M^{2}=6.2 \pm 1, \quad Z_{R}=2.6 \pm 0.5 \mathrm{~mm}
$$

The value of $M^{2}$ derived from the axial scan can be checked against a transverse scan at a distance from the focal plane that is comparable to $Z_{R}$. Figure 20 presents two such scans taken on $27 \mathrm{Apr} 90$ at a distance of $3.3 \mathrm{~mm}(z=0 \mathrm{~mm})$ that agree reasonably well with the model curve. There is one extra set of measurements near the peak which proved that the spot had not shifted during the second scan. Notice that the scale of the plot is much larger than that of the scans in the focal plane. As expected, many points fall below the model, since 
Table 12. Chi square per degree of freedom for an axial scan with nine degrees of freedom (two data points omitted). $M^{2}$ and $Z_{R}$ are equivalent variables in this table and the next, since $W_{0}$ is fixed at $35 \mu \mathrm{m}$. Values within the $90 \%$ confidence range are emphasized.

\begin{tabular}{cc|ccccc}
\hline \hline$M^{2}$ & $Z_{R}$ & $z_{0}=3.1$ & 3.2 & 3.3 & 3.4 & 3.5 \\
\hline 8.62 & 1.8 & 15.35 & 13.90 & 13.08 & 12.87 & 13.28 \\
7.76 & 2.0 & 7.31 & 6.52 & 6.10 & 6.07 & 6.42 \\
7.05 & 2.2 & 3.48 & 3.05 & 2.86 & 2.91 & 3.21 \\
6.47 & 2.4 & 1.76 & 1.54 & 1.48 & 1.58 & 1.84 \\
5.97 & 2.6 & 1.14 & 1.05 & 1.06 & 1.18 & 1.40 \\
5.54 & 2.8 & 1.09 & 1.08 & 1.14 & 1.26 & 1.46 \\
5.17 & 3.0 & 1.34 & 1.38 & 1.46 & 1.58 & 1.75 \\
4.85 & 3.2 & 1.73 & 1.80 & 1.89 & 2.02 & 2.17 \\
4.56 & 3.4 & 2.19 & 2.27 & 2.37 & 2.49 & 2.63 \\
\hline \hline
\end{tabular}

Table 13. Chi square per degree of freedom for an axial scan with eleven degrees of freedom (all data points used).

\begin{tabular}{cc|ccccc}
\hline \hline$M^{2}$ & $Z_{R}$ & $z_{0}=3.1$ & 3.2 & 3.3 & 3.4 & 3.5 \\
\hline 8.62 & 1.8 & 4.60 & 4.11 & 3.82 & 3.72 & 3.81 \\
7.76 & 2.0 & 2.35 & 2.09 & 1.93 & 1.90 & 1.98 \\
7.05 & 2.2 & 1.42 & 1.27 & 1.20 & 1.20 & 1.27 \\
6.47 & 2.4 & 1.12 & 1.05 & 1.02 & 1.04 & 1.10 \\
5.97 & 2.6 & 1.13 & 1.10 & 1.10 & 1.13 & 1.19 \\
5.54 & 2.8 & 1.30 & 1.29 & 1.31 & 1.34 & 1.39 \\
5.17 & 3.0 & 1.53 & 1.54 & 1.56 & 1.60 & 1.65 \\
4.85 & 3.2 & 1.79 & 1.81 & 1.83 & 1.87 & 1.91 \\
4.56 & 3.4 & 2.04 & 2.07 & 2.10 & 2.13 & 2.17 \\
\hline \hline
\end{tabular}

these sets of 11 to 15 readings did not always include the highest possible fractional transmission. They were less likely to do so on 27 Apr90 than on 20 Apr90 because the jitter was larger, roughly $\pm 10 \mu \mathrm{m}$.

I compared the jitter radius $J$ from the model to estimates reached in a different way. This involved fairing a smooth curve through the average readings for different points in a transverse scan, taking the slope at each value of 
Table 14. Chi square per degree of freedom for an axial scan with eleven degrees of freedom, assuming $z_{0}=3.3 \mathrm{~mm}$.

\begin{tabular}{c|ccccc}
\hline \hline$M^{2}$ & $W_{0}=33$ & 34 & 35 & 36 & 37 \\
\hline 8.0 & 3.12 & 2.67 & 2.34 & 2.13 & 2.01 \\
7.5 & 2.11 & 1.80 & 1.59 & 1.47 & 1.43 \\
7.0 & 1.51 & 1.31 & 1.17 & 1.10 & 1.10 \\
6.5 & 1.27 & 1.12 & 1.02 & 0.98 & 0.99 \\
6.0 & 1.31 & 1.18 & 1.09 & 1.05 & 1.06 \\
5.5 & 1.57 & 1.43 & 1.33 & 1.27 & 1.26 \\
5.0 & 1.99 & 1.83 & 1.70 & 1.61 & 1.57 \\
4.5 & 2.53 & 2.33 & 2.16 & 2.03 & 1.94 \\
4.0 & 3.14 & 2.89 & 2.67 & 2.49 & 2.36 \\
\hline \hline
\end{tabular}

the scan coordinate $p$, and interpreting the fluctuations in the measurements as fluctuations in $p$ by dividing the size of the fluctuation by the slope of the faired curve. When this same process was applied to similar measurements using the red alignment beam and the alignment pinhole, the estimated jitter was $\pm 2 \mu \mathrm{m}$. This appeared to be the lowest value attainable with the focusing mirror and the pinhole rigidly attached to the table. When this method is applied to the three scans of Fig. 18 and another scan that day, it indicates values of $J$ between 2 and $15 \mu \mathrm{m}$, with an average value of $6 \mu \mathrm{m}$. This confirms that the values from the model are of the right order of magnitude.

\subsection{Average waist radius from averaged readings}

Comparisons of average transmissions to the random-motion model show that the average size of the beam waist was larger than that measured from the highest transmission in each set, and that the difference was greater on 27Apr90 than on 20Apr90. Figure 21 presents peak-transmission values from two scans in the plane of best focus on $27 \mathrm{Apr} 90$. These are in reasonable agreement with 


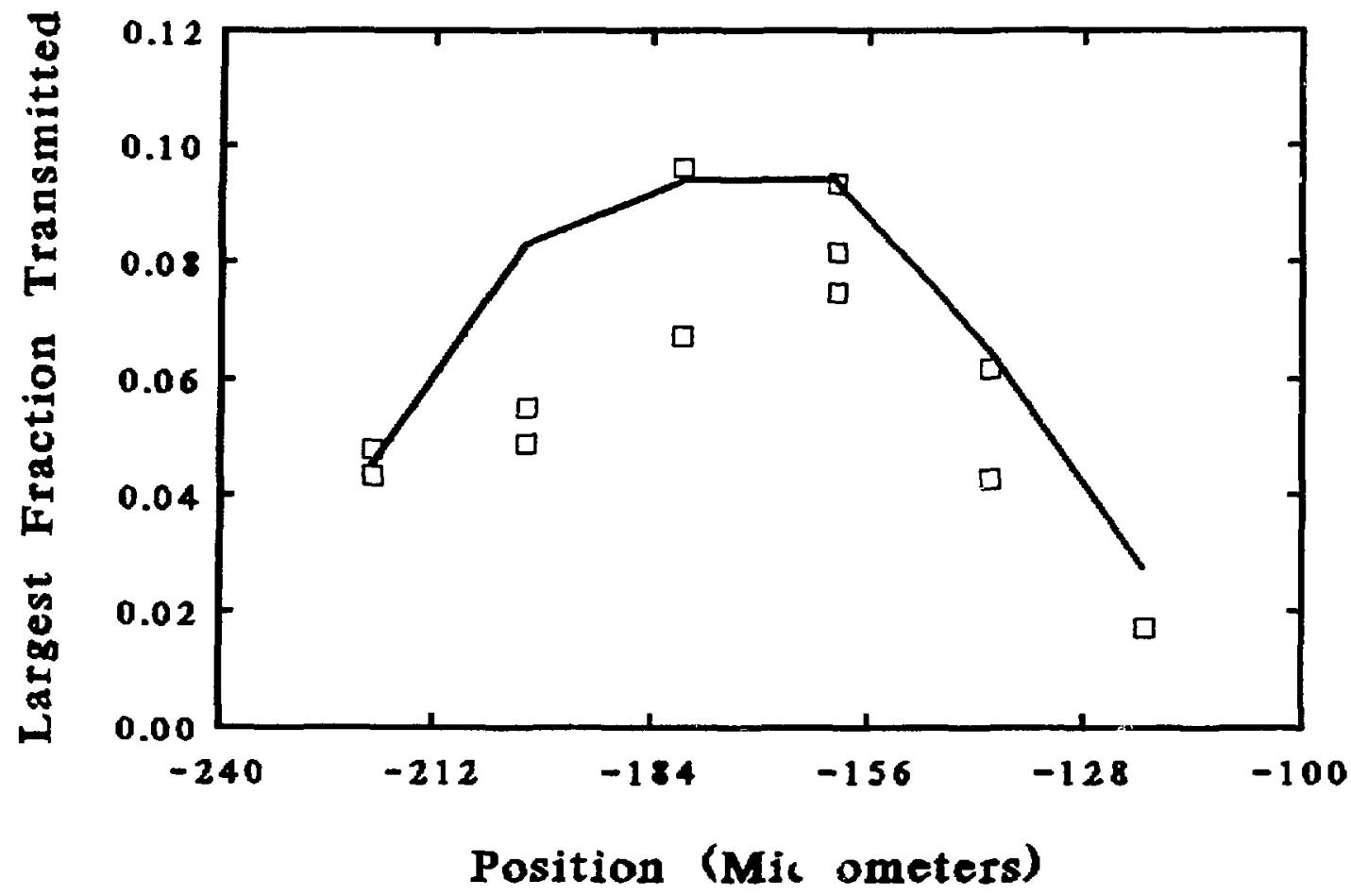

Figure 20. Highest values of transmission from transverse scan $3.3 \mathrm{~mm}$ from best focus. Data of $27 \mathrm{Apr} 90$ are compared to $M^{2}$ model with $W_{0}=35 \mu \mathrm{m}$ and $M^{2}=6$.

the model for $W_{0}=34.5 \mu \mathrm{m}$ and $J=10 \mu \mathrm{m}$. Figure 22 shows the averagetransmission values from the same scans. These clearly do not fit the model for any value of the offset parameter, but they do fit a model with $W_{0}=45 \mu \mathrm{m}$, as shown in Fig. 23. Averaged data from $20 \mathrm{Apr} 90$, on the other hand, agree with the $W_{0}=34.5 \mu \mathrm{m}$ model, as shown in Fig. 24. These are average transmissions from the same scans used in Fig. 18. The model, however, has been adjusted to a jitter of $J=15 \mu \mathrm{m}$ with offsets of 10,15 , and $20 \mu \mathrm{m}$. The assumed jitter distance in this case is much greater than that derived from single-shot analysis. The difference is probably due to the fact that the average values are sensitive to jitter in eny direction, while the peak values in these vertical scans are specifically 


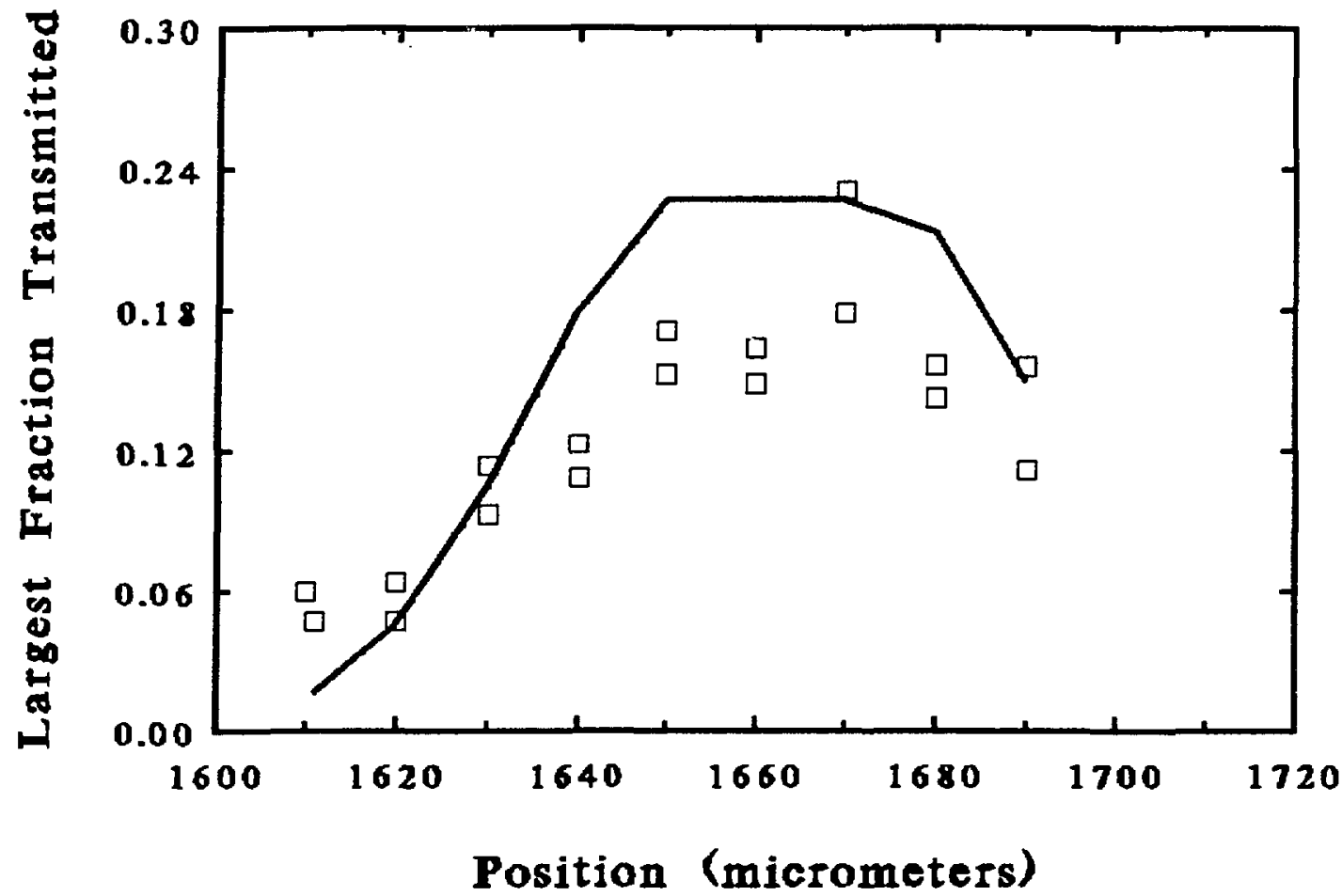

Figure 21. Highest values of transmission from two horizontal scans through focus. Data of $27 \mathrm{Apr} 90$ are compa: i to model with $W_{0}=$ $35 \mu \mathrm{m}$.

those with vertical displacements. The analysis then indicates that the horizontal jitter was stronger than the vertical jitter at that time.

\section{Discussion}

The result of the measurement is that the $M^{2}$ model with $W_{0}=35 \pm 2 \mu \mathrm{m}$ and $M^{2}=6.2 \pm 1$ is a useful representation of the laser beam near an $f / 20$ focus under optimal conditions. At other times, however, $W_{0}$ may be as large as $45 \mu \mathrm{m}$. The waist radius may be stable near one extreme or the other for considerable periods, or it may fluctuate shot to shot. Compared to a diffraction limited Airy pattern, the measured focus is 8 to 10 times diffraction limited. 


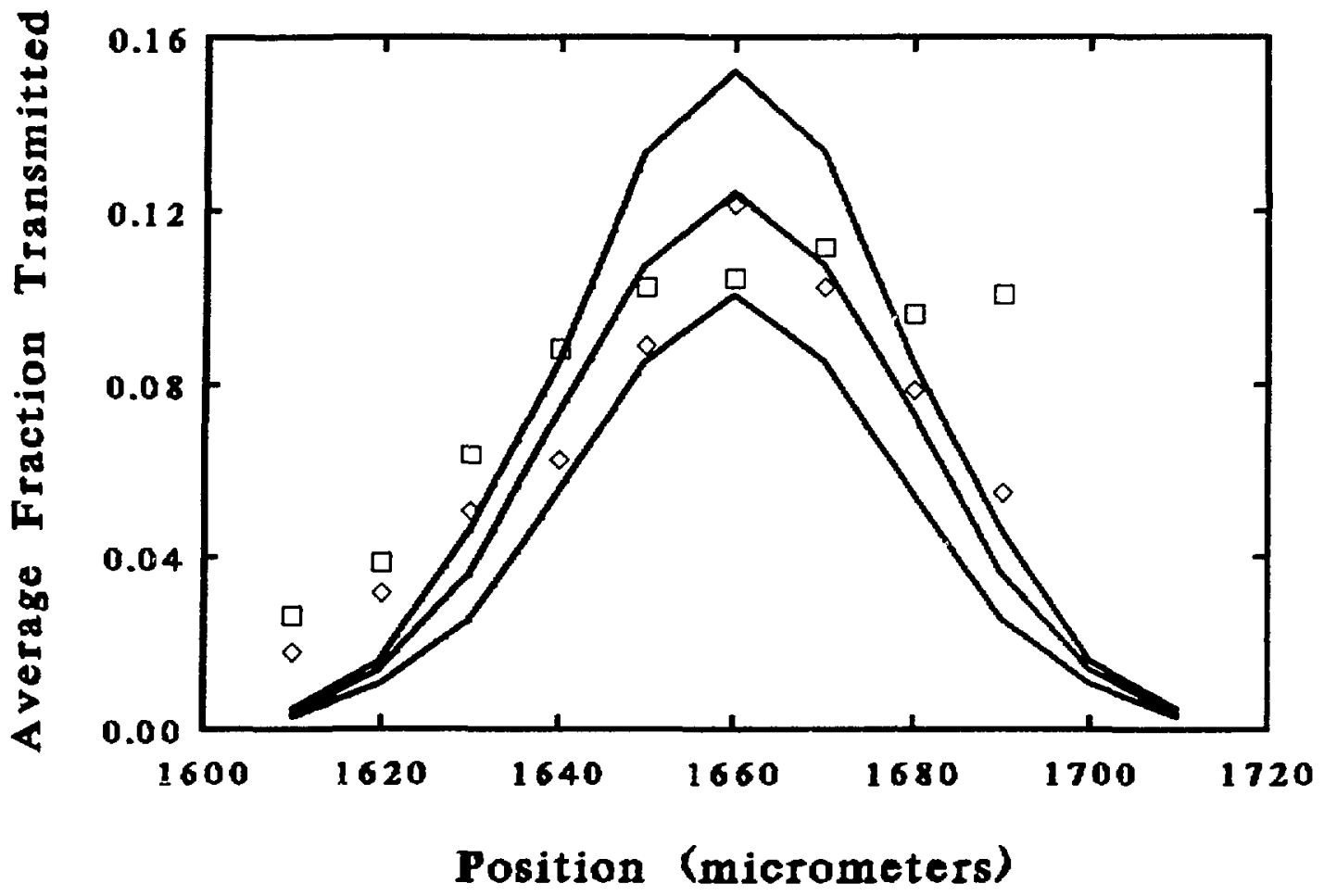

Figure 22. Average values of transmission from the same scans as in Fig. 21, compared to model with $W_{0}=35 \mu \mathrm{m}$.

The extra constraint in the $M^{2}$ model is satisfied reasonably well. In the case of $W_{0}=35 \mu \mathrm{m}$ and $M^{2}=6.2$, the diffraction limited radius $w_{d}$ is determined to be $5.6 \mu \mathrm{m}$, corresponding to an Airy first zero radius of $R_{A}=8.0 \mu \mathrm{m}$. The value from the Airy formula is $R_{A}=6.1 \mu \mathrm{m}$.

If the average spot expanded suddenly from 35 to $45 \mu \mathrm{m}$ during collection of electron spectra, the effect would be dramatic. Assuming no change in pulse energy or pulse length, the irradiance at focus changes as $W_{0}^{-2}$, and the rate of a third order nonlinear process changes as $W_{0}^{-6}$, while the number of atoms in the focal volume changes approximately as $W_{0}^{2}$ when the high-irradiance volume extends beyond the limits of the atomic beam. The size of the $S_{0}$ peak of atomic hydrogen would thus decrease by the factor $(35 / 45)^{4}=0.37$. Therefore, when 


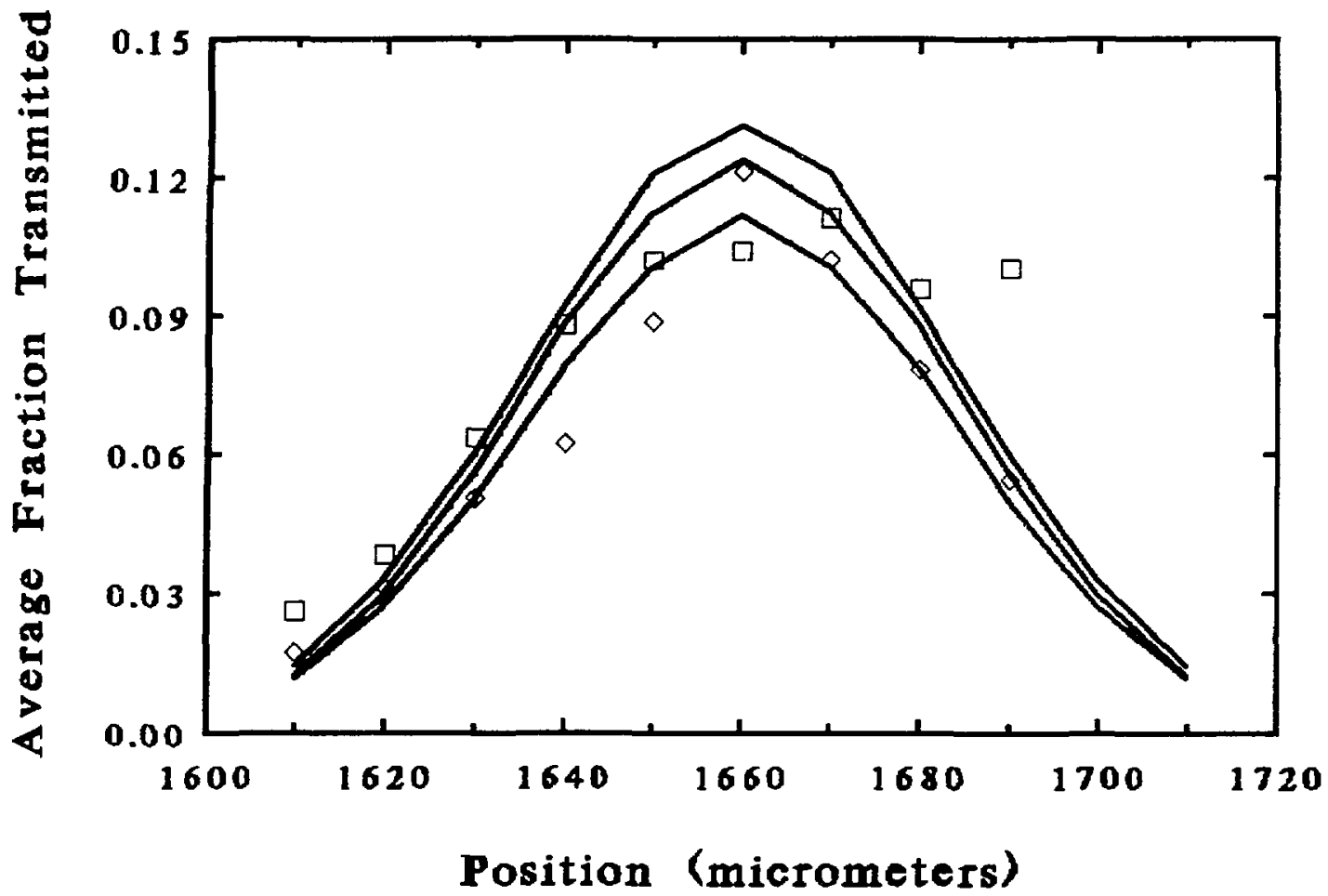

Figure 23. Average values of transmission from the same scans as in Fig. 21, compared to model with $W_{0}=45 \mu \mathrm{m}$.

two electron spectra corresponding to equal pulse energies differ by factors of three or four, it is reasonable to say that the higher electron counts are valid for the irradiance calculated with $W_{0}=35 \mu \mathrm{m}$, but the lower counts were very likely produced at lower irradiance. 


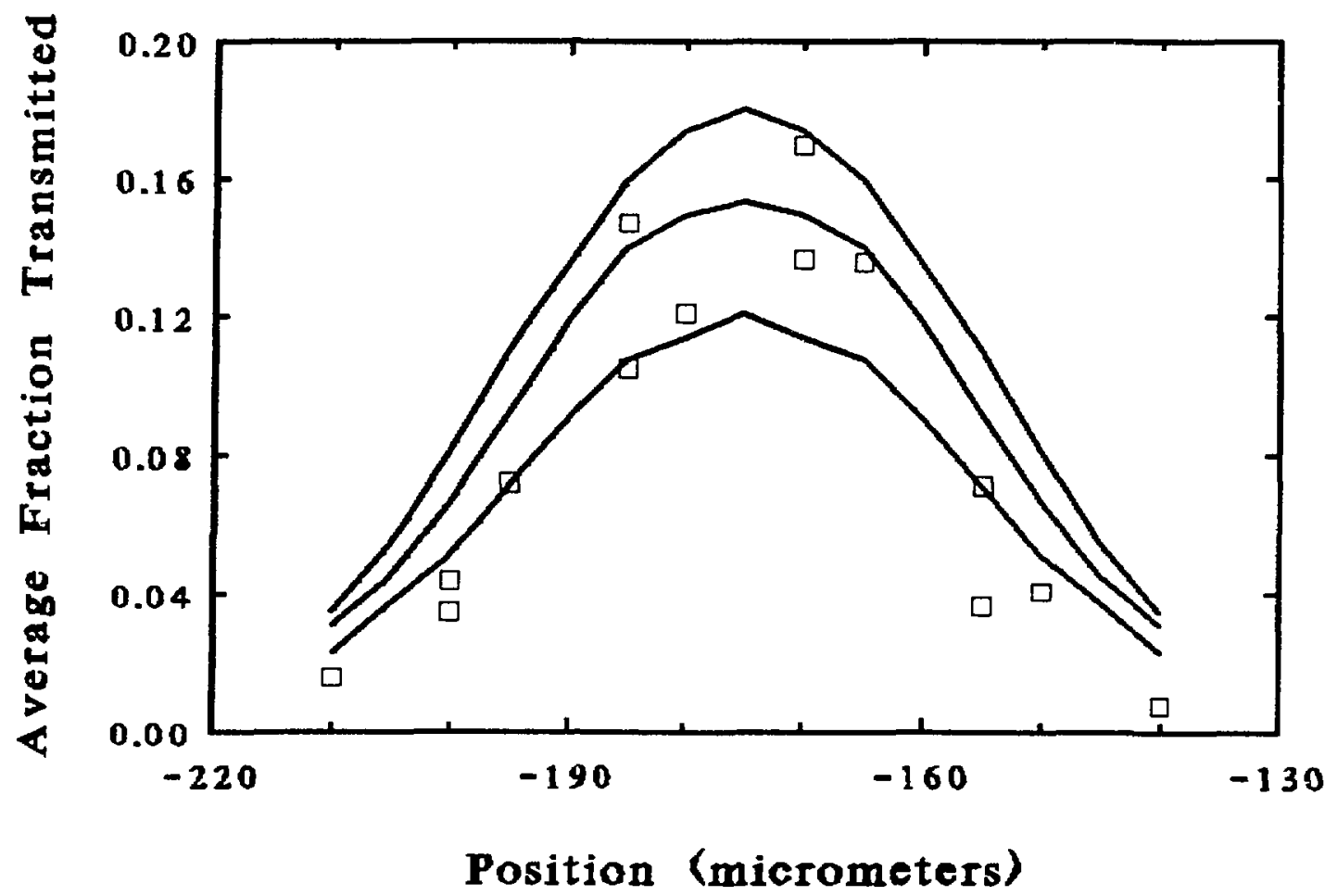

Figure 24. Average values of transmission from vertical scans of 20Apr90 compared to model with $W_{0}=35 \mu \mathrm{m}$. Values of offset are 10,15 , and $20 \mu \mathrm{m}$, reading highest to lowest. 


\section{Appendix D. Microchannel plate (MCP) electron detection system}

This appendix describes the electron detection system, consisting of an MCP assembly, a high-voltage power supply, a transient digitizer, and other equipment associated with them. The important parameter of the system is the gain $G_{M}$, which is the average signal recorded in the data file for each detected electron. I measured $G_{M}$ directly, and also measured the gain of the MCP assembly by itself to compare with available measurements of the response function of the transient digitizer by itself. The appendix concludes with a comparison between the measured MCP gain and the manufacturer's specifications, and three additional observations concerning the detection system.

\section{Description}

The electron spectra reported in this work were collected by an R. M. Jordan MCP detector. It contained two Galileo MCP-18B or equivalent microchannel plates in chevron configuration. Each plate was 24.7 to $24.8 \mathrm{~mm}$ in diameter and 0.41 to $0.46 \mathrm{~mm}$ thick. The channel diameter was $10 \mu \mathrm{m}$, and the channel spacing was $12.5 \mu \mathrm{m}$ maximum. The input aperture, located $13 \mathrm{~mm}$ from the first microchannel plate, was $19.0 \mathrm{~mm}$ in diameter. This aperture was part of the Faraday cage around the drift region. It supported a grid whose transmission fraction was $82 \%$. Materials used, besides the microchannel plates, were 304 stainless steel, 6061 aluminum, nickel, and alumina. The output impedance of the detector was $50 \Omega$, which matched the input impedance of the LeCroy TR8818 transient digitizer and the impedance of the cable between them. A $1 \mu \mathrm{f}$ capacitor in the output line blocked the positive dc voltage that was present on the anode. The entire detector assembly is usually referred to as "the MCP". 
A Power Designs model 1556B power supply provided positive high voltage for the MCP. A resistor chain divided the applied voltage into individual accelerating potentials as listed in Table 15. The output voltage of the power supply, measured by a digital multimeter with a high voltage probe, agreed with the setting of the controls within a few volts. The fine-adjustment control did not go to zero, so the voltages used were round numbers plus $9 \mathrm{~V}$.

Table 15. Potentials within MCP assembly for supply voltage of $2.209 \mathrm{kV}$.

\begin{tabular}{ccl}
\hline \hline $\begin{array}{c}\text { Resistor value } \\
(\mathrm{M} \Omega)\end{array}$ & $\begin{array}{c}\text { Volts across } \\
\text { resistor }\end{array}$ & $\begin{array}{c}\text { Where } \\
\text { applied }\end{array}$ \\
\hline 0.730 & 256 & ground to first plate \\
2.507 & 888 & across first plate \\
2.512 & 890 & across second plate \\
0.492 & 175 & second plate to anode \\
\hline \hline
\end{tabular}

\section{Measurement of system gain}

The system gain relates the signal recorded in a data file to the number of electrons that struck the MCP. The system consisted of the MCP, the TR8s18 transient digitizer, the CAMAC and GPIB electronics, the computer, and the cables connecting them. This section describes the process of measuring the gain of the total system, which is presented in Table 16 as a function of applied voltage. Section 3 describes measurements of the MCP and TR8818 gains separately.

The response of the entire system was measured by using the RGA filament as a source of single electrons. The measurement required a trigger signal to indicate when a pulse appeared in the MCP output, and a computer program to store the reading from the TR8818. The trigger came from a Tektronix 7104 oscilloscope connected in parallel with the input of the TR8818. The stop trigger for the TR8818 was produced just as in normal operation, but using the gate 
Table 16. Gain of the electron detection system consisting of MCP detector and transient digitizer.

\begin{tabular}{cc}
\hline \hline $\begin{array}{c}\text { Power supply } \\
\text { voltage }(\mathrm{kV})\end{array}$ & $\begin{array}{c}\text { Average signal (mV) } \\
\text { per detected electron }\end{array}$ \\
\hline 2.209 & $7.2 \pm 0.4$ \\
2.309 & $11.2 \pm 0.6$ \\
2.409 & $17.1 \pm 0.6$ \\
\hline \hline
\end{tabular}

output from the oscilloscope in place of the photodiode signal. The TR8818 reading of the pulse that triggered the oscilloscope therefore came at the same point in each data set. A modified version of the NEWRUN program located the signal and stored it. After storing 2400 readings, the program wrote a data file in the usual format, but representing single readings $v s$. trigger number rather than average signal vs. time. Two such files were stored for $2.209 \mathrm{kV}$ applied voltage, one for $2.309 \mathrm{kV}$, and two for $2.409 \mathrm{kV}$. Figure 25 presents histograms extracted from these files.

The number of interest was the average TR8818 output per detected electron at each applied voltage. This number was derived from each histogram by dividing its area by the number of detected electrons. The histograms in Fig. 25 are not complete, however. They do not include MCP output pulses that were too small to trigger the oscilloscope. Most of the missing pulses belong in the $0 \mathrm{mV}$ bin. The following estimate of the number of missing pulses provides an error estimate for each value of system gain.

The number of detected electrons was approximately equal to the number of triggers, but was somewhat higher. The oscilloscope's trigger level was set at $10 \mathrm{mV}$, just above the noise produced by the TR8818 at the end of a CAMAC cycle. Pulse heights ranged up to $200 \mathrm{mV}$ with $2.2 \mathrm{kV}$ applied to the resistor chain, and up to $450 \mathrm{mV}$ with $2.4 \mathrm{kV}$ applied. The number of pulses with 
Appendix D. Microchannel plate (MCP) electron detection system

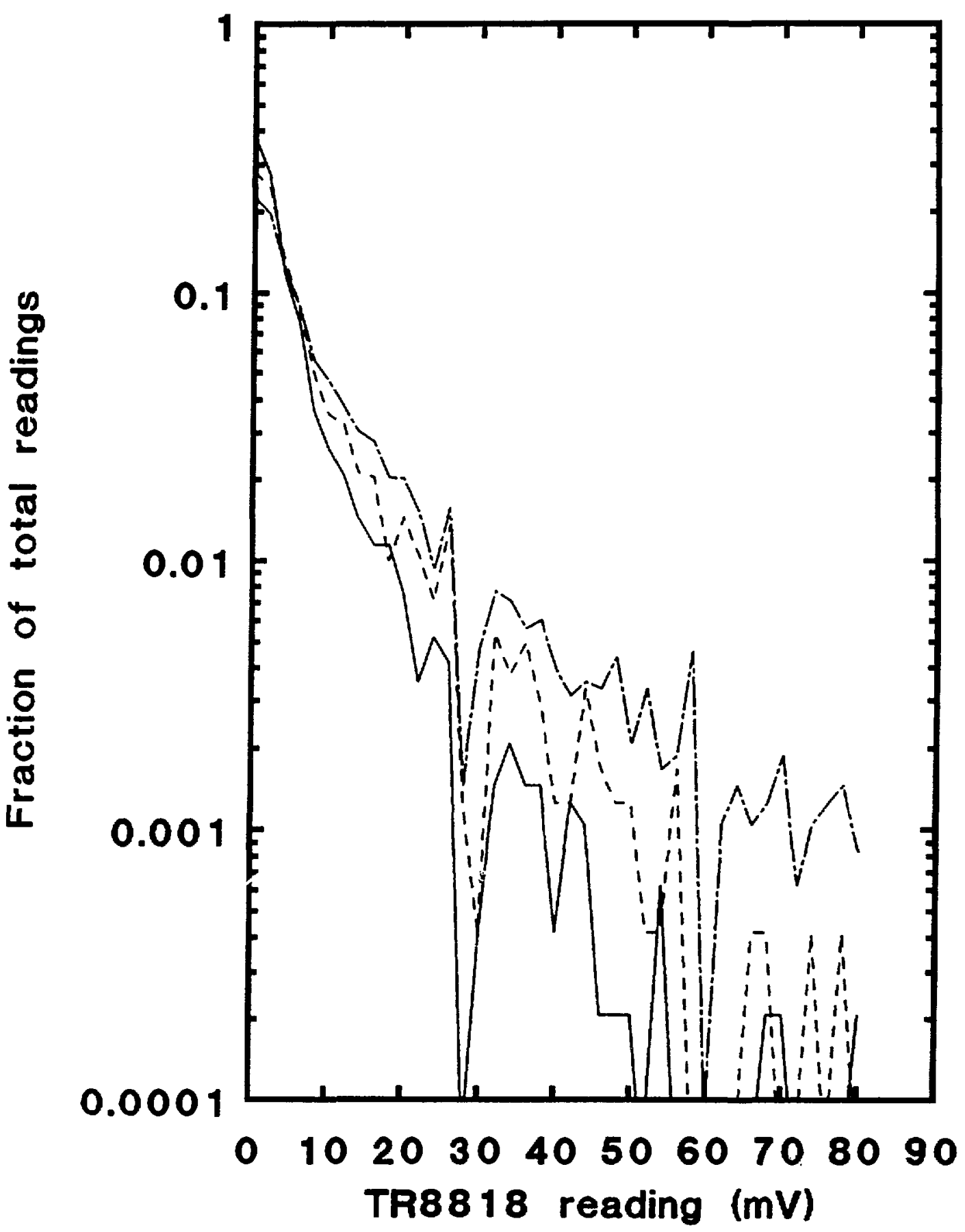

Figure 25. Distributions of recorded signals from single electrons detected by the MCP at three applied voltages. The solid curve represents 4800 readings at $2.2 \mathrm{kV}$ supply voltage. The dashed curve represents 2400 readings at $2.3 \mathrm{kV}$ supply voltage. The dot-dash curve represents 4800 readings at $2.4 \mathrm{kV}$ supply voltage. 
heights less than $10 \mathrm{mV}$ may be estimated by extending the curve of TR8818 readings backward to $0 \mathrm{mV}$ along an exponential fit to the points at $2-8 \mathrm{mV}$. Estimated totals of detected electrons from this procedure were 5327,2695 , and 5130 at $2.209,2.309$, and $2.409 \mathrm{kV}$ respectively. The upper and lower bounds in Table 16 were calculated from the numbers of triggers and the exponential estimates respectively.

One final adjustment must be applied to the signals described by the histograms, because the signal was split between the TR8818 and the oscilloscope. Although the oscilloscope's input impedance was much larger than that of the TR8818, $1 \mathrm{M} \Omega$ compared to $50 \Omega$, the duration of the MCP's output pulse was much less than the time required to reach the oscilloscope. Therefore, the fraction of the signal that started down the cable was determined by the $50 \Omega$ characteristic impedance of the cable, not by the impedance at the end. The system gains in normal operation should then be twice those that were measured. I tested the validity of this correction by compiling histograms of measurements that were taken with trigger signals derived from the analog output of the TR8818. This output is a representation of the input signal after it has passed through the analog-to-digital converter and then a digital-to-analog converter, and it is very noisy. The noise was partly overcome by placing a times-ten amplifier at the TR8818 input, but it was still impossible to trigger on most of the input pulses that produced low readings in the digital output. After extrapolation of the histogram to zero, the average output was $88 \mathrm{mV}$ per detected electron, which would have been $8.8 \mathrm{mV}$ per electron without the amplifier, at $2.209 \mathrm{kV}$ applied voltage. Even though this number contains a large factor (1.7) from the extrapolation, it is close enough to the value of $7.2 \mathrm{mV}$ from the other measurements to indicate that the splitting factor was closer to two than to $10^{4}$. 


\section{Measurement of subsystem gains}

I measured the pulse height distribution from the MCP alone by photographing oscilloscope traces, again using the RGA as a source of electrons. These measurements provided a test that the electrons were arriving one at a time. Together with measurements by Dr. George Kyrala and Jan Studebaker of the TR8818's response to short pulses, they account for the observed system gain. I first photographed 131 pulses with $2.439 \mathrm{kV}$ applied to the divider chain, $0.3 \mathrm{~A}$ through the solenoid, and the usual current through the Helmholtz coils. The purpose of the solenoid current was to raise the count rate to a convenient level. The resulting histogram appears as the upper curve in Fig. 26. This curve appeared to have two peaks as well as a strong exponential component. I then collected 52 more pulses with peaks above $160 \mathrm{mV}$, using the same voltage but no solenoid or Helmholtz coil currents (lower curve of Fig. 26). These readings have a single peak. This shows that the solenoid field was bringing electrons to the MCP in pairs. Since there was no direct path from the RGA to the MCP, most of the electrons in the drift tube therefore were probably secondary electrons formed in collisions with the walls. The solenoid field would keep these secondaries together, so that whenever one struck the MCP, the other was likely to strike it also.

By the time a set of measurements was written into a data file in the computer, the distribution of pulse heights from the MCP had been modified by the TR8818. The TR8818 operated by sampling the applied voltage for $5 \mathrm{nsec}$, then holding its reading for $5 \mathrm{nsec}$ while digitizing it. If the input was a pulse of $2 \mathrm{nsec}$ FWHM, the value read depended upon where in the cycle the pulse had arrived. This dependence was tested with pulses of $160 \mathrm{mV}$ height, after attenuation, and $1 \mathrm{nsec}$ FWHM from a pulse generator. The pulse generator and the digitizer were run from a common external clock so that the pulses could be delivered at any chosen time in the digitizer's cycle. The measured output of the 


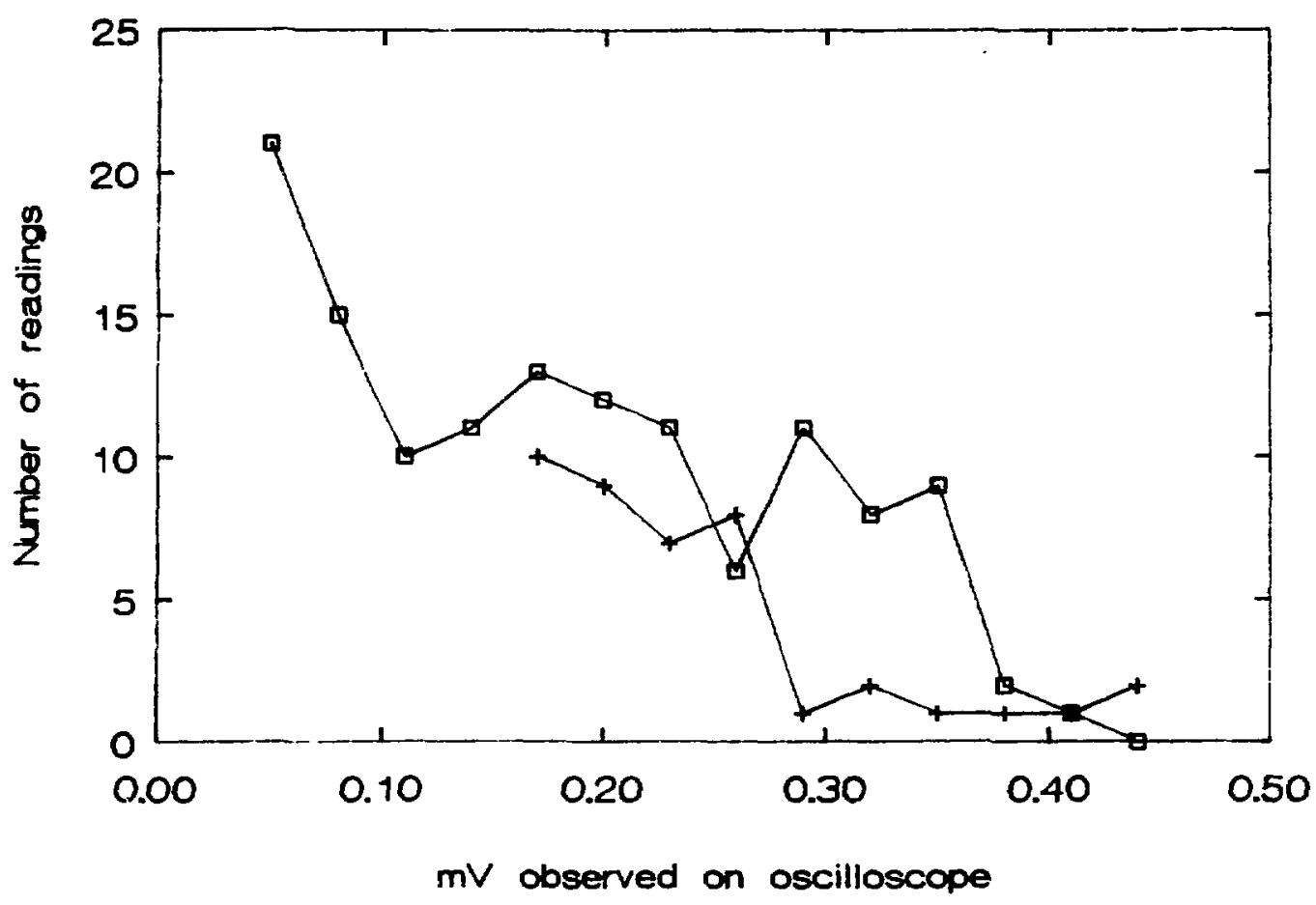

Figure 26. Distribution of MCP pulse heights read from oscilloscope traces. The curve marked with squares was collected with a solenoid current of $0.3 \mathrm{~A}$, the other curve with no solenoid current. The double peak shows that many electrons arrived in pairs when the solenoid was operating.

digitizer appears in Fig. 27. It can be converted to a histogram (Fig. 28). The exponential shape of this histogram in the case of uniform input pulses explains why the peak structure of Fig. 26 is not apparent in Fig. 25. 


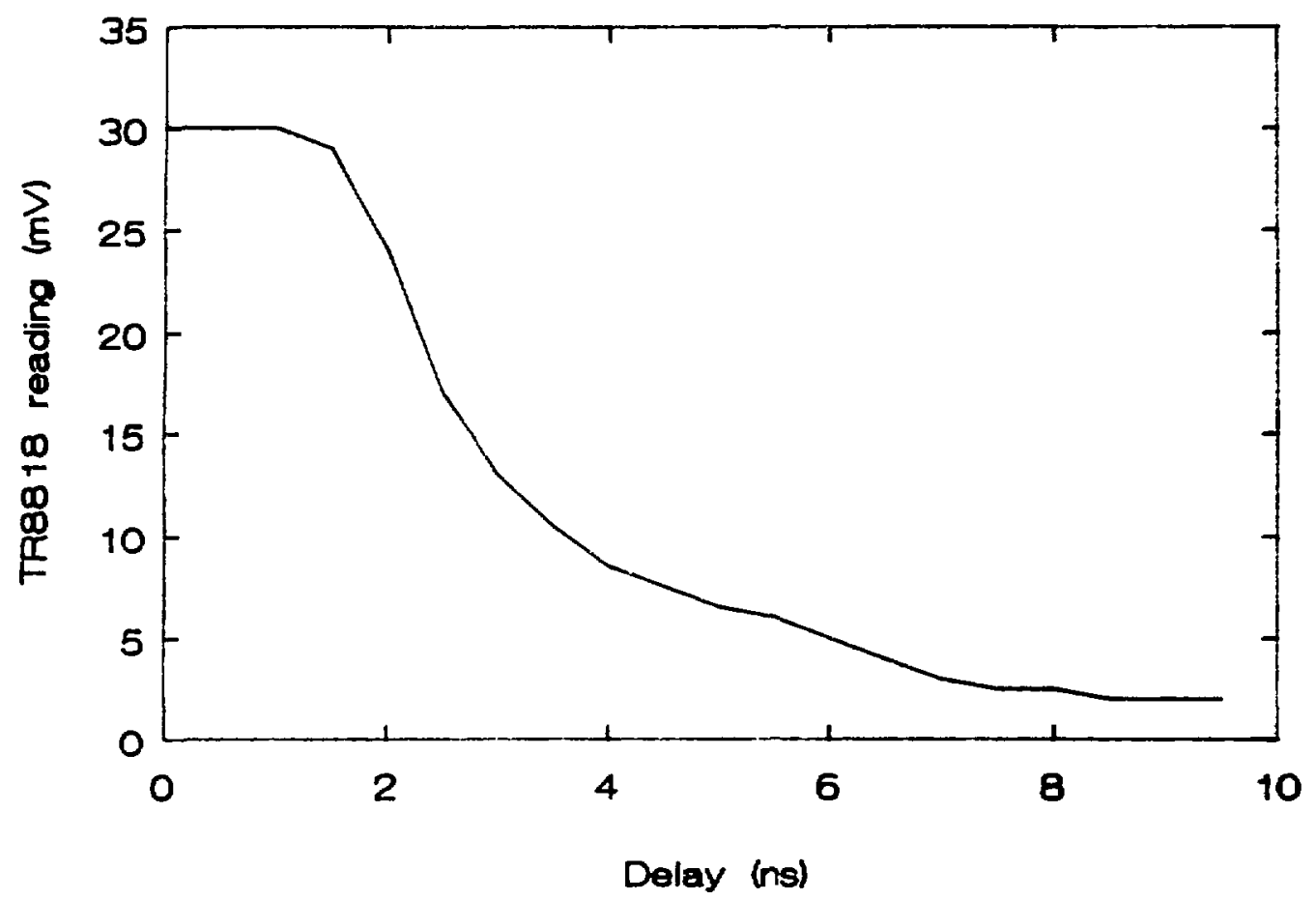

Figure 27. Response of the transient digitizer to short pulses arriving at different times in the cycle of operation. The input pulses were $160 \mathrm{mV}$ high and $1 \mathrm{nsec}$ wide.

\section{Comparison to manufacturer's specifications}

The manufacturer's data sheets for the individual microchannel plates listed gains at 700 and $900 \mathrm{~V}$ per plate as 370 and 4300 for one plate, and 370 and 4000 for the other. Assuming that each gain curve was exponential in voltage, and that the gain of the pair was the product of the individual gains, the total gains at three applied voltages were as listed in Table 17. A gain of $G$ means that one detected electron, of charge $e=1.6 \times 10^{-19} \mathrm{C}$, produces an average output charge of Ge. The TR8818 had an input resistance of $R_{i}=50 \Omega=50 \mathrm{~V} \mathrm{~s}^{-1}$ 


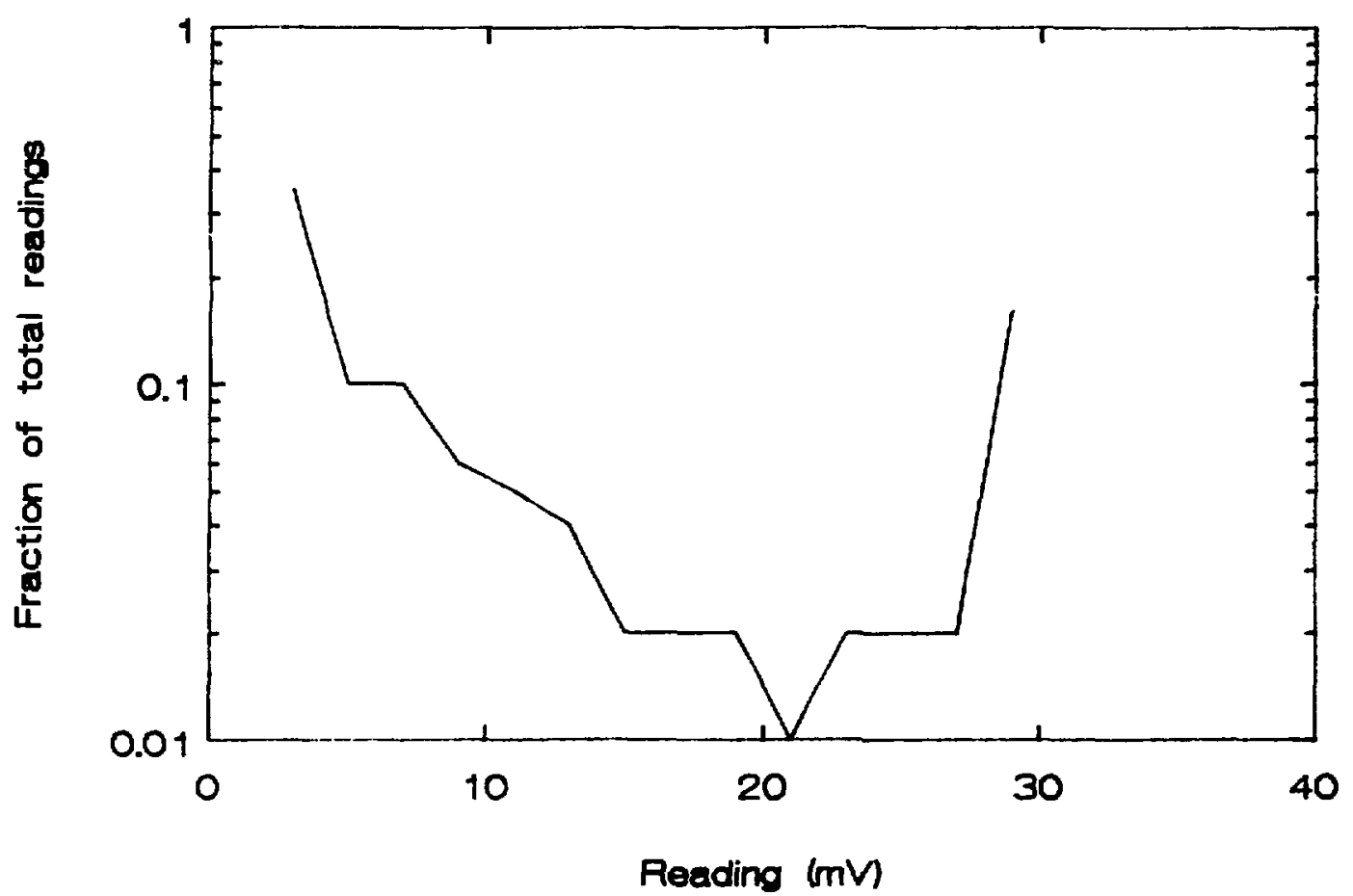

Figure 28. Histogram of the response of the transient digitizer to short pulses arriving at different times in the cycle of operation.

and an average output of $O_{a}=0.074 \mathrm{mV}$ per $\mathrm{mV}$ nsec detected. The expected output $O_{\mathrm{e}}$ from a single detected electron at gain $G$ was thus

$$
O_{e}=G e R_{i} O_{a}
$$

Values of $G$ and $O_{e}$ appear in Table 17 with measured values of TR8818 output from Table 16. The calculated responses were in the right range, but they increased more quickly with voltage. 
Table 17. Calculated MCP gain and TR8818 output compared to measured TR8818 output. The measured output produced by a single detected electron is numerically equal to the MCP system gain $G_{M}$.

\begin{tabular}{ccccc}
\hline \hline $\begin{array}{c}\text { Supply } \\
\text { voltage } \\
(\mathrm{kV})\end{array}$ & $\begin{array}{c}\text { Volts per } \\
\text { plate } \\
(\mathrm{V})\end{array}$ & $\begin{array}{c}\text { Calculated } \\
\text { gain G } \\
\text { (millions) }\end{array}$ & $\begin{array}{c}\text { Calculated } \\
\text { output } O_{e} \\
(\mathrm{mV})\end{array}$ & $\begin{array}{c}\text { Measured } \\
\text { output } \\
(\mathrm{mV})\end{array}$ \\
\hline 2.209 & 888 & 12.93 & 7.7 & 7.2 \\
2.309 & 928 & 34.18 & 20.2 & 11.2 \\
2.409 & 969 & 90.31 & 53.5 & 17.0 \\
\hline \hline
\end{tabular}

\section{Additional observations}

Figure 25 contains two large excursions from the average curve. These represent values of TR8818 output that were systematically shifted downward by one unit, from 16 to 15 and from 32 to 31 . This resulted in extra readings of 26 and $58 \mathrm{mV}$, and too few readings of 28 and $60 \mathrm{mV}$, where one unit represents $2 \mathrm{mV}$, and an offset register in the TR8818 was set to produce two units of output when the input signal was zero. The serial number of this TR8818 unit was A00109, and the serial number of the model MM8103A memory unit used with it was A14089. On another occasion, a different offset was set in the TR8818, so that a different reading in millivolts corresponded to each number of units. The missing readings again corresponded to 16 and 32 units. (A few readings appear at $28 \mathrm{mV}$ in Fig. 25. These result from a zero-level subtraction in the computer program which occasionally adjusted the reported values by one unit.) Since the readings were only shifted, not lost, this behavior did not affect electron spectra significantly.

The output voltage from the power supply was very stable. I tested it by applying $1.609 \mathrm{kV}$ to the resistor chain and measuring the ground-to-first-plate voltage with a Keithley model 617 programmable electrometer. A computer program read the electrometer over a GPIB circuit every ten seconds for over 
fourteen hours on 26Jun90 and recorded the readings. The highest and lowest readings during that time were 187.437 and $187.226 \mathrm{~V}$, the highest coming at the beginning, 9:40 a.m., and the lowest at 4:34 p.m. These voltages appeared to vary inversely with the temperature of the lab. From 7:25 p.m. to the end of data at 11:45 p.m., when the cooling system could be expected to hold the temperature within narrow limits, the readings oscillated between 187.29 and 187.31 V. The variation in MCP gain due to these measured changes in applied voltage would be $\pm 0.55 \%$ over the 14 -hour period.

The probability of two electrons striking the same channel of the MCP was very small. Since the gas density in the interaction region was reduced when high irradiances were used, and since fewer than half of the electrons from the interaction region entered the spectrometer and arrived at the MCP, there were never more than $10^{3}$ electrons in a pulse. These electrons were distributed over a magnified image of the interaction region that was approximately $0.6 \mathrm{~mm}$ high and $12 \mathrm{~mm}$ wide. At one channel per $156 \mu \mathrm{m}^{2}$, there were $4.6 \times 10^{4}$ channels available for $10^{3}$ electrons. Even allowing for a higher concentration at the center of the image, there was not much chance of any electron striking a depleted channel. 


\section{A ppendix E. Listings of computer programs}

The following programs, RUN, NEWRUN, ERUN, and DRUN, with the assembly language subroutines CONVERT1, DISCRIM, and the original CONVERT (which is not listed here), operated the equipment on the GPIB and CAMAC circuits, and stored processed data in disk files. The computer was an IBM PC-AT running DOS 3.30, and the language was Microsoft QuickBASIC 3.0. Subroutines called to operate the GPIB circuit were purchased with the controller board from National Instruments. ERUN and DRUN ran only within the QuickBASIC environment, while the others ran also as stand-alone programs.

1. RUN

This is the program used to collect electron spectra at the end of 1988 . The plotter output section, which is very similar to that of NEwrUN, has been omitted. The CONVERT subroutine, which is functionally identical to CONVERT1, has also been omitted.

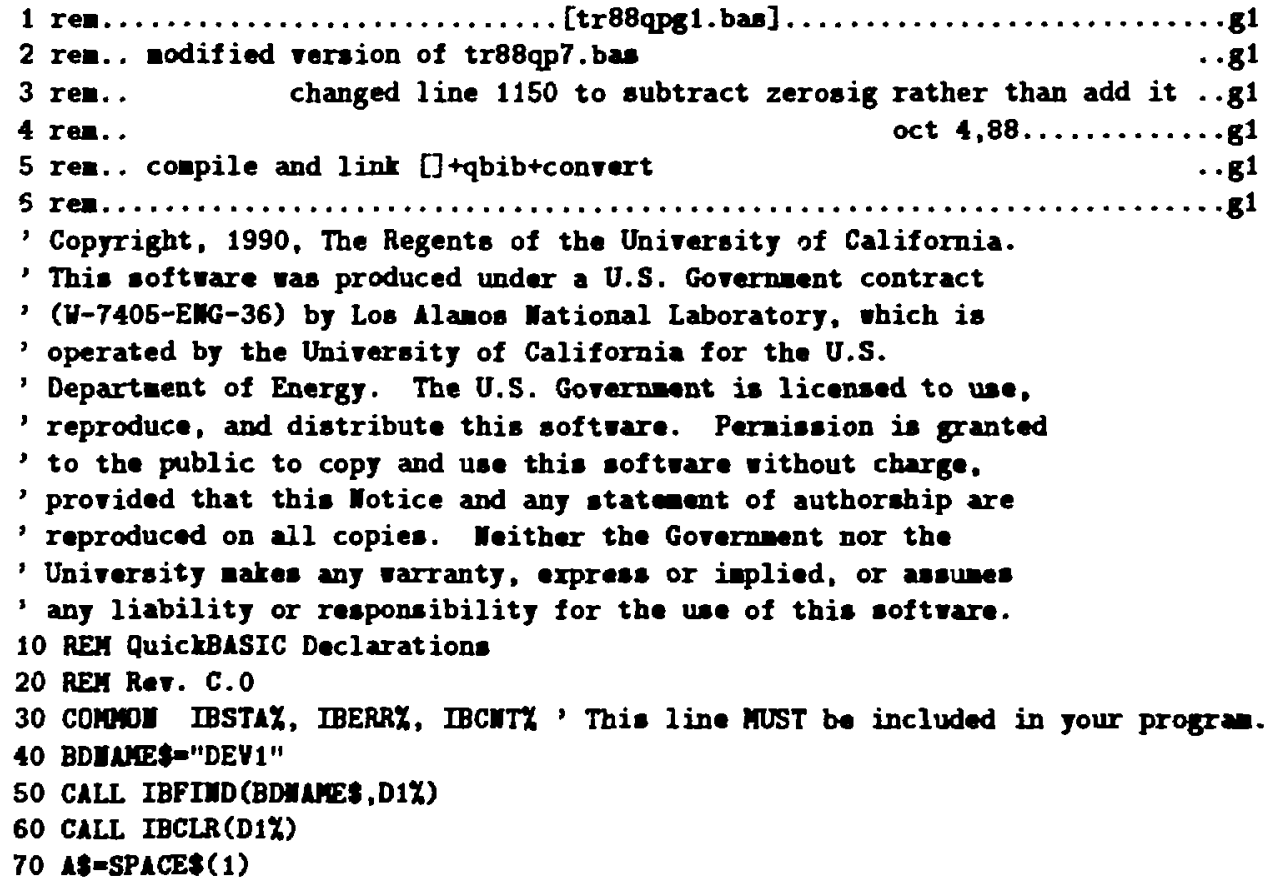




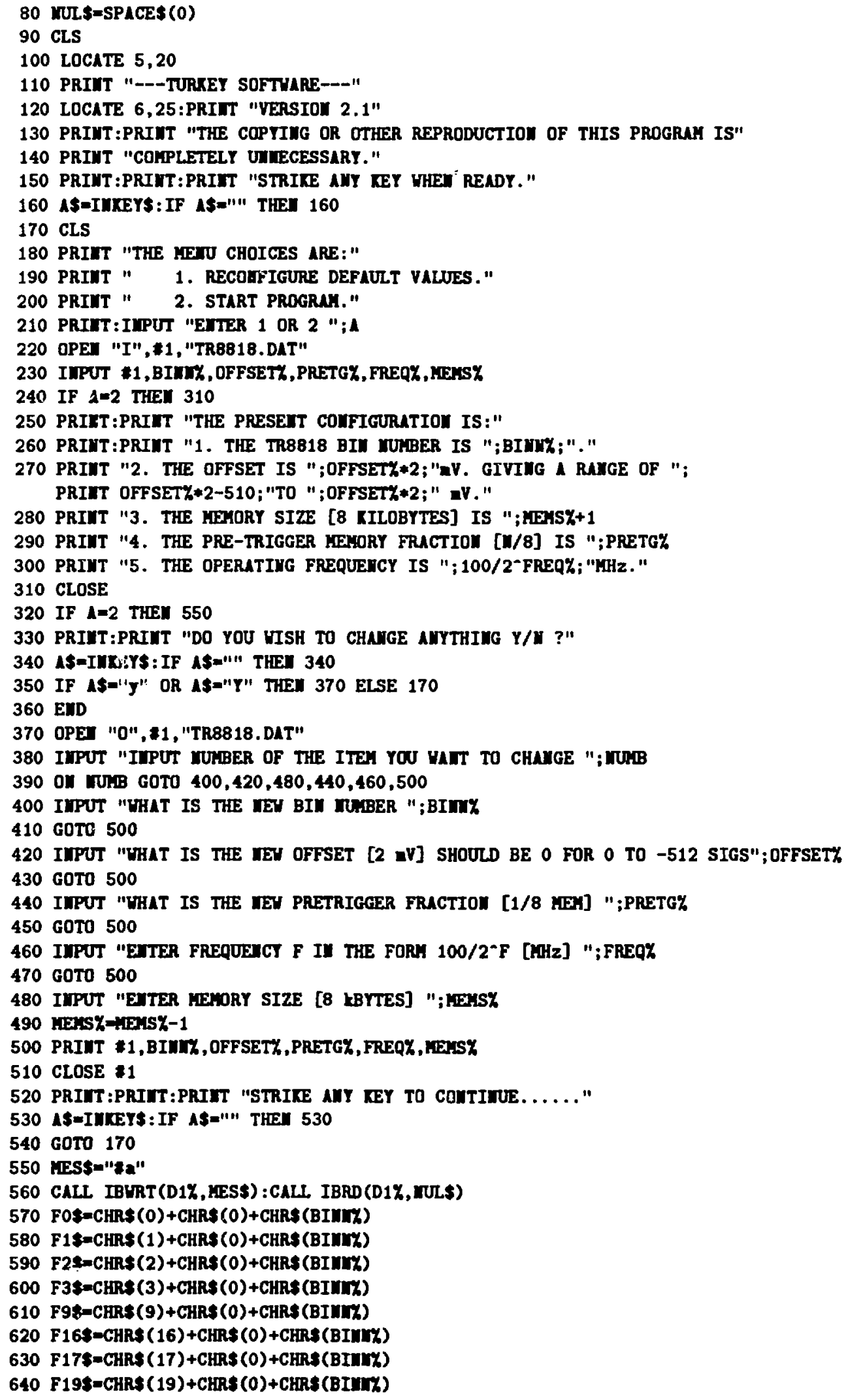




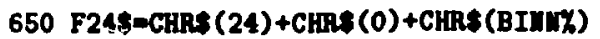

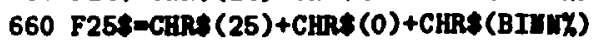

670 F265-CHRs (26) +CHRs (0) + CHRs (BIMNY)

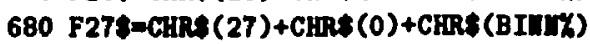

690 F10 - -CHRS $(10)+$ ChRS (0)+CHRs (BIIIY)

700 REX SDYHNIC

710 DIH DTA (2500), X(2500)

720 REA SSTATIC

730 DII IX(4200), DATK(8200)

$740 \quad L G \%=2500$

750 B1=PREQ $\% * 16+$ PRETG $\%$

760 B2-IFMis\%

770 CALL IBLRT (D1\%,F10\$) : CALL IBRD(D1\%, IULS)

780 F\$ F 16\$+CHR\$(B1)+CHR\$(B2)

790 CALI IBLRT(D1\%,F\$) :CALL IBRD(D1\%,ITL\$)

800 FS-F19\$+CHRS (OFFSETK)

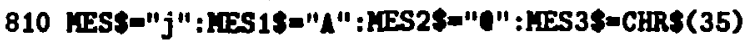

820 LASTK= $=44800$

830 ODR\%-VARPTR(DAT\%(0))

840 CAII IBURT (D1\%,F : CALL IBRD(D1\%, NULS)

850 CALL IBLRT (D1\%,F26\$) :CALL IBRD(D1\%, MUL\$)

860 COUIT $=0$

870 CLS:PRIIT "STRIKE S TO EXIT DATA TAKIMG AT ANY TIKE", "COUIT YOU $=$; COUNT 880 IIPUT "EUTER MUMBER OF SHOTS OR ZERO FOR COMTIMUOUS DATA TAKIMG";SCOUIT 890 PRIUT:PRIIT " DATA ACQUISITION MOH TAKING PLACE."

900 CALL. IBURT (D1\%, MES3\$) : CALL IBRD(D1\%, IULS)

910 CALL IBLRT (D1\%,F26\$) :CALL IBRD(D1\%, JUL\$)

920 CALL IBLRT (D1\%,F9\$) : CALL IBRD(D1\%, UULS)

930 CAII IBURT (D1\%, NBS1\$)

940 CALL IBUAIT(D1\%,HAST\%) : CALL IBRD(D1\%,NUL\$)

950 CLLL IBRSP (D1\%,SPR\%)

960 CALL IBLRT (D1\%, HBS2\$)

970 CALL IBLRT (D1K,F10\$) : CALL. IBRD(D1\%, MUL\$)

980 CALL IBURT (D1\%,MES1\$)

$990 \mathrm{CALL}$ IBHAIT(D1\%,XAST) : CAII IBRD(D1\%,MUL)

1000 CALL IBURT(D1\%, IES2\$)

1010 CALL IBRSP(D1\%,SPR\%)

1020 CALL IBLRT (D1\%,F17\$) : CALL IBRD(D1\%, UUL $\$$ )

1030 CALL IBLRT(D1\%, ,ESS : CALL IBRD (D1\%, WUL\$)

1040 CALL IBLRT (D1\%,F2\$) : CALL IBRDI(D1\%,I\%(0),LG\%)

$1050 X$ XEEK (VARPTR(I\%(0))) AND 1

1060 ADRZ $=$ YRPTR. $(I K(0))+2+X$

1070 CONTT=COUIT +1

1080 LOCATE 10,30:PRIUT COUIT

1090 CALL COIUERT (ADR\%, ODR\%,LG\%)

1100 IF COJT-SCOUTT THEW 1120

1110 At-IITEYS:IF AS="S" OR AS="8" THEY 1120 ELSE 900

1120 DEIT=2-FREQ\% *10:ZEROSIG-OFFSETK*2

1130 FOR Q=0 TO LOY

1140 If $\operatorname{DATK}(Q)<0$ THEX DTA $(Q)=D A T K(Q)+65536$ ! ELSE DTA $(Q)=D A T K(Q)$

1150 DTA (Q) $=(-2 A E R O S I G+2 * D T A(Q) / C O U I T)$

$1160 x(Q)=Q$ DELT $/ 1000$

1170 जसT Q

1180 CLS

1190 PRIIT "HOU DO YOU UATT THE DATA OUTPUT ?"

1200 PRIII " 1. PLACED IN A DIST FILE "

1210 PRIIT " 2. PRIITED ON ThE PRIITER "

1220 PRINT " 3. PRIUTED ON THE SCREEM" 


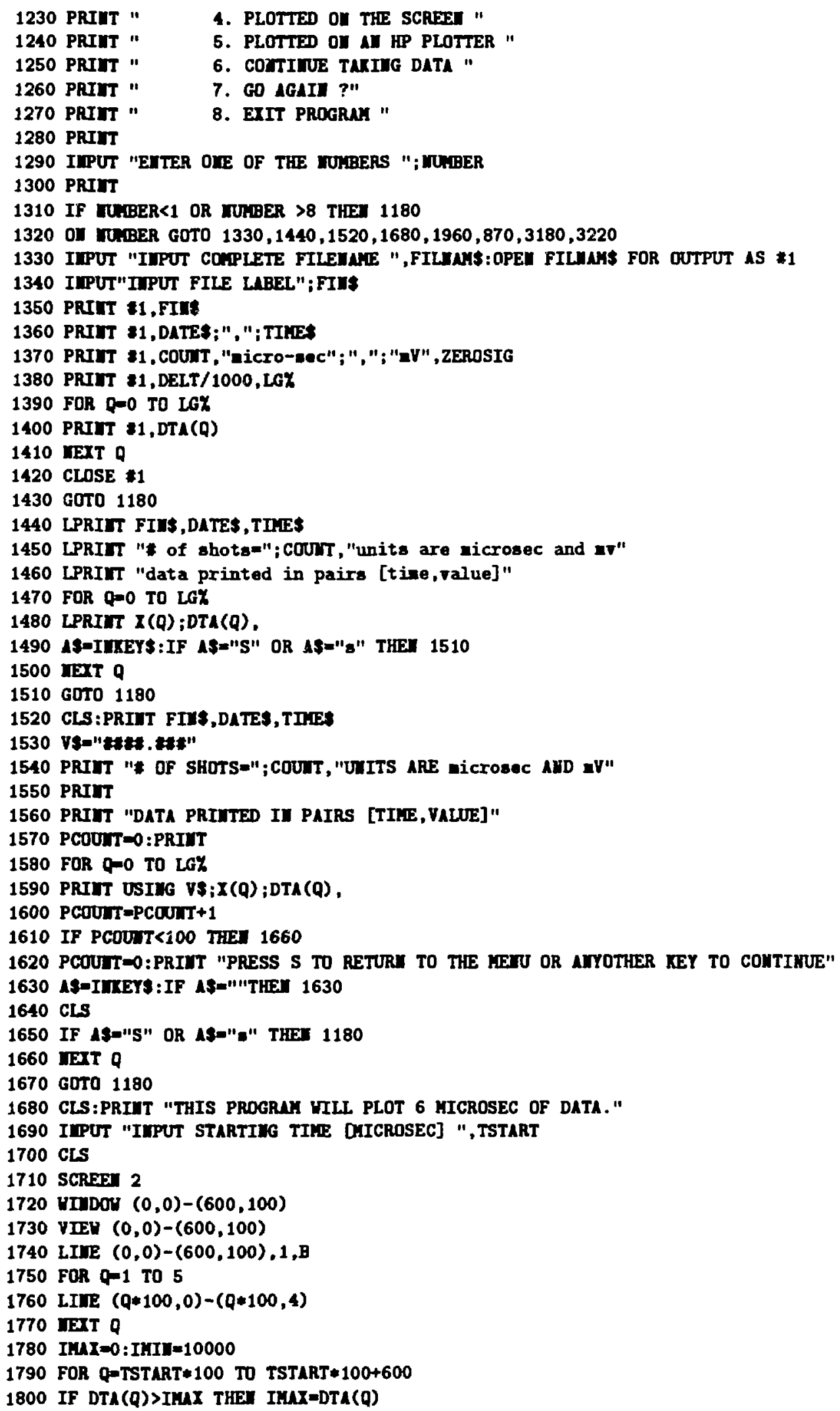




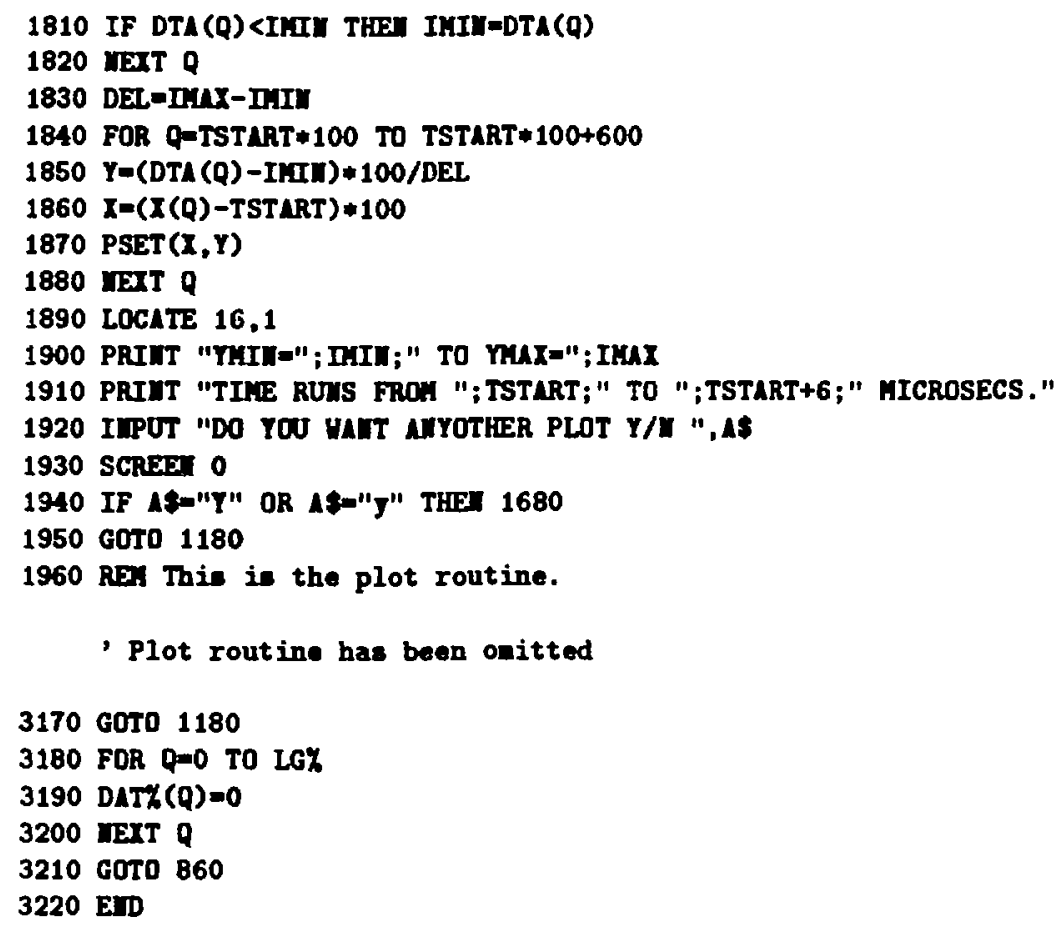

\section{NEWRUN}

This program serves the same function as RUN, but it avoids sending unnecessary commands to the TR8818 transient digitizer. Other improvements include protection against overwriting a previous data file, optional sequential names for data files, review of stored data, and comparison of newly collected data to stored data.

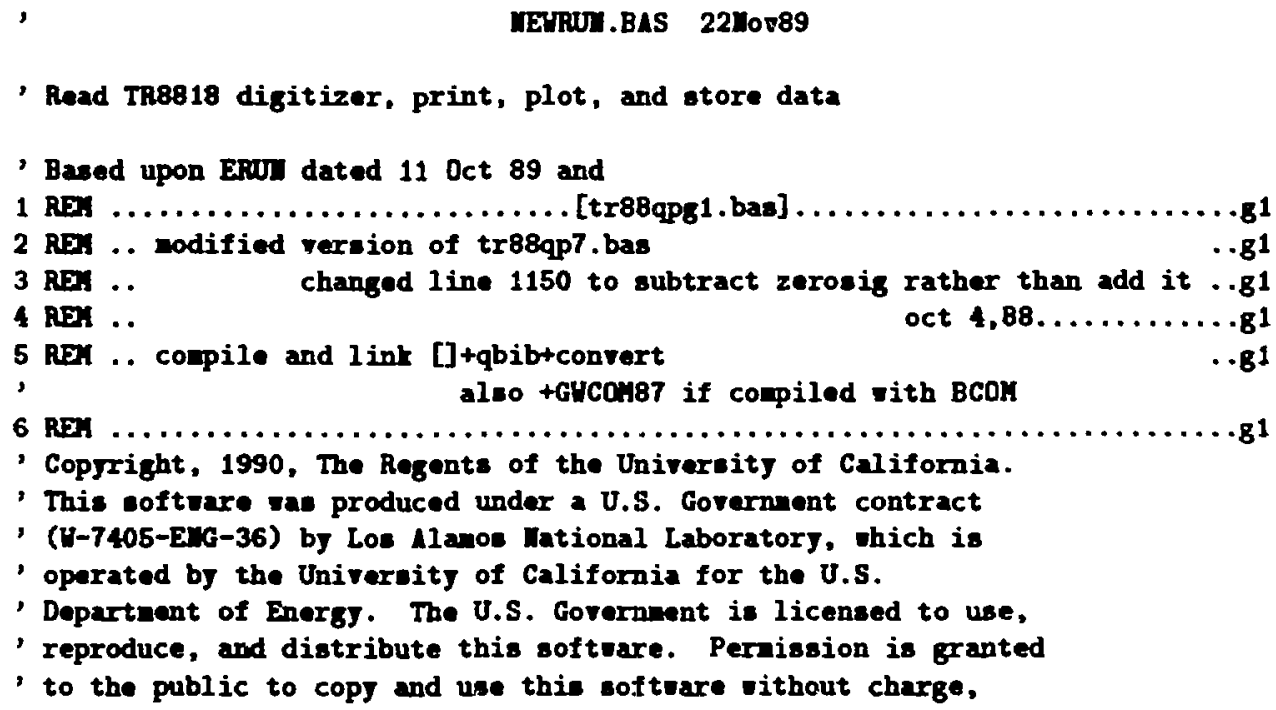




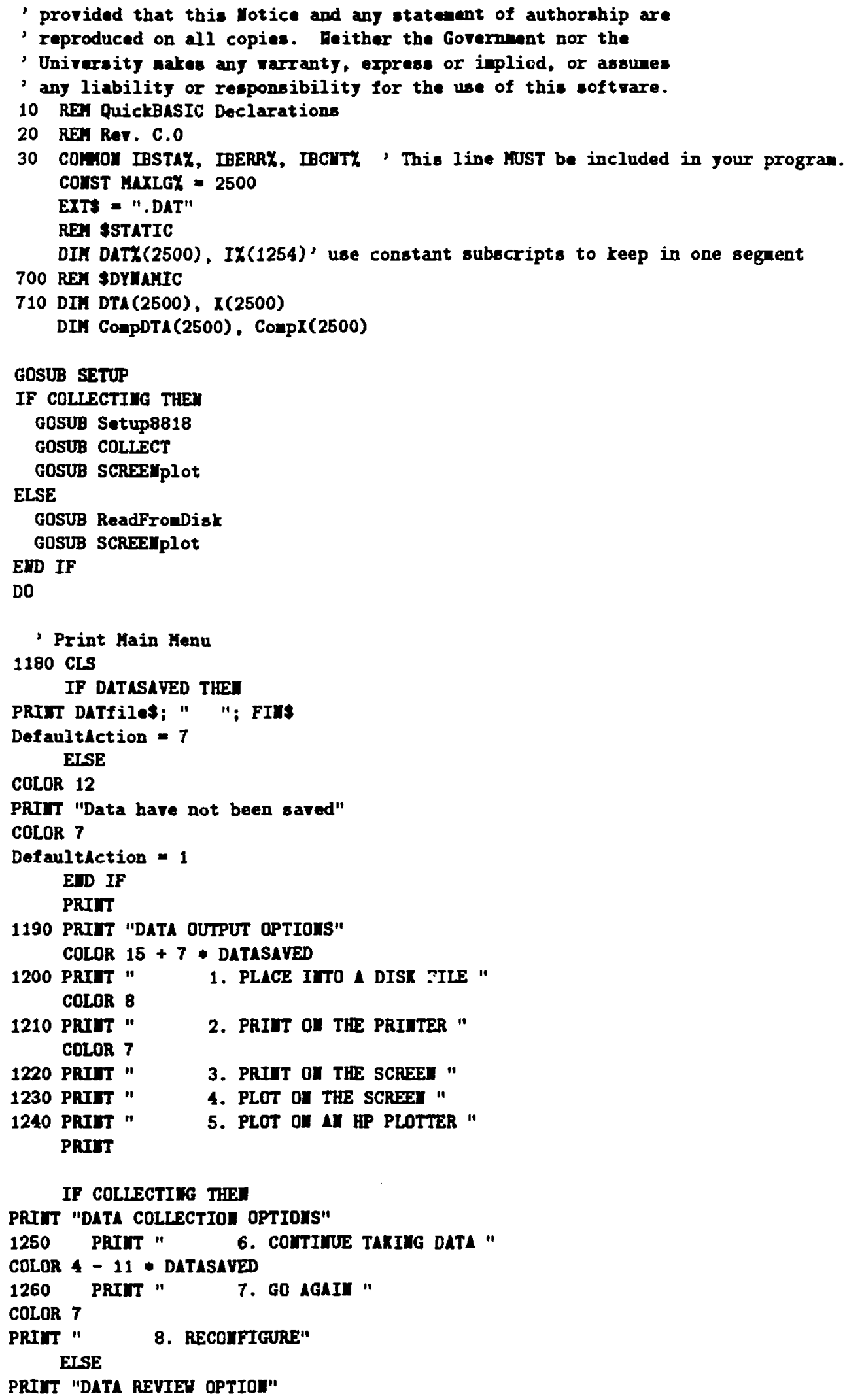




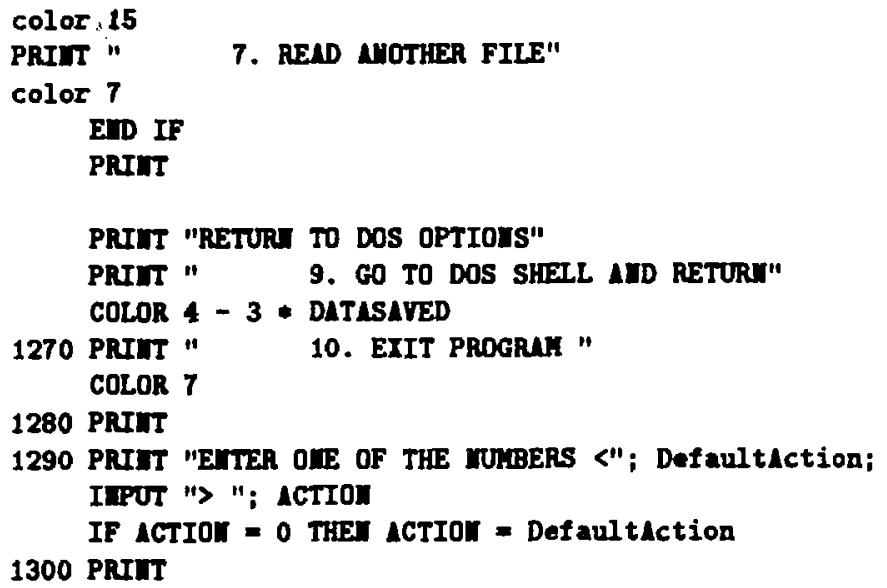




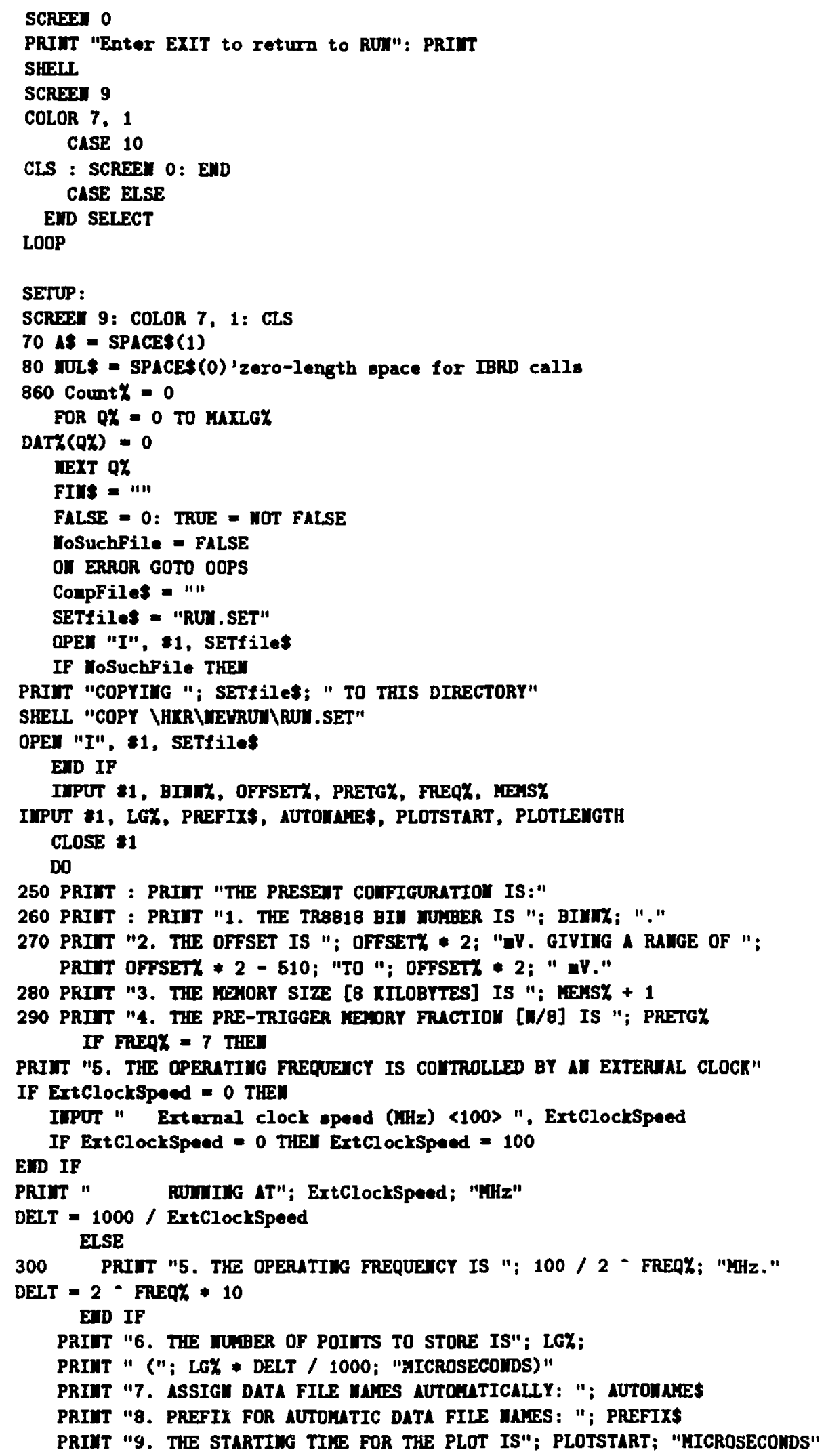




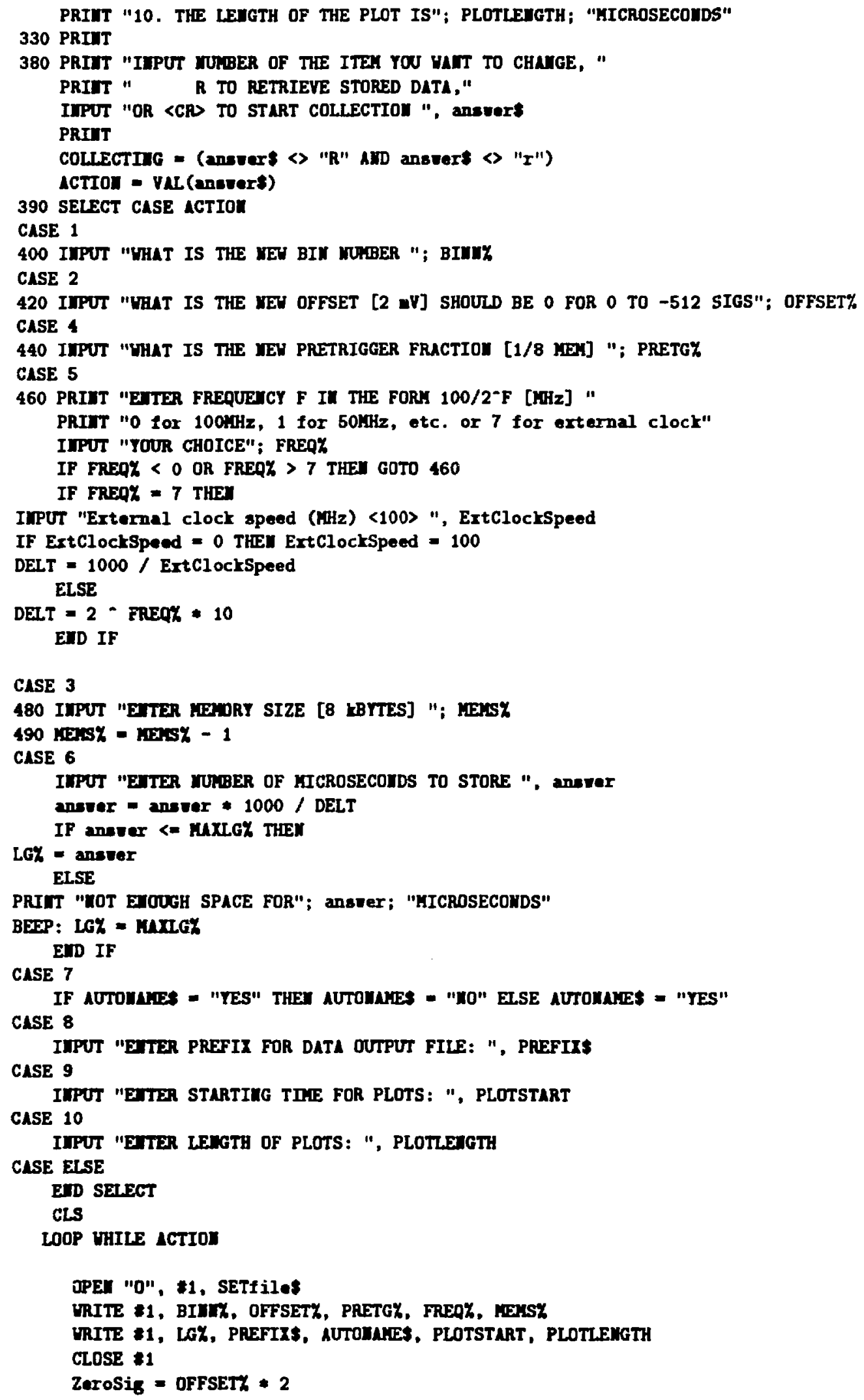


AUTONAYE = (AUTOMAKES $=$ "YES")

FOR QY $=0$ TO LGK

$X(Q \mathrm{X})=Q \mathrm{Q}$ * DELT / 1000

IEIT Q\%

RETUR

Setup 8818 :

40 BDULYEs - "DEV1"

50 CALL IBFIND(BDMANE\$, D1\%)

60 CALI. IBCLR(D1X)

' MES variables are connands to the 89014 GPIB Interface, ohich is

' the CAMC controller. Its nane is DEV1, and its address is D1\%.

- The PC2A board in the conputer clears the GPIB line autonatically

' at the first call aftar porerup. IBCLR sends SDC (selected device

, clear) to the 8901h, but the 89014 manual lists no response to it.

89014 comands are

, $\%$ = CHR $\$(35)$ send clear and initialize (CAMAC 2 ) to all CAHAC devices

a 8-bit read, normal transfer

, j 16-bit high speed block read (on conpletion of the block

read, the state becones 16-bit read, normal transfer)

$\boldsymbol{\Lambda}$

enable SRQ on LAH

- disable all SRA's

- These comands are sent by IBURT. They take effect inediately - they

' do not require a CAMC cjcle

550 HESsendzs = "s"

810 HES16blockreads = "j"

MESenablesRnonichts = " $A$ "

MESdisablesRat = "Q"

- TRF rariables are $F$ comands to the IR8818 Transient Recorder

0 - Read (send to controller) the pre-trigger sapple size, the sample period, and the active nenory size

1 - Recid the last single sanple taken

2 - Read vareform data in 16-bit vords

3 - Read nodule identifier

8 - Test LAH, setting $Q$ if LAM line is being asserted

9 - Set Readj State (begin digitizing)

10 - Clear LAH

16 - Urite (accept fran controller) ralues read by Fo

17 - Enable Read Mode (required before F1 or F2)

19 - Urite offset

24 - Disable LAH

25 - Conputer stop trigger

26 - Enable LM

' 27 - Test LAH, setting $Q$ on internal LAH vhether or not LAH is enabled

- These nust first be loaded into the controller (8901/ GPIB interface)

vith an IBURT, then sent to the TR8818 as the first part of a CAMC

cycle. The controller initiates a curuc cycle erery tine it is

" comanded to "talk" (wually by IBRD or IBRSP) unless it is aseerting

, SRQ on the GPIB. Every subequent CAHAC cjcle starts vith the

- F comand wost recently loaded, eren if it has been sent before.

- Jotice that jou cannot clear a CAMAC LAN while a GPIB SRQ is pending.

' You nust first disable "SRq on LAH", then clear the SRq vith IBRSP,

' then clear the LW, then re-enable the SRQ.

570 TRFOS $=\operatorname{CHR}(0)+\operatorname{CHR}(0)+\operatorname{CHRS}($ BIMIK) 


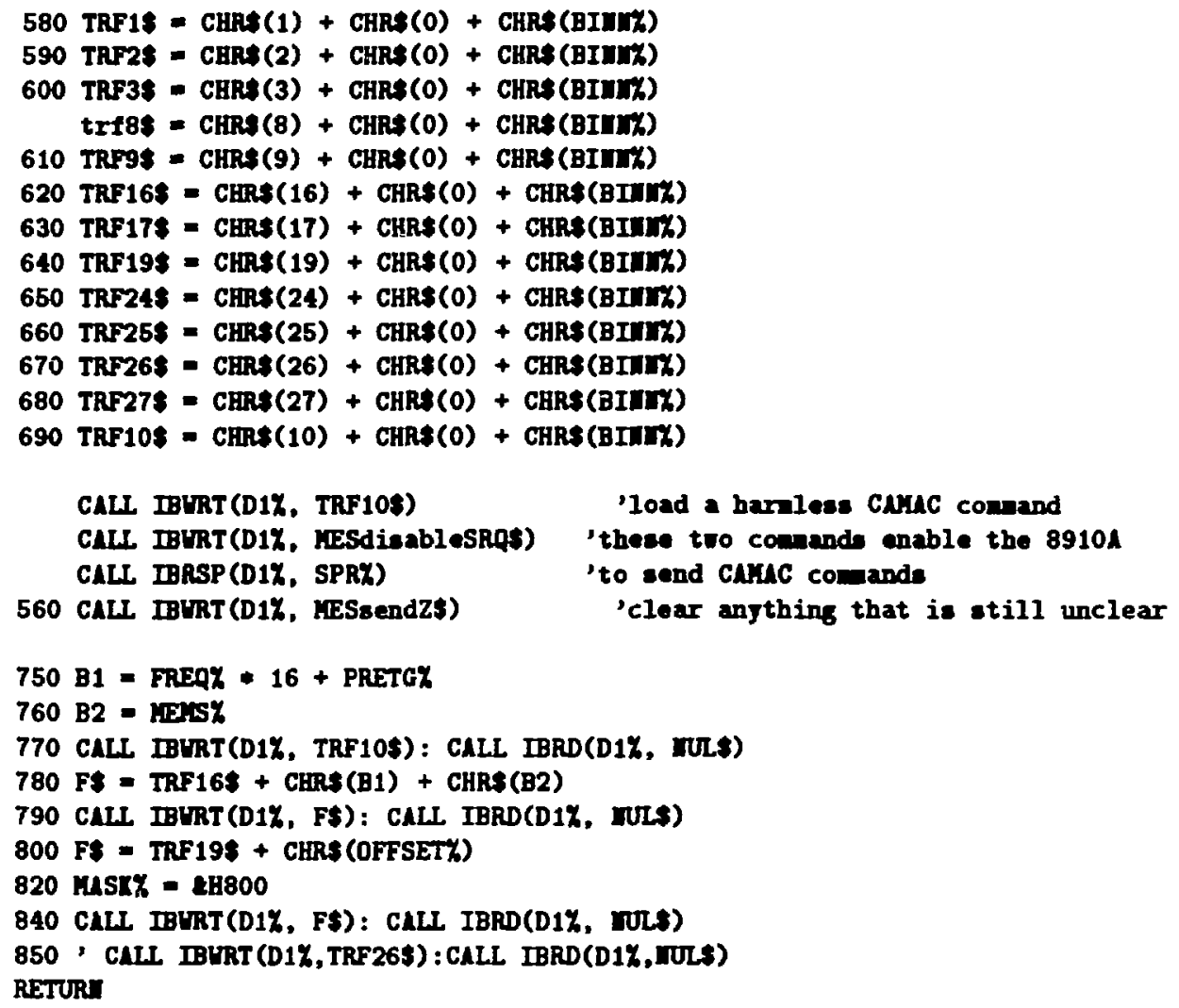

CALL IBURT (D1\%, TRF10\$)

CALL IBURT(D1\%, VESdieableSRG\$)

CALL IBRSP(D1\%, SPR\%)

560 CALL IBURT (D1\%, MESsendZ9)

'load a harnless Curuc comand

'these two comands enable the $8910 \mathrm{~A}$

'to send curhe comands

'clear anjthing that is still unclear

750 B1 = FREQY * $16+$ PRETGY

$760 \mathrm{B2}=$ MENSY

770 CALL IBURT(D1\%, TRF10\$): CALL IBRD(D1\%, WUL\$)

$780 \mathrm{~F} \$=\operatorname{TRF} 16 \$+$ CHR $\$$ (B1) + CHR $\$$ (B2)

790 CALL IBURT (D1\%, F\$): CALL IBRD(D1X, IUL\$)

800 F\$ - TRF19\$ + CHR (OFFSET\%)

820 MASKY $=$ -

840 CALI IBURT(D1\%, F\$): CALI IBRD(D1\%, IUL\$)

850 ' CALL IBURT(D1\%, TRF26\$) : CALL IBRD(D1\%, TUL\$)

RETURI

COLLECT:

CLS

870 PRIT "STRIKE O OR S TO EXIT DLTA TAYIM AT AIT TIE", "COUTT YOV ="; Count\%

880 IMPUT "EITER IURBER OF SHOTS OR ZERO FOR COMTINUOUS DATA TAXIIG"; SCOUIT

890 PRIMT : PRITT " DATA ACQUISITIOI WOU TAKIIIG PLACE."

LOCATE 8, 35: PAIIT "COUW"

IF Count $Y=0$ THEW STARTDATE\$ = DATES: STARTTIME\$ = TTES

DATASAVED $=$ FALSE

GOLGAII - FALSE

'Start IRB818, test for internal LWI without inrolving the GPIB SRO

'Throw out the first set in case the stop trigger cane before a conplete

'collection. Progran is then esnchroaized to the laser.

CALL IBLRT(D1\%, TRF98): GOSUB VaitGPIB 'start digitizing

CALL IBISP (D1\%, SPRY): GOSUB VaitGPIB

'IF SPAX<>1 THEI PAIT "FAILED TO START TR8818 - STATUS "; HEX\$(SPRY)

CALL IBURT(D1\%. TRF27\$): GOSUB VaitGPIB 'test internal LAH

DO

CALI IBRSP(D1\%, SPRY) : GOSUB YaitGPIB

LOOP UITIL SPR\% $=3$

CALI IBURT(D1\%, TRF9\$): GOSUB VaitGPIB 'start digitizing

CALL IBRSP(D1\%, SPRY): GOSUB WaitGPIB

'IF SPRX<>1 THEI PRITT "FAILED TO START TRA818 - STATUS "; HEXS(SPRY)

CALL IBURT(D1\%, TRF27\$) : GOSUB VaitGPIB 'test internal LAH

DO 'this starts the COUIT loop 
'Teat for internal wal in the correct CAMC unit,

' using previously stored TRF27's comand Do

CALI IBRSP(D1\%, SPRY): GOSUB HaitGPIB

LOOP UTTIL SPRY $=3$

'Read TR8818 data

'PRIUT "LAY DETECTED"; : GOSUB PRESSIEY

CALL IBLRT(D1\%, TRF108): GOSUB HaitGPIB 'clear LAH

CALL IBRD(D1\%, IULS): GOSUB VaitGPIB

'PRINT "LAY CLEARED"; : GOSUB PRESSKEY

CALL IBLRT(D1\%, TRF17\$): GOSUB YaitGPIB 'enable readout

CALL IBRSP(D1Y, SPRY): GOSUB YaitGPIB

'PRITT "TRF17"; : GOSUB PRESSXEY

CALL IBURT(D1\%, MES16blockreads): GOSUB HaitGPIB 'bigh speed 16 bit block read

CALL IBURT(D1\%, TRF2\$): GOSUB HaitGPIB 'start readout

CALL IBRDI(D1\%, IK(0), LGK + 4): GOSUB UaitGPIB 'read data to IK array

'Start TR8818 so it can collect the nert point vhile the progran runs

'Load a safe coanand into the CAruc instruction register

CALI IBURT(D1\%, TRF9\$): GOSUB YaitGPIB

CLLL IBRSP(D1\%, SPRY) : GOSUB YaitGPIB

'IF SPRY<>1 THEM PRIUT "PAILED TO START TR8818 - STATUS "; HEXS(SPRY)

CALL IBURT(D1\%, TRF27\$): GOSUB UaitGPIB 'test internal LAH

- Unpack values and add to DAT array

$1050 X$ = PEET (VLRPTR(IK(0))) MID 1

$1060 \triangle D A Y=V A R P T R(I Z(0))+2+X$

830 ODR\% = VARPTR(DATY (0))

1070 Count $\%$ - Count $\%+1$

1080 LOCATE 10, 35: PRIIT Count\%

1090 CALL COIVERT (ADR\%, ODR\%, LGK + 1)

LOOP UTIL (Count\% = SCOUNTY) OR (IMKEYS $=$ "0") OR (IMKEYS = "S") OR (IMKEYS $=$ "s")

STOPTIRES = TIRE

- Convert values to $\mathrm{m}$ and load into DTA array

FOR $Q \%=0$ TO LGK

IF DATK (QX) < 0 THEI

$\operatorname{DTA}(Q K)=\operatorname{DATK}(Q \%)+65536$ !

ELSE

$\operatorname{DTA}($ QY) $=\operatorname{DATK}(Q \%)$

EID IF

DTA (QY) - -ZoroSig + 2 - DTA (QY) / Count

IETT a\%

BEEP

RETURU

PRESSKEY :

PRIIT "PRESS ATT KEY TO COITITUE"

DO

LOOP HHILE IMKEY = "'"

RETUR

ReadFronDisk:

DATASAVED $=$ TRUE

DO 


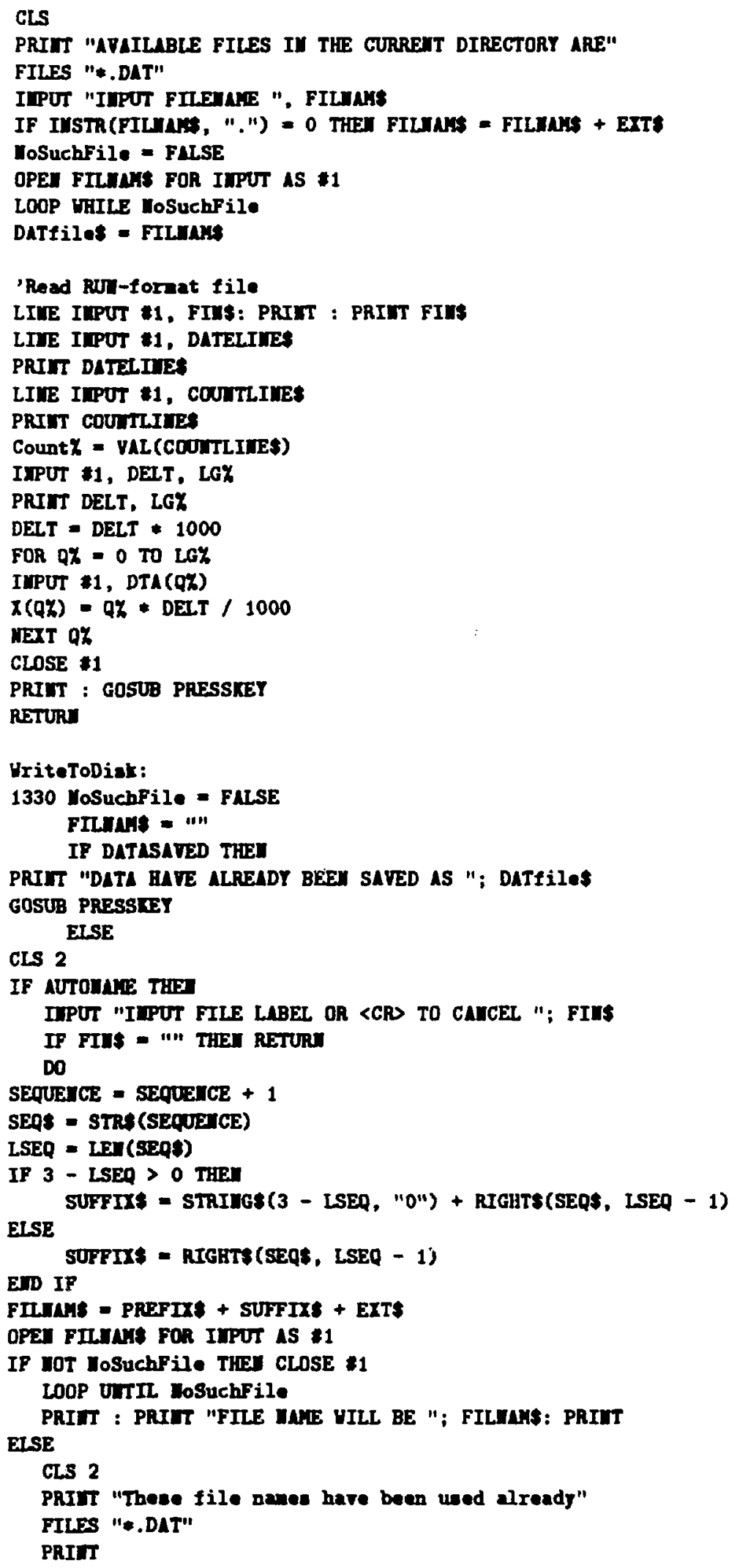




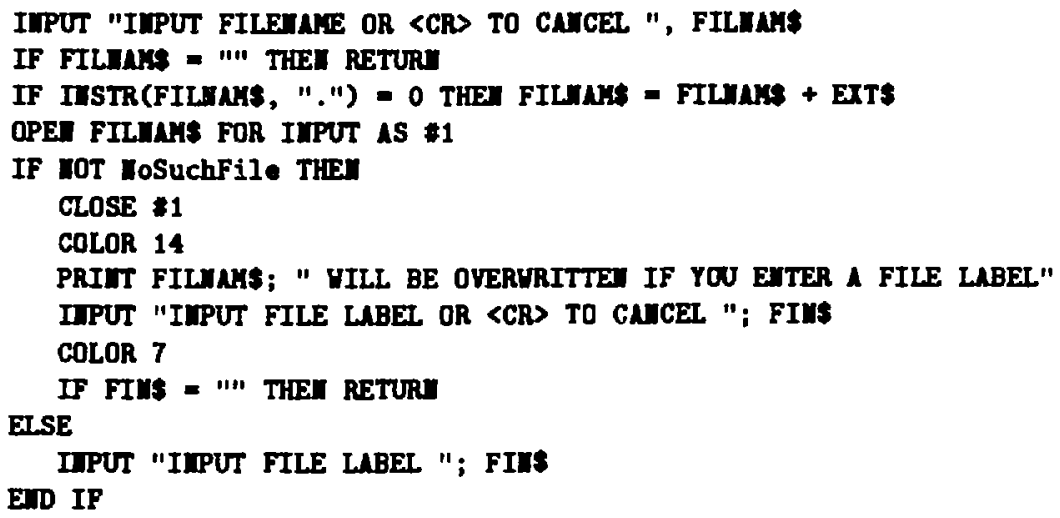

\section{SCREETPUIHT:}

1520 CLS : PRIIT FIIS, STARTDATES, STARTTIKES

1530 V\$ = "tatt*tat"

1540 PRIT " OF SHOTS"; Count\%, "UIITS ARE nicrosec AID EV"

1550 PRIIT

1560 PRIIT "DATA PRIUTED IU PAIRS [TIKE, VALUE]"

1570 PCOUIT $=0:$ PRIMT

$1580 \mathrm{FOn} \mathrm{QK}=0$ TO LGK

1590 PRINT USInG vs; $X(Q \%)$; DTA $(Q \%)$;

1600 PCOUII $=$ PCOUITY +1

1610 IF PCOUTT < 100 THF्य 1660

1620 PCOUIY $=0$ 
PRIIT "PRESS O OR S TO RETURN TO THE FENU, OR ATY OTHER KEY TO COITIMUE" 1630 AS = IMKETS : IF AS = "I" THEI 1630

1640 CLS

1650 IF As = "O" OR As - "S" OR As = "s" THEW 1670

1660 TEIT QY

1670 RETURI

SCREEIPlot:

TSTART = PLOTSTART

TLEMGTB = PLOTLEMGTH

DO

1700 CLS 0

1720 บIIDON $(0,0)-(600,200)$

1730 VIEU $(10,10)-(629,200)$

1740 LIIE $(0,0)-(600,200)$, , B

1750 FOR QY $=1$ TO TLEWGTH

1760 IIIE (Q\% * 600/ TLETGTH, 0)-(Q\% *600/ TLEMGTH, 6)' time tics

1770 MEXT QY

VIEU PRIIT 16 TO 25

PRIIT "AVERAGE OF"; Count\%; "SHOTS"; TAB(40);

1910 PRIUT "TIFE RUIS FROA "; TSTART; " TO "; TSTART + TLEMGTH; " MICROSECS."

1780 IMUI $=-10000:$ IMII $=10000$

QSTARTK $=$ TSTART $* 1000 /$ DELT

IF QSTARTK > LGK THEM QSTARTK = LG\%: PRIMT "START TIFE IS AFTER END OF DATA"

QSTOPY = (TSTART + TLENGTH) $* 1000 /$ DELT

IF QSTCPY > LGK THEW QSTOPY = LGK

1790 FOR QK = QSTARTK TO QSTOPY

1800 IF DTA (QY) > IMUX THEW IMAX = DTA(QY)

$1 B 10$ IF DTA(QY) < IMIN THEW IFIN = DTA(QZ)

1820 IEXT QY

$1830 \mathrm{DEL}=\mathrm{IMX}$ - IFII

IF DEI = 0 THEN

PRITT "All points in this range have value "; ImhX ELSE

DEL $=1.1 *$ DEL

1840 FOR QK = QSTARTK TO QSTOPK

$1860 \quad X=(X(Q Z)-T S T A R T) * 600 /$ TLENGTH

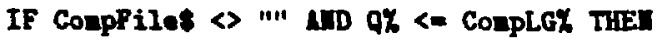

$Y=($ Compta (QY) - IIIII) $* 200 /$ DEI +10

$\operatorname{PSET}(x, Y), 12$

EID IF

$1850 \quad Y=$ (DTA (QY) - IMII) $* 200 /$ DEL +10

1870 PSET $(X, Y), 16$

1880 IEIT QK

1900 PAIIT "YIII-"; IRII; " TO MAX="; IMAx; TAB(40);

IF DATASAVED THE PRIIT DATfiles; " ";

COLOR 4: PRIJ COApFiles: COLOR 7

EDI IF

PRIIT

IF MOT DATASAVED THE PRIIT " 0 - Save Data"

COLOR 15

PRIIT "

1 - Main Menu"

COLOR 7

PRIMT "

PRIIT "

PRITT "

2 - Change Plot Limits"

PRIIT

3 - Paper Plot with Those Linits"

4 - Select Conparison Data" 


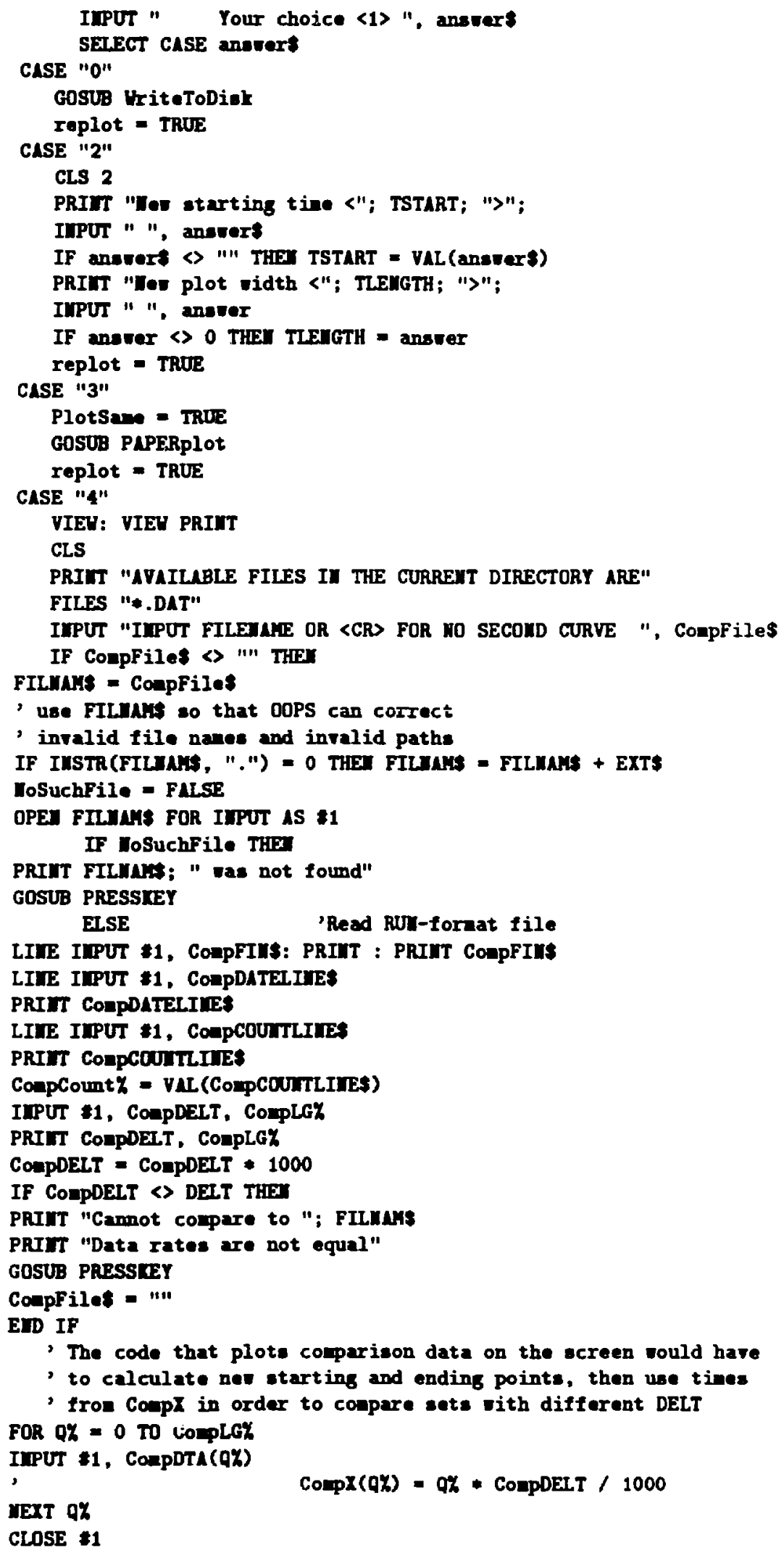


IF CompLGX < LGX THEll 'pad vith zeros

FOR QY = COIPLGK + 1 TO LOK

ConpDT $L(Q \%)=0$

TETT QX

EID IF

EVD IF

EDD IF

CLS

replot = TRUE

GASE ELSE

- leare the loop and go to the main nenu

roplot - FALSE

END SELECT

LOOP UHILE replot

VIEU ' reeet entire screen to graphice vierport so CLS vill clear it

VIII PRIII, also set entire ecreen to text vietport

1950 RETURI

PAPERplot:

1960 REy This is the plot routine.

1970 VS = "tistets ittits"

1980 CLS 2

1990 OPE "COH1:9600,5,7,1,RS,CS55535,DS,CD" FOR RAMDOH AS $\div 1$

2000 XLABS = "TIHE [nicrosec]"

2010 XLABS = ILAB + " " + STARTDATE + " + + STARTTINES

2020 YLABS = "LVE IIT [DV] for" + STRs(Countz) + " sHotS"

2030 PRITT

2050 a $=22.5 / .025$

$2060 \mathrm{EI}=256.5 / .025$

$2070 \mathrm{SY}=16.5 / .025$

$2080-182 / .025$

2090 DER = EI - AX: DEY = ey - SY

2100 IF DEI > DEY THE CH $=$ DEX / 60 ELSE CH $=$ DEY / 60

$2110 \mathrm{CU}=2 * \mathrm{CH} / 3$

2150 PRIIT "The axis labels rill be printed nor-MUTE SURE PLOTIER IS READY--."

2160 PRIIT " Prese any key when ready--."

2170 As - ILETS: IF As = "" THEI 2170

TITLES = FIIIS

PRIIT *1, "III; SP1; PA"; EX; or; ";"

PRIIT *1. "CP"; -LEIT(TITLE\$) - 2;" .5; LB"; TITLES; CHR\$(3)

TITLSS = FILUNA

PRIIT *1, "PA"; EX; ey; ";"

PRIIT *1, "CP"; -LEI(TITLE\$) - 2; " -1; LB"; TITLES: CHR\$ (3)

PRTIT 1 , "SI";

PRIIT \$1. USIMG V\$; CH $* .0025 ; \mathrm{CH} * .0025$;

PRIIT *1, ";"

$2180 \mathrm{LTK}=$ LEN(XLABS)

2190 LWT - LET (YLABS)

2200 PRIIT 11 . "PL";

2210 PRIIT E1, USIMG V\$; $\Delta x$; SY;

2220 PRIIT 1 , "PD";

2230 PRIIT 11, USIMG V\$; sx; ey; EX; ey; EX; SY; sx; SY;

2240 PRIIT 11, "PU;"

2250 ILAB $-(D E X-I I X * C H) / 2+8 x$

2260 YLAB $=$ (DET - ILY * CU) $/ 2+S Y$

2270 PRIIT 11 , "PL";

2280 PRIIT 11 , USIIG V\$; ILAB; SY $-3.5 * \mathrm{CH}$; 


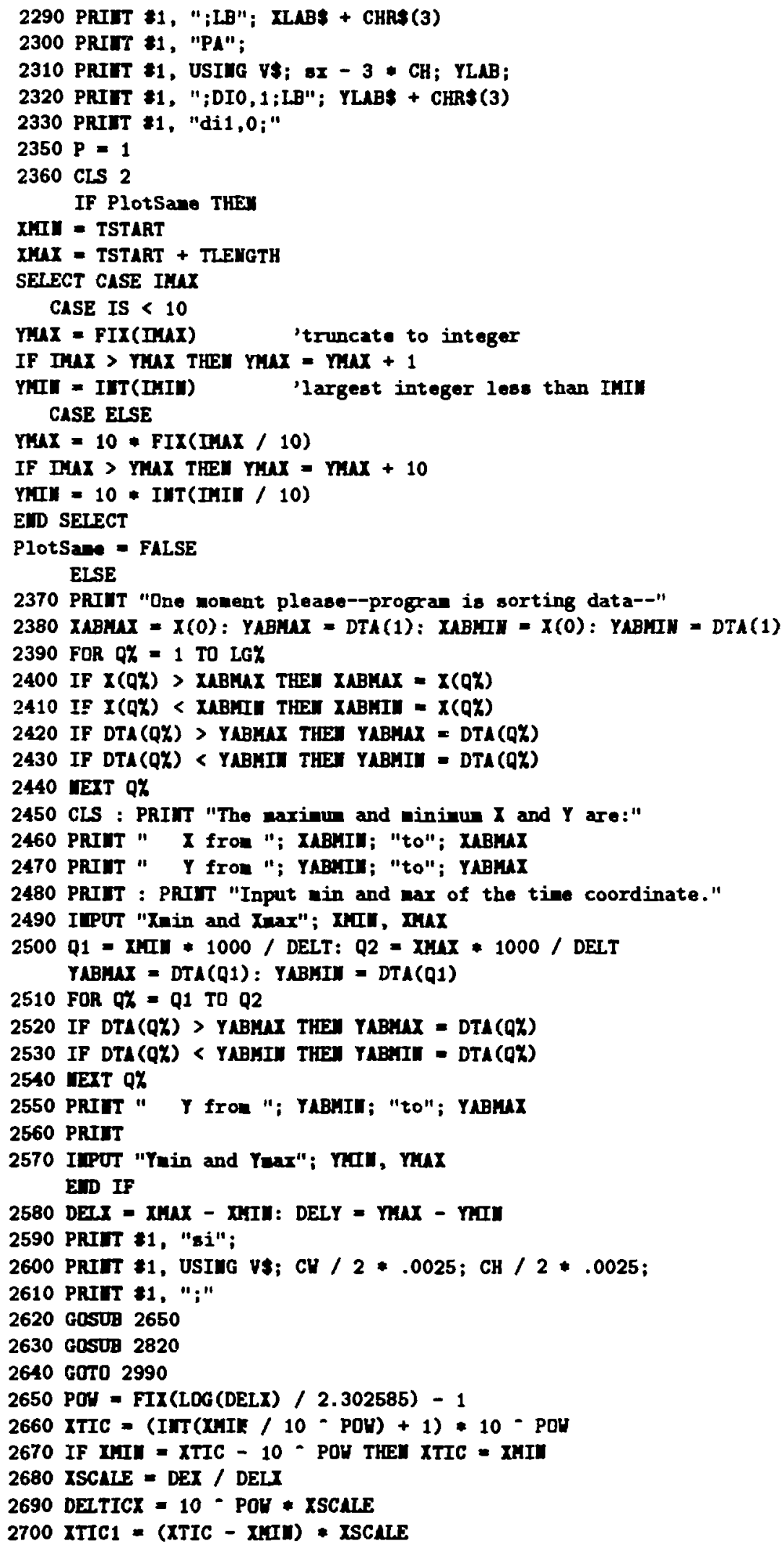




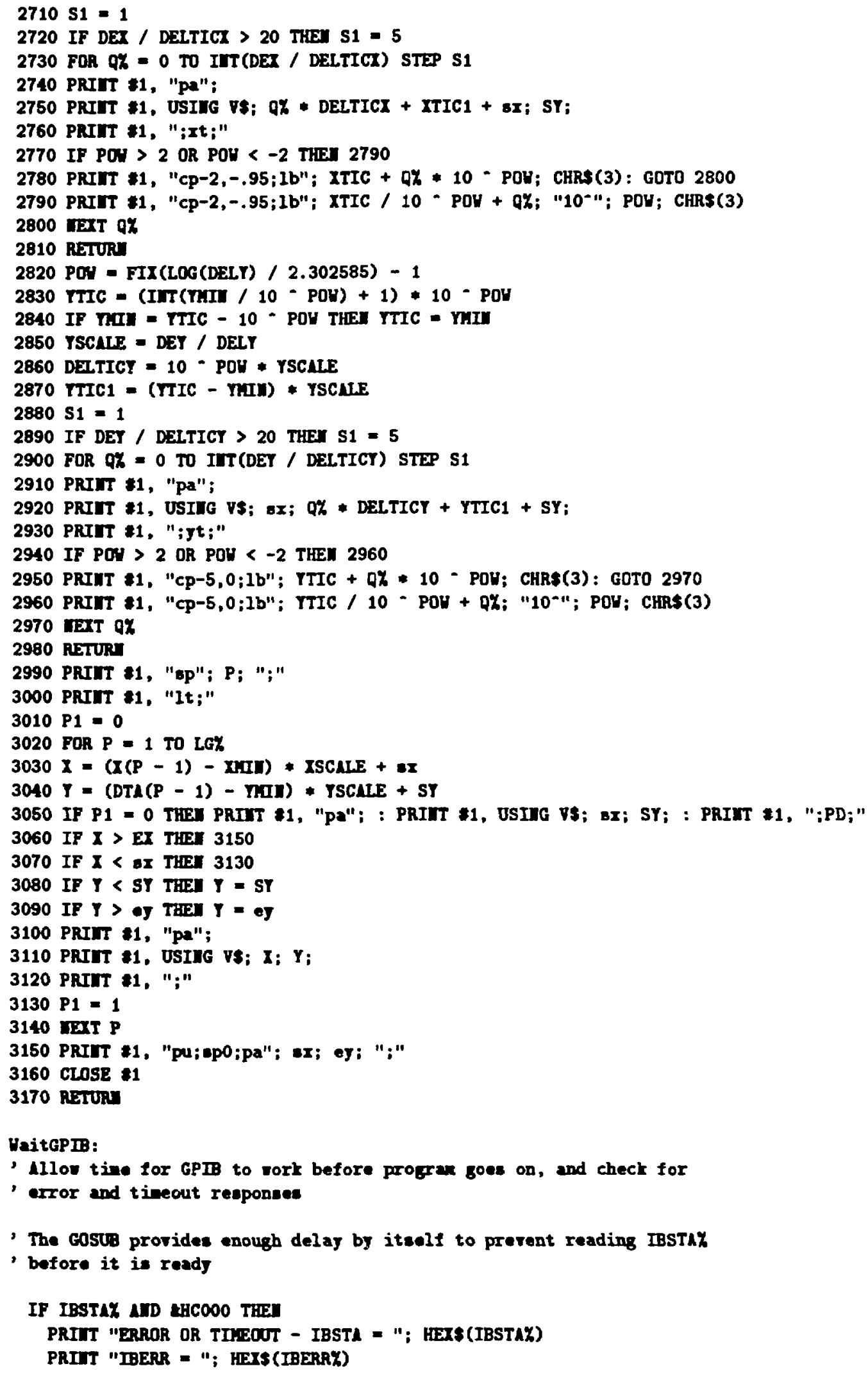




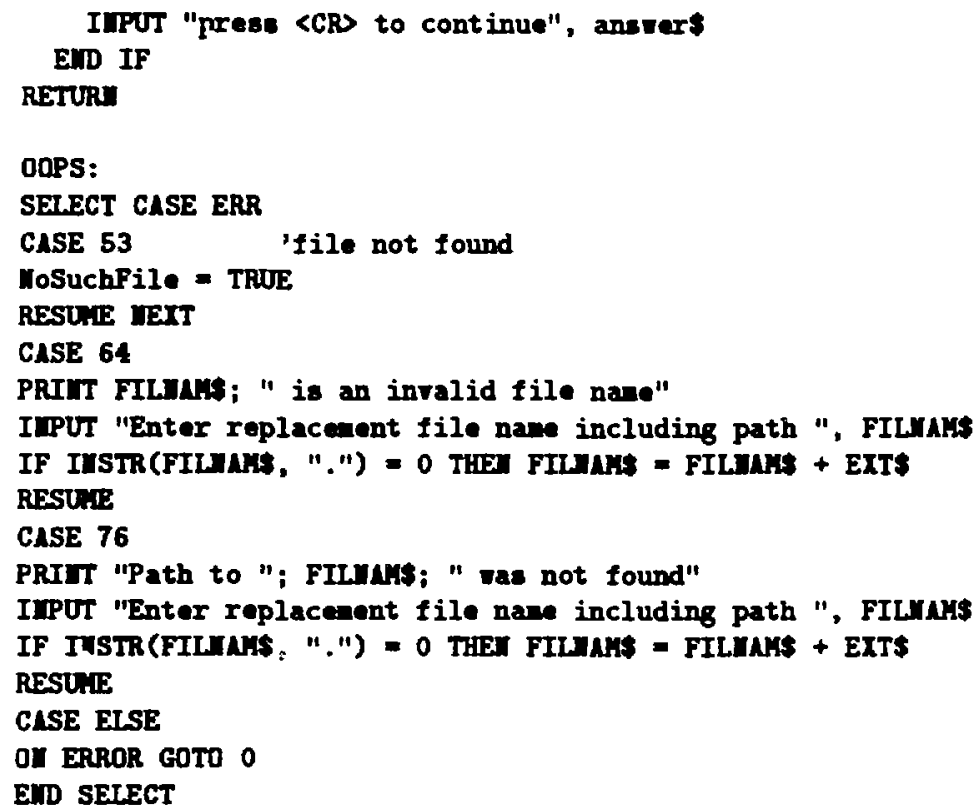

\section{CONVERT1}

This assembly language subroutine is functionally identical to CONVERT, but is $50 \%$ faster. It unpacks TR8818 output values that are initially stored two to a computer word and converts them to BASIC integer format.

coment 1

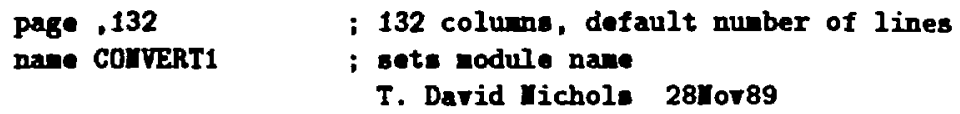

Subroutine to convert digitizer data array to BASIC integer array

Coprright, 1990, The Regents of the University of California. This softera vas produced under a U.S. Governent contract (U-7405-EIG-36) by Los Alanos Iational Laboratory, which is operated by the Univereity of California for the U.S.

Departnent of Energs. The U.S. Goverment is Iicensed to use, reproduce, and distribute this softrare. Pernission is granted to the public to copy and use this software vithout charge, providad that this Motice and any statenent of authorship are reproduced on all copies. Neither the Government nor the University wakes any varranty, exprese or inplied, or assunes any liability or responsibility for the use of this software.

This does the sane vork as the COIVERT subroutine used vith QuickBasic 3.0 in TR88QP7 and related prograns. Both arrays nust be located in DGROUP. Vithin the QB4.5 environnent, this rGquires they be first dinensioned $v$ ith conetant disensions, then assigned to COFAOI. In QB4.5 EXE files, they need not be in corison. 


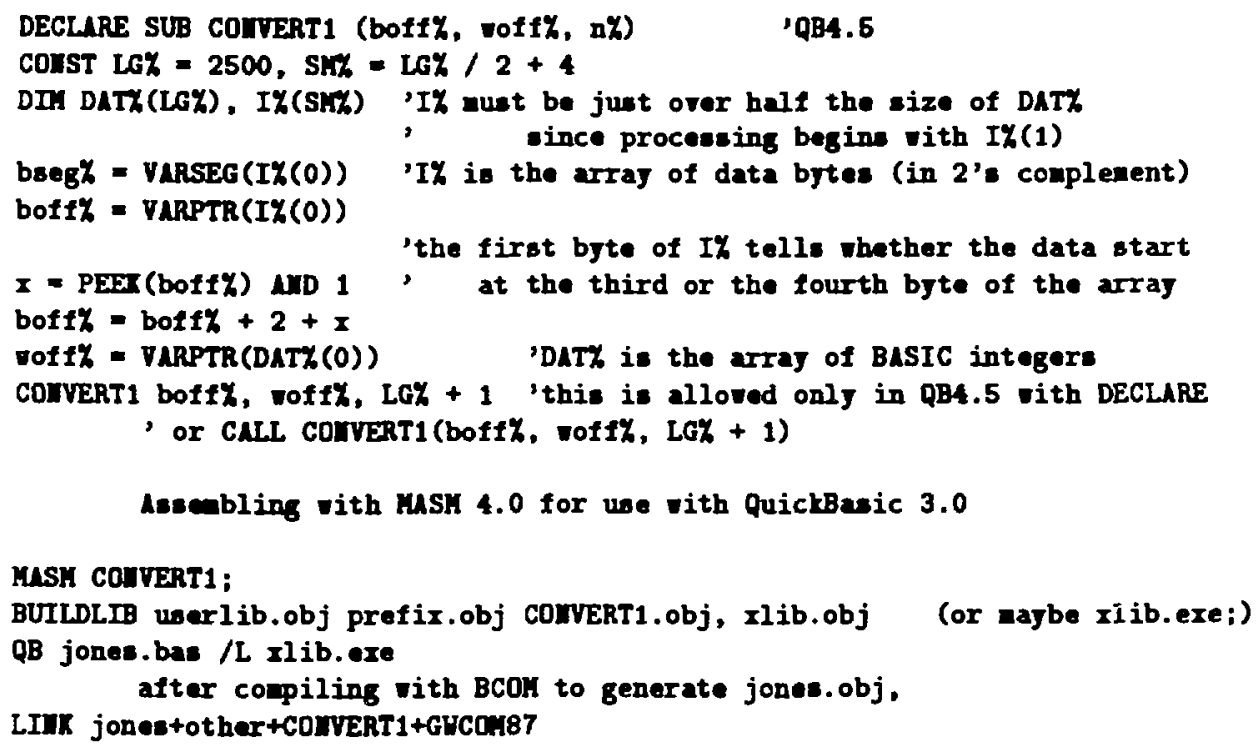

This creates a Quick Library and a stand-alone library, each containing object code of converi, other, and whatever. Object files an be added to or deleted fron these files bJ LIB and by QB itselt (inetructions in Learning and Using rolue of the QB4.5 nanual). ".obj" naj be onitted. File nases in lover case represent actual nanes of files. The two library file nust hare the sane base nave.

; print this title on each page of printout title COUVERT1

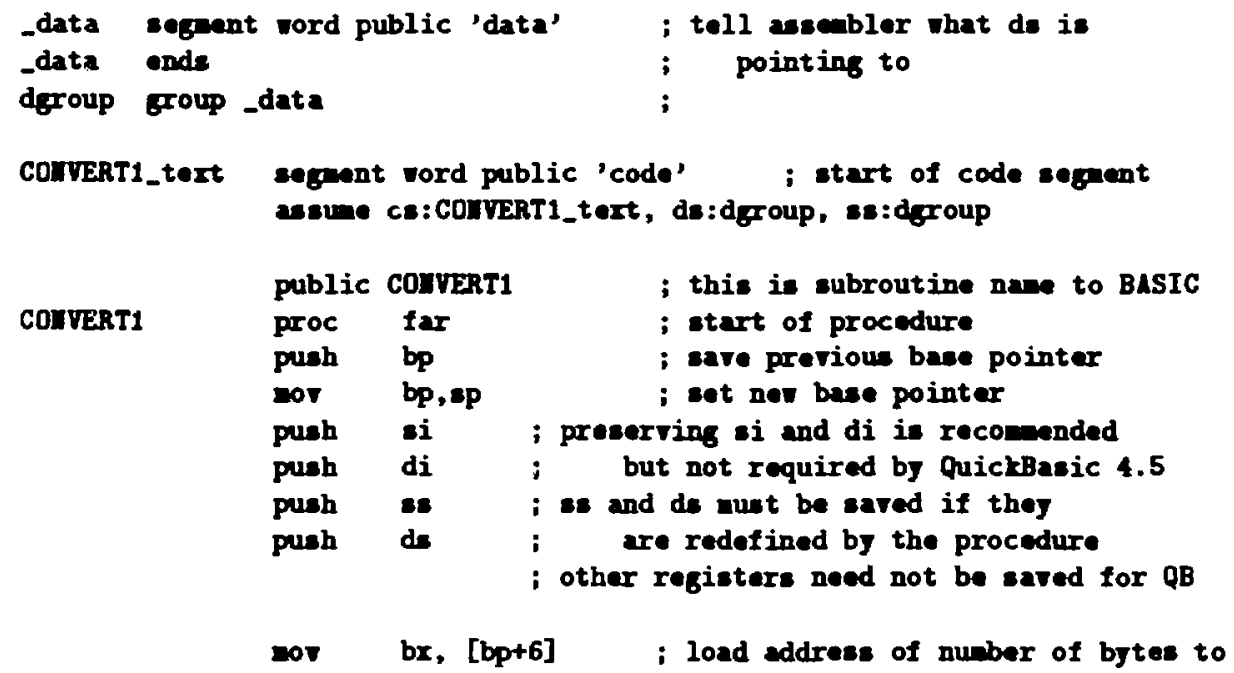




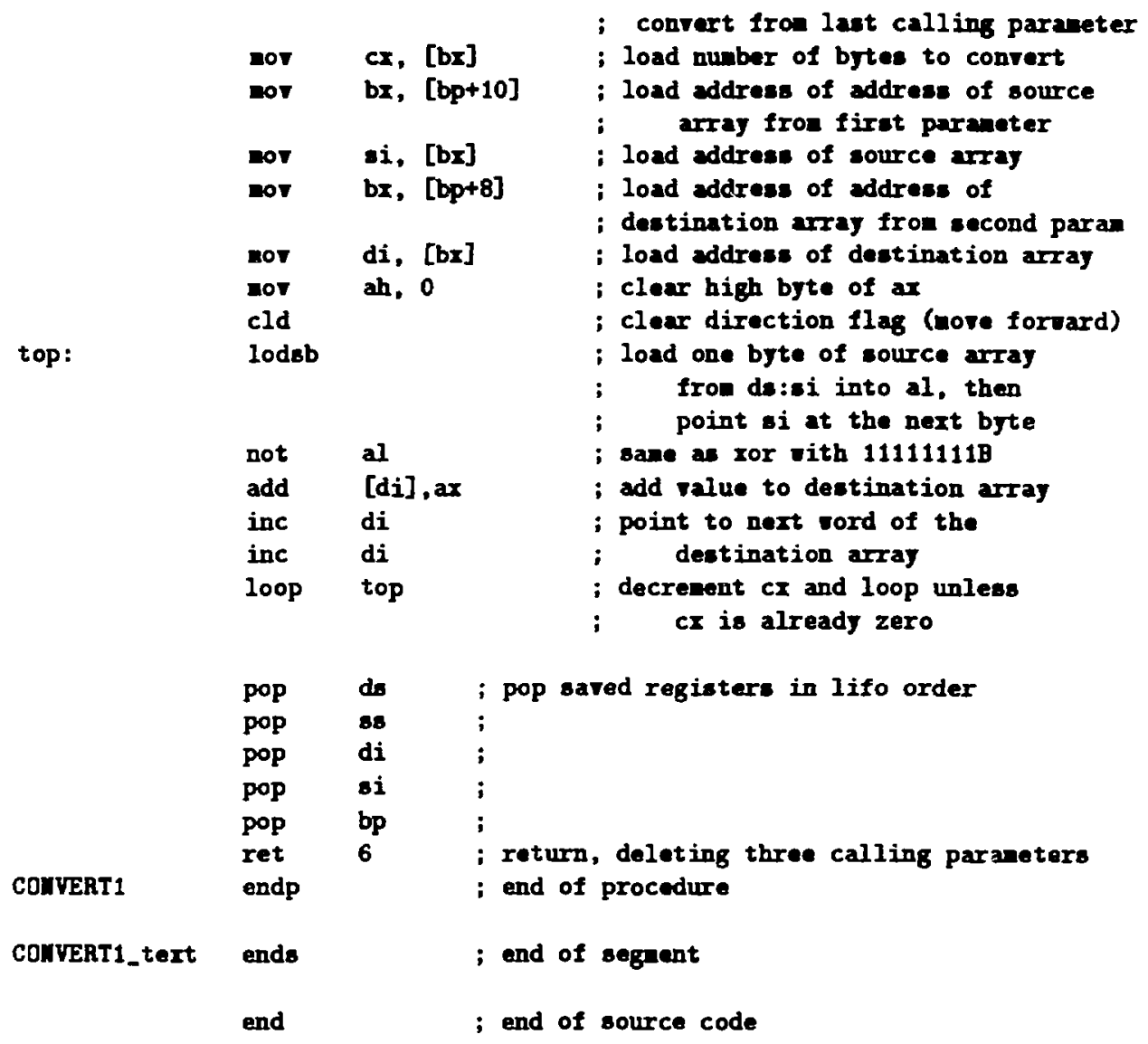

\section{ERUN}

This program reads pulse energies from the joulemeter and averages the spectra by energy bins. It displays the bin number assigned to each new spectrum to make it easier to notice a drift in average pulse energy. It writes a single output file containing averages from all bins with data, and optionally writes data from a single bin in RUN format.

\section{ERUI. BAS 28Sep89}

\footnotetext{
Read Th8818 digitizer, read Gentec, assign each data set to a bin

according to its om laser pulse energy

Based upon ERUT2249 and

1 rea $\ldots \ldots \ldots \ldots \ldots \ldots \ldots \ldots \ldots \ldots \ldots \ldots$ [tr88qpg1. ban] $\ldots \ldots \ldots \ldots \ldots \ldots \ldots \ldots \ldots \ldots$.

2 ren .. nodified tersion of tr88qp7.bas $\quad$-B1

3 ren .. changed line 1150 to subtract zerosig rather than add it $\cdots g^{1}$

4 ren ...

5 ren .. conpile and link []+qbib+convert $\quad \cdots g^{1}$

, also +GUCOH87 if compiled vith BCOH
} 


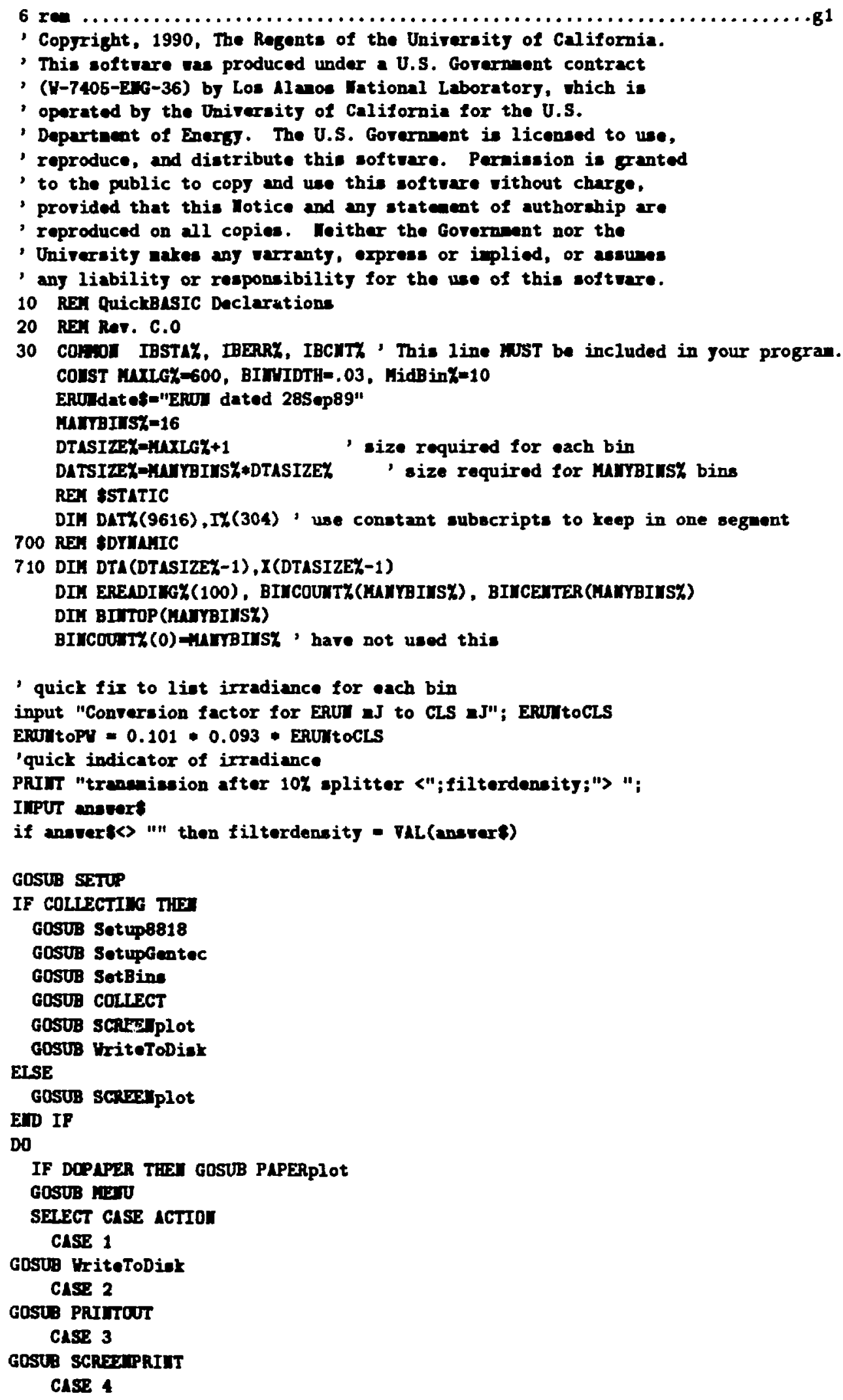




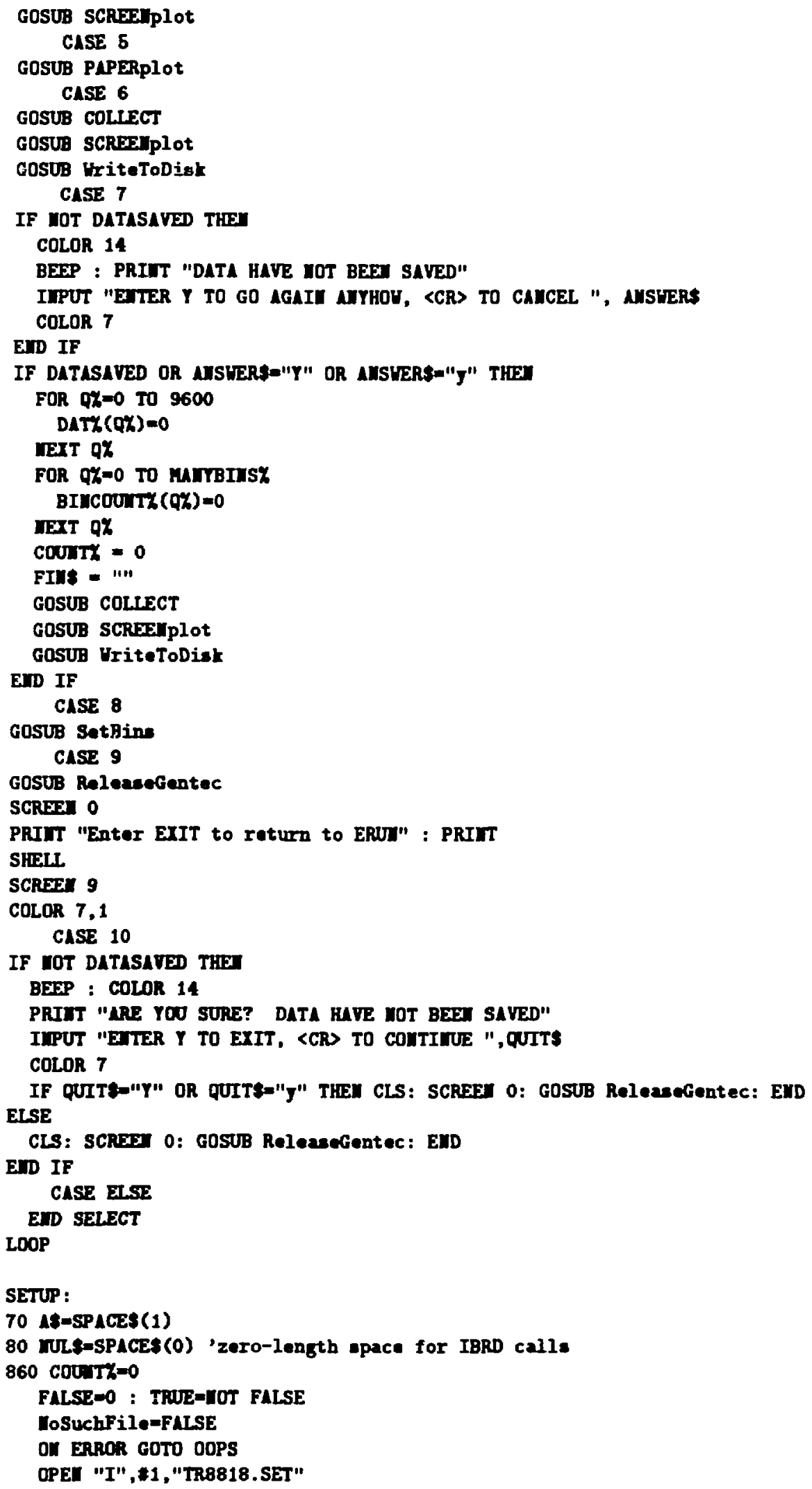




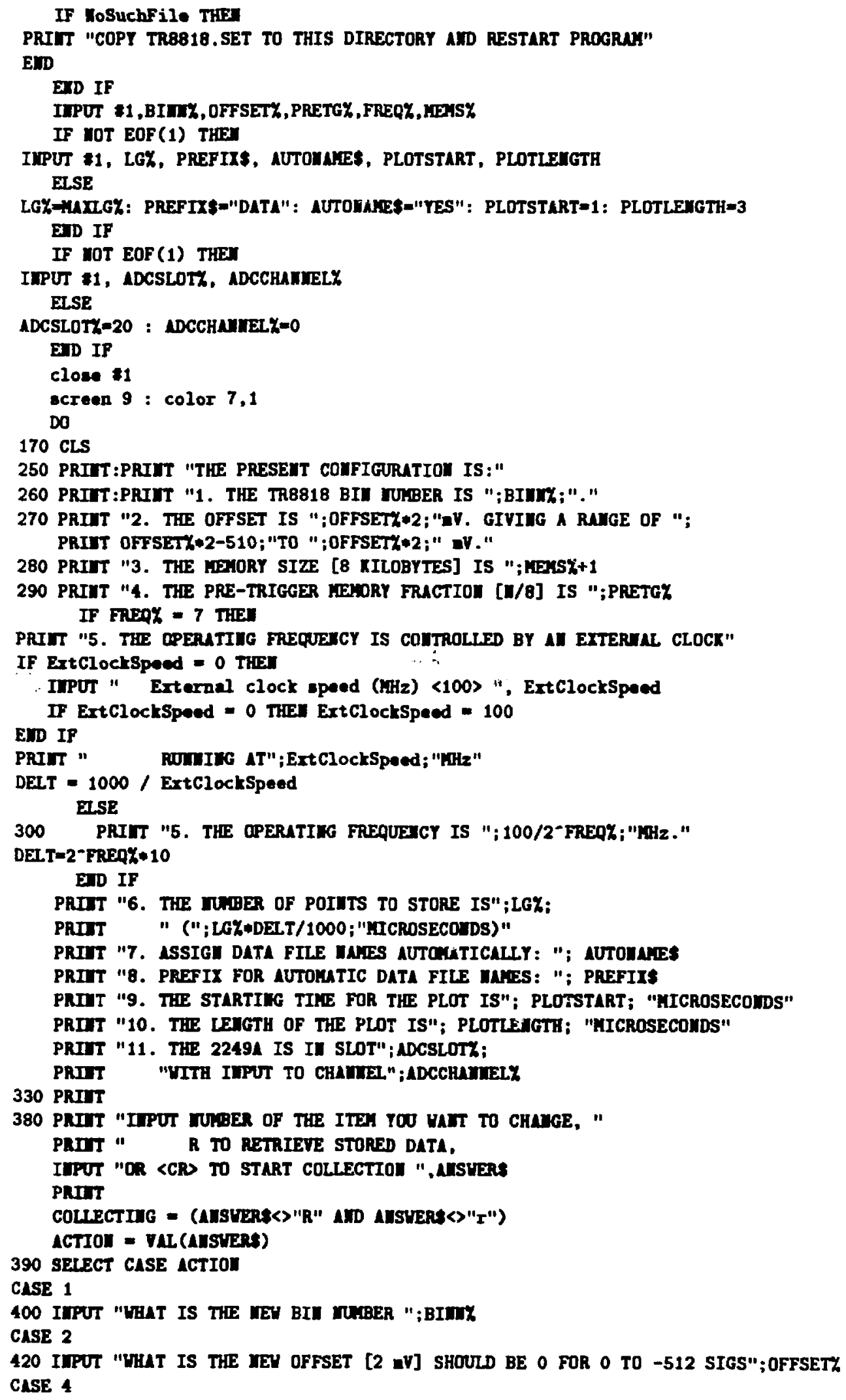


440 IIPUT "WHAT IS THE WEV PRETRIGGER FRACTION [1/8 REYI]";PRETGX

CASE 5

460 PRITT "EMTER FREQUEICY F II THE FORM 100/2^F [MHz] "

PRIIT "O for $100 \mathrm{HHz}, 2$ for 50HHz, etc. or 7 for external clock"

IMPUI "YOUR CHOICE"; FREQK

IF FREQZ $<0$ OR FREQZ $>7$ THEN COTO 460

IF FREOY $=7$ THEN

IIPUT "External clock speed (MHz) <100> ", ExtclockSpeed

IF ExtClockSpeed = 0 THEI ExtClockSpeed $=100$

DEIT $=1000 /$ ExtClockSpeed

ELSE

DEL.T $=2-F R E Q \% * 10$

EID IF

CASE 3

480 IUPUT "EMTER MEHORY SIZE [8 KBYTES] "; KEEHS\%

490 HEIST $\Rightarrow$ IFIST-1

CASE 6

IUPUT "ENTER IURBER OF MICROSECOUDS TO STORE ", AUSUER

AMSUER = AMSTER * 1000/DELT

IF ANSWER C-HLTLGX THEN

LGX $=$ AISUER

ELSE

PRIIT "NOT EMOUGH SPACE FOR"; AMSWER;"KICROSECOMDS"

BEEP : LGX-MUXLGX

EIID IF

CASE 7

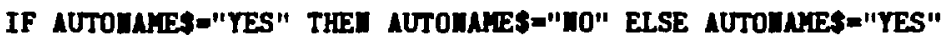

CASE 8

IUPUT "EMTER PREFIX FOR DATA OUTPUT FILE: ",PREFIX\$

CASE 9

IUPUT "EITER STARTIMG TIEE FOR PLOTS: ", PLOTSTART

CASE 10

IUPUT "ENTER LEIGTH OF PLOTS: ", PLOTLEMGTH

CASE 11

IUPUT "EITER 2249A SLOT ITRBER: ", ADCSLOTK

IJPUI "ENTER 2249A CHUHLEL TURER: ", ADCCHANTELX

CASE ELSE

EDD SELECT

LOOP MHILE ACTION

IF COLIECTIMG THED

OPEI "O", $\$ 1, "$ TR8818.SET"

URITE ะ1, BIIIY, OFFSET\%, PRETCZ, FREQZ, FEISZ

URITE $ะ 1$, LGX, PREFIXS, AUTOMAMES, PLOTSTART, PLOTLENGTH

VIITE *1, ADCSLOTh, ADCCHANTEIX

CLOSE 1

ZEROSIG $=0$ FFSETH $* 2$

ELSE

COSUB Readfrominak

EUD IF

AUTOWARE - (AUTOMARES="YES")

FOR $Q X=0$ TO LGX

$x(Q X)=Q Z * D E L T / 1000$

IEIT QH

RETURT

Setup8818: 


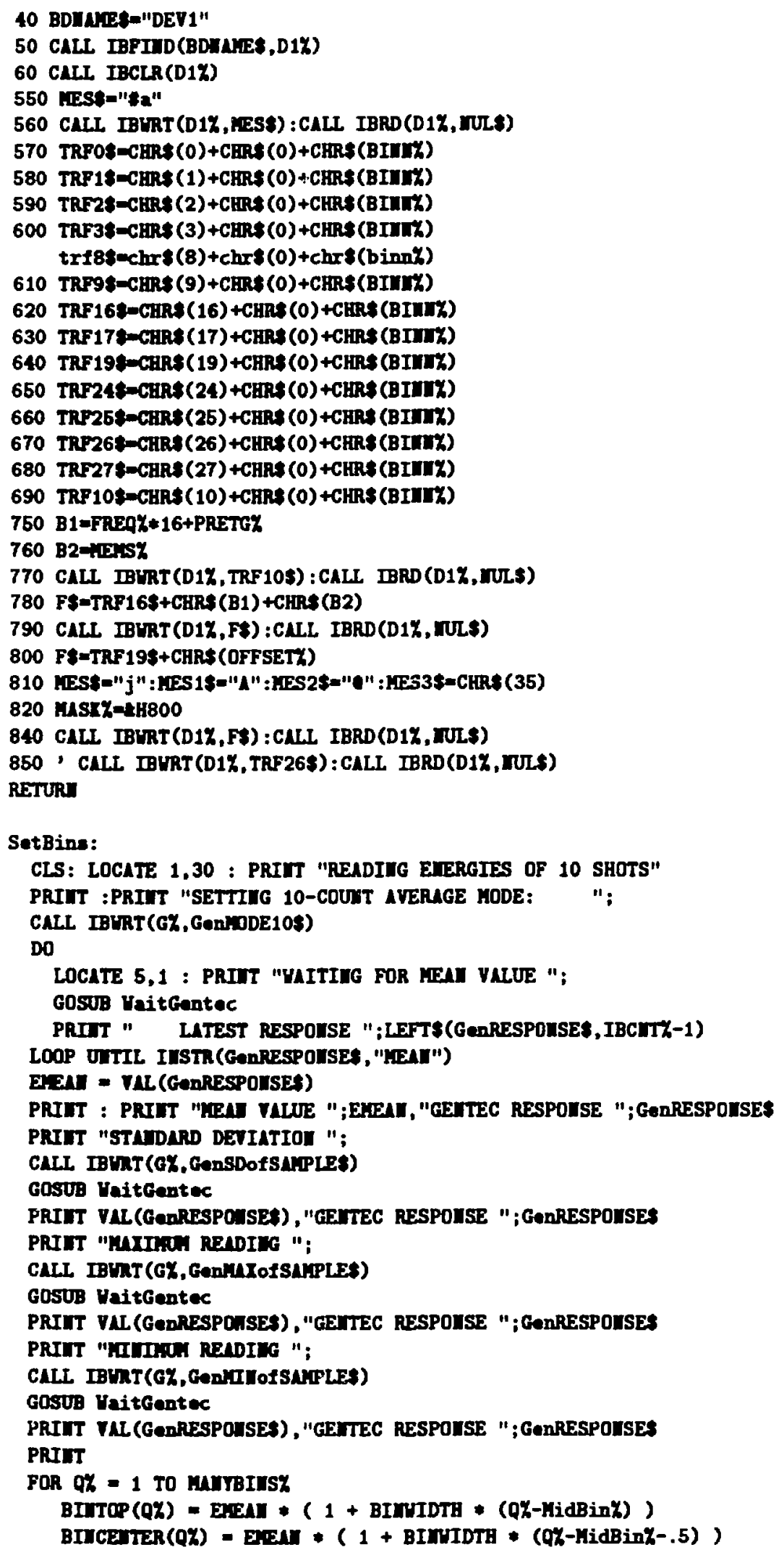




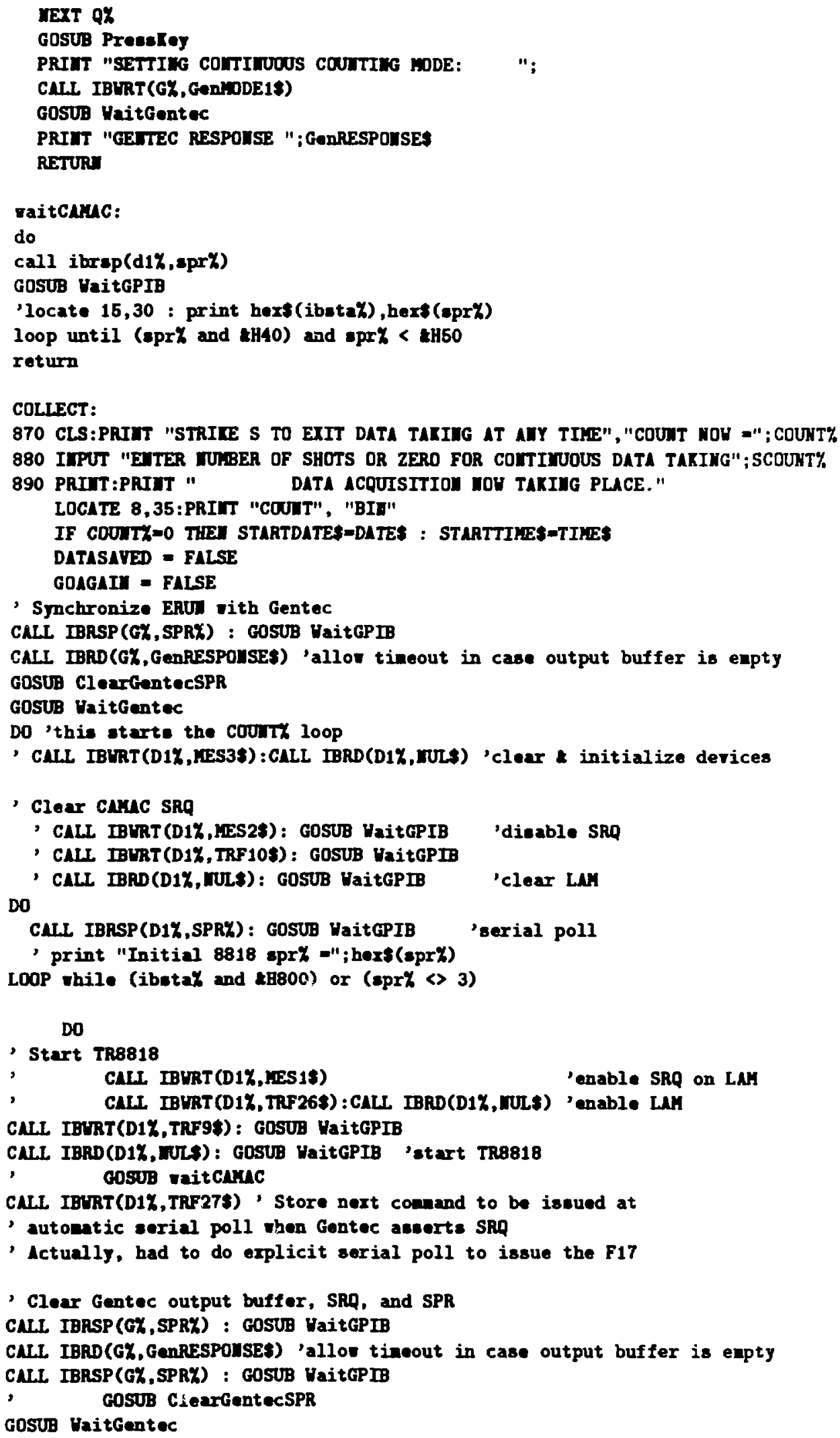




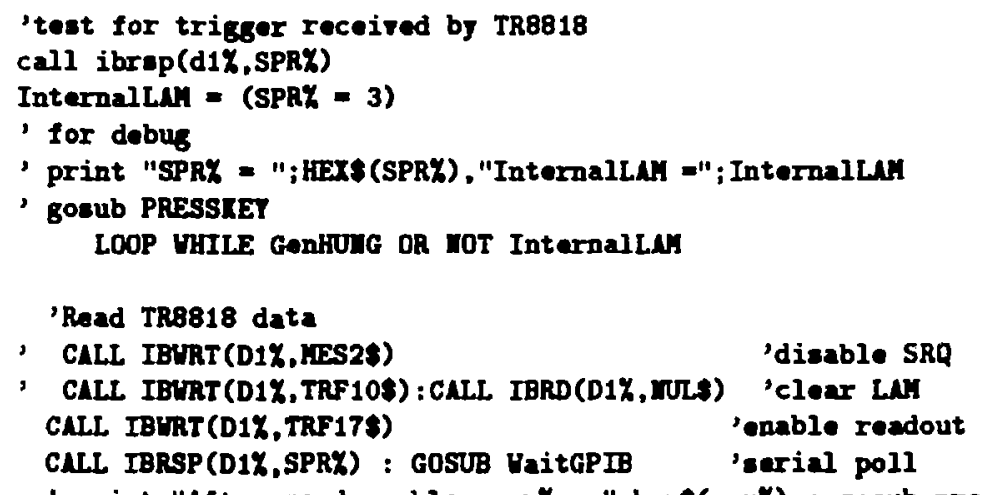

'print "After read enable, spr\% = "; hers(spr\%) : gosub presskey

CALL IBLRT(D1X,NES\$): COSUB VaitGPIB 'high speed 16 bit block read CALI IBIRT(D1\%,TRF2\$): GOSUB HaitGPIB

CALL IBRDI(D1X,IX(0), LGK+4): GOSUB HaitGPIB 'read data to IK array

, CALI IBRSP(D1X,SPRY) : GOSUB UaitGPIB 'sorial poll

' print "After block read, spr\% = "; her\$(spr $\%)$ : gosub presskey EMERGY = VAL (GERRESPOUSE\$)

- find bin number - range is 1 to MurgIns\%

EBIUK-iUUTBIWS\%

FOR $Q \%=1$ TO MUIYBIMSK - 1

IF EIERGY < BIMTOP (QY) THEN EBIMY $=Q \%$ : EXIT FOR

WEXT QX

- DIVIDE GENERATED DATA BETVIEEN TWO BIMS

, IF COUWTK MDD 1 THEU EBIWK=1

BIWOFF SETY $=($ EBIMY-1) * (DTASIZEY)

BIMCOUITK (EBIMX) $=$ BIWCOUITK (EBIMY) +1

- geTrRate DATA

- FOR $Q Y=10$ TO 50 : IX(QX)=2 : IEXT QY

1050 I $=P E E R(V A R P T A(I X(0)))$ AND 1

1060 ADR $K=Y \triangle R P T R(I \%(0))+2+X$

830 ODRY = VARPTR (DATZ (BIMOFFSETK))

1070 COURTX-COUTH +1

1080 LOCATE 10,36:PRIT COUIT, EBIWY

1090 CLLL COWYERT (LDAY, ODRY, LG\% +1)

LOOP UUTIL (COUITK-SCOUTTK) OR (INKEY\$C>"")

STOPTIRES-TIIE:

GOSUB SelectBin

RETUR:

SelectBin:

CLS

PRIIT FIMS

PRIMT "CURREUT BIN IS"; BIWVIEUY

Do

PRInT

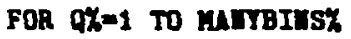

IF BICCOUTY(QK) THEN COLOR 14 ELSE COLOR 3

'PRIIT , "BII"; Q\%, BIMCOUMT\%(Q\%) ; "COUITS" 


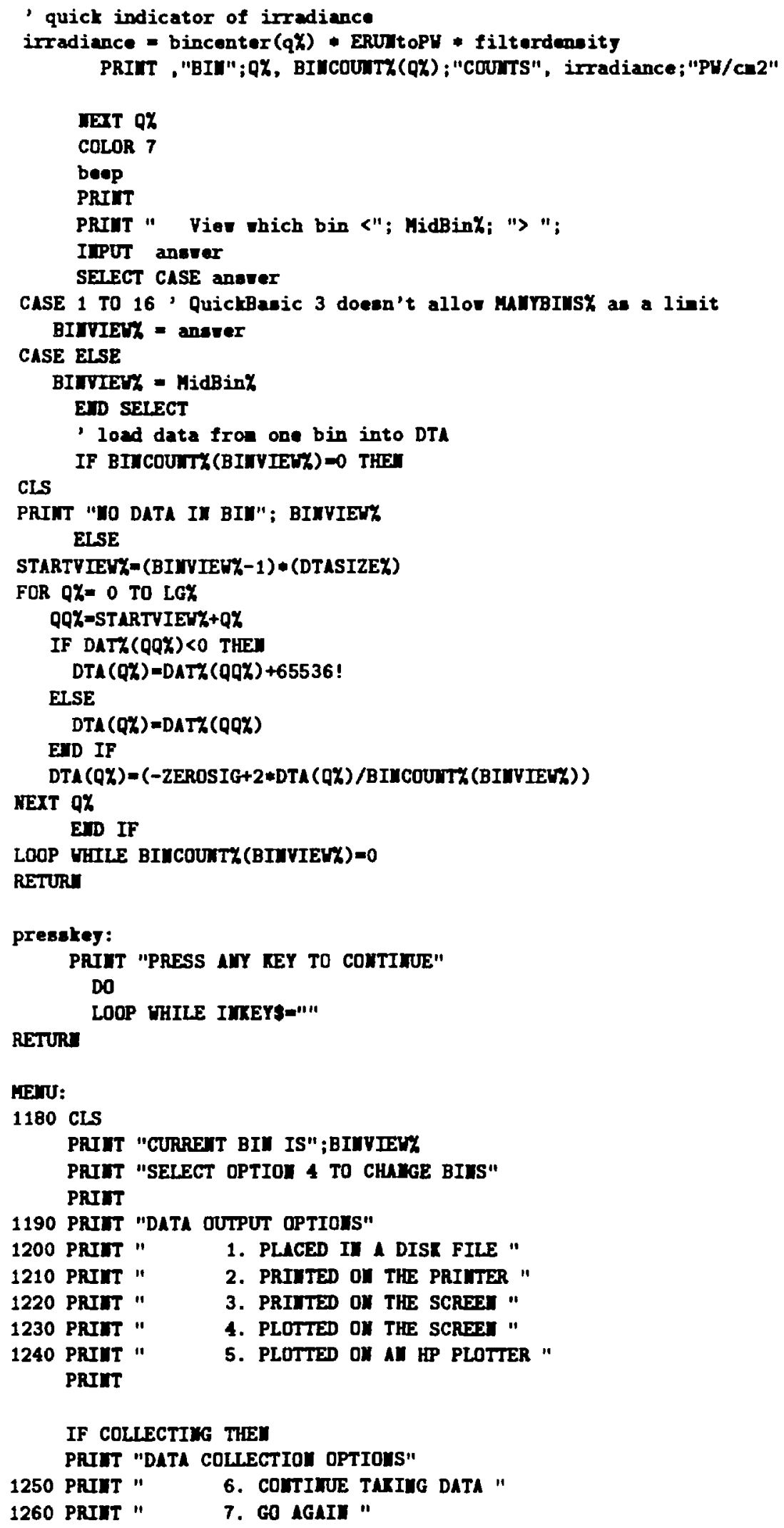




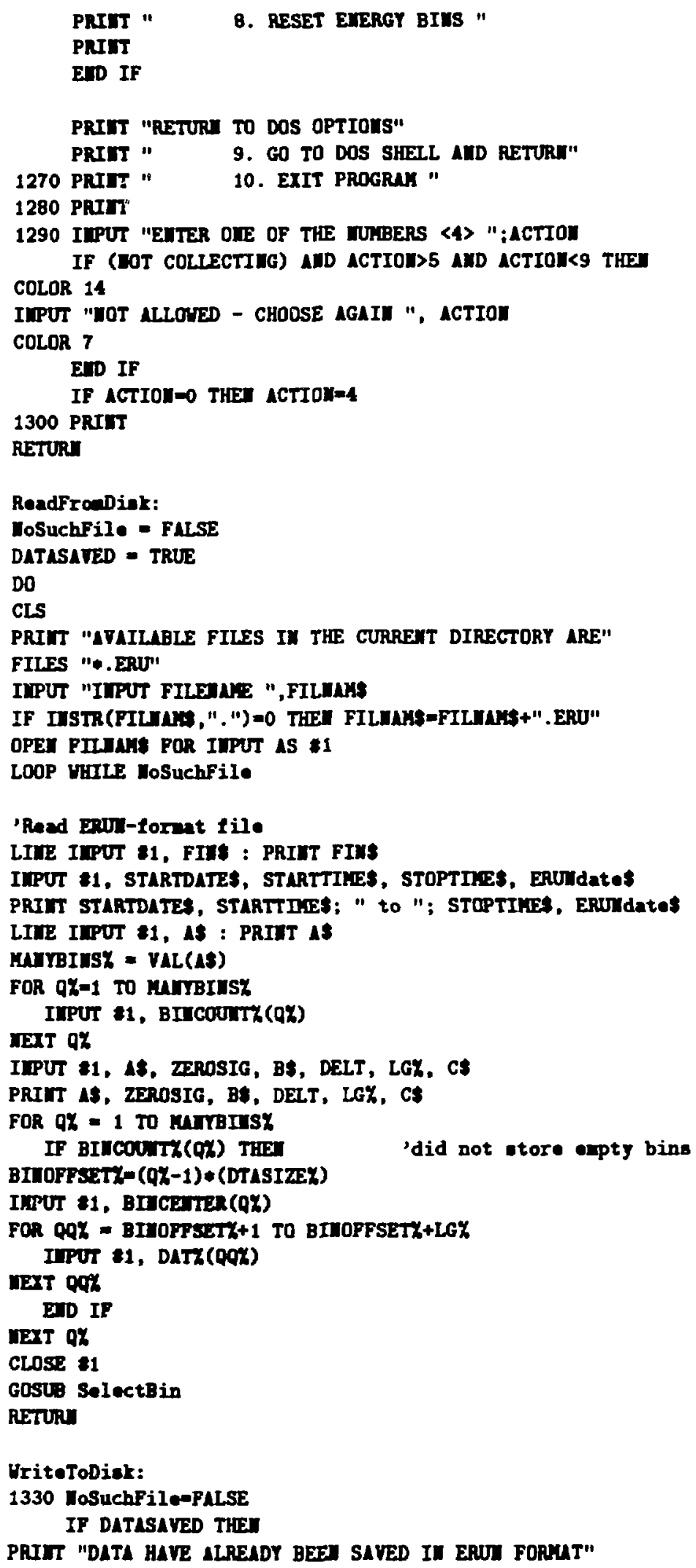


PRIIT "READY TO URITE FROA BIT";BIWVIEUX;"I" RUD FORHT" : PRINT IUPUT "EITER AIT CHARACTER TO VRITE FILE OR CCR> TO CAICEL ", AHSWERS IF AISLERF="" THE FIIS="" ELSE

CLS

IF DOPAPER THEN PRIIT "Paper plot vill be dram" : PRIIT

COLOR 14

PRIIT "READT TO SAVE DATA AS A DIST FILE"

COLOR 7

IIPUT "IIPUT FILE LABEL OR <CR TO CAMCEL ";FIMS

EID IF IF FIMS="" THEU RETUR

DO

IF NUTONAY THF

SEQUEUGE $=$ SEQUENCE +1

SEQF-STRE (SEQUEXE)

LSEQ=LEX (SEQS)

If 3-LSEQ>0 THE

SUFFIX \$-STRIIGS (3-LSEQ, "0") +RIGHT\$ (SEQS , LSEQ-1)

EISE

SUFFIX:-RIGHT\& (SEQ\$, LSEQ-1)

EID IF

FIJAIAH=PREFIX $\$+$ SUFFIX\$+".ERU"

PRIIT : PRITT "FILE MARE UILL BE ";FILMAHS : PRIUT

EISE

IUPUT "IIPUT FILETARE ",FILUAKs

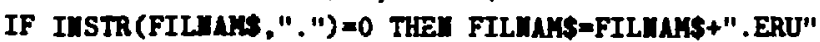

EID IF

OPEI FILWAM FOR IIFUT AS 1

IF MOT HoSuchFile THE

CLOSE $\$ 1$

PRIIT FILUNAF; " ALRELDT EXISTS. ";

EID IF

LDOP UITIL HoSuchFile

OPEI FII INA FOR OUTPUT AS $\$ 1$

IF DATASAYED THEH

1350 PMIIT $\$ 1$, FIM:

1360 PRIIT *1,STARTDLTE\$;", ";STARTTIHE\$;" to ";STOPTIHEs;

PRITT \&1," BII"; BIUYIETK; " CEDTER EIERGY"; BIUCEUTER(BIUVIEUK)

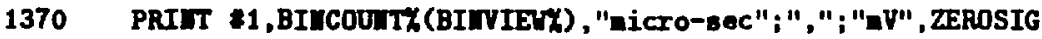

1380 PRIIT \$1,DELT/1000,LGX

1390 FOR QK $=0$ TO LGK

1400 URITE $\$ 1, D T \Lambda(0 \%)$

1410 UEXT OK

EISE

PRIIT *1, FIIS

URITE *1, STARTDATE\$, STARTTIIE\$, STOPTIYE\$, ERUIdate\$

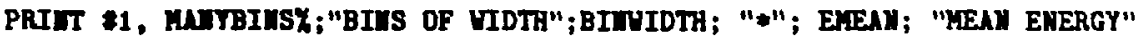

FOR $0 \%=1$ TO HATBIIS\%

PRIII *1, BIICOUIT $(a \%)$;

IEIT QX

URITE 1 ,

WRITE "1, "ZEROSIG" ZEROSIG,"TIMESTEP"; DELT, LG\%; "TIMES IN EACH BIU"

FOR QY $=1$ TO ALTBIISY

IF BImCOUT $(0 \%)$ THEI

BINOFFSETK $-(0 \%-1) *($ TIASIZEK)

'do not store enpty bins

WRITE 1, BIMGDTER(QY)

FOR QQ\% - BIHOFPSET +1 TO BIMOFFSETZ+LGK 


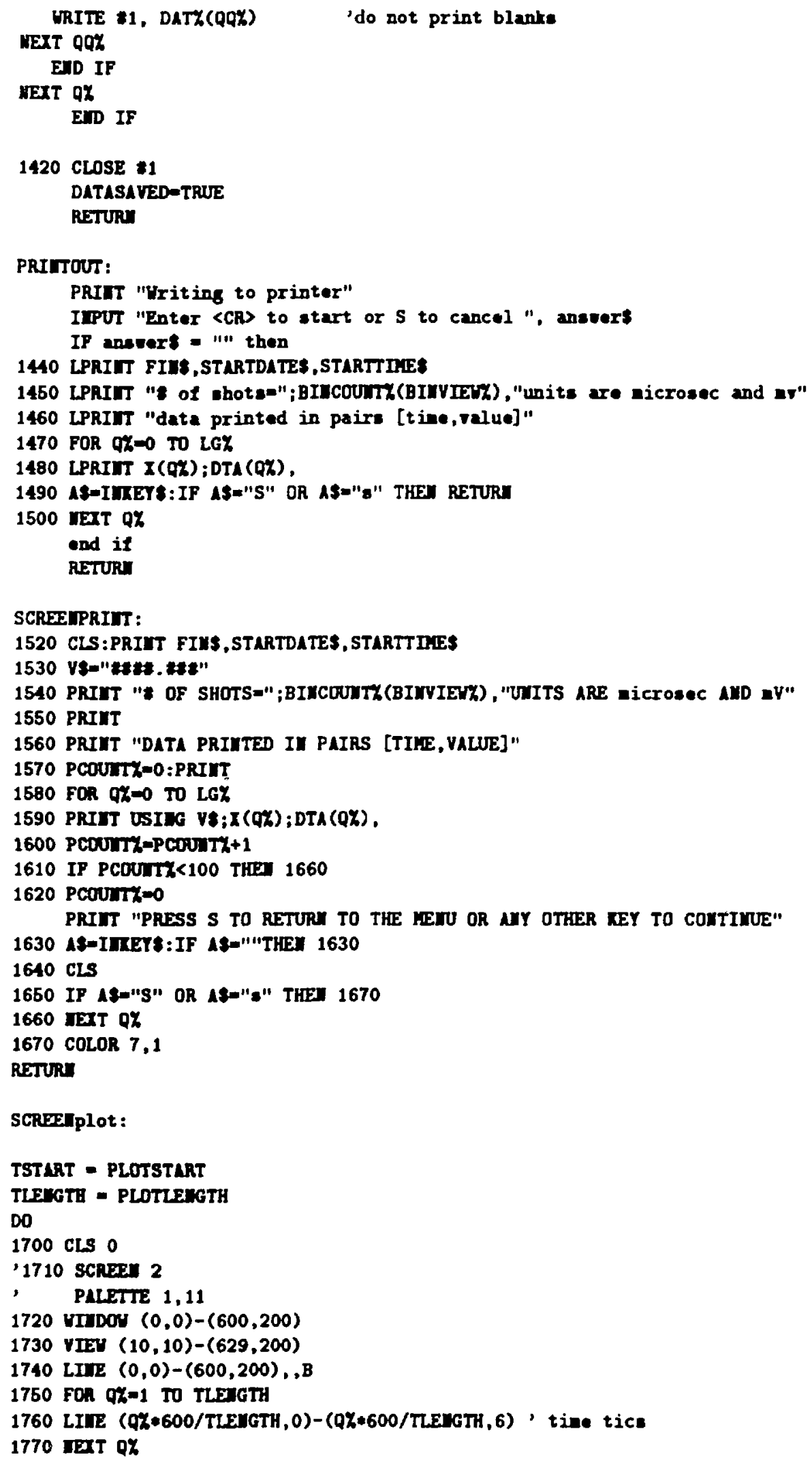




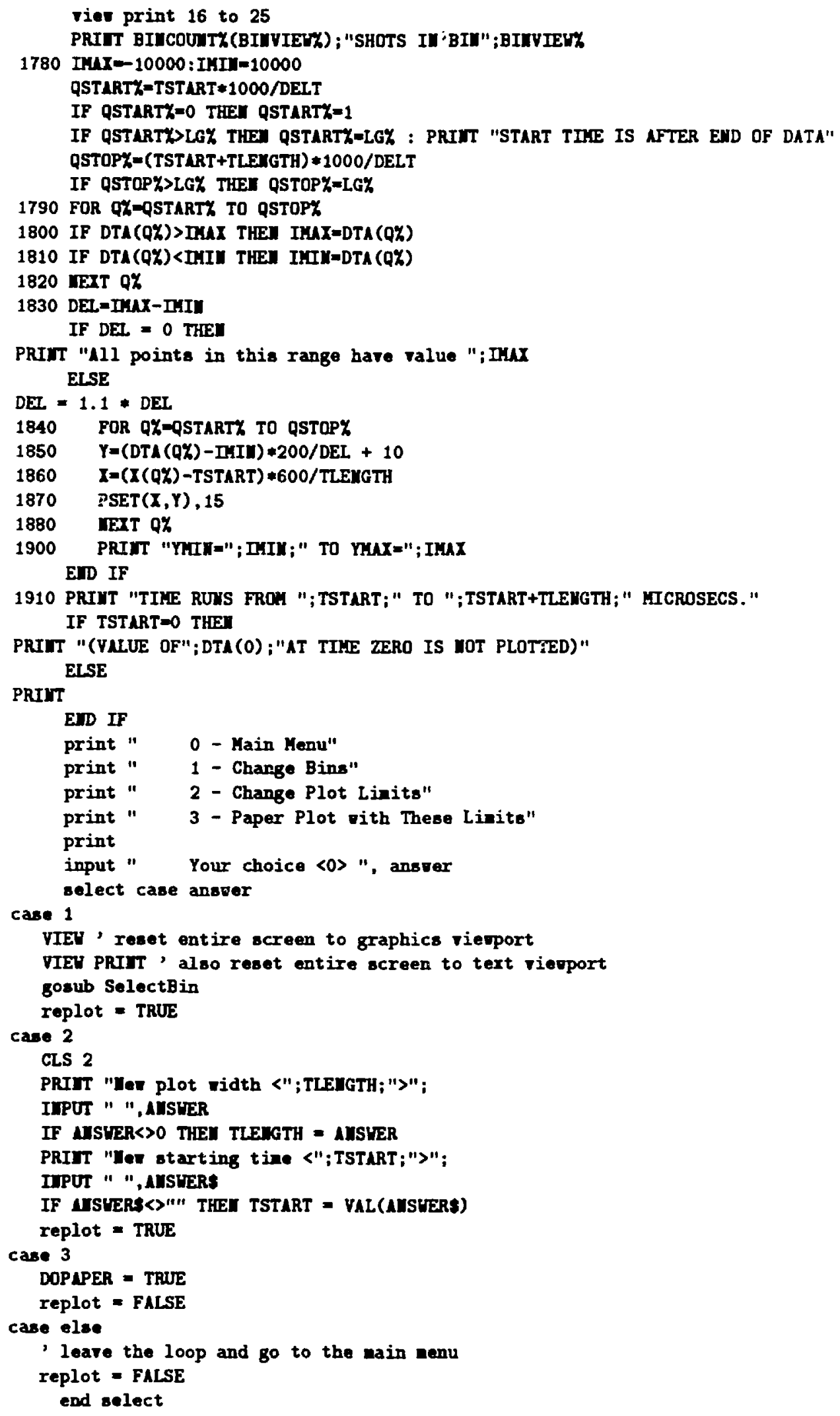




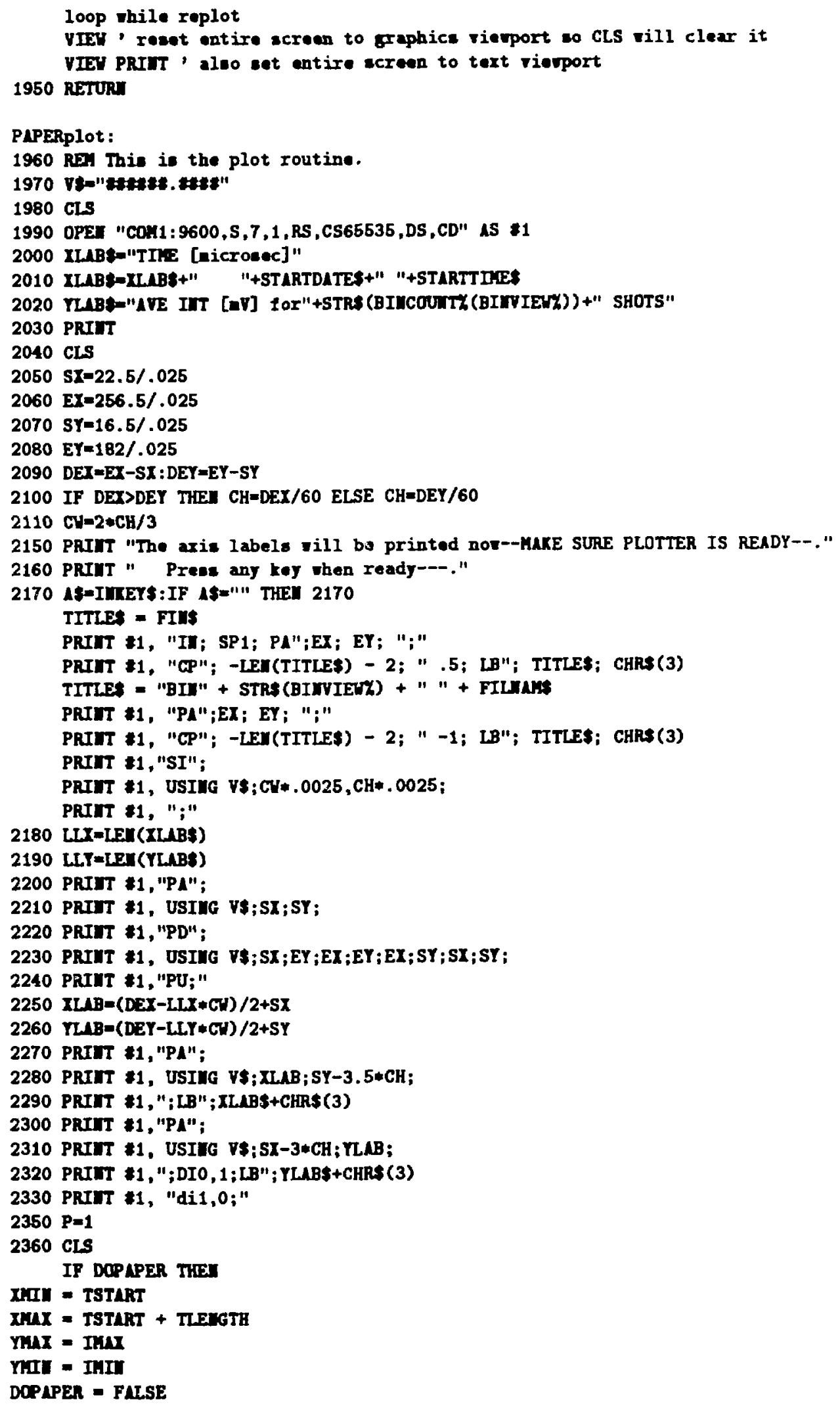


ELSE

2370 PRIIT "One nonent please--prograe is sorting data--"

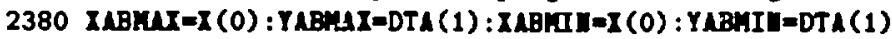

2390 FOR Q $K=1$ TO LGK

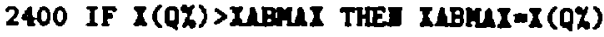

2410 IF $I(Q Z)<T A B M I$ THE $I A B K I I=X(Q \%)$

2420 IF DTA $(Q X)>$ YABMI IHE TABHI-DTA $(Q Z)$

2430 IF DTA $(Q X)<$ YABKI THE YABKII-DTA $(Q X)$

2440 IETT QT

2450 CLS:PRIIT"The maxime and nininu $X$ and $Y$ are:"

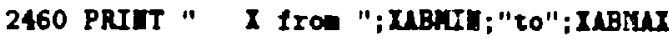

2470 PRIII " Y from "; YABRIU;"to"; Y LBMUX

2480 PRIIIT:PRIIT "Input ain und ax of the tiec coordinate."

2490 IUPU "Znin and Xax"; Xirn, DUX

2500 Q1-IUIE*1000/DEIT:Q2-IKL*1000/DELT

YABMAT-DT $\triangle(01)$ : YABNIIIDTA (Q1)

2510 FOn Qh-Q1 To $Q 2$

2520 IF DTA (QX)> YABMX THEU YABMT-LTA $(Q X)$

2530 IF DT $(Q \%)<Y \wedge B N I$ THEI YABMIII-DTA $(Q \%)$

2540 IETT QY

2550 PRIIT " Y fron "; YABKII:"to"; Y

2560 PRIIT

2570 IIPUT "Yein and Ymax"; MIU, MuX

5ID IF

2580 DEIX =XHAX-XII : DELY - YMUX-YII

2590 PRIJT 1 , " $i "$;

2600 PRIIT $* 1$, USIIG V\$; CH/2*.0025, CH/2*.0025;

2610 PRIIT 1 .":"

2620 GOSUE 2650

2630 GOSUB 2820

2640 GOTO 2990

2650 POUFFIX (LOG (DELX)/2.3025B6)-1

2660 ITIC=(IIT (IMI/10-POU $)+1) * 10^{-P O Y}$

2670 IF IXII-ITIC-10'POU THEM XTIC-XHII

2680 ISCALE-DEX/DELX

2690 DEITICI $=10^{\circ}$ POU *ISCALE

2700 XIIC1=(XTIC-XMII) $* X S C A L E$

2710 S1=1

2720 IF DEX/DEITICI>20 THEX S1-5

$2730 \mathrm{FOn}$ QH 0 TO IIT(DEX/DELIICX) STEP $S 1$

2740 PRIIT 1, "pa";

2750 PRIIT 1 , USIHG V\$; Q $*$ DEITICX+ITIC1+SX,SY;

2760 PRIIT 1 , ";xt;"

2770 IF POY 2 OR POU<-2 THE 2790

2780 PRIII *1, "cp-2,-.95;1b";ITIC+QK*10 -POH;CHR\&(3):GOTO 2800

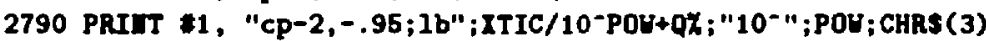

2800 IEXT QX

2810 RETUR

2820 POVFFIX (LOG (DELY) $/ 2.302585)-1$

$2830 \mathrm{TTIC}=\left(\mathrm{IIT}\left(\mathrm{TIII} / 10^{-\mathrm{POH}}\right)+1\right) * 10^{-\mathrm{POU}}$

2840 IF THI=TIC-10-POU THED YTIC=MII

2850 YSCALE $=D E Y / D E L Y$

2860 DELTICY $=10 \%$ POW YSCALE

2870 YTIC1=(YTIC-YMII) *YSCALE

$2880 \mathrm{~S} 1=1$

2890 IF DEY/DELTICY $>20$ THEW $S 1=5$

2900 FOR $Q Z=0$ TO IUT(DEY/DELTICY) STEP S1

2910 PRIIT *1, "pa"; 


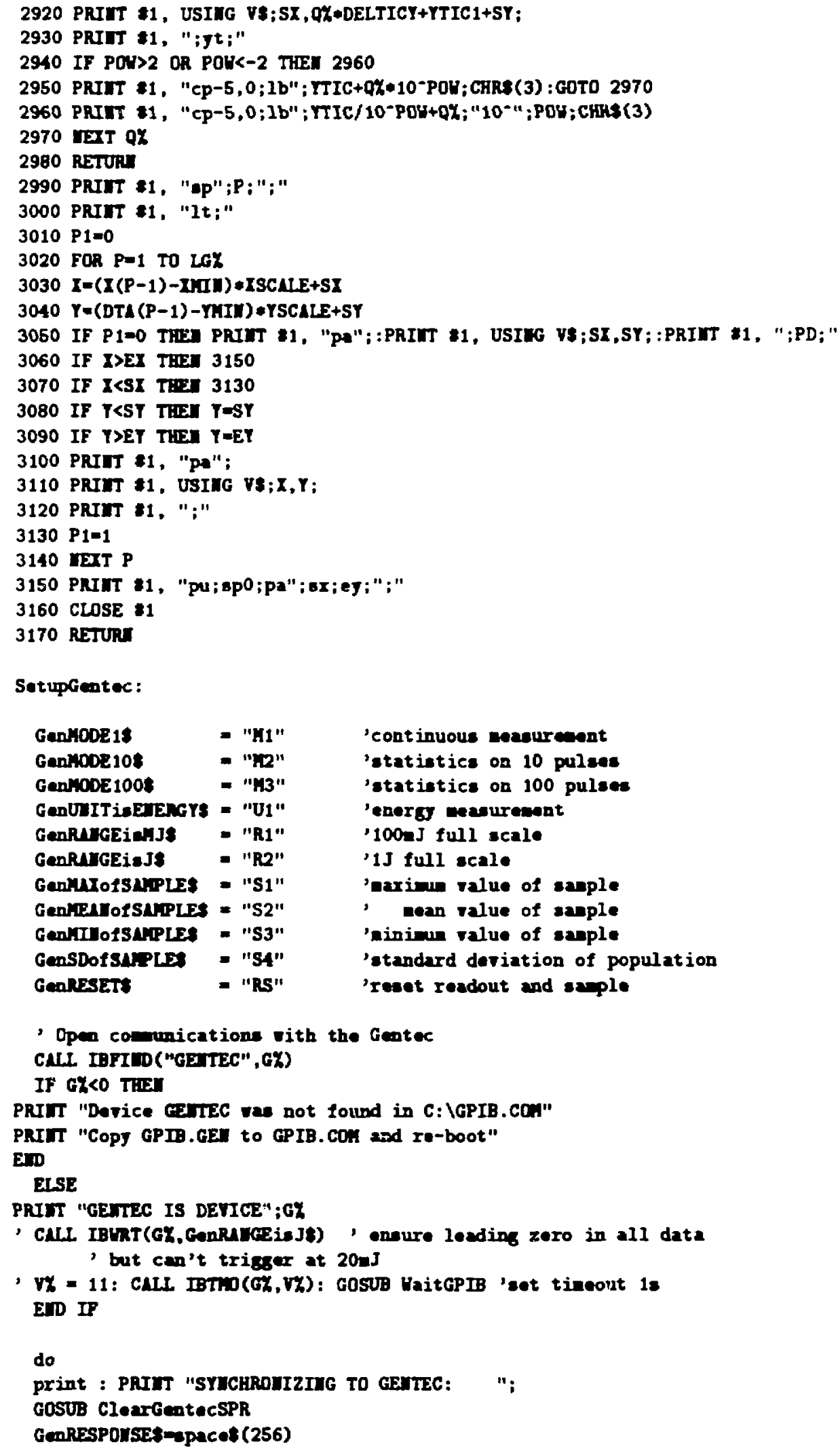


CALL IBRD (G\%,GenRESPOISE) 'allor tinaout in cale output buffer is enpty if ibeta\% and $1 H 4000$ then print "timeout"

CALL IBURT (G\%, GenWODE18)

loop until (ibstaX and th0900) = th0900

gosub VaitGentec

RETURN

HaitGPID:

' Allor tine for GPIB to vork before progran goes on, and check for

' error and timeout responses

' The GOSUB provides enough delay by itself to prevent reading IBSTA\%

' before it is ready

IF IBSTAK AND AHCOOO THEW

PRITT "ERROR OR TIREOUT - IBSTA = ";HEXS (IBSTAY)

PRITT "IBERR - "; HEXS (IBERRY)

PRIT "Is the GEITEC pover on?"

IIPUT "press $\langle C R>$ to continue", answers

EIT IF

RETUR:

WaitGentec:

' Replacesent Ior IBWAIT using aas of tH4800

- Hait for Gentec to request service, notifying user erery tro seconds if

' nothing happens - this usually neans the beas is blocked

' When Gentec requests service, it has something to report, but the nessage

- as be inconplete if the serial poll response byte is nonzero

GOSUB YaitGPIB

GenHUIG = FALSE

GENSTART = IIIER

DO

IF TIMER-GONSTART > .5 THEII

PRIIT " WAITIMG FOR GEITEC TO ASSERT SRQ - IBSTA\% IS "; HEXS(IBSTA\%)

CALL IBRSP (G\%,SPRY)

GOSUB HaitGPIB

PRINT "SERTAL POLL RESPOUSE IS "; HEXS(SPRY)

if spry then

GenHUIG - TRUE

gosub ClearGentecSPR

GEnRESPOUSE = SPACES (256)

call IBND(G\%,GonRESPONSE\$)

end if

GenSTART = TIKER

END IF

CALL IBRD(G\%, MULS)

GOSUB NaitGPIB

LOOP UITIL (IBSTAY AID \&H0800)

call ibrup (gh, spr $\%)$

IF SPR\% <> th40 THEI PRIIT "GEITEC SPR\% - ";HEX\$(SPRZ) : GOSUB PRESSKEY GOSUB ClearGentecSPR 
- Clear any previous response and store the ner one

GenRESPOAsEsempaces (30)

CALL IBRD (G\%,GenRESPOUSES)

GOSUB HaitGPIB

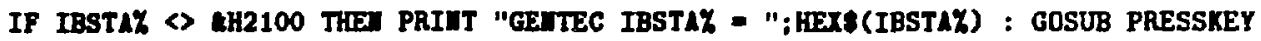

RETUR

ClearGentecSPR:

DO

- Try ThRE tines to reset the earial poll respone byte

CALL IBRSP(G\%, SPR\%)

CAI. IBRSP (G\%, SPR\%)

CAIL IBRSP(G\%, SPRY)

IF SPRK THEX PRIIT "SERIAL POLL HAS IOT RESET GEUTEC RESPOISE BYTE"

LOOP UHILE SPR\%

RETUR

ReleascGentec:

This returns the Gentec to local control.

' Othervise, the panel buttons remain locked out until the nert porer up.

CAL IBLOC(O\%)

RETURI

OOPS:

SELECT CASE ERR

CASE 53

'file not found

MoSuchFile=THE

RESUR TEXT

CASE 64

PRIII FILALi:; " is an invalid file neve"

IIPUT "Enter replaceent file nae including path ", FILسMs

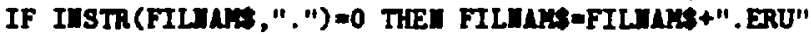

RESUI:

CASE 76

PRIII "Path to "; FILIArt; " was not found"

IIPUT "Enter replaceent file zane including path ", FILAKs

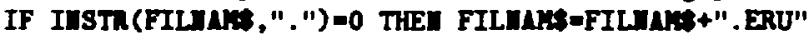

RESU,R

CISE EISE

OI ERROR COTO 0

EID SELECT 
5. DRUN

This variation of ERUN reads the status of a delay generator and stores spectra collected with the discharge on separately from those collected with the discharge off. Each set is still divided into energy bins. Subroutines which are the same as in ERUN are omitted from the listing.

- Read TR8818 digitizer, raed Gentec, ansig each data set to a bin

- according to its orn laser pulse energs

- Call DISCRIH instead of COIVERT to discard lov-voltage points phich

- naj be K.CP noive

MOTE: SUBROUTIUES WHICH ARE IDEITICAL TO THOSE IN ERUII ARE ORITTED

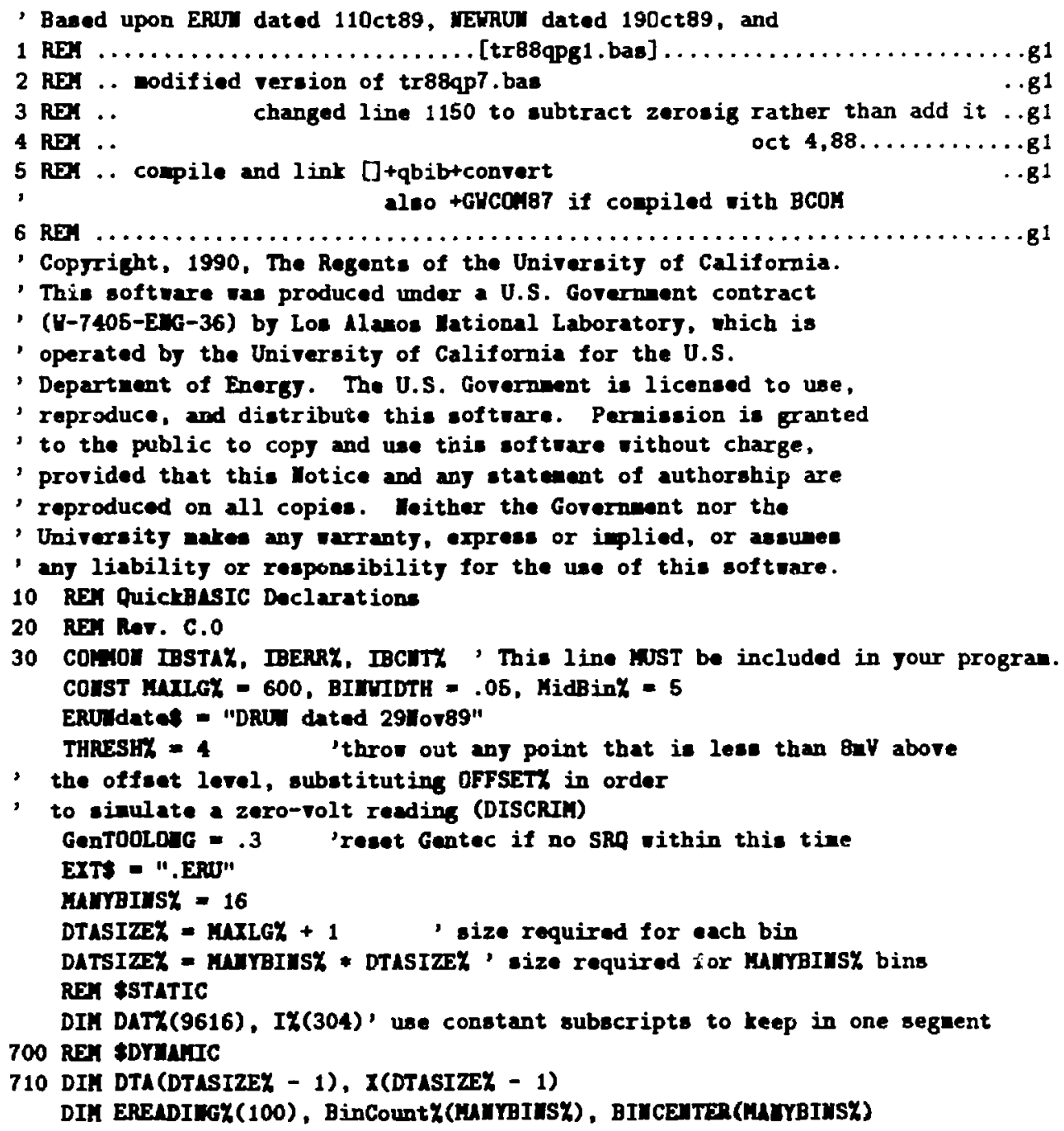




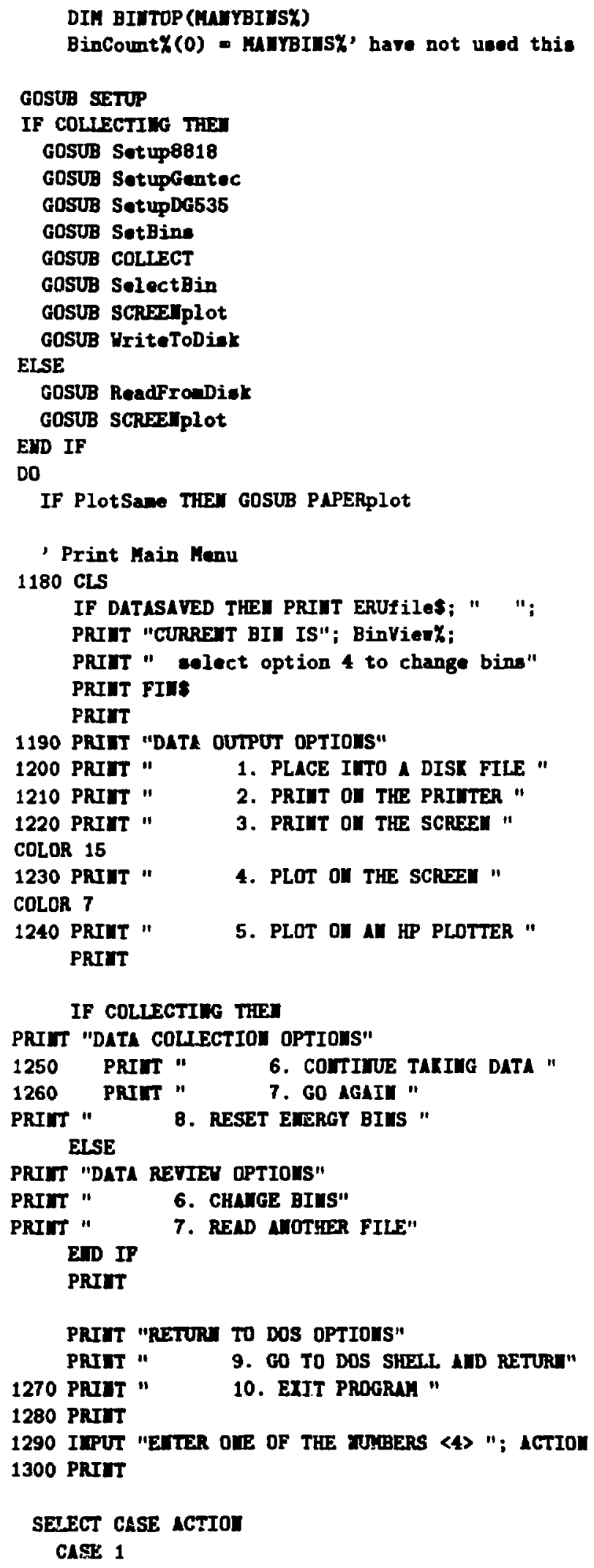




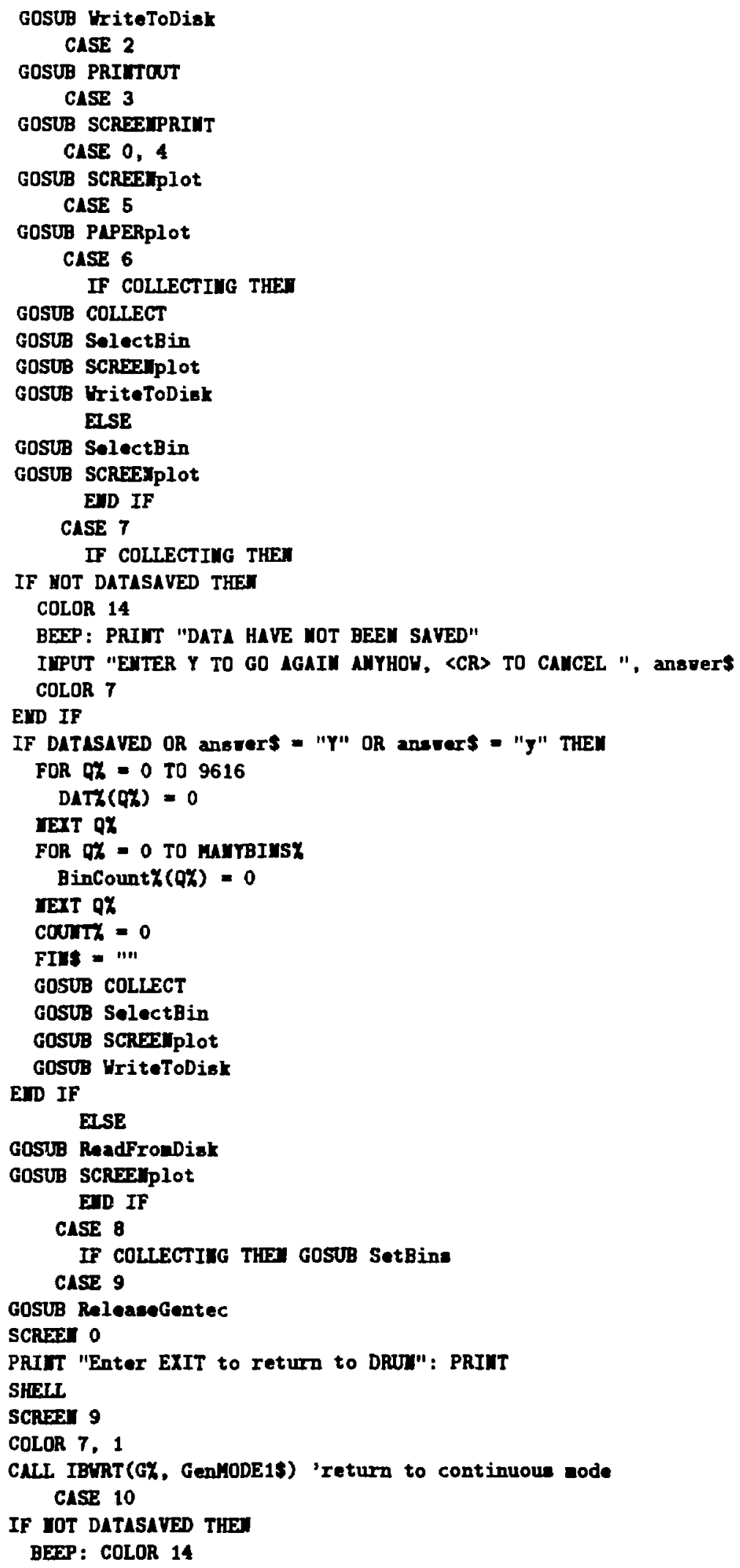




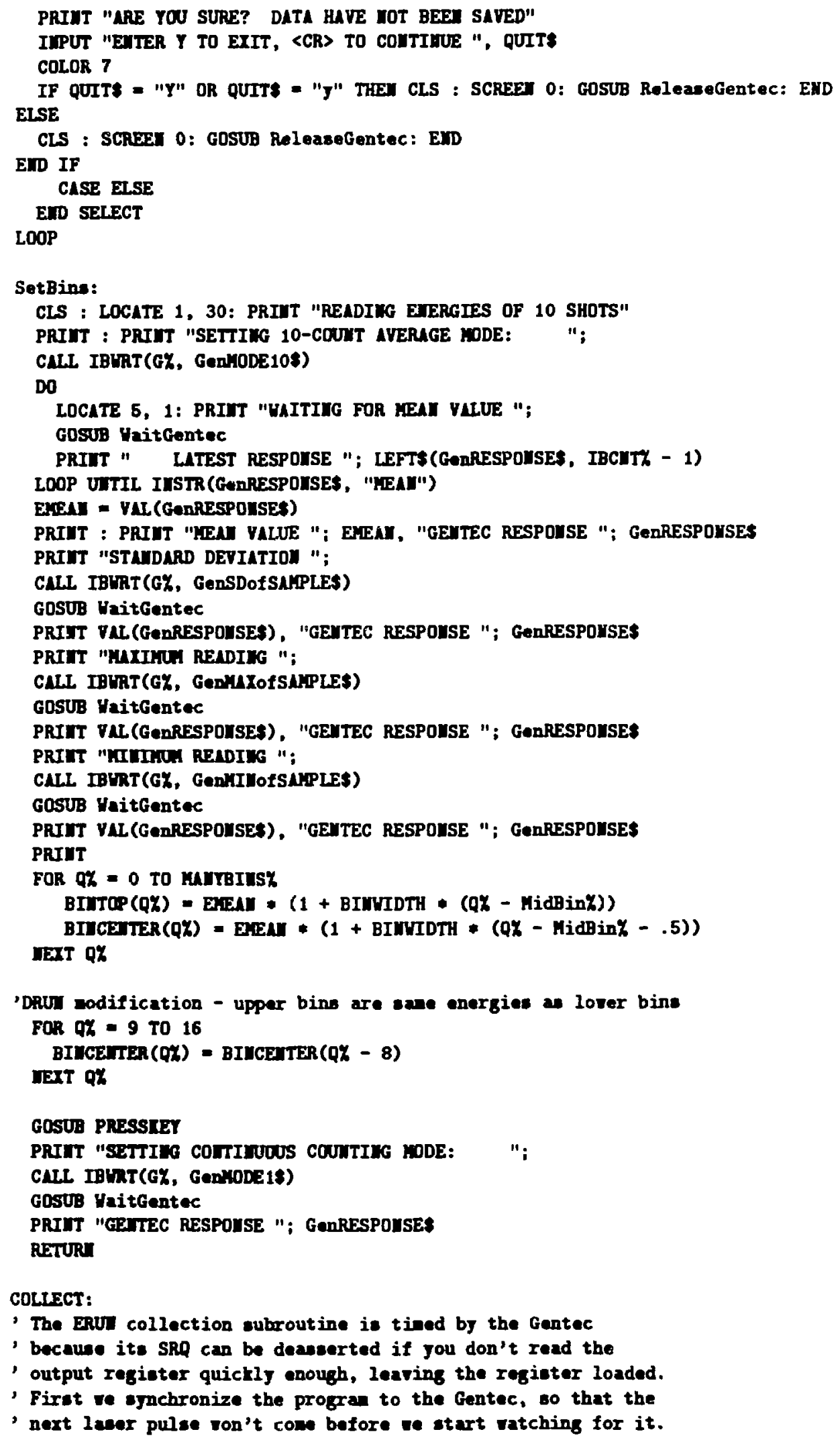


' We check that both derices are ready before each pulee,

' then otart the TR8818, then vait for the Gentec to detect

and neasure a laser pulse. By the tine an energy reading is

ready, the TR8818 should also be ready to read, but ve check

for its internal $\mathrm{LAN}$ in cane the photodiode trigger is

abent. When both derices have measured the sane laser

' shot, ve add the TR8818 data to the appropriate bin.

CLS

IF COUIT $=0$ THE

PRIIT "ENTER FILIER DEISITY <"; FilterDensity;

IMPUT "> ", answers

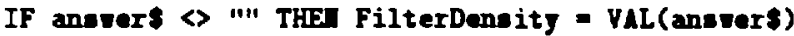

END IF

870 PRIIT "STRIKE AYY IEY TO EXIT DATA TAKIMg AT AIY TIKE", "COUNT NOH ="; COUNT"\%

880 IYPUT "ENIER ITRBER OF SHOTS OR ZERO FOR COITIWUOUS DATA TAKIYG"; SCOUNT\%

890 PRIMT : PRINT " DATA ACQUTSITION HOU TAKING PLACE."

'LOCATE 8, 35: PRIIT "COUIT", "BII"

VIEU $(10,80)-(629,250)$

UIMDOU (COUIT, -1$)-($ SCOUITh, 18)

LIIE (COUTTh, .5)-(SCOUITK, 16.5), B

LIIE (COUIT/, 8.5)-(SCOUIT/, 8.5), 8

LOCATE 18, 1: PRIIT COUITY;

LOCATE 18, 75: PRIIT SCOUIT\%;

IF COUMTY $=0$ THE STARTDATE\$ = DATE\$: STARTTIME\$ - TIHE\$

DATASAYED - FALSE

GOAGAII - FALSE

, Synchronize ERU: vith Gentec

CALI IBASP (GX, SPRX) : GOSUB WaitGPIB

CALL IBRD (GX, GenRESPOISES)'allov timeout in case output buffer is enpty

GOSUB ClearGentecSPR

GOSUB VaitGentec

CALL IBURT (DG\%, DGInntStatus\$): GOSUB WaitGPIB

CALI IBRD (DG\%, DGRasponse\$): GOSUB WaitGPIB

DO 'this starts the COUnT loop

DO

CALI. IBRSP(D1\%, SPRY): GOSUB UaitGPIB 'serial poll

" print "Initial 8818 spr $=$ "; hers (spr

LOOP WHILE (IBSTAY AKD \&H800) OR (SPRY \& 3)

DO

- Start TR8818

CALL IBURT(D1\%, IRF9\$): GOSUB WaitGPIB

CALL IBRD(D1\%, UUL\$): GOSUB UaitGPIB 'otart TR8818

CALL IBLRT(D1\%, TRF27\$)' Store next comand

, Clear Gentec output buffer, SRQ, and SPR

CALI IBRSP (GX, SPRY): GOSUB VaitGP IB

CALL IBRD(GY, GenRESPOISES)'allow tineout in case output buffer is empty

CALL IBRSP(GX, SPRY): GOSUB WaitGPIB

, GOSUB ClearGentecSPR

GOSUB WaitGentec

'test for trigger received by TR8818

CALL IBRSP (D1\%, SPRY)

InternalLAY $=($ SPEY $=3)$ 


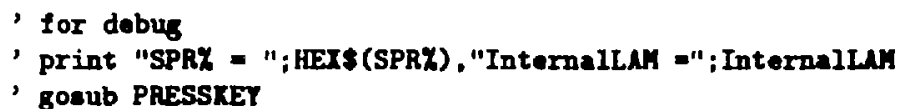
LOOP WHILE GonkUU, OR WOT Internallek

\section{'Read TR8818 data}

CALL IBLRT (D1X, TRF17\$) 'enable readout

CALL IBRSP(D1\%, SPRX): GOSUB YaitGPIB 'serial poll

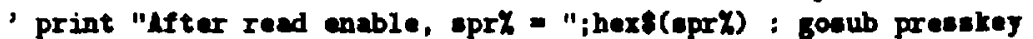

CALL IBURT(D1X, MES16blockread\$) : GOSUB YaitGPIB 'bigh epeed 16 bit block read

CALL IBURT(D1\%, TRF28): GOSUB UaitGPIB

CALL IBRDI(D1\%, IK(0), LGK + 4): GOSUB HaitGPIB'read data to IK array

- CALC IBRSP(D1\%,SPR\%) : GOSUB VaitGPIB 'sorial poll

, print "lfter block read, spr\% = ";her\$(spr $\%$ : gosub presskey

'Read DG535 Status

'CALL IBLRT (DG\%, DGInstStatueBit13): COSUB YaitGPIB

CALL IBURT (DCY, DGInEtStatus\$): GOSUB YaitGPIB

CALL IBRD(DGK, DGResponses): GOSUB UaitGPIB

DGStatus\% = VAL(DGResponses)

- find bin number - range is 1 to HArYBIus\%

- discard data falling above highest bin or belov lonest

ETERGY = VAL(GenRESPOUSES)

bin

IFOR Q\% - 0 TO HAIrEIMS

FOR of $=0$ TO 8

IF ETERGY < BIUTCP(QK) THEM ebinK = QK: EXIT FOR

IETT ox

LOCATE 21, 1: PRIIT " ";

IF DGStatus\% $=18$ THEX

'do nothing. Dait for $H$ atoes to be punped out

SOUID 40,1

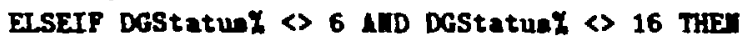

LOCATE 21, 1

PRIIT "* URECCGIIZED DG535 STATUS"; DGStatus\%; "(decinal) "

ELSEIF ebin\% > 0 AND bin\% < 9 THE

- For DaU, add 8 to bin number if DG535 is busy turning the

' discharge on (status bits 1 and 2 eet)

' If discharge is off, status bit 4 vill be left fron the last pulse

' to be ippored, and all othere vill be off

IF DGStatus $=6$ THE abin $\%=b$ bin $\%+8:$ DGcolor $\%$ - 13 ELSE DGcolor $\%=15$

'CALL DBUT(DGX, DGErrorStatus): GOSUB UaitGPIB this took too long

'CALL IBRD(DOX, DGResponse\$): GOSUB YajtGPIB

'IF VAL(DGResponses) THED PRITT "DG reported error "; DGResponses

BIHOFFSETK - (tbin -1$) *$ (DTASIZEK)

BinCount $\%$ (obin $\chi)=$ BinCount $\chi(a b i n \chi)+1$

$1050 x$ - PEEX (VARPTR(IK(0))) AND 1

1060 ADX $-\operatorname{VAPTR}(I \%(0))+2+x$

830 ODEK $=$ VLRPTR(DATK (BIMOFFSETK))

1070 coUTT $=$ CovmTh +1

'1080 LDCATE 10, 35: PRIIT COUNT\%, EBII\%

PSET (COUIIt, obin $\%$ ), DGcolor $\%$

1090 CAL DISCRIM(ADRK, ODRK, LGK + 1, OFFSETY, THRESHX) 
ELSE

' COUIY = COUIT + 1 'count only shots that are used

'color 14

'LOCATE 10, 35: PRIIT COUIT\%, EBIMY

'color 7

LIIE (COUTT, ebin $\%)-(\operatorname{cov}$ TT $\%, e b i n \%+.5), 14$

END IF

LOOP UITIL (COUTT = SCOUTTh) OR (IMKEYs «" "")

STOPTIME\$ - TIME:

VIEU' reset viewport

RETUR

SetupDG535:

'The Stanford Research DG535 delaj/pulee generator has default GPIB

'addrese of 15, which is naned DG535 in GPIB.COM

DGErrorStatuss = "ES" 'returns error status byte

DGInstStatus = "IS" 'returns instrument status bjte

DGInstStatusBit1s = "IS1" "returns bit 1 of instrunent status bjte

DGResponees = SPICEs(4) 'roon for tro hex digite, CR, and LF

CALL IBFIMD("DG535", DG\%): GOSUB YaitGPIB

CALL IBURT(DG\%, DGErrorStatus): GOSUB YaitGPIB

CALL. IBRD(DG\%, DGResponses): GOSUB vaitGPIB

IF VAL(DGResponses) THEI PRIIT "DG reported error "; DGResponses RETUR:

Releasefentec:

- This returns the Gentec and the DG535 to local control.

' Othervise, the panel buttons renain locked out until the next pover up.

CALC IBLDC(GY)

CALI IBLDC (DGL)

RETUR

6. DISCRIM

This variation of CONVERT 1 simulates a discriminator.

coment I

page . 132

nave DISCRIY
: 132 colum, default number of lines

; sets nodule nume

T. David Nichols 28110789

Subroutine to convert digitizer data array to BLSIC integer array etoring only those values that exceed a threshold

Copyright, 1990, The Regents of the University of California.

This softuare wa produced under a U.S. Goreranont contract

(U-7405-EUG-36) by Los Alasos Mational Laboratory, which is operated by the University of Californis for the U.S.

Departeent of Energy. The U.S. Gorernment is licensed to use, reproduce, and distribute this softeare. Pernission is granted to the public to copy and vae this softrare vithout charge, provided that this Motice and any statenent of authorship are reproduced on all copies. Weither the Governnent nor the University nakes any varranty, express or inplied, or ansures any liability or responsibility for the use of this softrare. 
This is sinilar to the COIVERT subroutine used sith QuickBasic 3.0 in TR8BQP7 and related prograns. Both arraye nust be located in DGROUP. Within the $\$ 84.5$ environent, this requires they be first dinensioned oith constant dimensions, then aseigned to colido. In 484.5 EXE files, thes need not be in cosion.

Calling sequence from QuickBasic 3.0 or 4.6

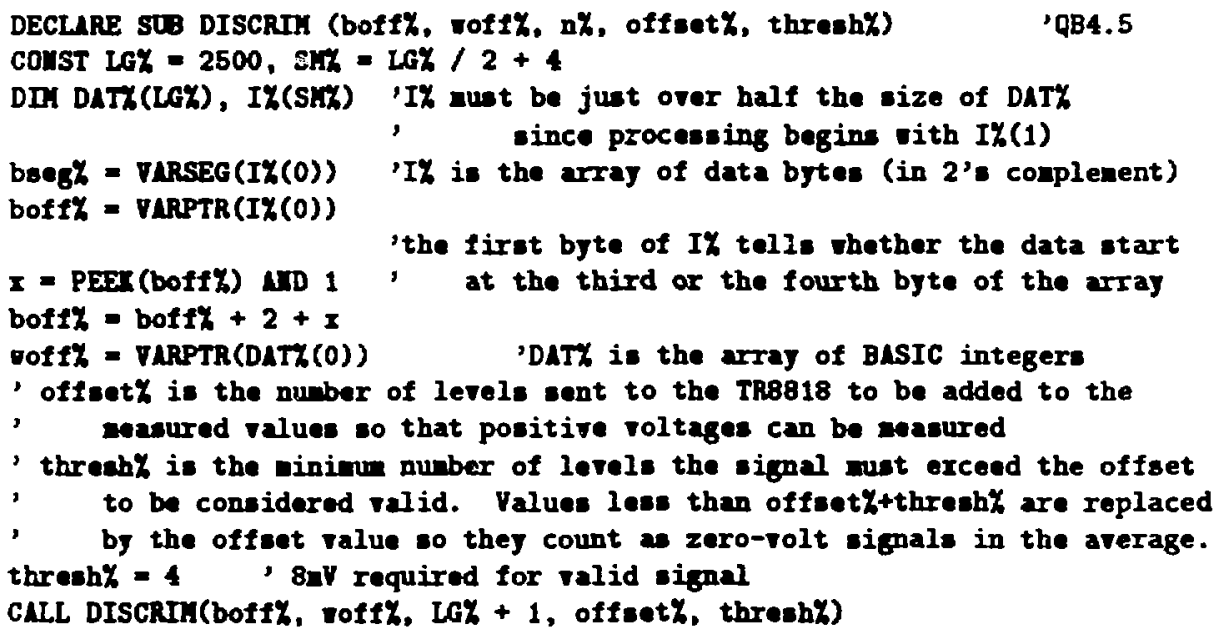

\section{MUSH DISCRIM;}

BUILDLIB warlib.obj prefix.obj DISCRIh.obj, xlib.obj (or naybe rlib.exe:) QB jones.bes /L xlib.ere

after cospiling with BCOH to generate jones.obj,

IIIIX jones+other+DISCRDI+GICON87

\section{Aasembling vith MSH 4.0 for use vith quickBasic $\mathbf{4 . 5}$}

MASH DISCRIM;

LIMX /Q DISCRIn.obj other.obj thaterer.obj, dislib.qLb, , IQB45 LBQLB45.LIB;

LIB dislib.lib +DISCRII.obj +other.obj + whatever.obj;

QB minprog.bas $/ 1$ dislib

This creates a Quick Library and a stand-alone library, each containing object code of DISCRIK, other, and vhaterer. Object files aay be added to or deleted from these files by LIB and by 18 iteelf (instruction in Learning and Uaing voluee of the QB4.5 manual). ".obj" may be onitted. File nawes in lover case represent actual nawe of files. The too library file nut have the sare base nare.

i print this title on esch page of printout title DISCRII

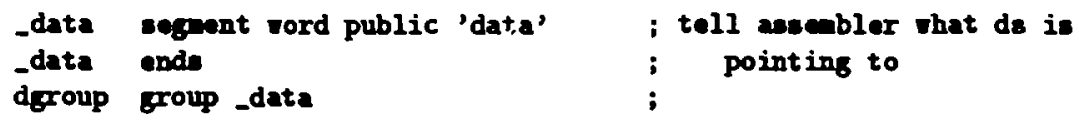

DISCRIH_text segent vord public 'code' ; start of code segaent 


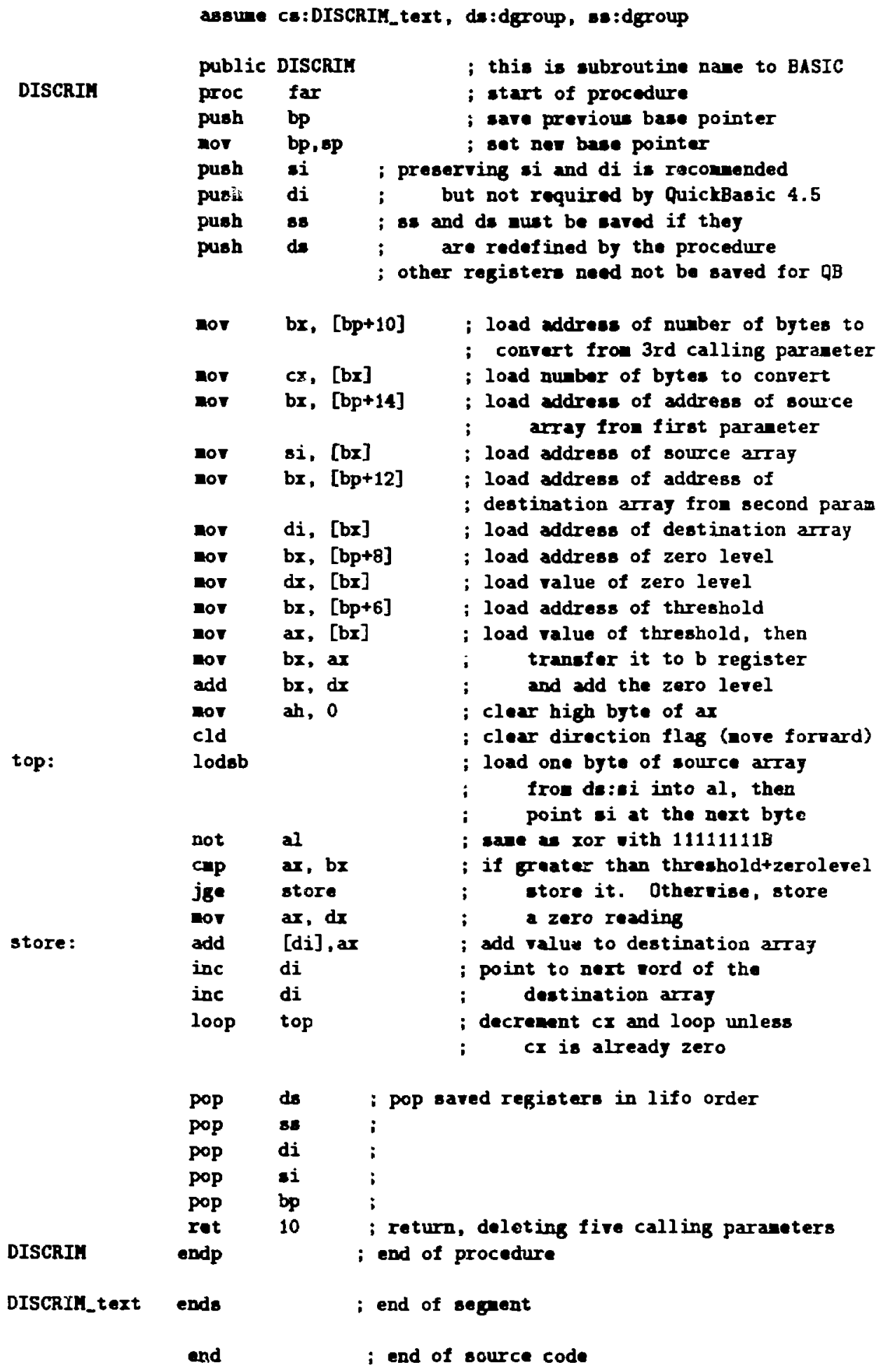




\section{REFERENCES}

P. Agostini, F. Fabre, G. Mainfray, G. Petite, and N. K. Rahman, "Free-free transitions following six-photon ionization of xenon atoms," Phys. Rev. Lett. 42, 1127-1130, (1979).

P. Agostini and G. Petite, "Photoelectric effect under strong irradiation," Contemp. Phys. 29, 57-77, (1988).

M. Aymar and M. Crance, "Multiphoton ionisation probabilities of multiple continua in alkali atoms," J. Phys. B 14, 3585-3601, (1981).

K. G. H. Baldwin and B. W. Boreham, "Investigation of tunneling processes in laser-induced ionization of argon," J. Appl. Phys. 52, 2627-2633, (1981).

W. Becker, R. R. Schlicher, and M. O. Scully, "Final-state effects in above-threshold ionisation," J. Phys. B 19, L785-L792, (1986).

W. Becker, R. R. Schlicher, M. O. Scully, and K. Wódkiewicz, "The role of final state effects in above-threshold ionization,"

J. Opt. Soc. Am. B 4, 743-751, (1987).

H. A. Bethe and E. E. Salpeter,

Quantum Mechanics of One- and Two-Electron Atoms (Springer, Berlin, 1957), p. 3.

Z. Białynicka-Birula and I. Białynicki-Birula, "Collective excitations of electrons by an intense wave in a magnetic field," J. Opt. Soc. Am. B 3, 1311-1314, (1986).

B. W. Boreham and H. Hora,

"Debye-length discrimination of nonlinear laser forces acting on electrons in tenuous plasmas,"

Phys. Rev. Lett. 42, 776-779, (1979).

M. Born and E. Wolf, Principles of Optics, 6th edition, (Pergamon Press, New York, 1980), p. 472.

D. Brenner,

"Regulation of atomic hydrogen flow with a platinum wire detector," Rev. Sci. Instrum. 40, 1234-1235, (1969). 


\section{R. Brewer,}

"Resonant multiphoton ionization of atomic hydrogen," Ph.D. thesis, Massischusetts Institute of Technology, 1984.

L. S. Brown and T. W. B. Kibble, "Interaction of Intense Laser Beams with Electrons," Phys. Rev. 133, A705-A719, (1964).

P. H. Bucksbaum, M. Bashkansky, R. R. Freeman, and T. J. McIlrath, "Suppression of multiphoton ionization with circularly polarized coherent light," Phys. Rev. Lett. 56, 2590-2593, (1986).

P. H. Bucksbaum, R. R. Freeman, M. Bashkansky, and T. J. McIlrath, "Role of the ponderomotive potential in above-threshold ionization," J. Opt. Soc. Am. B 4, 760-764, (1987).

M. R. Cervenan and N. R. Isenor, "Multiphoton ionization yield curves for Gaussian laser beams,"

Opt. Commun. 13, 175-178, (1975).

N. Chan, D. M. Crowe, M. S. Lubell, F. C. Tang, A. Vasilakis, F. J. Mulligan, and J. Slevin,

"A polarized atomic hydrogen beam,"

Z. Phys. D 10, 393-415, (1988).

S.-I Chu and W. P. Reinhardt,

"Intense field multiphoton ionization via complex dressed states: application to the $\mathrm{H}$ atom,"

Phys. Rev. Lett. 39, 1195-1198, (1977).

S.-I Chu and J. Cooper,

"Threshold shift and above-threshold multiphoton ionization of atomic hydrogen in intense laser fields,"

Phys. Rev. A 32, 2769-2775, (1985).

S.-I Chu and R. Y. Yin,

"Classical and quantal nonperturbative treatments of multiphoton and abovethreshold ionization,"

J. Opt. Soc. Am. B 4, 720-725, (1987).

S.-I Chu, private communication, 1990.

L. A. Collins and A. L. Merts,

"Atoms in strong, oscillating electric fields: momentum-space solutions of the time-dependent, three-dimensional Schrödinger equation,"

J. Opt. Soc. Am. B 7, 647-658, (1990).

P. B. Corkum, N. H. Burnett, and F. Brunel,

"Above-threshold ionization in the long-wavelength limit,"

Phys. Rev. Lett. 62, 1259-1262, (1989). 
M. Crance,

"Multiphoton ionisation towards multiple continua: the role of space charge," J. Phys. B 19, L267-L271, (1986).

M. Crance,

"Multiphoton ionisation of hydrogen. A non-perturbative calculation of strongfield effects,"

J. Phys. B 21, 2697-2708, (1988).

Z. Deng and J. H. Eberly,

"Multiphoton absorption above ionization threshold by atoms in strong laser fields,"

J. Opt. Soc. Am. B 2, 486-493, (1985).

S. W. Downey and R. S. Hozack,

"Saturation of three-photon ionization of atomic hydrogen and deuterium at $243 \mathrm{~nm}, "$

Opt. Lett. 14, 15-17, (1989).

J. H. Eberly and J. Javanainen,

"Above-threshold ionization,"

Eur. J. Phys. 9, 265-275, (1988).

A. Einstein,

Phys. Z., January, 1909.

F. H. M. Faisal,

"Collision of electrons with laser photons in a background potential,"

J. Phys. B 6, L312-L315, (1973).

F. H. M. Faisal,

Multiphoton Processes

(Plenum, New York; 1987).

D. Feldmann, B. Wolff, M. Wemhoener, and K. H. Welge,

"Above-threshold-ionization' of atomic hydrogen: Angular distribution of photoelectrons,"

Z. Phys. D 6, 293-294, (1987).

G. W. Fraser,

"The electron detection efficiency of microchannel plates,"

Nucl. Instrum. \& Methods 206, 445-449, (1983).

R. R. Freeman, P. H. Bucksbaum, H. Milchberg, S. Darack, D. Schumacher, and M. E. Geusic,

"Above-threshold jonization with subpicosecond laser pulses,"

Phys. Rev. Lett. 59, 1092-1095, (1987). 
B. Gao and A. F. Starace,

"Variational calculation of multiphoton ionization processes for the $\mathrm{H}$ atom,"

Phys. Rev. Lett. 61, 404-407, (1988).

B. Gao and A. F. Starace,

"Variational principle for high-order perturbations with application to multiphoton processes for the $\mathrm{H}$ atom,"

Phys. Rev. A 39, 4550-4560, (1989).

B. Gao, C. Pan, C.-R. Liu, and A. F. Starace,

"Variational methods for high-order multiphoton processes,"

J. Opt. Soc. Am. B 7, 622-630, (1990).

M. Göppert-Mayer,

"Über Elementarakte mit zwei Quantensprüngen,"

Ann. Phys. 9, 273-294, (1931).

Y. Gontier and M. Trahin,

"Multiphoton Ionization of Atomic Hydrogen in the Ground State,"

Phys. Rev. 172, 83-87, (1968).

Y. Gontier and M. Trahin,

"Effect of an intense radiation field on the discrete and continuum spectra of atomic hydrogen,"

Phys. Rev. A 40, 1351-1362, (1989).

Y. Gontier, N. K. Rahman, and M. Trahin,

"Calculation of total cross section and angular distribution of threshold and above-threshold ionization of atomic hydrogen at the fourth harmonic of a $\mathrm{Nd}^{3+}$-glass laser,"

Phys. Rev. A 34, 1112-1116, (1986).

Y. Gontier, N. K. Rahman, and M. Trahin,

"Angular distribution of above-threshold ionization of atomic hydrogen.,"

Europhys. Lett. 5, 595-599, (1988).

D. R. Hartree,

"The wave mechanics of an atom with a non-Coulomb central field. Part I. Theory and methods,"

Proc. Cambridge Philos. Soc. 24, 89-132, (1928).

W. C. Henneberger,

"Perturbation method for atoms in intense light beams,"

Phys. Rev. Lett. 21, 838-841, (1968).

M. J. Hollis,

"Multiphoton ionization and EM field gradient forces,"

Opt. Commun. 25, 395-398, (1978). 
H. J. Humpert, H. Schwier, R. Hippler, and H. O. Lutz,

"Angular distribution of photoelectrons from above-threshold ionization of Xe," Phys. Rev. A 32, 3787-3789, (1985).

J. Javanainen and J. H. Eberly, "Model $a b$ initio study of intense laser photoionisation,"

J. Phys. B 21, L93-L98, (1988).

J. Javanainen, J. H. Eberly, and Q. Su,

"Numerical simulations of multiphoton ionization and above-threshold electron spectra,"

Phys. Rev. A 38, 3430-3446, (1988).

E. Karule,

Atomic processes,

(Latvian Academy of Sciences, Riga, 1975), p. 5, in Russian.

E. Karule,

"Multiphoton ionization of hydrogen by extra photons"

in Electronic and Atomic Collisions, edited by H. B. Gilbody, W. R. Newell,

F. H. Read, and A. C. H. Smith (Elsevier, Amsterdam, 1988), pp. 569-578.

E. Karule,

"Above-threshold ionization of atomic hydrogen,"

J. Opt. Soc. Am. B 7, 631-638, (1990).

L. V. Keldysh,

"Ionization in the field of a strong electromagnetic wave,"

Sov. Phys. JETP 20, 1307-1314, (1965).

translation of Zh. Eksp. Teor. Fiz. 47, 1945-1957 (1964).

D. E. Kelleher, M. Ligare, and L. R. Brewer,

"Resonant four-photon ionization of atomic hydrogen,"

Phys. Rev. A 31, 2747-2749, (1985).

S. V. Khristenko and S. I. Vetchinkin,

"Multiphoton ionization of a hydrogen atom,"

Opt. Spectrosc. 40, 239-241, (1976).

translation of Opt. Spektrosk. 40, 417-432 (1976).

T. W. B. Kibble,

"Mutual refraction of electrons and photons,"

Phys. Rev. 150, 1060-1069, (1966a).

T. W. B. Kibble,

"Refraction of electron beams by intense electromagnetic waves,"

Phys. Rev. Lett. 16, 1054-1056, (1966b). 
P. Kruit, J. Kimman, H. G. Muller, and M. J. van der Wiel, "Electron spectra from multiphoton ionization of xenon at 1064, 532, and $355 \mathrm{~nm}, "$

Phys. Rev. A 28, 248-255, (1983).

P. Kruit and F. H. Read,

"Magnetic field paralleliser for $2 \pi$ electron-spectrometer and electron-image magnifier,"

J. Phys. E 16, 313-324, (1983).

K. C. Kulander,

"Multiphoton ionization of hydrogen: A time-dependent theory,"

Phys. Rev. A 35, 445-447, (1987a).

K. C. Kulander,

"Time-dependent Hartree-Fock theory of multiphoton ionization: Helium,"

Phys. Rev. A 36, 2726-2738, (1 $087 b)$.

K. C. Kulander,

"Time-dependent theory of multiphoton ionization of xenon,"

Phys. Rev. A 38, 778-787, (1988).

G. A. Kyrala,

"Multiphoton ionization: a Monte Carlo treatment of a classical hydrogen atom in a classical electromagnetic field,"

J. Opt. Soc. Am. B 4, 731-738, (1987).

K. J. LaGattuta,

"Laser effects in photoionization: II. Numerical solutions of coupled equations for atomic hydrogen,"

Phys. Rev. A 41, 5110-5116, (1990a).

K. J. LaGattuta,

"Laser effects in photoionization: numerical solution of coupled equations for a three-dimensional Coulomb potential,"

J. Opt. Soc. Am. B 7, 639-646, (1990b).

P. Lambropoulos,

"Comments on major problems in multiphoton processes"

in Multiphoton processes, proceedings of the Fourth International Conference on Multiphoton Processes, Boulder, Colorado, 1987, edited by S. J. Smith and P. L. Knight (Cambridge University Press, Cambridge, 1988), pp. 350-356.

G. Laplanche, A. Durrieu, Y. Flank, M. Jaouen, and A. Rachman,

"The infinite summations of perturbation theory in atomic multiphoton ionization,"

J. Phys. B 9, 1263-1277, (1976). 
C. Leone, R. Burlon, F. Trombetta, S. Basile, and G. Ferrante,

"Strong-field multiphoton ionization of hydrogen. The S-Matrix treatment of the elementary process,"

Nuovo Cimento D 9, 609-630, (1987).

T. S. Luk, T. Graber, H. Jara, U. Johann, K. Boyer, and C. K. Rhodes, "Subpicosecond ultraviolet multiphoton electron spectroscopy of rare gases,"

J. Opt. Soc. Am. B 4, 847-851, (1987).

T. S. Luk and C. K. Rhodes,

"Multiphoton dissociative ionization of molecular deuterium,"

Phys. Rev. A 38, 6180-6184, (1988).

M. LuVan, G. Mainfray, C. Manus, and I. Tugov,

"Multiphoton ionization of atomic and molecular hydrogen at $0.53 \mu$,"

Phys. Rev. A 7, 91-98, (1973).

L. Marshall,

"Applications a la mode,"

Laser Focus , 26-28, (April 1971).

A. Maquet,

"Use of the Coulomb Green's function in atomic calculations,"

Phys. Rev. A 15, 1088-1108, (1977).

E. A. Martin and L. Mandel,

"Electron energy spectrum in laser-induced multiphoton ionization of atoms," Appl. Opt. 15, 2378-2380, (1976).

H. G. Muller, H. B. van Linden van den Heuvell, and M. J. van der Wiel, "Experiments on 'above-threshold ionization' of atomic hydrogen,"

Phys. Rev. A 34, 236-243, (1986).

L. Pan,

"The Volkov-like Coulomb continuum,"

Mod. Opt. 36, 877-889, (1989).

M. D. Perry, O. L. Landen, and A. Szöke,

"Measurement of the local laser intensity by photoelectron energy shifts in multiphoton ionization,"

J. Opt. Soc. Am. B 6, 344-349, (1989).

G. Petite, P. Agostini, and H. G. Muller,

"Intensity dependence of non-perturbative above-threshold ionisation spectra: experimental study,"

J. Phys. B 21, 4097-4105, (1988).

M. S. Pindzola and M. Dörr,

"Multiphoton ionization of hydrogen by an intense $248 \mathrm{~nm}$ linearly polarized field,"

Bull. A. P. S. 35, 1151, (1990). 
R. M. Potvliege and R. Shakeshaft, "High-order above-theshold ionization of hydrogen in perturbation theory," Phys. Rev. A 39, 1545-1548, (1989a).

R. M. Potvliege and R. Shakeshaft,

"Multipkoton processes in an intense laser field: Harmonic generation and total ionization rates for atomic hydrogen,"

Phys. Rev. A 40, 3061-3079, (1989b).

V. C. Reed and K. Burnett,

"Ionization of atoms in intense laser pulses using the Kramers-Henneberger transformation,"

Phys. Rev. A 42, 3152-3155, (1990).

H. R. Reiss,

"Effect of an intense electromagnetic field on a weakly bound system,"

Phys. Rev. A 22, 1786-1813, (1980).

H. R. Reiss,

"Electron spectrum in intense-field photoionization,"

J. Opt. Soc. Am. B 4, 726-730, (1987).

H. R. Reiss,

"Complete Keldysh theory and its limiting cases,"

Phys. Rev. A 42, 1476-1486, (1990).

J. P. Roberts, A. J. Taylor, P. H. Y. Lee, and R. B. Gibson,

"High-irradiance 248-nm laser system,"

Opt. Lett. 13, 734-736, (1988).

H. Rottke, B. Wolf, M. Brickwedde, D. Feldmann, and K. H. Welge,

"Multiphoton ionization of atomic hydrogen in intense subpicosecond laser pulses,"

Phys. Rev. Lett. 64, 404-407, (1990).

M. W. Sasnett,

"Propagation of multimode laser beams - The $M^{2}$ factor"

in The Physics and Technology of Laser Resonators, edited by D. R. Hall and P. E. Jackson (Adam Hilger, Bristol and New York, 1989), pp. 132-142.

R. Shakeshaft,

"Application of the Sturmian expansion to multiphoton absorption by hydrogen above the ionization threshold,"

Phys. Rev. A 34, 5119-5122, (1986).

R. Shakeshaft and R. M. Potvliege,

"Approximate theory for multiphoton ionization of an atom by an intense field," Phys. Rev. A 36, 5478-5481, (1987). 
J. H. Shirley,

"Solution of the Schrödinger equation with a Hamiltonian periodic in time,"

Phys. Rev. 138, B979-B987, (1965).

J. Slevin and W. Stirling,

"Radio frequency atomic hydrogen beam source,"

Rev. Sci. Instrum. 52, 1780-1782, (1981).

M. R. Sogard,

"Nonresonant multiphoton-ionization yields from Gaussian laser beams,"

J. Opt. Soc. Am. B 5, 1890-1899, (1988).

B. Sundaram and L. Armstrong, Jr.,

"Modeling strong-field above-threshold ionization,"

J. Opt. Soc. Am. B 7, 414-424, (1990).

V. Véniard and B. Piraux,

"Continuum-continuum dipole transitions in femtosecond-laser-pulse excitation of atomic hydrogen,"

Phys. Rev. A 41, 4019-4034, (1990).

J. W. Verschuur, L. D. Noordam, and H. B. van Linden van den Heuvell,

"Anomalies in above-threshold ionization observed in $\mathrm{H}_{2}$ and its excited fragments,"

Phys. Rev. A 40, 4383-4391, (1989).

D. M. Volkov,

"Über eine Klasse von Lösungen der Diracschen Gleichung,"

Z. Phys. 94, 250-260, (1935).

N. R. Walet,

"Lifetime of a hydrogen atom in an intense radiation field,"

Phys. Rev. A 41, 3905-3915, (1990).

R. C. Weast, editor

Handbook of Chemistry and Physics, 46th edition

(Chemical Rubber Company, Cleveland, 1965), pp. E61 and E143.

J. L. Wiza,

"Microchannel plate detectors,"

Nucl. Instrum. 162, 587-601, (1979).

B. Wolf, H. Rottke, D. Feldmann and K. H. Welge,

"Multiphoton-ionization of hydrogen atoms in intense laserfields,"

Z. Phys. D 10, 35-43, (1988).

W. Xiong, F. Yergeau, S. L. Chin, and P. Lavigne,

"Multiphoton ionisation of rare gases by a $\mathrm{CO}_{2}$ laser: electron spectroscopy,"

J. Phys. B 21, L159-L164, (1988). 
F. Yergeau, G. Petite, and P. Agostini,

"Above-threshold ionisation without space charge,"

J. Phys. B 19, L663-L669, (1986). 